\title{
Exploring antibody engineering technology for diagnostic applications
}

Citation for published version (APA):

de Haard, J. J. W. (1999). Exploring antibody engineering technology for diagnostic applications. [Doctoral Thesis, Maastricht University]. Universiteit Maastricht. https://doi.org/10.26481/dis.19990519jh

Document status and date:

Published: 01/01/1999

DOI:

10.26481/dis.19990519jh

Document Version:

Publisher's PDF, also known as Version of record

\section{Please check the document version of this publication:}

- A submitted manuscript is the version of the article upon submission and before peer-review. There can be important differences between the submitted version and the official published version of record.

People interested in the research are advised to contact the author for the final version of the publication, or visit the DOI to the publisher's website.

- The final author version and the galley proof are versions of the publication after peer review.

- The final published version features the final layout of the paper including the volume, issue and page numbers.

Link to publication

\footnotetext{
General rights rights.

- You may freely distribute the URL identifying the publication in the public portal. please follow below link for the End User Agreement:

www.umlib.nl/taverne-license

Take down policy

If you believe that this document breaches copyright please contact us at:

repository@maastrichtuniversity.nl

providing details and we will investigate your claim.
}

Copyright and moral rights for the publications made accessible in the public portal are retained by the authors and/or other copyright owners and it is a condition of accessing publications that users recognise and abide by the legal requirements associated with these

- Users may download and print one copy of any publication from the public portal for the purpose of private study or research.

- You may not further distribute the material or use it for any profit-making activity or commercial gain

If the publication is distributed under the terms of Article $25 \mathrm{fa}$ of the Dutch Copyright Act, indicated by the "Taverne" license above, 
Exploring Antibody Engineering Technology for Diagnostic Applications 
DE HAARD, Johannes Jozef Wilhelmus

Exploring Antibody Engineering Technology for Diagnostic Applications / Johannes Jozef Wilhelmus DE HAARD

Thesis Maastricht University, Maastricht. -With ref.-

With summary in Dutch

ISBN 90-6464-802-6

The research described in this thesis was performed at the Biosciences Research Unit of Organon Teknika, Boxtel, The Netherlands, and at CESAME at the dept. of Pathology, Maastricht University Hospital, Maastricht, The Netherlands. This work was supported by PBTS grant BIO 91028 from the Dutch Ministry of Economic Affairs. Financial support for printing this thesis was provided by Organon Teknika N.V., Boxtel, and Target Quest B.V., Maastricht. 


\title{
Exploring Antibody Engineering Technology for Diagnostic Applications
}

\author{
Proefschrift
}

ter verkrijging van de graad van doctor aan de Universiteit Maastricht

op gezag van de Rector Magnificus, Prof. dr. A.C. Nieuwenhuijzen Kruseman, volgens het besluit van het College van Decanen, in het openbaar te verdedigen op woensdag 19 mei 1999 om 16.00 uur

door

Johannes Jozef Wilhelmus de Haard

geboren te Beek bij Ubbergen op 11 juli 1959 


\section{Promotor :}

Prof. Dr. J.W. Arends

\section{Co-promotores :}

Dr. ir. H.R.J.M. Hoogenboom

Dr. B. van Gemen (Organon Teknika, Boxtel)

\section{Beoordelingscommissie :}

Prof. dr. J. de Haan (voorzitter)

Prof. dr. P.J.C.van Breda Vriesman

Prof. dr. M.P. van Dieijen-Visser

Dr. A.D. Griftiths (Centre for Protein Engineering, MRC, Cambridge, UK)

Prof. dr. P.J. Hudson (CRC for Diagnostic Technologies, CSIRO, Melbourne, Australia) 
Aan Willeke, Monique, Saskia en Eline 



\section{Contents}

INTRODUCTION

1 Antibodies in in vitro diagnostics

2 Phage display technology

2.1 Expression of antibody fragments in $E$. coli

2.2 Display of antibody fragments

2.3 Library technology

2.4 Selection strategies

2.5 Screening assays

3 Antibody repertoire cloning

3.1 Immune repertoires $\quad 22$

3.2 Naive repertoires 24

3.3 Synthetic repertoires 25

3.4 Making large repertoires 27

4 Perspectives $\quad 29$

4.1 Perspectives on selection technologies 29

4.2 Perspectives on antibody engineering $\quad 30$

5 Aim of the study $\quad 30$

References $\quad 32$

Chapter 1. EfFect OF a FRl mutation on Folding OF VH

Chapter 2. A FRI MUTATION IN VL AFFECTING ANTIGEN BINDING

ChaPter 3. QUANTIFICATION OF SINGLE CHAIN FV'S WITH SURFACE PLASMON RESONANCE

Chapter 4. Single CHAIN FV'S AGAinst HCG From A MURINE IMMUNE LIBRARY

Chapter 5. SElection Of MURINE ANTI- HIV-1 P24 ANTIBOdy FRAGMENTS

Chapter 6. Selection of human anti-HIV-1 ENVElope SINGle Chain ANTIBODIES 
ChaPTER 7. ANTI- GLYCOPROTEIN HORMONE FAB'S FROM A NAÏVE HUMAN REPERTOIRE

SUMMARY; SAMENVATTING

1 Exploring Antibody Engineering Technology for Diagnostic Applications; summary

1.1 Library derived anti-hCG scFv's

1.2 Anti-HIV-1 scFv's

1.3 Construction of and selections from a naiive human repertoire

2 Samenvatting

200

2.1 Anti-hCG scFv-s geïsoleerd uit een fiag bank

2.2 Anti-HIV-I scFv's

2.3 De konstruktie van en selekties uit een naïeve humane bank

DANKWOORD 


\section{INTRODUCTION}

Adapted from

Creating and engineering human antibodies for immunotherapy

Hans J.W. de Haard, Paula Henderikx, Hennie R.J.M. Hoogenboom Advanced Drug Delivery Reviews

1998; Volume 31, Nos. 1,2; pages 5 - 31 


\section{Antibodies in in vitro diagnostics}

In vitro diagnostics find their application in the diflerent disciplines of science, ranging from virulogy, where they enabled the identification of infected sera. to endocrinology, for the detution of hormones, or in the field of haemostasis. The immuno-assay plays also an important role in fundamental research, where it aids in the characterisation of proteins or other antigens, and certainly it will be applied in the coming era of Genomics for the characterisation of new discovered gene products. Traditionally, antibodies form the basic ingredients of diagnostic products. After the introduction of hybridoma technology [1], when homogeneous batches of monoclonal antibodies became available, the sensitivity and the specificity of the assiays improved significantly as the result from the predefined antigen recognition. Substitution of (one of the) polyclonal by monoclonal antibodies for capture or detection reduced background problems. while the use of high affinity antibodies increased the sensitivity of the assays. By their specific epitope recognition, it was feasible to design tests which only detected the desired antigen, and not closely related products.

Genctic engineering of antibodies has already been applied in the field of diagnostics, for instince a commercially available, whole-blood agglutination assay was made with a scFv. directed against the surface antigen glycophorin A of red blood cells. fused to human inmmunodeficiency virus (HIV) gp36 or gp46 peptides [2,3]. Genetically linkage of the antigen binding domain with desired effector functions might also be useful for immuno-assays: Laukkanen and colleagues expressed immuno-liposomes [4], and in vivo produced alkaline phosphatase antibody conjugates have been generated by several groups [5,6]. A new era may have started in the generation of diagnostic reagents by phage display of antibody fragments. This technology has allowed access to large collections of different phage antibodies, created by the cloning of antibody V-genes from B-cells. Antibodies against any type of antigen may be derived from such repertoires, by rounds of enrichment on antigen and re-amplification. Moreover, by the availability of the encoding antibody genes, the selected fragments can be engineered on affinity and the molecular format aiming at their application.

This thesis addresses in detail the engineering and selection of antibodies from display repertoires, aiming at their application in in vitro diagnostics. In paragraph 2 of the Introduction, the principles of the repertoire technology are described. These include the expression of antibody fragments in the prokaryotic host $E$. coli, the display on filamentous phage and, associated with this, the issue of the selection methodology, by which phage, that expose antigen specific antibody fragments, are isolated from repertoires. Subsequently the different kind of repertoires and their construction are presented in paragraph 3, while in paragraph 4 we speculate about the perspectives of the technology. Finally, in paragraph 5 the aim of the study will be outlined. 


\section{Phage display technology}

The use of phage to display antibody fragments hinges on the expression of functional antibody fragments in bacterial hosts, which will be reviewed first.

\subsection{Expression of antibody fragments in E. coli}

Correctly folded antibody fragments. Fab. Fv or single-chain Fv (scFv) fragments, are expressed in $E$. coli by routing the nascent antibody chain(s) to the periplasm of the bacterium [7,8] (reviewed in [9]), where the intradomain disulphide bridge between the two $\beta$-shects is formed and the $V_{H}$ and $V_{L}$ will pair. Periplasmic expression in the bacterium resembles the natural production route in the endoplasmic reticulum (ER) of the lymphocyte. Both the heavy and light immunoglobulin chains carry a signal peptide, which is cleaved during transport from the cyloplasm over the membrane into the lumen of the ER, where the disulphide bridges are formed. Direct cytoplasmic expression does not yield functional antibody fragments, unless the molecule is refolded in vitro, for example from inclusion bodies [10,11].

Many different antibody formats have been expressed in E. coli, including Fith [8,12], Fv [7], $s c F v[11,13,14]$, antibodies with one V-gene domain only [15], and fragments thereof [16]. In the $s c F v$, the most popular format, the two variable domains are fused via a flexible linker region, and many hybrid formats. A linker was used to stabilise the FV-fragment, containing the $\mathrm{V}_{\mathrm{H}^{-}}$and $\mathrm{V}_{\mathrm{L}}$-molecules, because, although spontaneous assembly of $\mathrm{V}_{\mathrm{H}}$ and $\mathrm{V}_{\mathrm{L}}$ domains occurs in the periplasm of the bacteria, they tend to dissociate upon dilution [14]. The order of the Vdomains may be varied, with the $\mathrm{V}_{\mathrm{H}}$-domain at the amino terminus [13] or at the carboxy terminus [11], albeit with adjustment of the linker length for optimal spatial arrangement of the two $\mathrm{V}$-domains $[17,18]$. The most frequently used format, $\mathrm{V}_{\mathrm{H}}$-(Gly4Ser $)_{3}-\mathrm{V}_{\mathrm{L}}$ has been used extensively for the construction of phage libraries [19,20.21] (see also further). In a few cases, it has been reported that the $\mathrm{V}_{\mathrm{H}}$-linker $-\mathrm{V}_{\mathrm{L}}$ format did not yield a functional scFv, due to involvement of the $\mathrm{N}$ - or $\mathrm{C}$-terminal regions of $\mathrm{V}_{\mathrm{H}}$ and $\mathrm{V}_{\mathrm{L}}$ in antigen binding; for example, the $\mathrm{N}$-terminal $\mathrm{V}_{\mathrm{H}}$ region may be oriented closely to the CDR3 of the $\mathrm{V}_{\mathrm{H}}$, and its fusion to a linker sequence may affect antigen binding [22]. In one instance, positive charges in the amino terminal part of the $\mathrm{V}_{\mathrm{H}}$-region were implicated in blocking scFv transpurt across the bacterial membrane [23]. It should be kept in mind that these factors, apart from technical reasons, may explain the occasional difficulties found in trying to express functional scFv fragments $[24,25,26]$.

Alternatively, Fv may be stabilised by the introduction of a disulphide bridge between the variable light and heavy chain domains [34,27]; when introduced in a scFv, the thermal stability is enhanced also [28]. For some antibodies, such Fv-variants may have better anti-tumor activities than the scFv analogue, for example, when injected as immunotoxin $[29,30]$.

Conversion from the hybridoma-produced antibody into a bacterially expressed fragment might be needed for modelling and humanization [31], for crystallization [32,33,34] or for 
analysis of residues in the antibody involved in antigen binding by combining mutagenesis and $\mathrm{X}$-ray diffraction [35]. Since numerous researchers encounter problems when cloning antibody V-genes from monoclonal antibodies for expression in bacterial hosts, it is worthwhile spending some time on its causes. Hybridoma V-genes may be isolated by application of the polymerase chain reaction (PCR) from the B-cell cDNA [36,37,38,39]. Several primer sets for the amplification of murine and rat $\mathrm{V}$-genes have been described (details are given below). The $\mathrm{V}$ genes are then cloned into an appropriate expression vector [40]. Many of the cloning problems are related to not finding the correct, antigen binding $V$-gene pair $[24,25,26,41,42]$. This is often caused by the transcription of high amounts of aberrant heavy and light chain mRNAs in the hybridoma, derived from unsuccessful V-gene recombinations in the B-cell or myeloma fusion partner. or the hybridoma is a fusion product of different B-cells. Frequently therefore, a high frequency of non-antigen binding antibody fragments may be found after cloning. Furthermore, the correct V-genes may have acquired mutations associated with the PCR method, or may have lost (part of) the antigen binding due to oligonucleotide-induced changes of the $\mathrm{N}$ - or C-terminal region, which unpredictably affect antigen binding (see chapter 1 and 2 of this thesis).

The availability of PCR primers for the generation of large repertoires of $V$-genes led to the idea of accessing not just one but thousands or millions of different antibody fragments expressed in E. coli. From a repertoire of $\mathrm{V}_{\mathrm{H}}$-domains derived from mice immunized with lysozyme, Ward et al. [40] isolated anti-lysozyme single domain antibodies, by filter screening the bacteria. Alternatively, phage lambda was used, in combination with plaque screening [37]. Both approaches were restricted to the screening of a limited number of clones, and on the availability of large amounts of labeled antigen. Although they provided the concepts for creating and accessing large antibody repertoires, the real potential of combinatorial repertoires was established using phage antibody display.

\subsection{Display of antibody fragments}

Antibodies were the first proteins to be displayed successfully on the surface of phage [43]. This was achieved by fusing the coding sequence of the antibody variable (V) regions encoding a single-chain Fv fragment to the amino terminus of the phage minor coat protein pIII. Expression of the fusion product and its subsequent incorporation into the mature phage coat results in the antibody being presented on the phage surface, while its genetic material resides within the phage particle. Phage display was originally described in 1985 by Smith [44], who illustrated the use of the filamentous bacteriophage td for the display of peptides on the phage coat (for review see [45]).

Non-lytic filamentous phage infect strains of $E$. coli that harbour the $\mathrm{F}$ conjugative plasmid. The phage particles attach to the tip of the $\mathrm{F}$ pilus, encoded by genes on this plasmid, and the phage genome, a circular single-stranded DNA molecule, is translocated into the cytoplasm. The genome is replicated involving both phage- and host-derived proteins, and packaged by the 
infected cell into a rod-shaped particle, which is released into the media. All virion proteins will undergo transport to the cell periplasm prior to assembly and extrusion. The particle is essentially the phage genome covered by thousands of copies of a small capsid protein ( $p$ VIII), and, al one end of the particle, by five copies of the adsorption protein, the product of gene III, which mediates binding to the F-pilus. For further details on phage biology, see ref. [46].

In 1990, the display of a single-chain antibody fragment on the surface of the filamentous phage was demonstrated, as well as the possibility of selecting the antibody displaying phage from a mixture of irrelevant phage [43]. The first antibody was displayed using a phage vector, based on phage fd-tet [47] and its gene III as fusion partner. In this vector. the genes encoding an antibody scFv fragment are cloned in frame with gene III and downstream of the genc III signal sequence, which normally directs the adsorption protein to the periplasm. Here, the $V_{H}$ and $V_{L}$ domains will fold correctly, stabilised by an intramolecular disulphide-bridge, and pair to form a functional scFv. A phage vector carries all of the genetic information required for the phage's life cycle. Phagemids, a more popular vector for display, are plasmid vectors that carry gene III with appropriate cloning sites, and a phage packaging signal (Fig. 1) [48,49,50]. The phagemid

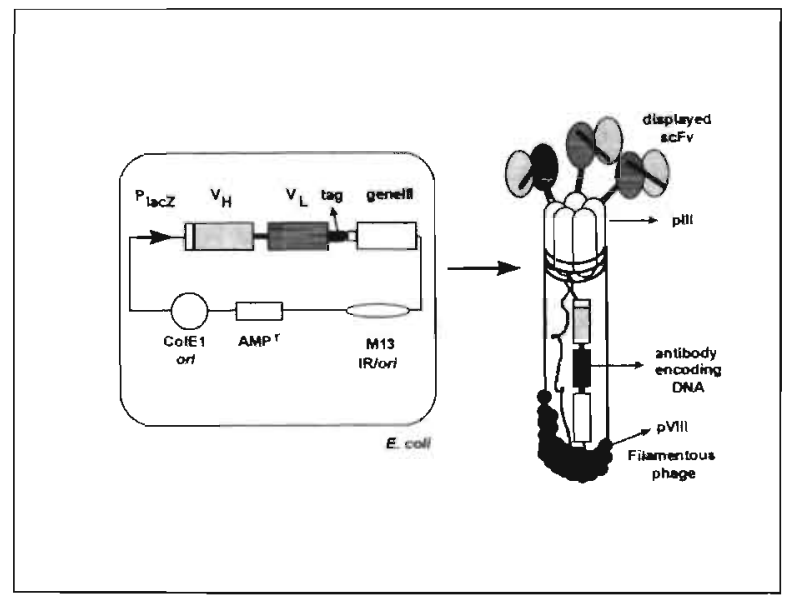

Fig. 1. Display of scFy antibodies on filamentous phage. Bacteria harbouring a phagemid vector, encoding the $\mathrm{V}_{\mathrm{H}}$ and $\mathrm{V}_{\mathrm{L}}$ immunoglobulin genes fused to gene III of filamentous phage $\mathrm{fd}$, are superinfecied with helper phage to drive production of phage particles carrying the $s c F v$, as a fusion product with the phage coat protein pIII. on the surface, and its encoding DNA inside.

DNA encoding the antibody-pIII fusion will be preferentially packaged into phage particles using a helper phage, such as M13KO7 or VCS-M13, which supplies all structural proteins. This includes also wild type gene III, which will compete with the phagemid-encoded gene III fusion for incorporation in the phage particles. In phagemid systems, a promoter is used (for example, derived from lacZ), which may be suppressed (with glucose) to decrease antibody-pIII expression during library amplification. This prevents some of the bias in the population caused by differential toxicity of antibody-pIII fusions. However, the introduction of an additional 
transcription terminator abolishes toxicity altogether [51]. For display of the antibody-pIII product, the repressor (glucose) is removed, leading to sufficient fusion product to derive 'monovalent' phage particles [48,50,52]. For display of Fab fragments [48.50,52] the heavy chain fraginent (Fd) or light chain $\left(\mathrm{V}_{\mathrm{L}} \mathrm{C}_{\mathrm{L}}\right)$ was fused to gene III and the partner chain was co-expressed separately in the periplasm.

Besides gene III, of which there are approximately five copies on each phage particle, antibodies may also be fused to the small major coat protein derived from gene VIII, present at approx. 2,700 copies per wild type phage particle (for review [53]). Display on one of the other minor coat proteins, pVI, as carboxy-terminal fusion, has been reported for cDNA libraries, but not for antibodies. Although gene III protein is involved in infection, the infectivity of antibodypIII-containing phage particles often appears not to be dramatically affected by the fusion. However, it has been reported that part of the antibody-pIII fusion is proteolytically cleaved at the fusion junction, providing a source of full-length, thus wild-type, pl[I [43,54]. Furthermore, besides fusion to full length pIII, fusions to sites downstream of gene ПII have been reported $[24,55,56]$.

The key feature of the display of ligands on the phage surface is the linkage of phenotype (surface displayed ligand) and ligand genotype, encoded within the phage genome [57]. This allows the enrichment of antigen-specific phage antibodies, using immobilized or labelled antigen. Phage that display a relevant antibody will be retained on a surface coated with antigen, while non-adherent phage will be washed away. Bound phage can be recovered from the surface, reinfected into bacteria and re-grown for further enrichment and, eventually, for binding analysis. This antigen-driven enrichment procedure may be used to isolate even the rarest phage antibody. In this respect, an important feature of the filamentous phage is its resistance to the severe elution conditions that are used for dissociation of ligands: the phage remains infective when treated temporarily with acid, alkali or with denaturing agents such as urea (for review see [57]).

Alternative display methods have been described, but none have matured as much as the filamentous phage system. These include display on other phage hosts ( $E$. coli bacteriophage lambda [58,59,60,61], or T4-phage [62]), on prokaryotic hosts or their extensions (flagelli, $E$. coli itself, LamB and OmpA etc.; for review, see [63]), on eukaryotic hosts or their viruses (yeast [64]), including retroviruses [65,66]. None of these systems have been used as extensively as filamentous phage and, to date, neither the construction of large antibody libraries nor the selection of high affinity antibodies has been reported.

\subsection{Library technology}

The success of antibody phage display hinges on the combination of the display and enrichment method, with the creation of large combinatorial repertoires of phage antibodies, which will be discussed first. 
Naturally occurring V-genes may be isolated from B-lymphocytes by application of PCR [37,38,39]. cDNA synthesized from total or poly-A RNA extracted from B-lymphocytes can be used as template for PCR; alternatively the rearranged chromosomal DNA is taken (reviewed in [57]). As a source for genetic material, peripheral blood lymphocytes can be used; or the spleen, lymph nodes, tonsils and bone marrow, with the choice depending on the type of library that will be made.

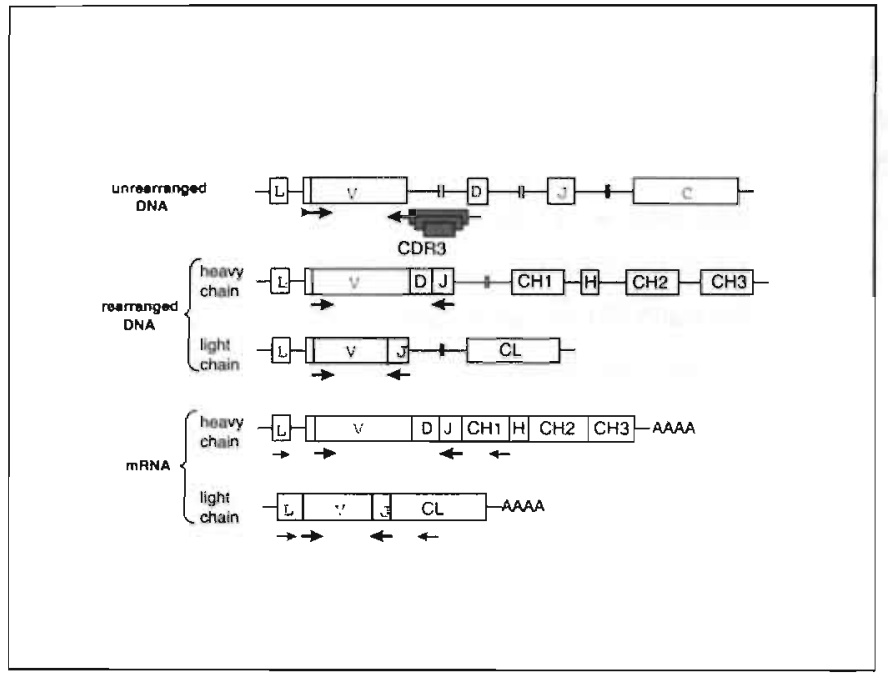

Fig. 2. Location of primers in antibody genes. Combinations of forward and backward primers that map in the signal, framework or constant regions may be used to amplify antibody $V$-genes from (un)rearranged DNA or mRNA.

Several sets of "universal" PCR primers, suitable for the amplification of large arrays of V. genes, have been described for repertoire cloning. As is shown in Fig. 2, antibody encoding DNA fragments can be amplified with different combinations of oligonucleotides, based on either the signal sequence, the $5^{\prime}$ and $3^{\prime}$ end of the variable genes, or on the constant region. The 5 ' and $3^{\prime}$ ends of the $\mathrm{V}$-genes are sufficiently conserved to allow the design of primers for the amplification of mouse variable regions [19,39], or human light and heavy chains [36]. Extensive sets of both family-specific and degenerate primers have now been described, for the construction of human [20,36,67], murine (also usable for rat) $[19,24,39,68,69]$, rabbit [70], or chicken $[71,72] \mathrm{V}$-gene repertoires.

For cloning as scFv- or Fv-fragments, the easiest route involves amplification of the $\mathrm{V}$ genes with oligonucleotides based on the FR1- and FR4-region, and cloning of these fragments into an appropriate display vector. The primers can be designed to contain restriction sites introduced within the antibody $\mathrm{V}$-sequence [40], or may be appended with cloning sites outside the V-region [19]. The choice depends very much on the cloning vector and strategy used. Cloning may be carried out in a stepwise, sequential fashion or may involve a 'splice overlap extension' ( $\mathrm{SOE}$ [73]) reaction to sow together $\mathrm{V}_{\mathrm{H}}$ and $\mathrm{V}_{\mathrm{L}}$ genes in scFv or Fab format before 
one-step cloning (reviewed in [57]). For the construction of Fab-repertoires, the introduction of isotypes of the $\mathrm{C}_{\mathrm{H}} \mathrm{l}$-gene, which are present in the mRNA, should be avoided, to prevent bias in the repertoire due to expression differences [74], and to simplify the inclusion of the disulphide bond between the heavy and light chain. Provision of this intermolecular disulphide bond is not strictly necessary; the pairing of heavy and light chain is sufficiently stable without this covalent bond. Indeed, Fab repertoires have been made by supplying the same $C_{H} l$ domain (often the human gamma- $1 \mathrm{C}_{\mathrm{H}} 1$ gene) in the display vector $[48,75,76]$. Fab repertoires are then constructed by cloning in the $\mathrm{V}_{\mathrm{H}}$ gene pool, and the complete light chain (lambda and kappa). again either in a stepwise fashion, or after SOE. An isotype-specific $\mathrm{V}$-gene may be amplified by using appropriately designed isotype-specific oligonucleotides during first strand cDNA synthesis or during V-gene amplification [20].

For cloning, originally, restriction enzymes were chosen that were frequently present in naturally rearranged $\mathrm{V}$-genes. such as PstI in the FRI of $\mathrm{V}_{\mathrm{H}}$, and BstEII in the FR4 of $\mathrm{V}_{\mathrm{H}}$, the advantage being that, at these positions, the naturally occurring residues were maintained in FR 1 and FR4 [39]. In due course, improved designs have appeared which involve the use of restriction enzymes that more rarely cut within antibody genes, on the basis of recognition of 8 bases (SfiI, NotI, AscI etc.) [24,77], or on the basis of a study of the frequency of restriction sites in a large database of $\mathrm{V}$-genes [78]. The use of SOE reduces the number of cloning steps, but is a technically demanding procedure, that, if not carried out carefully, may lead to loss of diversity due to excessive use of PCR.

Fig. 3 illustrates the construction of a library. B-lymphocytes. expressing the antibodies, serve as a source of V-genes. After RNA- or DNA-isolation, the V-regions are amplified and randomly assembled to create scFv cassettes. These are supplied by restriction sites at the $5^{\prime}$ and $3^{\prime}$ ends, and cloned into a phage(mid) vector for expression of fusions of scFv phage coat protein. This procedure will yield random combinatorial libraries (the original pairing of $V_{H}$ and $\mathrm{V}_{\mathrm{L}}$ will be lost).

\subsection{Selection strategies}

Phage antibody libraries are enriched for antigen-binding clones by subjecting the phage to repetitive rounds of selection, which includes binding. washing and elution steps, re-infection into bacteria and growth/rescue to re-express the antibody on the phage surface. This selection cycle is illustrated in Fig. 4A. (for a review, see [79]). Ideally, only one selection round should be required, but the aspecific binding of phage limits the enrichment that can be achieved per round and in practice two - four rounds are necessary.

In Fig. 4B, the most frequently used in vitro selection methods are described. The first selections of phage antibodies were achieved with affinity columns [19,43] (Fig. 4B, panel a) and later with antigen adsorbed onto plastic surfaces (Fig. 4B, panel b) such as en $z y m e-l i n k e d$ immunosorbent assay (ELISA) plates or "Immunotubes" [20.52], or antigen immobilised on 
BIAcore sensorchips [80]. In all cases, care must be taken to retain the conformational integrity of the antigen, e.g., sometimes antibodies selected against a protein adsorbed onto plastic may not recognise its native form. An indirect coating may be applied, using an antigen-specific

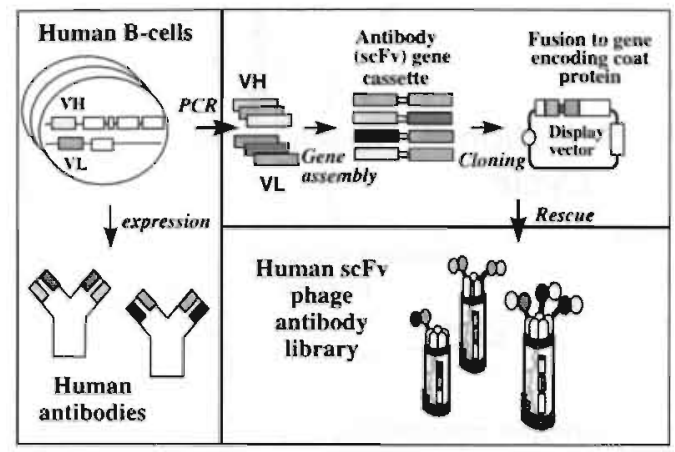

Fig. 3. Construction of a human antibody libiary displayed on phage. cDNA fragnuents encoding for the heavy and the light chain variable regions of the antibodies (VH, VL) are amplified from human B-cells by PCR and assembled. The assembled genes are inserted in a phagemid vector in frame with the gene encoding the coat protein plll. The vector is introduced into $E$. coli. After rescue with helper phage, the random combinatorial library of antibodies is displayed on phage.

antibody for capture [81]; non-specific Ig of the same species and isotype may be added during the selection procedure to avoid isolation of antibodies to this primary reagent. Adaptation of the panning method permits the selection of antibodies with a desired specificity, as with the epitope masking technique $[82,83]$, in which an immuno-dominant epitope was blocked by a mAB. To prevent the selection of cross-reactive antibody fragments, a competitor antigen that is highly homologous to the protein of interest may be added, to provide a competitive deselection [84]. Furthermore, the selection on antigen. which was displayed on the surface of bacteria has been described, which appeared, surprisingly, more efficient than when selecting on purified antigen [85].

These panning or column methods do not casily allow a quantification of the amount of antigen used and, thus, the concentration used during selection. This may be achieved by applying a powerful and, probably, now, the most frequently applied method, based on using labeled soluble antigen such as bintinylated antigen [86]. After incubation of phage mixes with the biotinylated antigen, phage bound to the antigen-biotin complex are retrieved with streptavidin-coated paramagnetic beads and a magnet (Fig. 4B, panel d). One of the advantages of this selection procedure is that the concentration used during the selection can be carefully chosen. During the selection of the phage library, the antigen concentration may be decreased to favour the enrichment of antibodies with a higher aflinity [87]. A minor disadvantage of this 
method is that antibodies to streptavidin will also be isolated, in particular, when non-immune libraries, are used; in cases where a high frequency of streptavidin binders are found, a depletion step with coated streptavidin should be performed.

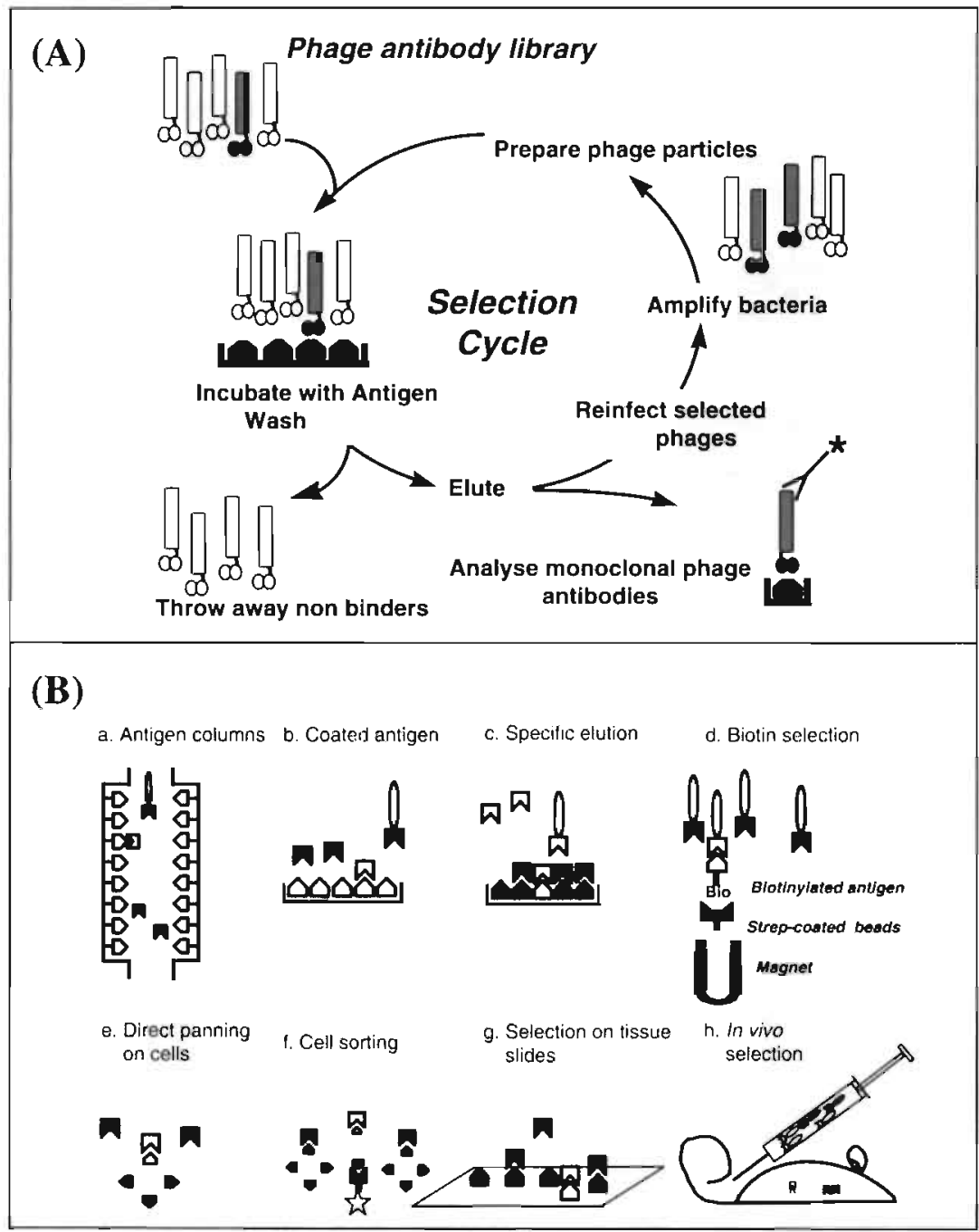

Fig. 4.(A) Procedures for selection with phage libraries. A phage antibody library is incubated with antigen and nonbound phage are washed away. Specific library phage is eluted and reinfected in $E$. coli for amplification and rescue. Phage particles from this amplified library are used to incubate with antigen in a second round of selection. After two to four rounds of selection, individual (monoclonal) antibodies are analysed.

(B) Selection strategies to obtain specific phage antibodies. Selection of phage libraries by flow through a column with immobilised antigen (a) or by panning on antigen adsorbed onte plastic (b). After washing. specific phage is eluted with acidic or basic solutions; alternatively, elution with an excess of the antigen is possible (c). To avoid conformational changes during coating, selection of specfic antibodies to biotinylated antigen in solution is more favoured. Bound and unbound phage antibodies are separated using streptavidin-coated magnetic beads (d). Selection on cells can be done directly by panning on cell monolayers or on cells in suspension; unbound phage are washed away by rinsing the flask or by centrifugation (e). Subtraction via FACS: the cells of interest are fluorescently labeled and separated from the others by cell sorting (f). Tissue- and organ-specific phage antibodies might be obtained by selections on tissue slides $(\mathrm{g})$ or by in vivo selection (h). 
Phage antibodies bound to antigen can be eluted in different ways, e.g., with (one step or gradients of) acidic solutions such as $\mathrm{HCl}$ or glycine buffers [52,88], with basic solutions like triethylamine [20], with dithiothreitol (DTT) when biotin was linked to antigen by a disulphide bridge [89], by enzymatic cleavage of a protease site engineered between the antibody and gene III [90], or by competition with excess antigen [19] (Fig. 4B, panel c) or antibodies to the antigen [91].

Selections on impure antigens are significantly more difficult, due to the problem of enriching phage antibodies specific for non-relevant antigens. Examples are antigens that cannot easily be purified from contaminants with similar properties, or cell surface receptors that can be functionally retained in lipid bilayers only. Direct panning on cell surfaces may be carried out on adherent cells grown in monolayers, or on intact cells in suspension (Fig. 4B. panel e). This may fortuitously select for antigen-specific phage antibodies, in particular, when using immune libraries in which the frequency of irrelevant phage antibodies will be lower [92,93]. When using panning with a large naïve repertoire [94] on a colorectal cancer cell line, we generated antibodies of all the expected types (Zijlema, Hoogenboom et al., unpublished): (1) antibodies recognising pan-cell markers, (2) antibodies binding to the cancer cell line only and (3) a series of antibodies binding to antigens related to the epithelial cell origin. For the latter antibodies, the exquisite epithelial cell origin of the antigen was confirmed by positive immunohistochemistry on colon tumor's derived from cancer patients. For most applications, depletion and/or subtraction methods have to be used to home in on antigen-specific phage antibodies, for example, cell sorting by flow cytumetry (Fig. 4B, panel f) $[25,95]$ or magnetic activated cell separation. In this case, the (excess of) non-selected cells serve to absorb irrelevant phage antibodies. A powerful selection was achieved by alternating between different sources of antigen [96], to home in on antigen-specific phage antibodies. To fully understand and appropriately design selection of phage antibodies on cell surfaces, systematic studies to compare the various selection methods [97.98] are required. This will be aided by establishing mathematical models for bio-panning, as was elegantly done for phage displaying peptides [99].

Further selections may be carried out on even more complex mixtures of antigens, for example, on tissue sections (Fig. 4B, panel g; Roovers, Hoogenboom et al., unpublished), or whole tissues [100] which will yield, depending on the type of library used, antibodies to cell surface. intracellular and stromal compartments. This may be of particular interest when targeting rare antigens expressed in vivo in certain pathological situations, and accessible on tissue sections only. In vivo selections, in which the phage repertoires are injected directly into the blood of animals and certain target organs or tissues are collected (Fig. 4B, panel h), may reveal phage antibodies specific for organ/tissue-specific endothelial cell antigens, as has been demonstrated for peptide phages [101].

Selection from phage antibody libraries provides a new tool for the isolation of novel self antigens. such as tumor- or disease-associated antigens. Both the de novo combined $\mathrm{V}$ domain 
pairs in naïve and synthetic antibody libraries (but particularly the latter) are not shaped by the constraints of the immune system, and have not been skewed against anti-self by in vivo tolerance mechanisms. Therefore, the chances to detect unique self antigens and epitopes are improved dramatically compared with the immunization-dependent hybridoma-based antigen screening. To date, this application has been used for probing lymphocyte- [95] and tumor cell surlaces [92], yielding a number of promising [102], but, as yet, uncloned cell-type-specific antigens. The characterization of the antibodies is hampered by the frequent lack of reactivity in inmunostaining [42], the antibody's low affinity [95], or the low abundance of the cell surface antigen.

In general, the obtained enrichment factors during panning vary between 20 and 1000 per round $[20,43]$, but with high affinity antibodies, they may reach $10^{5}$ per round (Roovers and Hoogenboom, unpublished). Depending on the display vector used, the percentage of recovery of a selected clone may vary from $0.01-0.1 \%$ to $30 \%$ per round. These factors are dependent on the affinity of the antibudy, the display efficiency (i.e.. the number of antibody copies per phage). the concentration of the antigen, and the physical method of selection (washing steps, elution method, etc.) $[54,79]$. In theory, the number of selection steps that can be performed is unlimited, thereby allowing successful selection even with minimal enrichment factors/recoveries. In practice, a minimal efficiency is required, in order not to loose phage antibodies, in particular, when selecting from large libraries (see also below).

The selection procedure will enrich binders from the phage library. To select those phage antibodies with the best affinity, decreasing amounts of antigen should be used, selections should be carried out in solution rather than by avidity-prone panning, and sensitive and accurate affinity screening methods, such as a BIAcore-based affinity screening, should be available.

The enrichment factor is influenced also by the background binding of non-specific phage particles, which necessitates reiteration of the selection procedure. This may be by-passed in some applications by repeating the selection of eluted phage immediately without reamplification [103]. Two alternative systems have been described, with the aim of reducing the selection step to just one round $[104,105]$. In both methods, the antibody library is made using non-infective phage particles, by deleting part of the gene III protein. Infection will be restored by binding a fusion protein consisting of the cognate antigen and the two first amino terminal domains of gene III, which are responsible for pilus binding [106]. The fusion product may be supplied in vitro; infection of the antibody-displaying phage will be mediated in a small concentration window [107]. In the in vivo SIP system (selective infection of phage, [107]), both antibody and antigen are encoded on the same phage genome. Applications of this promising system are hampered by the inefficiency of the restoration of infectivity; even when using an antibody-antigen pair of high affinity. infection using SIP remains at least 10.000 -fold less efficient than when using phage carrying the wild-type gene III product. It remains to be seen if these selection systems will be able to replace the more controlled in vitro selection methods. 


\subsection{Screening assays}

After the selection procedure, a fast and robust screening assay for binding of poly- or monoclonal phage antibodies is applied, ranging from a simple ELISA with coated antigen, bioassays for screening for direct neutralization upon binding [108], to (for cells) whole cell ELISAs (Lutgerink, Hoogenboom et al., unpublished). Fluorescence-Activated Cell Sorting (FACS) [100] or immunocyto- or histochemistry [109,110,111,112]. For speeding up screening procedures, phagemid vectors that incorporate a dual purpose, obtaining monovalent display of scFv or Fab fragments, and producing soluble antibody fragments for screening [48,56], have been developed. In such systems, an amber codon was positioned between the antibody and pIII gene; in suppressor $E$. coli strains, this allows expression of the fusion product, for incorporation in the phage coat; in non-suppressor strains, this amber serves as a stop codon, yielding soluble antibody fragments. A variety of tags has been described that are appended to the antibody fragment for detection, including the myc-derived tag recognised by antibody $9 \mathrm{E} 10$ [20], and the Flag sequence [113]. This set-up will allow the use of unpurified phage antibodies or antibody fragments, present in crude supernatant or periplasmic extracts, for screening assays.

Ideally, the screening assay is linked as closely as possible to the final read-out test for the antibody. Frequently for this, the antibody fragments will need to be purified; fortunately histidine-tags for the popular immobilised metal affinity chromatography (IMAC) [114] are compatible with phage display [115]. For reformatting selected antibodies in alternative formats, fast recloning methods are needed for screening large numbers of clones. An eukaryotic expression vector recently described may be used for one-step recloning of V-genes derived from any phage repertoire, and cloned for expression as Fab or whole antibody, or for targeting to different intracellular compartments $[78,116]$. Fast read-out systems as well as binding tests are required, in particular for screening methods where the influence of affinity or kinetic behaviour is unclear or less important than the precise epitope that is recognised, e.g. virus or cytokine neutralization assays. For example, methods to quickly test the neutralization of growth factor receptors by Fab cross-linking have been reported [108].

We have recently obtained evidence that ligand-displaying phage themselves may act as receptor agonists (Rousch, Hoogenboom et al., submitted), a feature which will simplify screening for phage-based ligands in search of a(nta)gonistic lead compounds dramatically. But with large libraries at hand. we may go beyond the in vitro binding interaction itself. and select for a particular function. Examples of previously demonstrated functional selection methods are found in retrieving catălytic antibodies [117,118]: as yet unexplored routes are selections for cell internalization [119]. cell survival or killing (induction of apoptosis) upon ligand binding, viral entry, receptor cross-linking and receptor triggering (or its blocking). 


\section{Antibody repertoire cloning}

The choici on which type of phage antibody repertoire to use depends very much on the application and the required aftinity of the antibody. Ideally, we would have available universal, antigen-unbiased libraries, from which ultra-high affinity antibodies may be selected directly. Right now, two types of such 'single-pot' libraries have been described, the naive and synthetic antibody libraries (reviewed in [57].) From both types of libraries, antibodies against any chosen antigen may be selected, but the affinities are not always sufficient for the application. The alternative is to start with antigen-biased phage antibody repertoires, using $V$-genes from immunised B-cells. or to affinity mature the antibodies. We will discuss the differences between these repertoires, summarised in Fig. 5 (see also [79]), starting with the immune repertoire.

\begin{tabular}{|c|c|c|c|c|}
\hline $\begin{array}{l}\text { Equivalent cell } \\
\text { type in the in } \\
\text { vitro Immune } \\
\text { system }\end{array}$ & $\begin{array}{l}\text { Antibody } \\
\text { repertoire }\end{array}$ & Advantages & Disadvantages & Appllcation \\
\hline $\begin{array}{l}\text { Plasma/ } \\
\text { memory cells }\end{array}$ & $\begin{array}{l}\text { - Immune using } \\
\text { rearranged V- } \\
\text { genes from IgG } \\
\text { pool } \\
\\
\text { - Naive using } \\
\text { rearranged } \\
\text { V-genes from } \\
\text { IgM pool }\end{array}$ & $\begin{array}{l}\text { - Ag dependent } \\
\text { repertoires } \\
\text { - Antibodies biased for } \\
\text { high affinity } \\
\text { - immunodominant } \\
\text { epitopes } \\
\text { - Originally controlled for } \\
\text { unwanted self } \\
- \text { One repertoire } \\
\text { construction for } \\
\text { different antigens }\end{array}$ & $\begin{array}{l}\text { - Uncontrolled } \\
\text { contents } \\
\text { - High aftinity } \\
\text { dependent on } \\
\text { library size: } \\
\text { - Specificity originally } \\
\text { biased against } \\
\text { self }\end{array}$ & $\begin{array}{l}\text { - Analysis of naturai } \\
\text { immune response } \\
\text { - Source of V-genes for } \\
\text { chain shuftling } \\
\text { - In vivo depleticin of } \\
\text { unwanted specificities } \\
\text { - Any specificity with } \\
\text { the same libirary }\end{array}$ \\
\hline $\begin{array}{l}\text { Pre B cell } \\
\text { PI }\end{array}$ & $\begin{array}{l}\text { Synthetic, using } \\
\text { clonecl. } \\
\text { unrearranged } V \text {. } \\
\text { gene segments }\end{array}$ & $\begin{array}{l}\text { Controlled } \\
\text { contents } \\
\text { One repertoire } \\
\text { construction for } \\
\text { different antigens }\end{array}$ & $\begin{array}{l}\text { Affinity dependent } \\
\text { on library size }\end{array}$ & $\begin{array}{l}\text { - Any specificity with } \\
\text { the same library } \\
\text { - Systematic affinity } \\
\text { maturation methods }\end{array}$ \\
\hline
\end{tabular}

Fig. 5. Comparison between different antibody repertoires (see text for details).

\subsection{Immune repertoires}

For immune libraries, V-genes are derived from the IgG mRNA of B-cells of an immune source, e.g. immunized animals or, in some instances, human immune B-cells. An immune phage antibody library repertoire will be enriched in antigen-specific antibodies, some of which will also have been affinity matured by the immune system $[19,120]$.

This method gives access to more and sometimes better antibodies than when working with hybridomas. For example, from an immune murine phage antibody library, Chester et al. [121] identified an anti-Carcinoembryonic antigen (CEA) antibody with an affinity that was 
substantially higher than ever obtained with the hybridoma technology. There are plenty of other examples in the literature of other murine immune phage antibody libraties $[96,122,123]$. Immune libraries were used to retrieve antibodies to 'difficult' antigens, including native T-cell Receptor (TCR)-V $\alpha$ [124] and specific major histocompatibility complex (MHC)/peptide complexes [96]. Recently, even the derivation of repertoires from rabbit [70] and chicken [7]] were reported, both describing the isolation of high affinity antibody fragments.

Disadvantages of this strategy, however, include the time required to immunize animals, the unpredictability of the immune response to the antigen of interest, the lack of immune response to some antigens (self antigens or toxic molecules), and the restriction in generating human antibodies. A major drawback of this method is that, for every new antigen, a new phage antibody library has to be created, which adds one - three months to the procedure. Therefore, when large naive or synthetic antibody libraries are available, the use of immune libraries should be avoided [79].

In the longer term, there may be a few occasions where this route will still be useful, i.e. for the analysis of the humoral immune response in man. Naturally occurring high-affinity human antibodies, for example, those present after viral infection or in patients with auto immune diseases or cancer, may be cloned by phage display, with caution, to aid in identifying the antigen or epitope(s) involved in the immune response. The scrambling of $V_{H}$ and $V_{L}$ genes in the process of the combinatorial library construction makes it difficult to discriminate 'original' $\mathrm{V}_{\mathrm{HI}}-\mathrm{V}_{\mathrm{L}}$ combinations from de novo formed pairs. The likelihood that original pairs may be retrieved is very low (theoretically, the library size would be at least $10^{12}$ to retrieve original $\mathrm{V}_{\mathrm{H}} / \mathrm{V}_{\mathrm{L}}$ pairs from an immune repertoire of $10^{6}$ lymphocyles), although it appears that the heavy chain often dominates the specificity [125]. If it is necessary to maintain the original pair, "in-cell PCR', in which $V_{H}$ and $V_{L}$ genes are assembled within the cell [126] before cloning, may be used, or the B-cells may be enriched on antigen $[127,128]$ and/or cloned by limiting dilution, before amplification of the $\mathrm{V}$-genes.

Phage display has been used extensively to study the characteristics of self-antibodies in auto immune diseases (for recent examples, see $[125,129]$ and references therein). A well studied example of humoral responses in viral diseases involves the immune libraries prepared from the bone marrow of human immunodeficiency virus type 1 (HIV-1) seropositive donors. This library appeared to form a rich source of potent neutralizing antibodies to HIV-1, but also to respiratory syncytial virus (RSV) and herpes simplex viruses HSV-1 and HSV-2 (for review, see [130]). While most high affinity Fabs were selected against the CD4-binding site of the HIV-1 envelope protein gp120, other antibodies directed to different epitopes were jsolated using the epitope masking technique or by using peptides [83]. In this thesis, we will also describe the application of immune repertoires, of murine and human origin, for the selection of HIV-I specific antibody fragments. 
In a second application of immune libraries, the immune response itself is used to remove irrelevant antibodies, thus biasing the $\mathrm{V}$-genes before cloning against certain antigens. Animals can be made tolerant to certain irrelevant antigens, after which the relevant antigen as a mixture with irrelevant antigens is used for the immunization. This combination of depletion and enrichment. in vivo (tolerization and immunization) and in vitro (depletion of phage antibodies before selection on antigen), is extremely powerful, as exemplified by the isolation of human anti-tumor cell antibodies from phage repertoires of cancer patients immunized with autologous tumor cells [92], or from their tumor-draining lymph nodes [131].

\subsection{Naive repertoires}

The primary immune response involves a large array of IgM antibodies that recognize a variety of antigens. The murine naive repertoire has been estimated to contain $<5 \times 10^{8}$ different B-lymphocytes, while the human repertoire may be a hundred to a thousand limes bigger [57]. This array of antibodies may be cloned as a 'naive' repertoire of rearranged genes, by harvesting the V-genes from the IgM mRNA of B-cells of unimmunized human donors [20], isolated from peripheral blood lymphocytes (PBLs), bone marrow, spleen cells, or from animal sources [132]. The first of such 'single-pot' repertoires [20] was made from the PBLs of two healthy human volunteers and contained $3 \times 10^{7}$ clones. From this pool, antibodies to over 25 different antigens were isolated [20.89,93]. The antigens were either "foreign", such as turkey egg white lysozyme, bovine serum albumin and the hapten 2-phenyloxazol-5-one (phOx) [20], or "self", such as thyroglobulin, Carcinoembryonic antigen, mucin, CD4 and tumor necrosis factor $\alpha$ [89]. When probed with entire red blood cells, scFvs directed against antigens to blood group $\mathrm{B}, \mathrm{D}$ and $\mathrm{E}$ [93] were isolated. The average affinity of these antibodies was similar to the affinity of antibodies seen in the naive primary immune response. This appears to be sufficient to allow reactivity in Western blot, ELISA, FACS analysis, etc. [109]. Recently, much larger scFv repertoires have been made by 'brute force' cloning [94] (using $100 \mu \mathrm{g}$ of vector ligated to $10 \mu \mathrm{g}$ of insert transformed into bacteria by several hundred electroporations) yielding $1.4 \times 10^{10}$ independent antibody clones. As source of lymphocytes PBLs, tonsil B-cells and bone martow B-cells were taken from 43 non-immunized individuals. From this library, antibodies with affinities typical for a secondary immune response were isolated (average $K_{a}$ around $10^{8} \mathrm{M}^{-1}$ ), against all of the antigens tested, with two antibodies, against haptens diethylenetriaminepentaacetic acid (DPTA) and fluorescein, in the subnanomolar range [94]. For a direct comparison with the smaller sized library from Marks et al. [20], the increase in library size by a factor of 200 led to an apparent 100-fold decrease in off-rates for anti-phOx antibodies. Even larger sized naive Fab repertoires may be made by using the in vivo recombination system based on Cre/loxP recombination system ([133] see below).

By brute force cloning. we generated a huge Fab displayed, human repertoire with $4.1 \times 10^{10}$ clones, which might be used for the generation of high affinity antibodies for diagnostic 
applications. The construction and selection with a number of antigens will be described in this thesis (Chapter 7).

Thus, neither immunization nor affinity maturation are needed for the generation of high affinity (human) antibodies. Major advantages of using very large naïve single-pot repertoires therefore are as follows: (1) one library can be used for all antigens, (2) human antibodies can be isolated, (3) antibodies to self, non-immunogenic or toxic substances can be generated, (4) antibody generation takes two - four rounds of selection (approximately two weeks), and (5) when very large repertoires are available, high-affinity antibodies are isolated directly.

The disadvantages are (1) the lower affinity of the antibodies when smaller-sized repertoires are used, (2) the time and effort it takes to construct these repertoires, in particular, when using the Cre/LoxP recombination system (see section 1.3.4) and (3) the largely unknown and uncontrollable contents of these naive libraries. In particular, the positive and negative selection by the immune system, the potentially limited diversity present in the IgM repertoire, its possible bias due to unequal expression of the different $\mathrm{V}$-gene families and the unknown immune history of the B-cell donor. influence the contents and quality of the library. Therefore, an alternative method, based on the controlled addition of defined and chosen V-gene building blocks to antibody repertoires, was developed, i.e., the synthetic antibody library.

\subsection{Synthetic repertoires}

An antibody's specificity resides in the six complementarity determining regions (CDRs) that shape the antigen combining site. The two variable domains that compose this site are generated by the immune system by recombination of genetic elements, creating an immense variety of antibodies. There are three sets of genetic elements $\left(\mathrm{V}_{\mathrm{H}^{-}}, \mathrm{D}\right.$ - and $\mathrm{J}_{\mathrm{H}^{-}}$-regions) for the heavy chain and two sets for the light chain (no D-segment). The V-segment encodes the first two CDRs. the third one is the product of the junction of $V / D / J$ for the heavy chain or $V / J$ for the light chain (for review, see [134]). This knowledge has paved the way for the creation of antibodies entirely outside their natural host. To construct a synthetic antibody library, V-genes are assembled by introducing a predetermined level of randomization of CDRs (or possibly also bordering FR-regions) into, ideally, germline V-gene segments [21]. One of the main advantages over naive repertoires is that, in such synthetic repertoires, the contents, local variability and overall diversity of the library will be controlled and defined.

The areas of synthetic diversity ought to be chosen to correspond to areas of most natural sequence diversity of the primary antibody repertoire. From structural studies, it has become apparent that five of the six CDR regions (all but the CDR3 of the heavy chain, the most central to the antigen combining site of all CDRs) have limited structural variation, and frequently follow a certain canonical fold [135]. There is no apparent consensus in the heavy chain CDR3 (except for the J-segment-encoded residues at the carboxy-terminus), with an estimated potential diversity of $10^{23}$ different sequences for this loop only [136]. Thus, the CDR3 of the heavy chain 
is the most diverse loop, in composition, length (ranging from 2 to 25 residues) and structure, and this is the CDR region that should be partially or completely randomized using oligonucleotide-directed mutagenesis or PCR-based methods, for constructing synthetic repertoires.

Several synthetic libraries have been designed with the heavy chain CDR3 sequence encoded by oligonucleotide primers encoding a stretch of randomised amino acid residues. In the first synthetic antibody library constructed in Cambridge, UK, a human scFv repertoire was made containing $2 \times 10^{7}$ members, in which 49 cloned germline $V_{H^{-}}$-genes were provided with a "random" $\mathrm{V}_{\mathrm{H}}-\mathrm{CDR} 3$ loop with five or eight residues, combined with a fixed light chain [21]. From this repertoire, many antibodies to haptens, and one against a protein antigen, were isolated, delivering the first proof of concept for the "synthetic antibody". Subsequently, the diversity in the CDR3-regions was expanded to cover lengths ranging from four to twelve residues, to match the natural length diversity of this loop better [109], and eventually 47 light chain segments with partially randomized CDR3 were added [76]. In the final repertoire, nearly 100 cloned, human V-gene segments were used, covering over 95 ic of the segments used in the natural human repertoire and, therefore, resembling the human primary antibody library in the diversity of all but the heavy chain CDR3 [71]. The repertoire, with $6.5 \times 10^{10}$ members was made by in vivo recombination (see below), and contained hapten- or protein-specific Fabs with nanomolar affinities, typical for antibodies from a secondary immune response.

Several other synthetic or semi-synthetic repertoires have been made, varying from the most simple approach being to use one rearranged V-gene pair [137], using a defined set of V-gene segments (derived from the first synthetic repertoire [21]) extended with six light chain segments [138], to using an undefined mix of human germline V-gene segments, with randomised CDR3s [139], to diversifying all three CDR loops in one V-gene segment $[140,141]$. In the first approach, only the full CDR3 region (with sixteen amino acids) of the heavy chain was randomised. limiting the diversity of the repertoire in all other loops and creating a library resembling a constrained peptide library positioned on an antibody scaffold [137]. In the last example of the series, it may be expected that randomising the CDR loops other than the CDR3 of the heavy chain, without attempts to retain the residues involved in the canonical fold of these CDRs, may lead to a substantial fraction of the phage antibodies being misfolded, if expressed at all. Although some antigen-specific antibodies can still be selected from such libraries [140], they may run the risk of being much more immunogenic than antibodies with more naturally appearing $\mathrm{CDR}$ regions.

Clearly, many different synthetic library concepts will work to create antibodies of intermediate affinity. However, due to limitations in transformation efficiency, the total theoretically designed diversity normally exceeds practically accessible diversity. For example, when using mutagenic oligonucleotides to randomize six codons (with at each triplet, the first two base positions $\mathrm{A}, \mathrm{G}, \mathrm{C}$ or $\mathrm{T}$, and the last $\mathrm{G}$ or $\mathrm{C}$ ), one billion combinations arise. It is 
therefore better to limit the diversity to one or two CDR areas in the primary repertoire, ideally the heavy and light chain $C D R 3 s$, since these are naturally the most diverse sequence. After selection, the other CDR loops may be targeted with diversity, while conserving within these $\mathrm{CDR}$ regions the canonical positions governing their main-chain conformation. The next section will deal with how larger repertoires may be made, such that a larger number of antibody variants $\left(10^{12}\right)$ may be screened for activity.

\subsection{Making large repertoires}

Typical library sizes made by direct cloning of antibody genes as PCR products are around $10^{6}-10^{8}$. For these libraries, the total number of different V-genes outnumbers the number of independent colonies that is obtained by direct transformation of $E$. coli. It can be argued on theoretical grounds that, the larger the immune antibody repertoire is, the higher the chance will be to retrieve an antibody with a certain threshold affinity lor a given epitope [142], and the higher the chance of finding antibodies with higher affinity. Indeed, from naive or synthetic repertoires with much larger total library size $\left(10^{9}-10^{10}\right.$ clones), antibodies with affinities typical of the secondary immune response were directly isolated [76.94]. This section reviews how to create large size repertoires.

Besides 'brute force' cloning (see section 1.3.2), repertoires may be made larger than average by cloning the antibody genes as purified restriction fragments (Hoogenboom, unpublished). This procedure involves the separate cloning of $V_{H}$ and $V_{L}$ gene pools that had undergone PCR. followed by a second cloning step in which one of the repertoires is cloned as a digested restriction fragment into the phagemid containing the other repertoire (altematively SOE may be applied. provided that very long (>100 bases) overhangs are provided to allow efficient cutting of the assembled V-genes). Due to the increase in efficiency of ligation with well cut DNA fragments, this method has generated libraries with $10^{9}-10^{11}$ members (de Haard and Hoogenboom, unpublished).

The ceiling of the size of phage displayed repertoires can be more dramatically extended by applying a combination of the dual antibody cloning strategy [48] and a smart in vivo recombination system [133]. This involves the separate cloning of heavy and light chain repertoires: the light chain repertoire is cloned into a phage vector carrying two short recognition sites (loxP) for Cre recombinase, one of which is in frame with gene III, while the heavy chain Fd repertoire is cloned into a plasmid bordered by the same two loxP sites [133]. By using a wild type and a mutant loxP site that cannot recombine, the heavy chain will not be recombined out, but may be targeted to recombine with the identical sites based on the phage genome. After infection of bacteria carrying these plasmids with the light chain-containing phage, the bacteria are superinfected with bacteriophage $\mathrm{Pl}$, to produce Cre recombinase. This enzyme mediates recombination between the identical loxP sites and, thus, the transfer of the heavy chain to the phage genome. The progeny is a repertoire of Fabs on phage, the heavy chain of which is fused 
to pIII. The diversity is now limited only by the number of bacteria that can be infected, thus, $10^{12}$ in shaker flasks, but $10^{14}$ when using fermentors (providing that the combined potential initial heavy and light chain diversity is larger, i.e. $10^{6}$ for $V_{L}$ and $10^{9}$ for $V_{H}$ ). From a synthetic antibody library assembled from nearly $100 \mathrm{~V}$-gene segments, with randomized CDR3s, with over 60 billion clones, indeed antibodies with nanomolar affinities were isolated [76]. Increasing the library size thus results in the isolation of higher affinity antibody fragments, with affinities typical for in vivo matured antibndies, in spite of the absence of somatic mutations in the CDR 1 and $C D R 2$ regions of these antibodies.

Besides for antibody repertoires, this in vivo recombination strategy was successfully used for the assembly of a very large peptide repertoire ( $>10^{11}$ members) [143]. Two separately cloned 10-mer peptide repertoires were combined, in a similar way to the heavy and light chain repertoires, via in vivo recombination, onto one genome. In this instance, a trick had to be found to create one linear peptide library comprising combinatorial variants of the two starting libraries. For this, the loxP site was introduced into a ribosomal self-splicing intron. from Tetrahymena thermophila, which, after recombination, remained the only sequence separating the two 10-mer libraries. In vivo splicing at the RNA level then removed most of this intron, leaving only five 'residual' amino acids, separating the two randomised regions [143]. Such clever systems may also be used for creating large scFv (or, with appropriate design, other protcin) repertoires, in which the intron is placed in between $V_{H}$ and $V_{L}$.

The larger the library is, the higher the chance will be of retrieving higher affinity phage antibodies from the library, in theory [142]. However, there are physical limitations on the enrichment that may be achicved in one round of selection, which limits the size of accessible genetic diversity. This is illustrated by an example. After selecting on antigen with $10^{13}$ infectious phage particles from a library with over $10^{9}$ different antibody variants, typically approx. $10^{6}$ phage clones are recovered. This indicates that an enrichment of 1000 -fold (or recovery of $0.1 \%$ ) is required to select the one rare antigen-specific antibody in this population. If the enrichment factor is lower (which will be the case for many of the antibodics), there is a substantial chance that the clone is lost. Thus, with enrichment factors never reported to be higher than $10^{5}$ per round. and with $10^{6}$ clones eluted in the selection, the total genetic diversity accessed is at most $10^{11}$ clones. If selection conditions are so stringent that very few phage particles are recovered in the first round (typically for, for example, cell sorting via FACS), chances are that different subsets of antibodies will be selected every time this first round is repeated. It. therefore, appears that accessing even larger libraries will not generate even better antibodies. It becomes instead more crucial to optimise the quality of the displayed antibodies (with regard to display levels), and the selection procedures itself.

The construction of even larger libraries may be possible, one day, using a system that bypasses in vivo amplification and phage-background. This is based on the use of in vitro polysomes, in which mRNAs are transcribed in vitro from short DNA sequences and translated 
in cell free systems [144]. The polysome complex containing the encoding mRNA and are translated amino acid sequence are utilized for selection with a ligand. The mRNA from selected polysomes is converted into cDNA and used for the next transcription, translation and selection round. The fact that antibodies may also be in vitro translated to form functional molecules, reported in several cell-free translation systems $[145,146]$, is promising; however, it remains to be seen whether these in vitro methods can compete with the rather robust in vivo display technologies.

\section{Perspectives}

\subsection{Perspectives on selection technologies}

The use of $E$. coli as the expression host and filamentous phage as the display host have made antibody engineering fast and relatively simple. The use of very large naïve or synthetic libraries will yield antibodies with similar affinities to the ones generated with immune libraties, but much faster. For example, the affinities of the best antibodies directed to the hapten phOx, selected from an immune repertoire [19], were of the same range as those isolated from a very large synthetic repertoire [76]. Thus, the availability of pre-made large collections of nonimmune repertoires has superseded the use of immune repertoires [79]. It should be carefully considered if immunization and the creation of animal-derived antibody libraries is required, and also whether or not such experiments can be justified from an ethical point of view.

Overall, the impression now may be that slightly better antibodies are generated from naive [89] than from synthetic repertoires [76], however, the advantages in using synthetic antibody libraries [79] will eventually reduce primary naive libraries to useful but, in time, transient, sources of V-genes. A drawback of these methods is that the construction of libraries is technically difficult, but it is not unlikely that within a few years very large repertoires will be commercially available, like many other cDNA and genomic libraries, and peptide phage libraries. If the selection efficiency of phage libraries may be improved, for example, by using the infection-dependent methods such as SIP, multiple rounds of panning and replication may be avoided, and such process may even lend itself to being automated. Perhaps eventually the technology will lead to the development of a "panning-machine", with which every researcher will select a set of high affinity monoclonal antibodies against his or her favourite antigen or epitope, within the course of a few days.

The use of phage antibody libraries as a drug discovery tool has just begun. Interesting new methods for guided selection from crude mixtures, including "pathfinder" (Osbourn, Johnson et al., pers. comm.), will extend the scope of application of these libraries tremendously. Combining the design and generation of millions to billions of different ligands, with a functionbased selection procedure rather than selection for binding, will open even more challenging 
applications of this inspiring technology, and provide us with a new handle for drug discovery well into the next decade.

\subsection{Perspectives on antibody engineering}

By using phage display, we can generate completely human antibodies, and the provision of the V-genes facilitates further engineering of the antibody's characterislics. With this technology, antibody engineering for the first time may be used to design antibodies from scratch, with an option to choose their building blocks, affinity (up to the picomolar range). format (size and valency) and effector function (natural ( $\mathrm{IgG}$ ) or novel (enzymes etc.)). With these antibodies and derivatives, the role of affinity and dissociation rate, size and valency might determine which molecular format will be applied in a certain in vitro diagnostic. Tailor-made reagents, therefore, will be generated for in vitro but also for in vivo diagnosis and finally for therapy.

\section{Aim of the study}

For the development of in vitro diagnostics, monoclonal antibodies play an important role. Antibody repertoire technology seems to be an attractive alternative for hybridoma technology, since it allows the rapid isolation of monoclonal antibody fragments, and, as a consequence of the availability of the encoding antibody genes, the subsequent engineering of affinity, avidity and fusion to reporter enzymes. This thesis aims at the evaluation of repertoire technology for the generation of antibody fragments, useful as diagnostic reagents. The major targets in terms of the product range of the diagnostic company Organon Teknika were chosen, since it enables a direct comparison on epitope recognition and affinity with traditional monoclonal antibodies, that are used in products.

Antibody engineering was set up in at Organon Teknika after the first reports demonstrating the functional expression of antibody fragments in $E$. coli [7,8], but especially the publication of Ward and colleagues [40] in 1989, in which the idea of repertoire cloning was launched, gave the inspiration to start. As many others did in those days, a hybridoma derived monoclonal antibody (directed against the hormone human chorionic gonadotropin (hCG)) was used as a model. since combining only one heavy chain with one light chain variable region fragment would always: yield a functional scFv. Unfortunately it did not, and even increasing the number of monoclonal antibodies for the conversion in bacterially expressed frigments did not improve the outcome. The problems were caused by a mutation in framework 1 of a certain family of heavy chains, which was introduced by the oligonucleotide primers used for amplification of the V-regions. Chapter 1 summarises the identification of the mutation by application of engineering technology, and the analysis of the effect on folding of the antibody. Chapter 2 describes a primer induced mutation within FRI of a certain light chain family, which does not affect the 
overall folding found for the heavy chain mutation, but probably dispositions the CDR $I$ of $V_{L}$ and thereby affects antigen binding. For the quantification of the hCG-sc.Fv's, having the complete wild type sequence or with introduced mutations, we developed a method based on surface plasmon resonance, that is described separately in Chapter 3.

The solution for the non-functional, monoclonal antibody derived scFv's, was given in 1990 by the display of antibody fragments on filamentous phage, described by McCafferty and colleagues [43]. Using the hCG $\mathrm{mABs}$ as starting point small repertoires were constructed, with sets of primers for amplification of the V-regions, from which clones expressing functional scFv's were selected by biopanning on hCG, and from which the destructive mutations could be deduced. At that time, only two years after the introduction of immune repertoires in 1991 [19], it was just a small step to by-pass hybridoma technology and use repertoire cloning for the generation of monoclonal antibodies. In Chapter 4 anti-hCG antibodies, obtained by selection from a murine combinatorial library, were evaluated. The specificity as well as the affinity were studied in detail, and both characteristics were compared with those of monoclonal antibodies, which find their application in diagnostic assays. Both aspects are crucial for the development of a sensitive and rapid, hCG specific immuno-assay.

Repertoire technology was further applied in the context of HIV-1 detection. Chapter 5 describes the isolation and characterisation of anti-capsid protein (p24) scFv's from a library, made from an immunised mouse. Based on the article, published in 199] by Marks and colleagues [20], it was decided to construct a human immune repertoire using the lymphocytes from an HIV-infected individual, thereby superseding the immunisation of mice. The selection and characterisation of the obtained anti-envelope antibodies are discussed in Chapter 6 .

Finally, the application of naive repertoires, already described in 199] [20], but improved significantly in 1996 [94], eventually avoids immunisation and the repeated construction of immune repertoires. In 1997 a huge human naïve repertoire was generated using Fab as the. displayed fragment, and a large panel of antibodies was selected against the hapten 2phenyloxazol-5-one (phOx) and a number of protein antigens. Chapter 7 describes the construction and the successful selection of antibodies with unique specificities against the diagnostically relevant glycoprotein hormones hCG, hLH and hFSH from this repertoire. 


\section{References}

[1] Kohler, G. and Milstein, C. (1975) Continuous cultures of fused cells secreting antibody of predefined specificity. Nature 256, 495-497.

[2] Lilley, G. G., Dolezal, O., Hillyard, C. J., Bernard, C. and Hudson, P. (1994) Recombinant single-chain antibody peptide conjugates expressed in Escherichia coli for the rapid diagnosis of HIV. J. Immunol. Methods 171, 211-226.

[3] Coia, G.. Hudson, P. J. and Lilley, G. G. (1996) Construction of recombinant extended single-chain antibody peptide conjugates for use in the diagnosis of HIV-1 and HIV-2. $J$. Immunol. Methods 192, 13-23.

[4] Laukkanen, M. L., Alfthan, K. and Keinanen, K. (1994) Functional immunoliposomes harboring a biosynthetically lipid-tagged single-chain antibody. Biochemistry 33, 11664-11670.

[5] Kohl, J., Ruker, F., Himmler, G., Razazzi, E. and Katinger, H. (1991) Cloning and expression of an HIV-1 specific single-chain Fv region fused to Escherichia coli alkaline phosphatase. Ann. N. Y. Acad. Sci. 646, 106-114.

[6] Gandecha, A., Owen, M. R., Cockburn, W. and Whitelam, G. C. (1994) Antigen detection using recombinant, bifunctional single-chain Fv fusion proteins synthesised in Escherichia coli. Protein Expr. Purif. 5, 385-390.

[7] Skerra, A. and Pluckthun, A. (1988) Assembly of a functional immunoglobulin Fv fragment in Escherichia coli. Science 240, 1038-1041.

[8] Better, M., Chang, C.P., Robinson, R.R. and Horwit\%, A.H. (1988) Escherichia coli secretion of an active chimeric antibody fragment. Science 240, 1041-1043.

[9] Pluckthun, A. (1991) Antibody engineering: advances from the use of Escherichia coli expression systems. Biotechnology (NY) 9, 545-551.

[10] Field, H., Rees, A.R. and Yarranton, G.T. (1988) A functional recombinant immunoglobulin vairable domain from polypeptides produced in Escherichia coli. Vaccines 88, 29-34.

[11] Bird, R.E., Hardman, K.D.. Jacobson, J.W., Johnson, S., Kaufman, B.M., Lee, S.M., Lee, T., Pope. S.H., Riordan, G.S. and Whitlow. M. (1988) Single-chain antigen-binding proteins Ipublished erratum appears in Science 1989 Apr 28; 244 (4903):409]. Science 242, 423-426.

[12] Cabilly, S. (1989) Growth at sub-optimal temperatures allows the production of functional, antigen-binding Fab fragments in Escherichia coli. Gene 85, 553-557.

[13] Huston, J.S., Levinson. D., Mudgett, H.M.. Tai. M.S., Novotny, J., Margolies, M.N., Ridge, R.J., Bruccoleri, R.E., Haber, E. Crea, R. and Opperman, H. (1988) Protein engineering of antibody binding sites: recovery of specific activity in an anti-digoxin single-chain $\mathrm{Fv}$ analogue produced in Escherichia coli. Proc. Natl. Acad. Sci. USA 85, 5879-5883.

[14] Glockshuber, R., Malia, M.. Pfitzinger. I. and Pluckthun, A. (1990) A comparison of strategies to stabilize immunoglobulin Fv-fragments. Biochemistry 29, 1362-1367. 
[15] Desmyter. A., Transue, T.R., Ghahroudi, M.A., Thi, M.H., Poortmans, F., Hamers, R., Muyldermans, S. and Wyns, L. (1996) Crystal structure of a camel single-domain VH antibody fragment in complex with Iysozyme. Nat. Struct. Biol. 3, 803-811.

[16] Pessi, A.. Bianchi, E., Crameri. A., Venturini, S., Tramontano, A. and Sollazzo, M. (1993) A designed metal-binding protein with a novel fold [see comments]. Naturc 362, 367-369.

[17] Desplancq, D., King, D.J., Lawson, A.D. and Mountain, A. (1994) Multimerization behaviour of single chain Fv variants for the tumour-binding antibody B72.3. Protcin Eng. 7, 1027-1033.

[18] Huston, J.S., Margoies, M.N. and Haber, E. (1996) Antibody binding sites. Adv. Protein Chem. 49, 329-350.

[19] Clackson, T., Hoogenboom, H.R., Griffiths, A.D. and Winter, G. (1991) Making antibody fragments using phage display libraries. Nature 352, 624-628.

[20] Marks, J.D., Hoogenboom, H.R., Bonnert, T.P., McCafferty, J., Griffiths, A.D. and Winter, G. (1991) By-passing immunization: Human antibodies from V-gene libraries displayed on phage. J. Mol. Biol. 222, 581-597.

[21] Hoogenboom, H.R. and Winter, G. (1992) By-passing immunisation. Human antibodies from synthetic repertoires of germline VH gene segments rearranged in vitro. J. Mol. Biol. 227, 381-388.

[22] Padlan, E.A. (1991) A possible procedure for reducing the immunogenicity of antibody variable domains while preserving their ligand-binding properties. Mol. Immunol. 28, 489-498.

[23] Ayala, M., Balint, R.F., Fernandez, d.C.L., Canaan, H.J., Larrick, J.W. and Gavilondo, J.V. (1995) Variable region sequence modulates periplasmic export of a single-chain Fv antibody fragment in Escherichia coli. Biotechniques 18, 835-838.

[24] Krebber, A., Bornhauser, S., Burmester, J., Honegger, A., Willuda, J., Bosshard, H.R. and Pluckthun, A. (1997) Reliable cloning of functional antibody variable domains from hybridomas and spleen cell repertoires employing a reengineered phage display system. J. Immunol. Methods $201,35-55$.

[25] Kipriyanov, S.M., Kupriyanova, O.A., Little, M. and Moldenhauer, G. (1996) Rapid detection of recombinant antibody fragments directed against cell-surface antigens by flow cytometry. J. Immunol. Methods 196, 51-62.

[26] Bradbury, A., Ruberti, F., Werge, T., Amati, V., Di Luzio, A., Gonfloni, S., Hoogenboom, H.R., Piccioli, P., Biocca, S. and Cattaneo. A. (1995) The cloning of hybridoma V regions for their ectopic expression in intracellular and intercellular immunisation. In: Borrebaeck, C.A.K. (Ed.), Antibody Engineering, IRL Press, Oxford, UK., 295-36I.

[27] Brinkmann, U., Reiter, Y., Jung, S.H., Lee, B. and Pastan, I. (1993) A recombinant immunotoxin containing a disulfide-stabilized Fv fragment. Proc. Natl. Acad. Sci. USA 90, 7538-7542. 
[28] Young, N.M.. MacKenzie, C.R., Narang, S.A., Oomen, R.P. and Baenziger, J.E. (1995) Thermal stabilization of a single-chain Fv antibody fragment by introduction of a disulphide bond. FEBS Lett. 377, 135-139.

[29] Reiter, Y., Wright, A.F., Tonge. D.W. and Pastan, I. (1996) Recombinant single-chain and disulfide-stabilized Fv-immunotoxins that cause complete regression of a human colon cancer xenograft in nude mice. Int. J. Cancer 67, 113-123.

[30] Reiter, Y., Brinkmann. U., Jung, S.H., Lee, B., Kasprzyk, P.G., King, C.R. and Pastan, I. (1994) Improved binding and antitumor activity of a recombinant anti-erbB2 immunotoxin by disulfide stabilization of the Fv fragment. J. Biol. Chem. 269, 18327-18331.

[31] Carter, P., Presta, L., Gorman, C.M., Ridgway, J.B., Henner, D., Wong, W.L., Rowland, A.M., Kotts. C., Carver, M.E. and Shepard, H.M. (1992) Humanization of an anti-p185HER2 antibody for human cancer therapy. Proc. Natl. Acad. Sci. USA 89, 4285-4289.

[32] Boulot, G., Eisele, J.L., Bentley, G.A., Bhat, T.N., Ward, E.S., Winter, G. and Poljak. R.J. (1990) Crystallization and preliminary X-ray diffraction study of the bacterially expressed Fv from the monoclonal anti-lysozyme antibody D1.3 and of its complex with the antigen, lysozyme. J. Mol. Biol. 213, 617-619.

[33] Glockshuber, R., Steipe, B., Huber, R. and Pluckthun, A. (1990) Crystallization and preliminary X-ray studies of the VL domain of the antibody McPC603 produced in Escherichia coli. J. Mol. Biol. 213,613-615.

[34] Eigenbrot, C., Randal, M., Presta, L., Carter, P. and Kossiakoff, A.A. (1993) X-ray structures of the antigen-binding domains from three variants of humanized anti-p185HER2 antibody 4D5 and comparison with molecular modeling. J. Mol. Biol. 229, 969-995.

[35] Ysern, X., Fields, B.A., Bhat, T.N., Goldbaum, F.A., Dall'Acqua, W., Schwarz, F.P., Poljak, R.J. and Mariuzza, R.A. (1994) Solvent reartangement in an antigen-antibody interface introduced by site-directed mutagenesis of the antibody combining site. J. Mol. Biol. 238, 496500 .

[36] Marks, J.D., Tristem, M., Karpas, A. and Winter, G. (1991) Oligonucleotide primers for polymerase chain reaction amplification of human immunoglobulin variable genes and design of family-specific oligonucleotide probes. Eur. J. Immunol. 21, 985-991.

[37] Huse, W.D., Sastry, L., Iverson, S.A., Kang, A.S., Alting, M.M., Burton, D.R., Benkovic, S.J. and Lerner, R.A. (1989) Generation of a large combinatorial library of the immunoglobulin repertoire in phage lambda. Science 246, 1275-1281.

[38] Larick. J.W., Danielsson, L., Brenner, C.A., Abrahamson, M., Fry, K.E. and Borrebaeck. C.A. (1989a) Rapid cloning of rearranged immunoglobulin genes from human hybridoma cells using mixed primers and the polymerase chain reaction. Biochem. Biophys. Res. Commun. 160, $1250-1256$. 
[39] Orlandi, R., Gussow, D.H., Jones, P.T. and Winter, G. (1989) Cloning immunoglobulin variable domains for expression by the polymerase chain reaction. Proc. Natl. Acad. Sci. USA 86, 3833-3837.

[40] Ward, E.S., Gussow, D., Griffiths, A.D., Jones, P.T. and Winter, G. (1989) Binding activities of a repertoire of single immunoglobulin variable domains secreted from Escherichia coli [see comments]. Nature 341, 544-546.

[41] Hoogenboom, H.R., Raus, J.C.M. and Volckaert, G. (1990) Cloning and expression of a chimeric antibody directed against the human transferrin receptor. J. Immunol. 144, 33211 33217.

[42] Caroll, W.L., Mendel, E. and Levy, S. (1988) Hybridoma fusion cell lines contain an aberrant kappa transcript. Mol. Immunol. 25, 991-995.

[43] McCafferty, J., Griffiths, A.D., Winter, G. and Chiswell, D.J. (1990) Phage antibodies: filamentous phage displaying antibody variable domains. Nature 348, 552-554.

[44] Smith, G.P. (1985) Filamentous fusion phage: novel expression vectors that display cloned antigens on the virion surface. Science 228, 1315-1317.

[45] Smith, G.P. (1991) Surface presentation of protein epitopes using bacteriophage expression systems. Curr. Opin. Biotechnol. 2, 668-673.

[46] Webster, R. (1996) Biology of the Filamentous Bacteriophage. In: Kay, B., Winter, L. \& McCafferty, J. (Ed.), Phage display of peptides and proteins, Academic Press, San Diego, 1-20.

[47] Zacher, A.N., Stock, C.A., Golden, J.W. and Smith, G.P. (1980) A new filamentous phage cloning vector: fd-tet. Gene 9, 127-140.

[48] Hoogenboom, H.R., Griffiths, A.D., Johnson, K.S., Chiswell, D.J., Hudson, P. and Winter, G. (1991) Multi-subunit proteins on the surface of filamentous phage: methodologies for displaying antibody (Fab) heavy and light chains. Nucleic Acids Res. 19, 4133-4137.

[49] Bass, S., Greene, R. and Wells, J.A. (1990) Hormone phage: an enrichment method for variant proteins with altered binding properties. Proteins 8 , 309-314.

[50] Garrard, L.J., Yang, M., O'Connell, M.P., Kelley, R.F. and Henner, D.J. (1991) Fab assembly and enrichment in a monovalent phage display system. BioTechnology 9, 1373-1377.

[51] Krebber, A., Burmester, J. and Pluckthun, A. (1996) Inclusion of an upstream transcriptional terminator in phage display vectors abolishes background expression of toxic fusions with coat protein $\mathrm{g} 3 \mathrm{p}$. Gene 178, 71 -74.

[52] Kang, A.S., Barbas, C.F., Janda, K.D., Benkovic, S.J. and Lerner, R.A. (1991) Linkage of recognition and replication functions by assembling combinatorial antibody Fab libraries along phage surfaces. Proc. Natl. Acad. Sci. USA 88, 4363-4366.

[53] Marks, J.D., Hoogenboom, H.R., Griffiths. A.D. and Winter, G. (1992) Molecular evolution of proteins on filamentous phage : mimicking the strategy of the immune system. J. Biol. Chem. 267, 16007-16010. 
[54] McCafferty, J. (1996) Phage display: factors affecting panning efficiency. In: Kay, B., Winter, L. \& McCafferty, J. (Ed.), Phage display of peptides and proteins, Academic Press, San Diego, 261-276.

[55] Barbas, C.. Kang. A.S., Lemer, R.A. and Benkovic, S.J. (1991) Assembly of combinatorial antibody libraries on phage surfaces: the gene III site. Proc: Natl. Acad. Sci. USA 88, 7978-7982. [56] Lowman, H.B., Balss, S.H., Simpson, N. and Wells, J.A. (1991) Selecting high-affinity binding proteins by monovalent phage display. Biochem. 30, 10832-10838.

[57] Winter, G., Griffiths, A.D., Hawkins, R.E. and Hoogenboom, H.R. (1994) Making antibody by phage display technology. Ann. Rev. Immunol. 12, 433-455.

[58] Kuwabara, I., Maruyama, H., Mikawa, Y.G., Zuberi, R.I., Liu, F.-T. and Maruyama, I.N. (1997) Efficient epitope mapping by bacteriophage 1 surface display. Nature Biotech. 15, 74-78.

[59] Mikara, Y.G., Maruyama, I.N. and Brenner, S. (1996) Surface display of proteins on bacteriophage lambda heads. J. Mol. Biol. 262, 21-30.

[60] Stemberg, N. and Hoess, R.H. (1995) Display of peptides and proteins on the surface of bacteriophage lambda. Proc. Natl. Acad. Sci. USA 92, 1609-1613.

[61] Maruyama, I.N., Maruyama, H.I. and Brenner, S. (1994) Lambda foo: a lambda phage vector for the expression of foreign proteins. Proc. Natl. Acad. Sci. USA 91, 8273-8277.

[62] Ren. 7.J., Lewis, G.K., Wingfield, P.T., Locke, E.G., Steven, A.C. and Black, L.W. (1996) Phage display of intact domains at high copy number: a system based on SOC, the small outer capsid protein of bacteriophage T4. Protein Sci. 5, 1833-1843.

[6.3] Georgiou, G.. Stathopoulos, C., Daugherty, P.S., Nayak, A.R., Iverson, B. and Curtiss, R. (1997) Display of heterologous proteins on the surface of microorganisms: from the screening of combinatorial libraries to live recombinant vaccines. Nat. Biotechnol. 15, 29-34.

[64] Schreuder. M.P., Mooren, A.T., Toschka, H.Y., Verrips, C.T. and Klis, F.M. (1996) Immobilizing proteins on the surface of yeast cells. Trends Biotechnol. 14, 115-120.

[65] Ager, S., Nilson, B.H., Morling, F.J., Peng, K.W., Cosset, F.L. and Russell, S.J. (1996) Retroviral display of antibody fragments; interdomain spacing strongly influences vector infectivity. Hum. Gene Ther. 10, 2157-2164.

[66] Russell, S.J., Hawkins, R.E. and Winter, G. (1993) Retroviral vectors displaying functional antibody fragments. Nucleic Acids Res. 21, $1081-1085$.

[67] Persson. M.A., Caothien, R.H. and Burton, D.R. (1991) Generation of diverse high-affinity human monoclonal antibodies by repertoire cloning. Proc. Natl. Acad. Sci. USA 88, 2432-2436. [68] Kattleborough, C.A.. Saldanha, J., Ansell, K.H. and Bendig, M.M. (1993) Optimization of primers for cloning libraries of mouse immunoglobulin genes using the polymerase chain reaction. Eur. J. Immunol. 23, 206-211.

[69] Orum. H., Andersen. P.S., Oster, A.. Johansen. L.K., Riise, E., Bjornvad, M., Svendsen, I. and Engburg. J. (1993) Efficient method for constructing comprehensive murine Fab antibody libraries displayed on phage. Nucleic leids Res. 21, 4491-4498. 
[70] L.ang. I.M., Barbas, C.r. and Schleef, R.R. (1996) Recombinant rabbit Fab with binding activity to type-1 plasminogen activator inhibitor derived from a phage-display library against human alpha-granules. Gene 172, 295-298.

[71] Yamanaka. H.I., Inoue, T. and Tkeda, T.O. (1996) Chicken monoclonal antibody isolated by a phage display system. J. Immunol. 157, $1156-1162$.

[72] Davies, E.L., Smith, J.S., Birkett, C.R., Manser, J.M., Anderson, D.D. and Young, J.R. (1995) Selection of specific phage-display antibodies using libraries derived from chicken immunoglobulin genes. J. Immunol. Methods 186, 125-135.

[73] Horton. R.M., Cai, Z.L., Ho, S.N. and Pease, L.R. (1990) Gene splicing by overlap extension: tailor-made genes using the polymerase chain reaction. Biotechniques 8, 528-535.

[74] Alfthan, K., Takkinen, K., Sizmann, D., Seppala, I., T., I., Vanne, L., Keranen, S., Kaartinen, M.. Knowles, J.K. and Teeri, T.T. (1993) Efficient secretion of murine Fab fragments by Escherichia coli is determined by the first constant domain of the heavy chain. Gene 128, 203-209.

[75] Andersen, P.S., Orum, H. and Engberg, J. (1996) One-step cloning of murine Fab gene fragments independent of $\mathrm{IgH}$ isotype for phage display libraries. Biotechniques 20, 340-342.

[76] Griffiths, A.D., Williams, S.C., Hartley, O., Tomlinson, I.M., Waterhouse, P., Crosby, W.L., Kontermann, R., Jones, P.T., Low, N.M., Allison, T.J., Prospero, T.D., Hoogenboom, H.R., Nissim, A., Cox, J.P.L., Harrison, J.L., Zaccolo, M., Gherardi, E. and Winter, G. (1994) Isolation of high affinity human antibodies directly from large synthetic repertoires. EMBO J. 13, 32453260 .

[77] Marks, J.D., Griffiths, A.D., Malmqvist, M., Clackson, T.P., Bye, J.M. and Winter, G. (1992) By-passing immunization: Building high affinity human antibodies by chain shuffling. Bio/Technology 10, 779-783.

[78] Persic, L., Roberts, A., Wilton, J., Cattaneo, A., Bradbury, A. and Hoogenboom, H.R. (1997) An integrated vector system for the eukaryotic expression of antibodies or their fragments after selection from phage display libraries. Gene 187, 9- 18.

[79] Hoogenboom, H.R. (1997) Designing and optimizing library selection strategies for generating high-affinity antibodies. Trends Biotechnol. 15, 62-70.

[80] Malmborg, A.C., Duenas, M., Ohlin, M., Soderlind, E. and Borrebaeck, C.A. (1996) Selection of binders from phage displayed antibody libraries using the BIAcore biosensor. $J$. limmunol. Methods 198, 51-57.

[81] Sanna, P.P., Williamson, R.A., De, L.A., Bloom, F.E. and Burton, D.R. (1995) Directed selection of recombinant human monoclonal antibodies to herpes simplex virus glycoproteins from phage display libraries. Proc. Natl. Acad. Sci. USA 92, 6439-6443.

[82] Ping, T., Tometta, M.A., Ames, R.S., Bankosky, B.C., Griego, S., Silverman, C., Porter, T., Moore, G. and Sweet, R.W. (1996) Isolation of a neutralizing human RSV antibody from a 
dominant, non-neutralizing immune repertuire by epitope-blocked panning. J. Immunol. 157. 772-780.

[83] Ditzel, H.J., Binley, J.M., Moore, J.P., Sodroski, J., Sullivan, N., Sawyer, L.S., Hendry, R.M., Yang, W.P., Barbas, C.F. and Burton, D.R. (1995) Neutralizing recombinant human antibodies to a conformational V2- and CD4-binding site-sensitive epitope of HIV-1 gp120 isolated by using an epitope-masking procedure. J. Immunol. 154, 893-906.

[84] Parsons, H.L., Earnshaw, J.C., Wilton, J., Johnson, K.S., Schueler, P.A., Mahoney, W. and McCafferty, J. (1996) Directing phage selections towards specific epitopes. Protein Eng. 9, 1043-1049.

[85] Bradbury, A., Persic, L., Werge, T., and Cattaneo, A. (1993) Use of living columns to select specific phage antibodies. Biotechnology (NY) 11, 1565-1569.

[86] Hawkins, R.E., Russell, S.J. and Winter, G. (1992) Selection of phage antibodies by binding affinity. Mimicking affinity maturation. J. Mol. Biol. 226, 889-896.

[87] Schier, R., Bye, J., Apell, G., McCall, A., Adams, G.P., Malmqvist, M., Weiner, L.M. and Marks, J.D. (1996) Isolation of high-affinity monomeric human anti-c-erbB-2 single chain Fv using affinity-driven selection. J. Mol. Biol. 255, 28-43.

[88] Roberts, B.L. (1992) Protease inhibitor display M13 phage: selection of high-affinity neutrophil elastase inhibitors. Gene 121, 9-15.

[89] Griffiths, A.D., Malmqvist. M., Marks, J.D., Bye, J.M., Embleton, M.J., McCafferty, J., Baier, M., Holliger, K.P., Gorick, B.D., Hughes, J.N., Hoogenboom, H.R. and Winter, G. (1993) Human anti-self antibodies with high specificity from phage display libraries. EMBO J. 12, 725 734.

[90] Ward, R.L., Clark, M.A., Lees, J. and Hawkins. N.J. (1996) Retrieval of human antibodies from phage-display libraries using enzymatic cleavage. J. Immunol. Methods 189, 73-82.

[91] Meulemans, E.V.. Slobbe, R., Wasterval, P., Ramaekers, F.C. and van Eys, G.J. (1994) Selection of phage-displayed antibodies specific for a cytoskeletal antigen by competitive elution with a monoclonal antibody. J. Mol. Biol. 244, 353-360.

[92] Cili, X. and Garen, A. (1995) Anti-melanoma antibodies from melanoma patients immunised with genetically modified autologus tumor cells : Selection of specific antibodies from single-chain Fv fusion phage libraries. Proc. Natl. Acad. Sci. USA 92, 6537-6541.

[93] Marks, J.D., Ouwehand, W.H., Bye, J.M., Finnem, R., Gorick, B.D., Voak, D., Thorpe, S.J., Hughes, J.N. and Winter, G. (1993) Human antibody fragments specific for human blood group antigens from a phage display library. Biotechnology (NY) 11, $1145-1149$.

[94] Vaughan, T.P., Williams A.W., Pritchard, K., Osboum, J.K., Pope, A.R., J.C., E., McCafferty, J., Hodits, R.A., Wilton, J. and Johnson. K.S. (1996) Human antibody with subnanomolar affinities isolated from a large non-immunized phage display library. Nat. Biotechnol. 14, 309-314. 
[95] de Kruif, J., Terstappen, L., Boel, E. and Logtenberg, T. (1995) Rapid selection of cell subpopulation-specific human monoclonal antibodies from a synthetic phage antibody library. Proc. Natl. Acad. Sci. USA 92, 3938-3942.

[96] Andersen, P.S., Stryhn, A., Hansen, B.E., Fugger, L., Engberg, J. and Buus, S. (1996) A recombinant antibody with the antigen-specific, major histocompatibility complex-restricted specificity of T cells. Proc. Natl. Acad. Sci. USA 93, 1820-1824.

[97] Siegel, D.L. and Silverman, L.E. (1994) Expression and characterization of recombinant anti-Rh(D) antibodies on filamentous phage: a model system for isolating human red blood cell antibodies by repertoire cloning. Blood 83, 2334-2344.

[98] Stausbol-Gron, B., Wind, T., Kjaer, S., Kahns, L., Hansen, N.J., Kristensen, P. and Clark, B.F. (1996) A model phage display subtraction method with potential for analysis of differential gene expression. FEBS Lett. 391, 71-75.

[99] Mandecki, W., Chien, Y.C. and Grihalde, N. (1995) A mathematical Model for biopanning (affinity selection) using peptide libraries on filamentous phage. J. Theor. Biol. 176, 523-530.

[100] Van Ewijk, W., de Kruif, J., Germeraad, W. T., Berendes, P., Ropke. C., Platenburg, P. P. and Logtenberg, T. (1997) Subtractive isolation of phage-displayed single-chain antibodies to thymic stromal cells by using intact thymic fragments. Proc. Natl. Acad. Sci. USA 94, 39033908.

[101] Pasqualini, R. and Ruoslahti, E. (1996) Organ targeting in vivo using phage display peptide libraries. Nature 380, 364-366.

[102] Pereira, S., Van Belle, P., Elder, D., Maruyama, H., Jacob, L., Sivanandham, M., Wallack, M., Siegel, D. and Herlyn, D. (1997) Combinatorial antibodies against human malignant melanoma. Hybridoma 16, 11-16.

[103] Markland, W., Ley, A.C., Lee, S.W. and Ladner, R.C. (1996) Iterative optimization of high-affinity proteases inhibitors using phage display. 1. Plasmin. Biochemistry 35, 8045-8057. [104] Krebber, C., Spada, S., Desplancq, D. and Pluckthun, A. (1995) Co-selection of cognate antibody-antigen pairs by selectively-infective phages. FEBS Lett. 377, 227-231.

[105] Duenas, M. and Borrebaeck, C.A.K. (1994) Clonal selection and amplification of phage displayed antibodies by linking antigen recognition and phage replication. Biotechnology 12 , 999-1002.

[106] Gramatikoff, K., Georgiev, O. and Schaffner, W. (1994) Direct interaction rescue, a novel filamentous phage technique to study protein-protein interactions. Nucleic Acids Res. 22, 5761 5762.

[107] Krebber, C., Spada, S., Desplancq, D., Krebber, A., Ge, L. and Pluckthun, A. (1997) Selectively-infective phage (SIP): a mechanistic dissection of a novel in vivo selection for protein-ligand interactions. J. Mol. Biol. 268, 607-618. 
[108] Zaccolo, M., Griffiths, A.P., Prospero, T.D., Winter, G. and Gherardi. E. (1997) Dimerization of Fab fragments enables ready screening of phage antibodies that affect hepatocyte growth factor/scatter factor activity on target cells. Eur. J. Immunol. 27, 618-623.

[109] Nissim, A., Hoogenboom, H.R., Tomlinson, I.M., Flynn, G., Midgley, C., Lane, D. and Winter, G. (1994) Antibody fragments from a 'single pot' phage display library as immunochemical reagents. EMBO J. 13, 692-698.

[110] Kleymann, G., Ostermeier, C., Heitmann, K., Haase, W. and Michel, H. (1995) Use of antibody fragments (Fv) in immunocytochemistry. J. Histochem. Cytochem. 43, 607-614.

[111] Carnemolla, B., Neri, N., Castellani, P., Veirana, N., Neri, G., Pini, A., Winter, G. and Zardi, L. (1996) High-affinity human recombinant antibodies to the oncofetal angiogenesis marker fibronectin ED-B domain. Int. J. Cancer 68, 397-405.

[112] Cai, X. and Garen, A. (1996) A melanoma-specific VH antibody cloned from a fusion phage library of a vaccinated melanoma patient. Proc. Natl. Acad. Sci. USA 93, 6280-6285.

[113] Lah, M., Goldstraw, A., White, J.F., Dolezal, O., Malby, R. and Hudson, P.J. (1994) Phage surface presentation and secretion of antibody fragments using an adaptable phagemid vector. Hum. Antibodie's Hybridomas 5, 48-56.

[114] Hochuli, E., Bannwarth, W., Döbeli, H., Gentz, R. and Stüber, D. (1988) Genetic approach to facilitate purification of recombinant proteins with a novel metal chelate adsorbent. Bio/Technology 6, 1321-1325.

[115] McGuinness, B.T., Walter, G., FitzGerald, K., Schuler, P., Mahoney, W., Duncan, A.R. and Hoogenboom, H.R. (1996) Phage diabody repertoires for selection of large numbers of bispecific antibody fragments. Nature Biotechnol. 14, 1149-1154.

[116] Persic, L., Righi, M., Roberts, A., Hoogenboom, H.R., Cattaneo, A. and Bradbury, A. (1996) Targeting vectors for intracellular immunisation. Gene 187, 1-8.

[117] Janda, K.D., Lo, L.C., Lo, C.H.L., Sim, M.M., Wang, R., Wong, C.H. and Lerner, R.A. (1997) Chemical selection for catalysis in combinatorial antibody libraries. Science 275, $945-$ 948.

[1 18] Janda, K.D., Lo, C.H., Li, T., Barbas, C.F., Wirsching, P. and Lemer, R.A. (1994) Direct selection for a catilytic mechanism from combinatorial antibody libraries. Proc. Natl. Acad. Sci. USA 91, 2532-2536.

[119] Hart, S.L., Knight, A.M., Harbottle, R.P., Mistry, A., Hunger, H.D., Cutler, D.R., Williamson, R. and Coutelle, C. (1994) Cell binding and internalization by filamentous phage displaying a cyclic Arg-Gly-Asp-containing peptide. J. Biol. Chem. 269, 12468-12474.

[120] Burton, D.R., Barbas, C.F., Persson, M.A.A., Koenig, S., Chanock. R.M. and Lemer, R.A. (1991) A large array of human monoclonal antibodies to type 1 human immunodeficiency virus from combinatorial libraries of asymptomatic individuals. Proc. Natl. Acad. Sci. USA 88, 1013410137. 
[121] Chester, K.A., Begent, R.H., Robson, L., Keep, P., Pedley, R.B., Boden, J.A., Boxer, G., Green, A., Winter, G., Cochet, O. and Hawkins, R. (1995) Phage libraries for generation of clinically useful antibodies. Lanc't 343, 455-461.

[122] Mer $\iota$, D.C.. Dunn, R.J. and Drapeau. P. (1995) Generating a phage display antibody library against an identified neuron. J. Neurosc. Methods 62, 213-219.

[123] Ames, R.S., Tornetta, M.A., Jones, C.S. and Tsui, P. (1994) Isolation of neutralizing antiC5a monoclonal antibodies from a filamentous phage monovalent Fab display library. $J$. Immunol. 153, 910-918.

[124] Popov, S., Hubbard, J.G. and Ward, E.S. (1996) A novel and efficient route for the isolation of antibodies that recognise $T$ cell receptor $V$ alpha(s). Mol. Immunol. 33, 493-502.

[125] Graus, Y.F., de Baets, M.H., Parren, P.W., Berrih-Aknin, S., Wokke, J., van Breda Vriesman, P.J. and Burton, D.R. (1997) Human anti-nicotinic acetylcholine receptor recombinant Fab fragments isolated from thymus-derived phage display libraries from myasthenia gravis patients reflect predominant specificities in serum and block the action of pathogenic serum antibodies. J. Immunol. 158, 1919-1929.

[126] Embleton, M.J., Gorochov, G., Jones, P.T. and Winter, G. (1992) In-cell PCR from mRNA: amplifying and linking the rearranged immunoglobulin heavy and light chain $\mathrm{V}$-genes within single cells. Nucleic Acids Res. 20, 3831-3837.

[127] Ishida, F., Gruel, Y., Brojer, E., Nugent, D.J. and Kunicki, T.J. (1995) Repertoire cloning of a human IgG inhibitor of alpha IIB beta 3 function. The OG idiotype. Mol. Immunol. 32, 61.3622 .

[128] Hawkins, R.E. and Winter, G. (1992a) Cell selection strategies for making antibodies from variable gene libraries: trapping the memory pool. Eur. J. Immunol. 22, 867-870.

[129] Finnern, R., Pedrollo, E., Fisch, I., Wieslander, J., Marks, J.D., Lockwood, C.M. and Ouwehand, W.H. (1997) Human autoimmune anti-proteinase $3 \mathrm{scFv}$ from a phage display library. Clin. Exp. Immunol. 107, 269-281.

[130] Barbas, C.F. and Burton, D.R. (1996) Selection and evolution of high-affinity human antiviral antibodies. Trends Biotechnol. 14, 230-234.

[131] Kettleborough, C.A., Ansell, K.H., Allen, R.W., Rosell-Vives, E., Gussow, D.H. and Bendig, M.M. (1994) Isolation of tumor cell-specific single-chain Fv from immunized mice using phage-antibody libraries and the re-construction of whole antibodies from these antibody fragments. Eur. J. Immunol. 24, 952-958.

[132] Gram, H., Marconi, L., Barbas, C.F., Collet, T.A., Lemer, R.A. and Kang. A.S. (1992) In vitro selection and affinity maturation of antibodies from a naive combinatorial immunoglobulin library. Proc. Natl. Acad. Sci. USA 89, 3576-3580.

[133] Waterhouse, P., Griffiths, A.D., Johnson, K.S. and Winter, G. (1993) Combinatorial infection and in vivo recombination: a strategy for making large phage antibody repertoires. Nucleic Acids Res. 21, 2265-2266. 
[134] Padlan, E.A. (1994) Anatomy of the antibody molecule. Mol. Immunol. 31, 169-217.

[135] Chothia, C. and Lesk, A.M. (1987) Canonical structures for the hypervariable regions of immunoglobulins. J. Mol. Biol. 196, 901-917.

[136] Sanz, I. (1991) Multiple mechanisms participate in the generation of diversity of human $\mathrm{H}$ chain CDR3 regions. J. Immunol. 147, 1720-1729.

[137] Barbas, C.F., Bain, J.D., Hoekstra, D.M. and Lerner, R. (1992) Semisynthetic combinatorial libraries: A chemical solution to the diversity problem. Proc. Natl. Acad. Sci. USA 89, 4457-4461.

[138] de Kruif, J., Boel, E. and Logtenberg, T. (1995) Selection and application of human singlechain FV antibody fragments from a sdemi-synthetic phage antibody library with designed CDR3 regions. J. Mol. Biol. 248, 97-105.

[139] Akamatsu, Y. and Cole, M.S. (1993) Construction of a human Ig combinatorial library from genomic V gene segments and synthetic CDR3 fragments. J. Immunol. 15, 4651-4659.

[140] Garrard, L.J. and Henner, D.J. (1993) Selection of an anti-IGF-1 Fab from a Fab phage library created by mutagenesis of multiple CDR loops. Gene 128, 103-109.

[141] Soderlind, E., Vergeles, M. and Borrebaeck, C.A.K. (1995) Domain libraries : synthetic diversity for de novo desing of antibody V regions. Gene 160, 269-272.

[142] Pere]son, A.S. (1989) Immune network theory. Immunol. Rev. 110, 5-36.

[143] Fisch, I., Kontermann, R.E., Finnern, R., Hartley, O., Soler, G.A., Griffiths, A.D. and Winter, G. (1996) A strategy of exon shuffling for making large peptide repertoires displayed on filamentous bacteriophage. Proc. Natl. Acad. Sci. USA 93, 7761-7766.

[144] Mattheakis, L.C., Bhatt, R.R. and Dower, W.J. (1994) An in vitro polysome display system for identifying ligands from very large peptide libraries. Proc. Natl. Acad. Sci. USA 91, 9022-9026.

[145] Ryabova, L.A., Desplancq, D., Spirin, A.S. and Pluckthun, A. (1997) Functional antibody production using cell-free translation: effects of protein disulfide isomerase and chaperones. Nat. Biotechnol. 15, 79-84.

[146] Nicholls, P.J.. Johnson, V.G., Andrew, S.M., Hoogenboom, H.R., Raus, J.C. and Youle, R.J. (1993) Characterization of single-chain antibody (sFv)-toxin fusion proteins produced in rabbit reticulocyte lysate. J. Biol. Chem. 268, 5302-5308. 


\section{Chapter 1}

\section{EFFECT OF A FR1 MUTATION ON FOLDING OF VH}

Published as

Absolute Conservation of Residue number 6 of Immunoglobulin Heavy

Chain Variable Regions of class IIA is required for correct folding

Hans J.W. de Haard, Bert Kazemier, Arie van der Bent, Peter Oudshoorn, Piet Boender, Bob van Gemen, Jan-Willem Arends and Hennie R. Hoogenboom

Protein Engineering

1998; Volume 11, Nos. 12; pages 1267 - 1276 


\section{Abstract}

While studying the expression of single-chain antibodies (scFv) derived from several murine monoclonal antibodies, we found that residue 6 in Framework region 1 of the heavy chain variable domain plays a crucial role in antibody folding. Binding activity of three murine antibodies with a heavy chain variable region $\left(\mathrm{V}_{\mathrm{H}}\right)$ subgroup IIA was completely lost when at this position the wild type residue glutamine $(Q)$ was substituted by glutamate $(E)$. Increased sensitivity towards trypsin digestion of soluble $s c F v$ suggested that the lack of binding activity was caused by incorrect folding of Q6E mutants. Grafting of the three additional class IA derived FRl residues, based upon the comparison between both classes of VH-sequences, onto the "defect" subgroup IIA sequence, restored antigen binding activity of the Q6E-containing scFv partially. Our results suggest that residue 6 of the heavy chain may be part of a folding nucleus, involving the first two $\beta$ strands of Framework region 1. The evolutionary conservation of either glutamine or glutamate at position 6 in different antibody families may well indicate, that within immunoglobulin VH domains, different family specific folding nuclei have evolved.

\section{Introduction}

The antigen combining site of an antibody consists of the heavy and light chain variable domain $\left(\mathrm{V}_{\mathrm{H}}\right.$ and $\mathrm{V}_{\mathrm{L}}$ ). Each domain contains four framework regions (FR) and three complementarity determining regions (CDR), based on the region's variability in sequence. The 'conserved' framework regions are involved in the formation of two antiparallel $\beta$-sheets typical for the "immunoglobulin fold". The 'hypervariable' CDRs form loops pointing out of the $\beta$ strands and are frequently involved in contacting the antigen.

Although occasionally found to be involved in a direct interaction with antigen (Sheriff et al., 1987; Padlan et al., 1989; Bentley et al., 1990), most FR residues play a passive role, taking part in the $\beta$-sheet scaffold upon which the CDRs are imposed. The numerous studies on CDRgrafted antibodies (Verhoeyen et al., 1989; Riechmann et al., 1988) have provided a clearer insight on the important and sometimes dramatic influence of FR-residues on antigen binding. While grafting CDRs derived from a murine antibody onto a human FR-scaffold to transplant its specificity, it was found frequently necessary to graft murine FR-residues to re-establish the affinity of the original murine antibody (Riechmann et al., 1988). These FR-residues may play an important role in maintaining the structure of one or more of the CDRs (Chothia et al., 1992; Tramontano et al., 1990); indeed, the supportive role of the FR residues located at the edges of the CDRs (dubbed the 'Vernier'-residues) are particularly well studied (Foote and Winter, 1992). The role of the FR residues in determining the affinity of the antibody was recently underlined 
by selection of a number of anti-lysozyme antibody D1.3 FR-residue variants with improved affinity over wild-type (Hawkins et al., 1993).

FR regions, and particular those in $\beta$-turns, have also been found to influence in vivo folding of antibody fragments when produced in bacteria. There are two principle factors that limit expression in bacteria, the first one being related to the folding of the antibody fragment in the periplasmic space, and the other related to the stress imposed by the antibody on the bacteria. The folding efficiency of the antibody fragment may depend on a single amino acid, which determines the kinetic partitioning between folding and aggregation (Knappik and Plückthun, 1995). Some residues may yield folding intermediates with a higher propensity to aggregate, reducing expression yields in vivo; the effect of point mutations on antibody expression therefore is mostly unpredictable.

In this report the unexpectedly drastic effect of mutations in FR1 residues of $V_{H}$ on the folding of single-chain Fv fragments in bacteria is described. During the conversion of threc different mouse antibodies recognizing the human chorionic gonadotropin (hCG) (Birken ' $t$ al., 1990) into bacterially expressed scFv antibodies, variable effects on antigen binding were noted when analyzing variants with mutations in the FRl area of $\mathrm{V}_{\mathrm{H}}$ class IIA containing antibodies. Both heavy chain mutants with wild-type residue $Q$ replaced by $E$ at position 6 showed complete loss of binding, which was restored by introducing other mutations in FR1. We would like to speculate that this FR1 residue may be involved in a family-specific folding nucleus in antibody $V_{\mathrm{H}}$ domains.

\section{Materials and methods}

\subsection{Antibodies and vectors}

Three murine monoclonal antibodies (IgGl, kappa) directed against hCG were used for the conversion into scFvs. These are coded $A B 2 . A B 4$ and $A B 3$; the first two are reactive against $\beta$ core fragment, the latter against B-CTP (nomenclature see Birken et al., 1990). RNA was isolated (Chirgwin et al., 1979) from $5^{*} 10^{7}$ hybridoma cells yielding $600 \mu \mathrm{g}$ RNA. $0.5 \mu \mathrm{g}$ total RNA was transcribed into random primed cDNA, which was used as template for the polymerase chain reaction (PCR).

Phagemid vector pVI, identical to pHENI (Hoogenboom et al., 1991) except having a FLAG-TAG (Hopp et al., 1988), was a gift from P. van Weezenbeek (Organon Int., Oss. The Netherlands). It was constructed in two steps: by PCR assembly (Jayaraman et al., 1989) of two oligonucleotides encoding a new Shine Delgarno-sequence, the pelB-leader and the FLAGepitope, and subsequent coupling of the obtained fragment to a DNA-fragment coding for geneIII (amplified from phage fd-tet (16)) by splicing by overlap extension polymerase chain 
reaction (SOE-PCR) (Horton et al., 1989). The resulting fragment was digested with EcoRI and HindIII and cloned into phagemid vector pSSLL1190 (Pharmacia, Lund, Sweden).

The "T"-vector was prepared from pGEM7zf(+) (Promega, Madison, USA) as was described before (Marchuk et al., 1990).

\subsection{Cloning antibody genes}

For cloning antibody V-regions we have originally used oligonucleotides with restriction sites located within the variable regions, similarly to Orlandi et al., 1989 (primer set 1 in Table I). Amplified DNA was gel purified using the QIAEX system (Qiagen, Hilden, Germany) prior to digestions. $\mathrm{V}_{\mathrm{H}}$ and $\mathrm{V}_{\mathrm{K}}$ encoding PCR fragments were cloned sequentially in pVI using Pst $\mathrm{I}$ or $X h o I$ and $N c o I$ for $V_{\mathrm{H}}$ and $S a l I$ and $B c l$ for $V_{K}$. Electroporations (Dower et al., 1988) were performed in either JM101, a suppressor strain used for phage display when using ambercontaining phagemids (Hoogenboom et al., 1991) or TOP F', a non suppressor for expression of soluble sicFv.

For the determination of the complete wild-type regions $V$-genes were amplified on random primed cDNA with primers located in the constant region and in the leader sequence (primer set 2 Table I). The PCR-fragments were cloned into the T-vector and completely sequenced (sequences are shown in Fig. 1).

To amplify $V$-genes for the construction of phage libraries, we designed primers with restriction sites $S$ fi and NotI located outside the variable coding region as in Clackson et al., 1991 (primer set 3 Table I). DNA fragments encoding $V_{H}, V_{K}$ and the linker were gel purified and combined in a SOE-PCR for assembling the entire scFv encoding gene segment.

The scFv-wt constructs were made in a similar way by amplification with primer set 4 (Table I).

Mutants were constructed by application of SOE PCR with primers encoding the mutation and having an introduced diagnostic (silent) restriction site: the number of cycles was limited to 20. In order to exclude PCR-mistakes the inserts of all clones were completely sequenced with M13 rev-primer, the genelII-primer, linkfwd and linkrev (see Table I).

\section{S.: Panning of phage librurie's}

Rescue of phagemid particles, selection, washing and elution was performed as described (Marks at al.. 1991). Six well tissue culture dishes (Costar, Cambridge MA., USA) were coated with highly purified hCG (Canfield and Morgan et al., 1973) at $3 \mu \mathrm{g} / \mathrm{ml}$ in $50 \mathrm{mM} \mathrm{NaHCO}$ pH9.6 and used for selections. 
A. Heavy chain

\begin{tabular}{|c|c|c|c|c|c|c|c|c|}
\hline \multirow{3}{*}{ sutiboly } & \multirow{3}{*}{ Subgroup } & \multirow[b]{2}{*}{ PRI } & \multirow[b]{2}{*}{ cons } & \multirow[b]{2}{*}{ rez } & \multirow[b]{2}{*}{ CON2 2} & \multirow[b]{2}{*}{ mo } & \\
\hline & & & & & & & $\cos 3$ & red \\
\hline & & $\begin{array}{lll}10 & 20 & 30\end{array}$ & sin. & $40^{\circ}-2$ & $502 \mathrm{~A} \quad 60$ & $70 \quad 102 \mathrm{AnC} \quad 90$ & toOAnCDE 2 & 110 \\
\hline 12 & Ima & 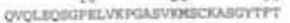 & orvis & 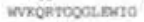 & 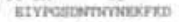 & 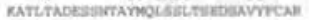 & Worresssemrin & NonetTVTVIS \\
\hline 1) & $\operatorname{rin}$ & $\mathrm{E} \rightarrow 0-\mathrm{TV} \cdot \mathrm{A} M \cdots+\cdots+1 \cdots+\mathrm{D} \cdots \mathrm{h}$ & $5-105 i$ & $\cdots+p_{n} \ldots$ & 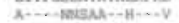 & 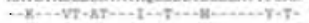 & octrorem vr & $-0-L-\ldots . \cdots$ \\
\hline$\%$ & IIA & 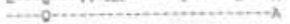 & $\ldots . .$. & n................... & $-7+\cdots+a t-5-8-\cdots+a$ & 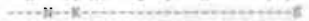 & m-proochl. & 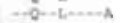 \\
\hline ทекц & ria & 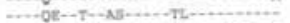 & $-H I-n$ & $\ldots-\mathrm{k}-\mathrm{p}+\ldots \ldots \ldots$ & $n-2-v-a v-\ldots-0--1 e$ & 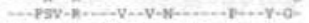 & & \\
\hline$\pi 5-119$ & $\mathrm{In}$ & 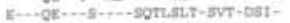 & somen & - THKFT- $-9 K--\mathrm{MM}-$ & $\gamma-5 \gamma-\cos -\gamma+\cdots+F L-5$ & & & \\
\hline OOPC-104E & $\operatorname{IIn}$ & g $\cdots 0$ - & $\cdots$ nox & $\ldots \mathrm{si}-\mathrm{ks}-\ldots$ & $D-5-10000-5-\cdots+0+-G$ & 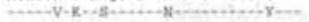 & & \\
\hline lear & $\mathrm{ra}$ & D...-08 - & movan & $-I R-F F-M K-\cdots-M-$ & me $\gamma$-qs-n--psLns & 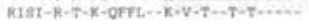 & $\operatorname{cash}$ & $\cdots 0 \cdot 0-5 \cdots E$ \\
\hline
\end{tabular}

\section{B. Light chain}

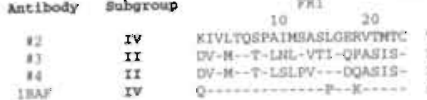

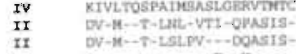

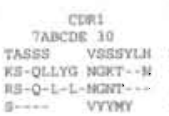

FR2 Ctied
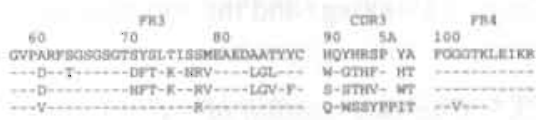

Fig. 1. Deduced amino acid sequences of the variable heavy (A) and light (B) chain regions of anti hCG monoclonal antibodies $\mathrm{AB} 2$ and $\mathrm{AB} 4$. Numbering of the residues and classification of the heavy and light chains was performed according to Kabat (Kabat et al., 1991). In addition the heavy chain V-regions are shown of MBR1 (a class IIA member with glutamate on position 6), TFS-139 (class IA) and MOPC104-E (class IIA). On the bottom lines are the $\mathrm{V}_{\mathrm{H}}$ and the $\mathrm{V}_{\mathrm{L}}$ sequences listed of the anti 2,2,6,6-tetramethyl-1-piperidinyloxy-dinitrophenyl antibody coded IBAF (Leahy $a t$ al., 1988).

Table I. Oligonucleotide primers used for amplification and cloning of variable regions.

\begin{tabular}{|c|c|c|c|}
\hline primerset & primer name & sequence & site \\
\hline \multirow{5}{*}{$\nexists \mathrm{i}$} & VH.FRl & S-AG(G/A) TGC A(G/A) TG CICGAG TCIT/G)G G & who 1 \\
\hline & VH-FR 1 & 5'AGG T(CR)(A/C) A(A/G) C TGCAG $/ \mathrm{CHJ}$ & Pant \\
\hline & VH-FR4 & 5-TGA GGA GACGGT GA CCATGG T CCC TTGGCC & Non 1 \\
\hline & VK-FRI & 5.GACATT CA GTCGAC C CAGTETCCA. & Sarit \\
\hline & VK-FRA & 5-GTI TGA TCA CCA GCT TGG TCCE & Barl. \\
\hline \multirow{5}{*}{22} & VH-leader & S'AGC TGG ATC (T/C)TT CTC TT & \\
\hline & CHI-IgGI & 5-CAG ACC CAG GGG CCA GTG & \\
\hline & VK-leader $(A B 2)$ & 5.ATC AGT GTC ACA GTC ATA (AG)T & \\
\hline & VK-leader(AB $\$)$ & 5.T(TIG)G TG(CT) TCT GGA T(C/T,C CTG & \\
\hline & $\mathrm{CK}$ & 5-TGG ATG GTG GGA AGA TGG AT & \\
\hline \multirow{6}{*}{3} & VH-FRI & 5'-aat a gg ecc agc cec age cggextgg eciG/C) AG(G/A) TCC AGC TG(C/G) AGC AGT CTS G & s*i \\
\hline & VH-FR4 & S-cCT G(AC)G GAG ACG GTG A(C)G)C (A/G)(T/G)A GRE CC & \\
\hline & VK-FR! 1 & 5'-gga tCI (A/G)AA (AGGTT GIG (CIA)TG ACC CAG T/A)CTC & \\
\hline & VK-FR4 & 5'-1at acc g ge gec cgdCCG TIT GAT CAC CAG CTT & Nor1 \\
\hline & VHLINK & S-GGT CAC CGT CTC CTC AgE & \\
\hline & VKLINK & 5. GAG (A/T)CT GCG TCA (GA)CA CAA (T/C)TT (T/C)ag atc $\mathrm{C}$ & \\
\hline \multirow{10}{*}{24} & VH-IRIAB2 & S-3at a ge cec agc cge oc a igg Ce AGG TCC AGC TGE AGC TGG AGC AGT CTG 6 & sei \\
\hline & VH-TR4AB2 & S'-CT' GaG GAG ACG GTG ACC GTG GTC CC & \\
\hline & VK-FRIAB2 & 5'-8Ea IGI AAA ATT GTTCTC ACC CAG TCT CCA & \\
\hline & VK-FRA AB2 4 & S-tat ace g ge gge cge CCG TTI TAT ITE CAG CT GG (C/G)CC & Nati \\
\hline & VHil $r$ NK $A B 2 / 4$ & S-GGI CAC CEI CTC CTC Agg & \\
\hline & VXLINK AB2 & S-AGACTGGOT GAG AAC AAT TTT aga ICC & \\
\hline & VH-FRI AB4 & 5'-aat a ge cee age ceg oc a tgg ceC AGO TTC AGT TGC AGC AGT CTGG & $3=1$ \\
\hline & VH-FR $4 A B A$ & 5'-cCT GCA GAG ACA GTG ACC AGA GTC CC & \\
\hline & VK-FR! ABA & 5-ge tce GAT GTT GTG ATG ACC CAA ACT CCA & \\
\hline & VKLINK A.A4 & 5'-AGT TTE GGT CATCAC AAC ATC aga toc & \\
\hline \multirow{3}{*}{ sequenciese } & geneIII & 5-tga ait uc tgi atg agg & \\
\hline & linkfwd & 5'-ctc gigg cgg tge tgg gtc & \\
\hline & tinkrey & 5. gac cca cca ccg cec gag & \\
\hline
\end{tabular}




\subsection{Refolding of scFvs}

The cells from a $50 \mathrm{ml}$ culture were applied for refolding by resuspending in $8 \mathrm{~mJ} 8 \mathrm{M}$ urea solution (in PBS). After sonication the mixture was rotated head over head for $30 \mathrm{~min}$ and insoluble material was removed (centrifugation for $30 \mathrm{~min}$ at $13000 \mathrm{xg}$ ). The supernatant was dialysed against PBS with 4 buffer changes. Insoluble protein was removed by centrifugation (30 $\min$ at $13000 \mathrm{xg}$ ) and the supernatant was passed through a $0.2 \mu \mathrm{m}$ filter.

\subsection{Characterisation of $s c F v s$}

Large scale productions were performed as described before (Marks et al., 1992) in $50 \mathrm{ml}$ medium. After induction with IPTG growth was continued for $4 \mathrm{~h}$ at $30^{\circ} \mathrm{C}$. Cells were pelleted and used for the preparation of periplasmic fraction using the borate buffered saline (BBS) shock procedure (Skerra and Plückthun, 1991).

Production of individual clones was monitored on $12 \%$ polyacrylamide gels, which were blotted on nitrocellulose. ScFv was detected with anti-FLAG M2 (Kodak IBI, New Haven, USA) $1 / 4000$ diluted in PBST, as conjugate we used anti-mouse peroxidase (DAKO, Glostrup, Denmark) 1/4000 diluted in PBS. The conjugate was detected with the ECL-detection kit (Amersham, Buckinghamshire, UK).

For ELISA microtiterplates were coated with 4 or $10 \mu \mathrm{g} / \mathrm{ml}$ purified hCG in $50 \mathrm{mM}$ $\mathrm{NaHCO}_{3} \mathrm{pH} 9.6$ (overnight at roomtemperature). The wells were blocked with BSA $(0.2 \%(w / v)$ in $0.1 \mathrm{M}$ Tris pH 7.4. $30 \mathrm{mM} \mathrm{KI}$ ). The periplasmic tractions or refolded scFvs were diluted in sample dilution buffer (PBS containing 20\% (v/v) normal goat serum and 1\% Triton X-100). Bound scFv was detected with anti-FLAG and anti mouse peroxidase conjugate. Staining was performed with tetramethylbenzidine (TMB) and ureaperoxide as substrate.

The amount of scFv was determined on Western blot with a model GS-700 Imaging Densitonter (BIORAD, Hercules, CA, USA) using a standard curve of purified ScFv.

For kinetic analysis on the BIAcore, CM-chips (Pharmacia, Lund, Sweden) were applied for covalent coupling of hCG or anti FLAG antibody M2. Experiments were performed with a high density coat of hCG (4369 response units (RU)) or anti-FLAG (9567 RU) (see Figure 3).

\subsection{Trypsin digestion}

The periplasmic fractions prepared from clones $\mathrm{AB} 2-\mathrm{WT}$ and $\mathrm{AB} 4-\mathrm{Q} 6 \mathrm{E}$ were combined (containing approx. $50 \mathrm{ng}$ of each $\mathrm{scFv}$ ) in a single digestion reaction containing $100 \mathrm{mM}$ Tris$\mathrm{HCl} \mathrm{pH} 8.0$ and $10 \mathrm{mM} \mathrm{MgCl}_{2}$ and 200 or $40 \mu \mathrm{g} / \mathrm{ml} 2.5 \%$ trypsin (ICN Biomedicals Inc, Costa Mesa, CA, USA). Altematively, a mixture was made of the extracts from clones AB4-WT and AB2-Q6E. After 2 hours incubation at $16^{\circ} \mathrm{C}$ the digestion was stopped by adding Laemmli buffer. The samples were analysed on Western blot. 


\section{Results}

\section{$4.1 V_{H}-F R I$ mutations affecting antigen binding}

Two different murine anti-hCG monoclonal antibodies, binding different regions on the $\beta$ core of hCG and coded $\mathrm{AB} 2$ and $\mathrm{AB} 4$, were converted into single-chain Fv's (scFvs) and expressed by secretion in bacteria. Initially the $\mathrm{scFv}$ cassettes were amplified using oligonucleotides with restriction sites located within FR1 and FR4 (as in Ward et al., 1989), but the resulting clones, $\mathrm{AB} 2 / 4-\mathrm{Pst}$ and $\mathrm{AB} 2 / 4-\mathrm{Xho}$ in Table II, were surprisingly inactive. In an alternative approach small phage display libraries were made, using degenerate oligonucleotide primers with cloning sites based outside the variable regions (as in Clackson et al., 1991). After selection on hCG, several functional scFvs were found (AB2/4-PHI and AB2/4-PH2 in Table

Table II. Antigen binding characteristics and FR1 sequences of the recombinant scFvs.

\begin{tabular}{|c|c|c|c|c|c|c|c|}
\hline \multirow[t]{2}{*}{$A B$} & \multirow[t]{2}{*}{ Clone } & \multirow{2}{*}{$\begin{array}{l}\text { sequence a }^{a} \\
\text { ER1-VH }\end{array}$} & \multirow{2}{*}{$\begin{array}{l}\text { Prod }{ }^{b} \\
\mu g / L\end{array}$} & \multicolumn{2}{|c|}{ ELISA $^{C}$} & \multicolumn{2}{|c|}{ BIA $^{d}$ ahcG } \\
\hline & & & & $\mathrm{OD}_{450}$ & $\mu g / L$ & $\mathrm{RU}$ & $\mu g / m 1$ \\
\hline & & 12345678 & & & & & \\
\hline \multirow[t]{9}{*}{$A B 2$} & WT & QVQLEQSG & $355=42$ & $0.37=0.04$ & 178 & 320 & 1.8 \\
\hline & PH1 & $\mathrm{E}-\cdots \mathrm{Q}-\cdots$ & $618=17$ & $1.79=0.03$ & 309 & 695 & 3.1 \\
\hline & PH.2 & $\ldots \ldots$ & $630=11$ & $1.69=0.20$ & 315 & 812 & 3.2 \\
\hline & $\mathrm{HF} 2$ & $\ldots$ & $256=59$ & $1.45=0.10$ & 128 & 870 & 5.1 \\
\hline & HF 4 & $\cdots-Q \cdots$ & $238+19$ & $1.83=0.05$ & 119 & 790 & 4.8 \\
\hline & Pst & $\ldots$ HE- & $75=1$ & $0.053=0.005$ & 375 & -14 & 1.5 \\
\hline & Xho & $\cdots$ LE- & $127 \pm 3$ & $0.070 \pm 0.006$ & 635 & -7 & 2.5 \\
\hline & Q6E & - E- & $25 \pm 2$ & $0.054=0.003$ & 113 & -12 & 0.5 \\
\hline & & 12345678 & & & & & \\
\hline \multirow[t]{8}{*}{ AB4 } & WT & QVQLQQSG & $462=14$ & $2.22=0.06$ & 116 & 1438 & 2.3 \\
\hline & $\mathrm{PH} 1$ & $\ldots \ldots$ & $153=30$ & $2.45=0.16$ & 38 & 683 & 0.8 \\
\hline & PH2 & $\mathrm{NI}--\mathrm{E} \cdots$ & $140=35$ & $1.71=0.25$ & 35 & 615 & 0.7 \\
\hline & HF2 & $\cdots-E-\cdots$ & $212=17$ & $2.61=0.01$ & 53 & 1222 & 1.1 \\
\hline & HF 4 & $\ldots \ldots$ & $399 \pm 59$ & $1.64=0.31$ & 100 & 456 & 2.0 \\
\hline & Pst & $\ldots-\mathrm{E}-$ & $3.2 \pm 0.3$ & $0.053=0.002$ & 16 & -13 & 0.06 \\
\hline & Q6E & $\cdots-E-$ & $9.3 \times 1.6$ & $0.067=0.006$ & 47 & 11 & 0.9 \\
\hline & & 12345678 & & & & & \\
\hline \multirow[t]{3}{*}{$\mathrm{AB} 3$} & WT & EVQLQQSG & & & & & \\
\hline & PH1 & Q - E - & $30^{e}$ & $2.75=0.01$ & 75 & 382 & 0.2 \\
\hline & Q6E & QWE- & $0.2^{\circ}$ & $0.065=0.006$ & 1 & -8 & 0.01 \\
\hline
\end{tabular}

\footnotetext{
${ }^{a}$ The clones AB2-PH2 and -HF2 and AB4-PH1 and - HF4 and AB4-Pst and -Q6E differ from each other by the other primer encoded FRs.

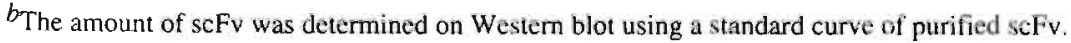

${ }^{c}$ For ELISA plates were coated with $4 \mu \mathrm{g} / \mathrm{ml} \mathrm{hCG}$; background signals were in the $0.05-0.08$ range.

${ }^{d}$ BIAcore experiments were performed with a high density hCG surface (4369 RU) (see Figure 3 ). The hCGreactivity was determined at time point $t=400 \mathrm{~s}$.

$e_{\text {Single observation. }}$
} 
II), which after sequencing were found to differ from the first batch of scFvs only in the oligonucleotide-encoded regions of $\mathrm{V}_{\mathrm{H}^{-}}$and $\mathrm{V}_{\mathrm{L}}$-FRs. Constructs containing the complete wild type sequence (AB2/4-WT in Table II), based on sequence analysis of the V-genes including the Ig-leader (see Materials and methods), and cloned after amplification with wild type FR-specific primers, also expressed functional scFv fragments. Thus the non binding of the first scFvs, $\mathrm{AB} 2 / 4-\mathrm{Pst}$ and $\mathrm{AB} 2 / 4-\mathrm{Xho}$, must have been caused by one or more mutations in one of the FRs of $\mathrm{V}_{\mathrm{H}}$ or $\mathrm{V}_{\mathrm{L}}$, introduced by the oligonucleotides used for the amplification.

The fact that this happened in two murine antibodies which recognise different epitopes on the same antigen was sufficiently intriguing to prompt us to search for the mutation responsible for loss of antigen binding. We only changed the $V_{H}-F R 1$ region of clones $A B 2 / 4-P S t$ and $\mathrm{AB} 2 / 4-\mathrm{Xho}$ into wild type. Functionality was now regained as indicated by ELISA (AB2-HF2 and $\mathrm{AB} 4-\mathrm{HF} 4$ in Table II). Alignment of the $\mathrm{V}_{\mathrm{H}}-\mathrm{FRl}$ encoded primer sequences of the functional and non functional clones as shown in Table II revealed two candidate residues for causing the problem: the residues at positions 5 and 6 . Mutants of $A B 2$ made with the $V_{H^{-}} F R 1$ sequence of $A B 4$ (coded AB2-HF4) and of AB4 with the $V_{H}$ FRI sequence of AB2 (coded AB4$\mathrm{HF2}$ ) were functional, leading us to exclude residue 5 . The involvement of the only remaining residue, $\mathrm{E}$ at position 6 , was confirmed by the generation of antigen binding deficient scFvs with wild type residue glutamine of position 6 substituted by glutamate (clones coded AB2/4-Q6E in Table II). These mutants were indeed inactive in ELISA.

A third hCG antibody, coded AB3. was also non-functional when the wild type residue number 6 glutamine was mutated into glutamate (Table II).

\subsection{Analysis of hCG reactivity in ELISA and BIAcore}

We first analysed the effect of the antigen-binding loss mutations on the total production of the scFvs. The Q6E mutant scFvs were tested using periplasmic preparations and compared with the other scFvs, for binding in ELISA (Table II), and for production by Western blots (Figure 2).

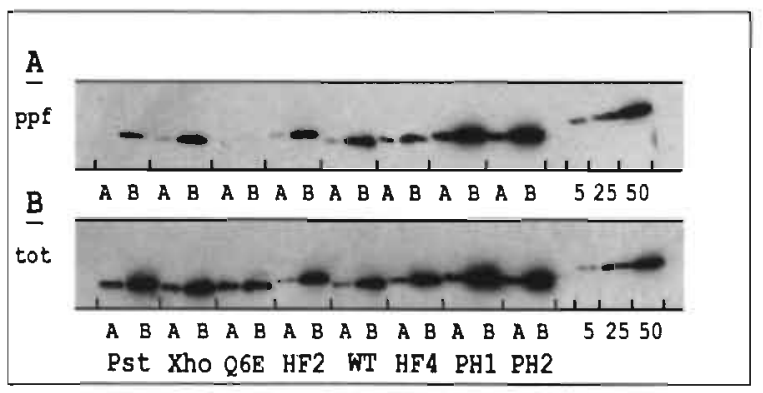

Fig. 2. Western blot detection of the scFvs produced by the different $A B 2$ clones with the anti-FLAG-antibody M2.

Pancl A : periplasmic fractions, lane $A 0.5 \mu 1$ $(0.14 \%$ of a $35 \mathrm{ml}$ culture) and lane B $2.5 \mu \mathrm{l}$ $(0.71 \%)$ periplasmic fraction; Panel $B$ : total cell protein (the cells from $0.5 \mathrm{ml}$ eulture were boiled in $200 \mu \mathrm{l}$ Laemmli sample buffer): lane A $1 \mu \mathrm{l}(0.007 \%$ of a $35 \mathrm{ml}$ culture) and lane B $5 \mu 1(0.035 \%)$ of the sample. The obtained signals were compared with three differen amounts of affinity purified scFv $(5,25.50 \mathrm{ng}$ : right hand lanes). 
The concentration of $\mathrm{scFv}$ was determined by comparison of the measured sample signal with the signal of a range of standards (AB4-PH2 purified on an antigen column and quantified with $\mathrm{OD}_{280}$ ) present on the same blot.

First of all, there were no real differences between the total production levels, i.e. the sum of insoluble and soluble scFv (Figure 2, panel B), of non-functional (AB2-Pst, AB2-Xho, AB2$\mathrm{Q} 6 \mathrm{E})$ and functional clones (AB2-WT, $\mathrm{AB} 2-\mathrm{HF} 2 / 4, \mathrm{AB} 2-\mathrm{PH} 1 / 2)$, thereby excluding variations in mRNA levels or translation. However, examination of the soluble scFv, as was found in the periplasmic fractions, revealed that the E6-containing mutants in general produce lower amounts (Figure 2, panel A; Table 11).

A possibility is that the mutation influences the affinity of the antibody, and that binding would be reduced to a level not detectable by ELISA; therefore the amount of soluble scliv usced in the ELISA tests was scored. All non binding scFvs gave no-binding, background level signals at scFv concentrations as high as $115-635 \mathrm{ng} / \mathrm{ml}$ (AB2) and 16-260 $\mathrm{ng} / \mathrm{ml}$ (AB4). The enormous difference in ELISA suggests that these scFvs have lost all binding completely; for example active clone $\mathrm{AB} 2-\mathrm{HF} 2$ gave a signal of 29 times background level binding at a concentration of $128 \mathrm{ng} / \mathrm{ml}$, contrasting inactive clone $\mathrm{AB} 2-\mathrm{Xho}$, where no binding was detected even at $\mathrm{scFv}$ concentrations of $635 \mathrm{ng} / \mathrm{ml}$.

To test whether the kinetics of binding had been modified by the Q6E mutation, for example a dramatic shift in the off-rate had been induced, the scFvs were tested in BlAcore using hCG immobilized on the Sensorchip (Figure 3). The ELISA results (Table II) were confirmed in terms of reactivity against antigen: all $\mathrm{V}_{\mathrm{H}}$-FRl variants which scored negative in ELISA, did not react with hCG in BIAcore either at all. The measurement should detect any residual reactivity of the scFvs produced by the Q6E variants. Again the difference is striking: of the reactive clone AB2WT the surface captures $320 \mathrm{RU}$, when scFv was injected at a concentration of $1.8 \mu \mathrm{g} / \mathrm{ml}$, while AB2-Xho scores only $-7 \mathrm{RU}$, even at scFv concentrations of $2.5 \mu \mathrm{g} / \mathrm{ml}$. To exclude that the immobilisation of antigen on the sensor chip surface itself had differentially altered the epitope on hCG and therefore its affinity and kinetics of binding by the scFv variants, the binding analysis was carried out also with indirectly immobilised scFv fragments. Anti-FLAG antibody $\mathrm{M} 2$ was coated and used to capture scFv fragments in periplasmic extracts as is shown Figure 3B. Upon injection of $\mathrm{hCG}$, a significant rise in signal was obtained for clone AB2-WT, but for the inactive clone $A B 2-X$ ho the signal returns to its level reached before injection; this while the amount of captured scFv of clone AB2-WT is lower than for AB2-Xho. In this assay, nonbinding was confirmed for all ELISA-negative clones (results not shown). Thus the Q6E mutation causes complete loss of antigen binding in two unrelated antibodies binding to different epitopes of hCG, hinting towards a common cause beyond effect on antigen binding.

In order to exclude problems caused by the single chain format itself, we constructed Fabproducing clones (Hoogenboom et al.. 1991; Carter et al. 1992) from AB4, with or without the Q6E mutation. The wild type Fab reacted in BIAcore and ELISA while the Q6E mutant fatiled to 
do so (results not shown), proving the effect of the mutation on antibody expression is format independent. Thus the reactivity problems of these antibodies are related to the primary sequence of their $\mathrm{V}_{\mathrm{H}}$ domains.

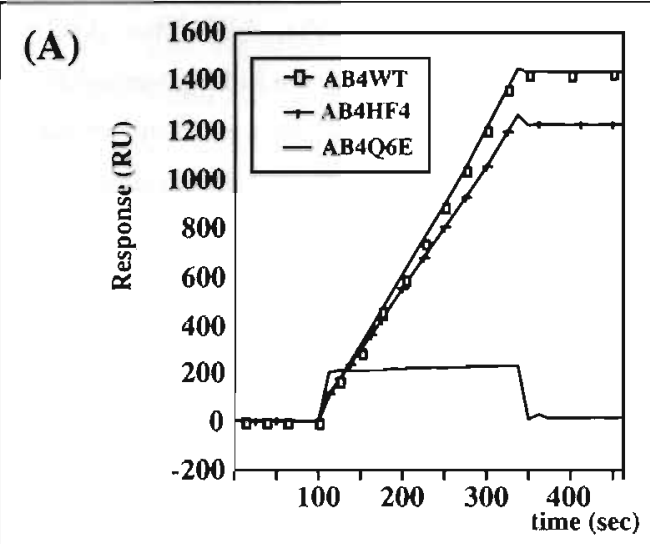

(B)

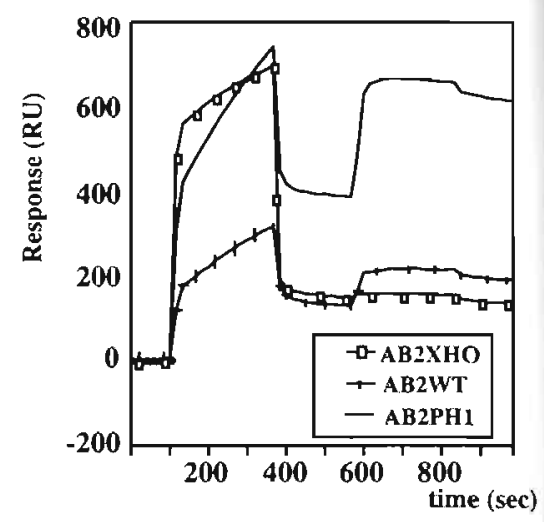

(C)

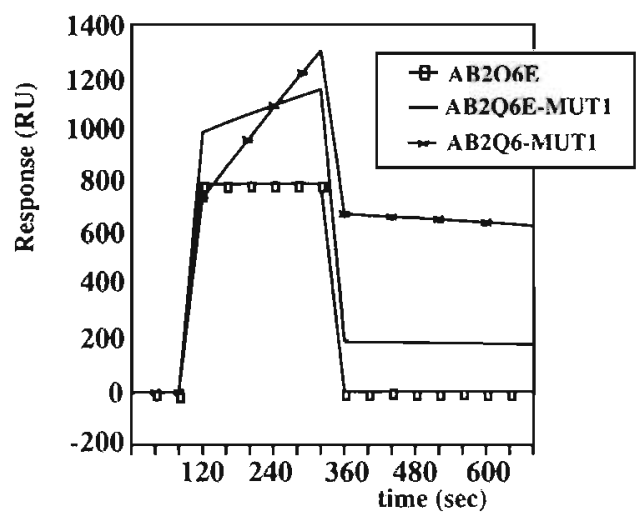

Fig. 3. Binding analysis of the reactivity of the scFvs against hCG using surface plasmon resonance (BIAcore). (A) Overlay plot of binding profiles of three AB4 derivatives on a high density coated hCG sensor chip (4369 RU hCG immobilised). At $t=100 \mathrm{~s}$ the periplasmic fraction was injected; the linear relation between response and time indicates that the association is diffusion limited (Jonsson et al., 1991). At $t=340 \mathrm{~s}$ the surface was washed with Hepes-buffered saline (HBS) buffer. (B) Overlay plot of three AB2 derivatives. ScFv was injected at $t=100 \mathrm{~s}$ on a high density coated anti FLAG M2 chip ( $9667 \mathrm{RU}$ ). After washing, which started at $\mathrm{t}=360 \mathrm{~s}$, antigen binding was analysed by injecting hCG (concentration of $15 \mu \mathrm{g} / \mathrm{ml}$ ) at $\mathrm{t}=570 \mathrm{~s}$. The amount of captured $\mathrm{hCG}$ can be deduced during the following washing cycle starting at $t=850 \mathrm{~s}$. The amount of captured $\mathrm{hCG}$ is the difference between the timepoint before hCG-injection $(\mathrm{t}=550 \mathrm{~s}$ ) and at the start of the second buffer wash ( $t=860 \mathrm{~s}$ ). (C) Overlay plot of three $A B 2$ derivatives injected at $t=100 \mathrm{~s}$ on a high density coated hCG-chip ( $4369 \mathrm{RU}$ hCG immobilised). At $\mathrm{t}=80 \mathrm{~s}$ periplasmic fraction ( $1: 1$ diluted with HBS) was injected; on $i=360 \mathrm{~s}$ the dissociation phase started by washing the surface with HBS-buffer. 
Often antibody fragments produced in bacteria mis-fold, and active materials may be retrieved by in vitro refolding. Refolding as described previously (Glockshuber et al., 1992) of the aggregated scFv resident in the spheroplasts of the Q6E scFvs improved the yield of soluble scFv protein, but did not lead to any antigen binding product. The efficiency of the refolding procedure was shown by applying it on aggregates of functional clones. High yields of refolded antigen binding ScFrs were obtained as was measured by ELISA, BIAcore and by quantitative isolation of ScFv on antigen columns (results not shown). Thus the Q6E mutation must affect both the in vivo and in vitro folding dramatically, in such way that no antigen-binding material can form.

In conclusion we can state, that all attempts to detect antigen binding of the E6-mutant scFvs or Fabs in ELISA or BIAcore failed, in spite of the fact that the amount of soluble fragment should be sufficient for yielding positive signals. Even increasing the amount of soluble scFv by refolding did not improve the test results. These findings indicate that the mutation causes a completc loss of binding allinity.

\subsection{Analysis of conformation by trypsin digestion.}

The integrity of Q6E and wild type of $\mathrm{AB} 2$ and $\mathrm{AB} 4$ was examined by trypsin digestion of the soluble material, as done before with AB-fragments (Glockshuber et al., 1990) or with $\beta$ lactamase (Minsky et al., 1986). The wild type and the Q6E mutant scFv of the two different $\mathrm{ABs}$ were mixed in equal amounts. After incubation with trypsin both types of $\mathrm{AB}$ fragments were separated by electrophoresis and detected on Western blot with anti FLAG AB (Figure 4).

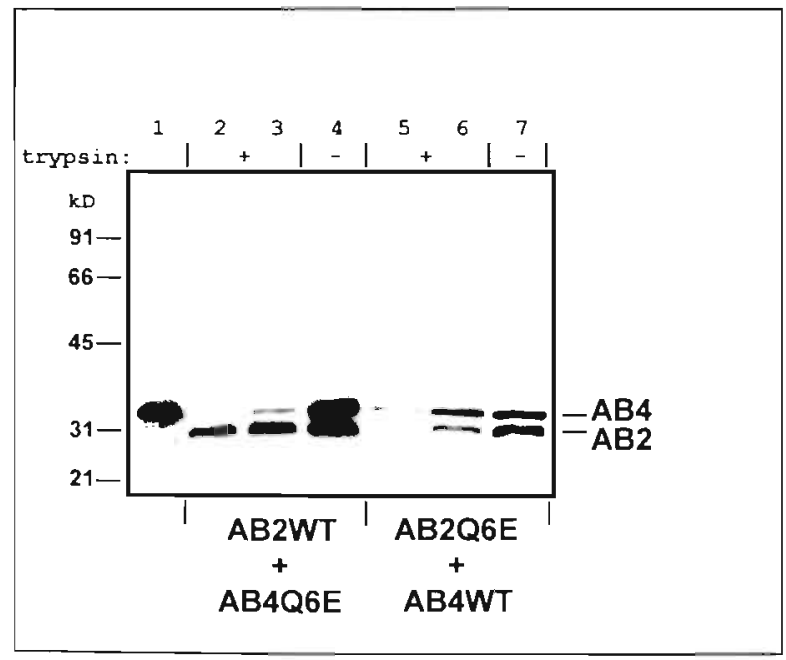

Fig. 4. Trypsin aceessibility experiment on wild-type and Q6E mutant $5 c F v$ fragments derived from AB2 and AB4. Periplasmic extracts were mixed in such a way that an equat amount of each $s c F v$ was included in the digestion reaction. Trypsin addition is indicated with + and -: the samples of lanes 2 and 5 were incubated with $0.2 \mathrm{mg} / \mathrm{ml}$ trypsin. those from lanes 3 and 6 with $40 \mathrm{mg} / \mathrm{ml}$. Lanes 4 and 7 are controls (no digestion). The reactions were stopped by adding SDSPAGE loading buffer and analysed on $15 \%$ polyacrylamide gels to separate the $\mathrm{AB} 2$ and $\mathrm{AB} 4$ scFvs. After blotting. $\mathrm{scFv}$ was detected with anti-FLAG. As a positive control AB4-WT is shown in lane 1

The two Q6E mutants are more rapidly degraded by trypsin than the wild-type proteins. Thus the Q6E mutants are much more protease sensitive, indicating misfolding, while the functional scFvs 
are relatively well protected against protease due to their conrectly formed immunoglobulin fold. In addition the amount of soluble scFv produced by the non functional clones is reduced when compared to the functional versions (and sometimes quite dramatically, e.g. the Q6E mutants of AB2 and AB4 in Table II), however, soluble fragments are still produced. Since unfolded material would be normally present as aggregate, the protease sensitive Q6E protein found in the soluble periplasmic fraction must be misfolded, explaining the complete absence of binding affinity, but it is not aggregated. Figure 5A shows a hypothetical model for folding of the functional scFvs, in which intermediary A can not be detected since it is rapidly transformed into the native (active) state or in the aggregated state. In the Q6E mutant the folding pathway to this end product is blocked, and, besides a lot of aggregated protein, a protease sensitive, nonbinding protein is formed (Figure $5 \mathrm{~B}$ ).

\section{A. Wild-type Q6 containing IlA antibody}

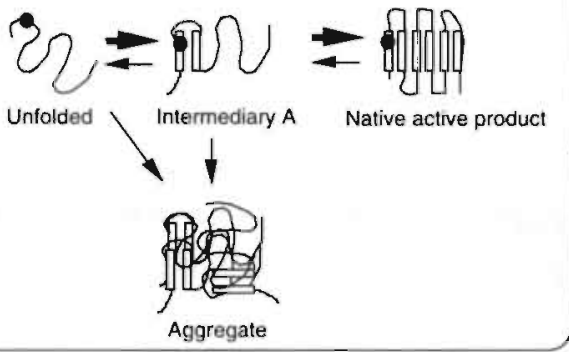

B. Q6E mutants of IIA antibodies

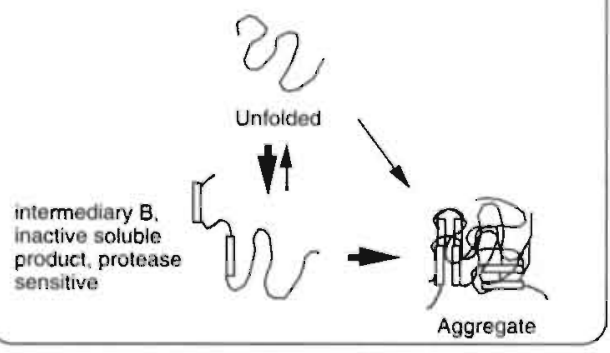

C. Q6E mutants with additional mutations in FR1

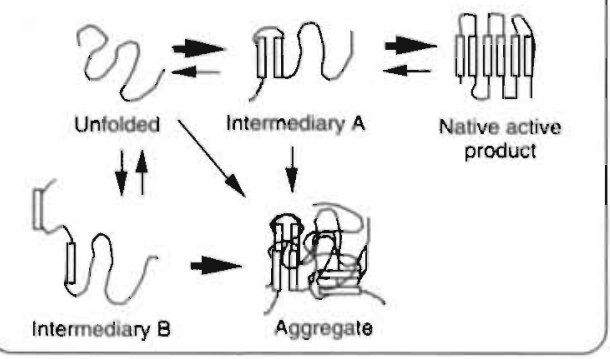

Fig. 5. Hypothetical model of the different folding pathways that are followed by the VH domains of the various antibodies studied.

(A) In antibodies with IIA-VH sequences with the wild-type Q6 the formation of the eorrect folding interinediary $\mathrm{A}$ is promoted. Although part of the protein is lost due to aggregation, the majority can be found as soluble and antigen binding molecules. (B) Mutation of $Q$ to $E$ at position 6 prevents formation of folding intermediary $A$ and leads to an alternative intermediary. B, with enhanced propensity for protease eleavage and aggregation. The intermediate may be found as a soluble protein, but cannot bind antigen.

(C) Additional mutations in FR1 restore the stability of folding intermediary $A$, and thus production of correctly folded antigen binding antibodies. Residue 6 is indicated with a small black (Q) or grey (E) circle: compensating mutations in FRI are indicated with an open white circle (in C). Putative $\beta$-strands are indicaled. 


\subsection{Compensating mutations for the $E(6)$ mutant in $V_{H}$ of $A B 2$ and $A B 4$}

Analysis of the structures did not reveal any eye catching differences between related antibodies with either $\mathrm{Q}$ or $\mathrm{E}$ at position 6 in the immediate vicinity. In the murine antibody directed against 2,2,6,6-tetramethyl-1-piperidinyloxy-dinitrophenyl (coded IBAF; Leahy et al., 1988), of which the structure has been solved by X-ray diffraction, residue 6 (E) is positioned between and may interact with residues from $\beta$-strands $\mathrm{B}, \mathrm{F}$ and $\mathrm{G}$ (Figure $6 \mathrm{~A}$ and $6 \mathrm{~B}$ ). First. aspartic acid at position 96 (Kabat numbering (Kabat et al., 1991)) was converted into asparagine in $\mathrm{AB} 2$; secondly lysine at position 23 was substituted by alanine. Neither mutation affected antigen binding in the wild type construct (Q6); however, when introduced in the mutant (E6) version, they did not result in the production of functional scFv.

Since this approach guided by the putative interaction of residue 6 with other variable domain residues did not offer any other hints, we changed our approach by looking at sequences from antibodies which naturally harbour glutamate at position 6. The studied anti-hCG antibodies possess variable heavy chain regions belonging to subgroup IIA (Kabat el al., 1991). $97 \%$ of the known IIA antibodies have glutamine on position 6 , indicating that it is highly conserved. In contrast, subgroup IA antibodies use mainly glutamate at position $6(99 \%)$. This family-dependent conservation is remarkably well visible in human $\mathrm{V}_{\mathrm{H}}$ genes also (Tomlinson et al., 1992). We aligned the sequences of the V-regions of the hCG antibodies, together with a IIA antibody having a glutamate residue, which is coded MBR1 (Orlandi et al., 1989), and TFS-129. a $\mathrm{V}_{\mathrm{H}}$-Class IA antibody (Figure 1). The main differences within the aminoterminal part are on position 13 (lysine present in the anti hCG antibodies and in IIA, versus serine in MBR1), 19 (lysine in IIA versus threonine in MBRI or serine in IA) and 20 (methionine or isoleucine in anti hCG's versus leucine in MBR1 or IA). We changed all three residues within AB2 into their counterparts of MBRI (K13S; K19T; M20L) again in the wild type construct AB2-WT ('Q6'), and in the mutant $\mathrm{AB} 2-\mathrm{Q} 6 \mathrm{E}$ ('E6'), yielding 'MUT1' versions (Table III). The amount of soluble scFv present in the periplasmic fractions was determined on Western blot, while the hCG reactivity was tested in BIAcore and ELISA (Table II). As shown in BIAcore (Figure 3C) and ELISA, antigen reactivity was obtained with the periplasmic fraction of the E6 triple mutant (containing the three mutations located within the FRI-region). In hCG ELISA a signal of 6 times background was obtained at a concentration of $1650 \mathrm{ng} / \mathrm{ml}$ and of 1.5 times background at dilutions of $165 \mathrm{ng} / \mathrm{ml}$. For comparison: the Q6 triple mutant gave signals of 23 times background measured at a concentration of $187 \mathrm{ng} / \mathrm{ml}$, indicating that this antibody fragment has a higher affinity or, most likely, that a smaller fraction of the 'E6 triple mutant' consists of active protein. A higher signal was found also in BIAcurc for the Q6 triple mutant compared to the E6 triple mutant (respectively 679 versus 195 RUs; see Table III). 


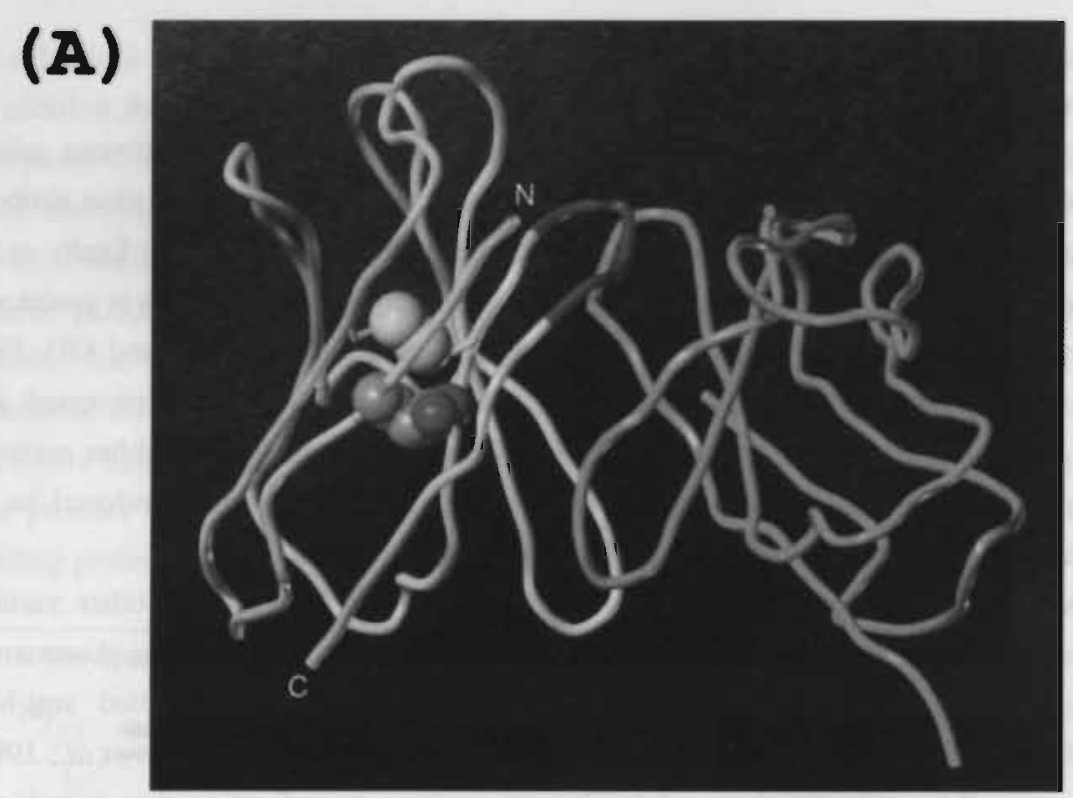

\section{(B)}

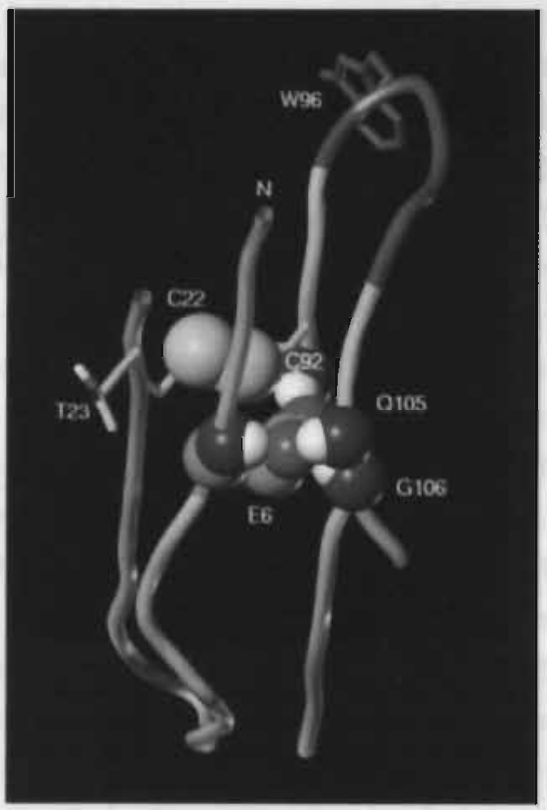

(C)

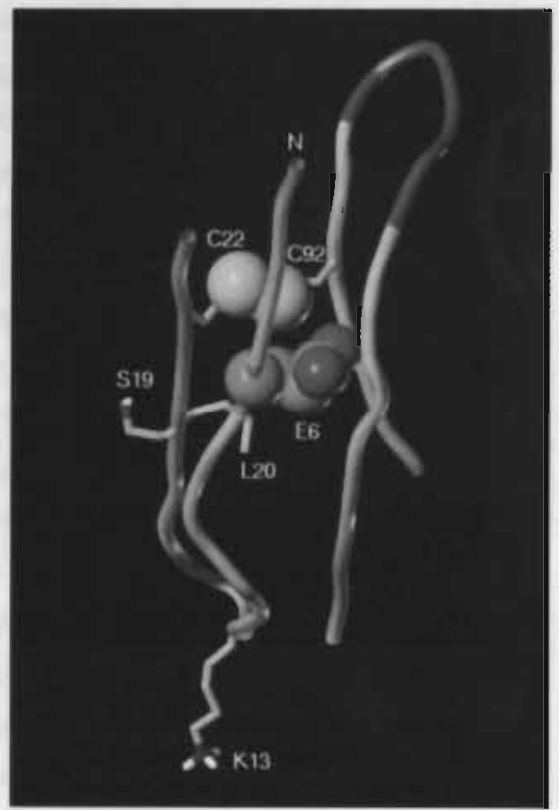

Fig. 6. (A) Presentation of the structure of antibody IBAF made with the program Sybyl (Tripos) showing the location of residue number 6 (Glu) in relation to the intradomain disulfide bond. (B) A detailed picture of the position 6 residue of $\mathrm{V}_{\mathrm{H}}$ and its neighbouring residues Thr23, Trp96, Gln105 and Gly106. (C) As B in combination with the residues targeted for mutagenesis (Lys13, Ser19 and Leu20). C $\alpha$-trace representation of the light (green) and heavy (yellow) chains of the Fv-domain. These images illustrate the buried position of residue 6 between the $\mathrm{N}$ terminus of the $\mathrm{H}$ chain and the $\beta$ strands preceding and following CDR H3 (purple). The side chain atoms of Glu 6 (carbons in orange, oxygens in red) and of the backbone NH-groups surrounding the buried sidechain (e.g. those from Cys92, Gin105, Gly106 and Glu6 itself with nitrogen in blue and hydrogen in white) are depicted as full Vanderwaals spheres. 
However, when only a single mutation was introduced, threonine on position 19, the effect was less profound than the combination of the three mutations (Table III; K19T-mutants). It thus appears possible to restore folding of the Q6E versions of these IIA antibodies by engraftment of FR1 residues that are normally found in conjunction with residue $\mathrm{E}$ at position 6.

Table III. $\mathrm{V}_{\mathrm{H}}-\mathrm{FR} 1$ mutations compensating for loss of binding in Q6E variants.

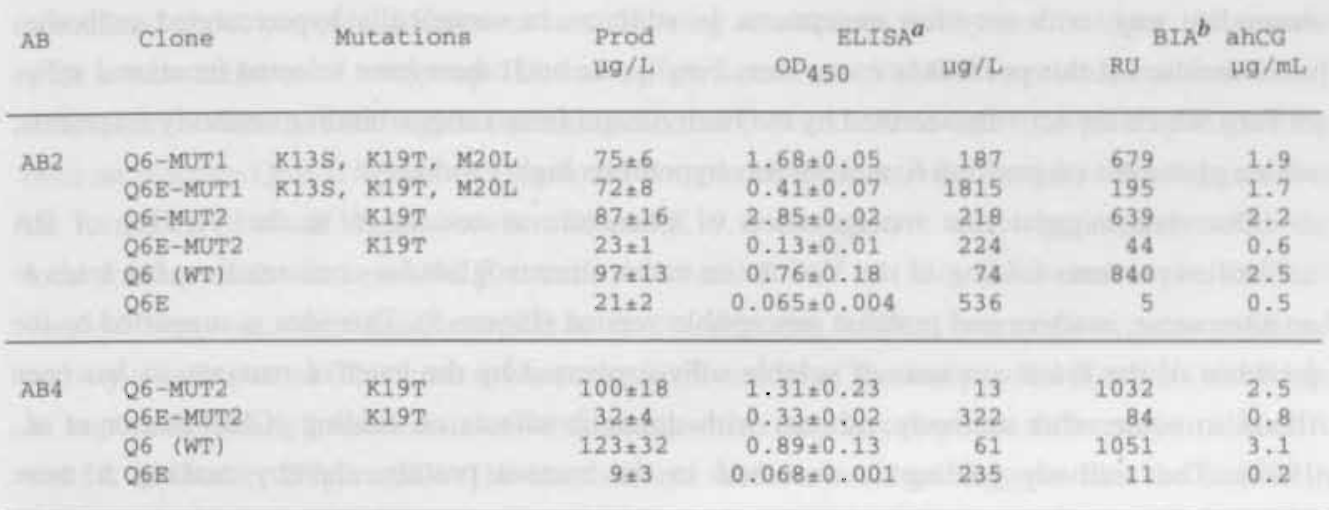

${ }^{a}$ ELISA was performed with plates coated with $10 \mu \mathrm{g} / \mathrm{ml}$ hCG. ${ }^{b}$ For BIAcore the hCGreactivities were determined on a high density coated chip ( $4369 \mathrm{RU})$ at $\mathrm{t}=400 \mathrm{~s}$.

\section{Discussion}

We demonstrate the importance of the wild type residue glutamine on position 6 of $V_{H}$ domains in three anti-hCG monoclonal antibodies recognizing different epitopes on the antigen. Introduction of glutamate, encoded by cloning site Xhol in the FRI region, abolishes binding activity, in spite of the fact that the introduced residue is also naturally occurring at this position. In addition to the antibodies described in detail here, 3 more antibodies (one additional anti-hCG and two anti-HIV-1 p24 antibodies) with a $\mathrm{V}_{\mathrm{H}}$ of class IIA were analysed; in all cases the cloned scFv versions with Q6E were not reactive. Therefore we believe that this phenomenon is a general feature for murine antibodies with a $\mathrm{V}_{\mathrm{H}}$-domain of the IIA class.

How does the glutamine residue of position 6 exert its effect on the reactivity of the antihCG antibodies? In all clones tested, we have found a complete loss of antigen binding. Since antibodies recognising completely different antigens or epitopes showed the same defects upon 
mutation, we suspect that residue 6 is not directly or indirectly involved in shaping and/or influencing the antigen binding site.

One possibility for the dramatic effect of the residue 6 point mutation would be the effect of the introduction of a charged residue within close proximity of the leader peptide, which might disturb transport over the membrane into the periplasmic space (Ayala et al., 1995). The residue 6 point mutation, however, involves an evolutionary conserved change: in murine and human antibody germline segments, either $\mathrm{Q}$ or $\mathrm{E}$ is used. From inspection of the sequences of germline $\mathrm{V}$-genes available in databases, the choice appears to have been made in a nearly familydependent way, with very few exceptions. In addition, in somatically hypermutated antibodies other residues at this position are very rare. Finally, we and others have selected functional scFvs or Fabs which are actively secreted by the bacteria and form antigen binding antibody fragments, with a glutamate on position 6 , making this hypothesis highly unlikely.

Our data suggest that incorporation of glutamate at residue 6 in the $V_{H}-F R 1$ of IIA antibodies prevents folding of the $\mathrm{V}_{\mathrm{H}}$ into its native immunoglobulin conformation. but leads to an alternative, inactive and protease susceptible version (Figure 5). This idea is supported by the presence of the lower amounts of soluble scFv expressed by the inactive mutants, as has been found in some other antibody variants with dramatic effects on folding (Glockshuber et al., 1992). The antibody folding is disturbed in the mutant protein, thereby making it more susceptible for activity of trypsin in vitro and bacterial proteases in vivo, as was seen before with antibody fragments (Glockshuber et al., 1992) and a T-cell receptor (Wulfing and Plückthun, 1994). When producing the scFv by in vitro refolding. soluble material is retrieved, however, antigen binding is never restored, indicating that the in vivo and in vitro folding process has been interfered with, and other causes of non-functionality such as differential interactions with chaperonins, effects on secretion, or aggregation rates can be excluded. Our data indicate that glutamine in antibodies with a $V_{H}$ of the class IIA is crucially involved in the folding of the antibody $\mathrm{V}$-domain, and that its exchange to glutamate interferes with folding of most if not all IIA antibodies, independent of the nature of the antigen bound by the antibody. Since this is the case for in vitro refolded protein as well, we suggest that the folding pathway (Figure 5A) has been re-routed by the Q6E mutation, thereby completely disturbing the production of correctly folded protein. The alternative pathway leads to mixes of aggregates, which are rapidly destroyed by bacterial proteases in vivo (Figure 5B), and non-aggregated, misfolded protein. This effect may be caused by destabilisation of the transition state nucleus, for example by an increase in the time in which hydrophobic areas are transiently exposed, and leads to entrapment of intermediate folding states. The effect of the mutation is, however, unique, in that it induces a complete block in the production of correctly folded protein.

The structural rolc of the position 6 residue in immunoglobulin domains was described by I.rsk and Chothia (I.esk and Chothia. 1982), although the authors confined their discussion to the residuc piesent in murin: light chain variable regions and constant domains, which is 
invariably glutamine. The side-chain of residue 6 is oriented towards the hydrophobic core of the protein, and for $\mathrm{V}$-regions at least, the side chain atoms of the residue form hydrogen bonds to the main chain atoms of the opposite $\beta$-sheet, ie. with the crucial cystein residue on position 88 , with tyrosin 86 and threonine 102. Due to the interruption of the normal $\beta$-sheet association by the bulky amide group of glutamine 6, an extra residue was inserted in the opposite strand thereby creating a $\beta$-bulge fitting around this amide group. We observed no variation in the localisation of the side chain of residue number 6 or in the formed hydrogen bonds between heavy chain V-regions with either glutamine or glutamate of murine origin, and therefore speculated about possible differences in stabilizing interactions with other residues. Alignment with a rare member of subgroup IIA having glutamate on 6 (MBRI) revealed a number of differences within the FRI region (spanning the first $\beta$ strands $A / A^{\prime}$ and $B$ ), which upon grafting onto an inactive Q6E-IIA antibody, restored antigen binding activity partially. We suggest that the formation of a salt bridge between E6 and K19 in the inactive antibody, interferes with the local folding of the anti-parallel $\beta$ strands $A / A^{\circ}$ and $B$ of FRI; this is restored in the E6, T19 version. However, more residues must be involved in this folding intermediate, since regained activity is only partial. This hints towards the involvement of the anti parallel $\beta$ strands of FRl in an important lolding intermediate or subdomain, which may be composed differently in class IIA and $\mathrm{IA}$ antibodies (Figure $5 \mathrm{C}$ ). The involvement of more residues suggests that the number 6 residue might be a constituent of a folding nucleus (Shakhnovich et al, 1996). In this folding model just a limited number of residues of a protein form most contacts in the transition state. Regarding the significance of the residues for the total folding process, it was suggested that the residues of the folding nucleus should be evolutionary conserved. Mutations affecting the stability of the transition state nucleus should have a greater effect than other mutations. In this respect the $V_{H}$ number 6 residue of the murine antibodies meets the criteria of conservation and the effect of the mutation. It was already noticed (Kirkham and Schroeder Jr., 1994) that the two solvent exposed regions within FR1 (codons 6-24) and in FR3 (codons 67-85) are highly conserved between members of a single $\mathrm{V}_{\mathrm{H}}$-family, but divergent between members of different $\mathrm{V}_{\mathrm{H}}$-families. Our data suggest that these areas might form part of a family specific folding nucleus, and that they, when completely defined, may be grafted between antibodies from different classes. However, it remains to be experimentally confirmed that grafting completely restores the "active" conformation by for instance the determination of trypsin sensitivity or by

\section{CD spectroscopy.}

Recently Plückthun and colleagues described the presence of a folding nucleus in the inner $\beta$-sheet of the $V_{L}$ of a scFv derived from McPC603 (Freund et al., 1996), but found no proof for a similar nucleus in the $\mathrm{V}_{\mathrm{H}}$. In the antibodies that we have isolated such crucial folding nucleus may exist in the $V_{L}$ also, and the $V_{L}$ may also 'chaperone' the folding of the $V_{H}$ as suggested by Pliickthun. However, since $V_{H}$-FRl residues do not display any side chains that become buried upon $V_{I}-V_{H}$ association (Freund et al., 1996), it is unlikely that the role of FRl in $V_{H}$ folding is 
to merely provide a surface for $V_{L}$ binding to assist further $V_{H}$ folding. It is more likely that by the interaction between the $V_{H}$ FRl-residues of $\beta$ strands $A / A^{\prime}$ and $B$ an early $V_{H}$ folding nucleus may be formed independent from the $\mathrm{V}_{\mathrm{L}}$ folding nucleus. and that the $\mathrm{V}_{\mathrm{L}}$ may assist in further folding of the $\mathrm{V}_{\mathrm{H}}$-domain.

For class IIB antibodies, the effect of mutation of $\mathrm{V}_{\mathrm{H}}$ residue 6 on antigen binding seems to be different. This was observed by Kipriyanov and colleagues (Kipriyanov et al., 1997). They also found a severely lowered production level of soluble Q6E mutant scFv, what was already indicated in our earlier work (De Haard and Kazemier, 1995), in fact comparable to what we observed, but its affinity was identical to the wild type fragment. If the affinities of our Q6E mutant antibody fragments derived from the IIA antibodies AB2 or AB4 would be identical to those of the wild lype fragments, we would have expected high responses in ELISA and BIAcore when used at equal concentrations as the wild type derivatives. Therefore, our data suggest that for folding, IIA antibodies require absolute conservation of residue 6 , while the studied IIB antibody is more tolerant to mutation of this residue.

Besides obvious implications on antibody expression in bacteria and possibly other expression systems, our findings will have an impact on the construction of phage antibody libraries. Mostly phage antibody repertoires are derived from immune or naive B-cells, using oligonucleotides based in the FRI and FR4 regions of the light and heavy chain V-genes (for review see Hoogenboom et al., 1992b). For amplification of murine $\mathrm{V}_{\mathrm{H}}$ genes, the frequently used degenerate primer VHIBACK (Ward et al. 1989), which primes in FR 1 covering the first 24 nucleotides, allows incorporation of either $\mathrm{Q}$ or $\mathrm{E}$ at position 6. Although preferentially the naturally occurring residue will be amplified and not the mismatched (non-WT) version, it cannot be excluded that murine IIA $V_{H}$ genes are supplemented with $E$ rather than $Q$ at position 6 , yielding non-functional antibodies. Problems caused by primers, which incorporate non wild type residues, will also arise when using 'family-specific' primers for the amplification of rearranged V-gene pools from naive or immune B-cells (Marks et al., 1991). Extensive sets of family specific primers for the amplification of murine $V$-genes have now been described by several groups (Orum et al.. 1993; Krebber et al. 1997), but combining them within a single PCR reaction might promote mismatching and thereby decrease the number of functional displayed antibody fragment within a library. Future primer design and -choice should take into account the incorporation of 'original' residues in $\mathrm{V}_{\mathrm{H}}$-FRI (as may be done for synthetic antibody libraries; Hoogenboom and Winter, 1992a), to retain essential elements of the folding nucleus, which will contribute to the creation of antibody libraries of optimal quality. 


\section{References}

Ayala.M., Balint,R.F., Fernandez de Cossio,L., Canaan Haden,J.W., Larrick,J.W., and Gavilondo,J.V. (1995) Biotechniques, 18, 832-840.

Benhar,I. and Pastan,I. (1995) J. Biol. Chem., 270, 23373-23380.

Bentley,G.A., Boulot,G., Riottot,M.M., and Poljak,R.J. (1990) Nature, 348, 254-257.

Birken,S., Krichevsky,A., O'Connor,J., Lustbader,J., and Canfield,R. (1990) in Glycoprotein Hormones: structure, synthesis and biologic function pp. 45-61, Serono Symposia.

Burton,D.R., Barbas,C.F., Persson,M.A., Koenig,S., Chanock,R.M., and Lerner.R.A. (1991) Proc. Natl. Acad. Sci. U. S. A., 88, 10134-10137.

Canfield,R.E. and Morgan,F.J. (1973) in Methods in investigative and diagnostic endocrinology (Berson,S.A. and Yalow,R.S. , Eds) pp. 727-733, North Holland Publishing Co., Amsterdam.

Carter,P., Kelley,R.F., Rodrigues,M.L., Snedecor,B., Covarrubias,M., Velligan,M.D., Wong,W.L., Rowland,A.M., Kotts,C.E., Carver,M.E., Yang,M., Bourell,J.H., Shepard.H.M., and Henner,D. (1992) Biotc'chology (N Y), 10, 163-167.

Chirgwin,J.M., Przybyla,A.E., MacDonald,R.J., and Rutter,W.J. (1979) Biuchcmistry, 18, 5294.

Chothia,C., Lesk,A.M., Gherardi,E., Tomlinson,I.M., Walter,G., Marks,J.D., Llewelyn, M.B., and Winter,G. (1992) J. Mol. Biol., 227, 799-817.

Clackson,T., Hoogenboom,H.R., Griffiths,A.D., and Winter.G. (1991) Nature, 352, 624-628.

De Haard,H., and Kazemier,B. (1995) Hum. Antibodies Hybridomas, 6, 36.

Dower,W.J., Miller,J.F., and Ragsdale,C.W. (1988) Nucleic Acids Res., 16, 6127-6145.

Foote.J., and Winter,G. (1992) J. Mol. Biol., 224, 487-499.

Freund,C., Honegger,A., Hunziker,P., Holak,T.A.. and Plückthun,A. (1996) Biochemistry, 35, 8457-8464.

Glockshuber,R., Schmidt,T., and Plückthun,A. (1992) Biochemistry. 31, 1270-1279.

Hawkins, R.E.. Russell,S.J., Baier,M., and Winter,G. (1993) J. Mol. Biol., 234, 958464.

Herron.J.N., He.X.M., Mason,M.L., Voss,E.W.J., and Edmundson.A.B. (1989) Proncins, 5, 271-280.

Hoogenboom.H.R., Griffiths.A.D., Johnson,K.S., Chiswell,D.J.. Hudson.P., and Winter,G. (1991) Nucleic Acids Res., 19,4133-4137.

Hoogenboom,H.R. and Winter.G. (1992a) J. Mol. Biol., 227, 381-388.

Hoogenboom,H.R., Marks,J.D., Griffith,A.D., and Winter,G. (1992b) Immunol. Rev., 130, $41-68$.

Hopp,T.P. Prickett,K.S., Price.V.L., Libby,R.T., March,C.J., Cerretti,D.P., Urdall.D.L., and Conlon,P.J. (1988) Biotechnology (N Y), 6, 1204-1210. 
Horton,R.M., Hunt,H.D., Ho,S.N., Pullen,J.K., and Pease,L.R. (1989) Gene, 77, 61-68.

Jayaraman,K., Shah,J., and Fyles,J. (1989) Nucleic Acids Res., 17, 4403.

Jonsson,U., Fagerstam,L., Ivarsson,B., Johnsson,B., Karlsson,R., Lundh,K., Lofas,S.,

Persson,B., Roos,H., Ronnberg,I., et al. (1991) Biotechniques, 11, 620-627.

Kabat,E.A., Wu,T.T., Reid-Miller,M., Perry,H.M., and Gottesman,K.S. (1991) Sequences of proteins of immunological interest, $5^{\text {th }} \mathrm{Ed}$., US Department of Health and Human

Services, US Government Printing Office, Bethesda, MA.

Kipriyanov,S.M., Moldenhauer,G., Martin,A.C., Kupriyanova,O.A., and Little,M.

Protein Eng., 10, 445-453.

Kirkham.P.M. and Schroeder Jr.,H.W. (1994) Semin. Immumol., 6, 347-360.

Knappik,A. and Plückthun,A. (1995) Protein Eng., 8, $81-89$.

Krebber,A., Bornhauser,S., Burmester,J., Honegger,A., Willuda.J.. Bosshard,H.R., and

Plückthun, A (1997) J. Immunol. Methods, 201, 35-55.

L.eahy,D.J., Hynes,T.R., McConnell,H.M., and Fox.R.O. (1988) J. Mol. Biol., 203, 829-830.

Lesk,A.M, and Chothia,C. (1982) J. Mol. Biol., 160, 325-342.

Marchuk,D.. Drumm,M., Saulino,A., and Collins.F.S. (1990) Nucleic Acids Res., 19, 1154.

Marks.J.D., Hoogenboom.H.R.. Bonnert.T.P.. McCafterty.J.. Griffiths.A.D., and Winter.G. (1991) J. Mol. Biol., 222, 581-597.

Minsky,A., Summers,R.G., and Knowles,J.R. (1986) Proc. Natl. Acad. Sci. U. S. A., 83, 4180-4184.

Orlandi.R., Gussow.D.H.. Jones.P.T., and Winter,G. (1989) Proc. Natl. Acad. Sci. U. S. A., 86, 3833.3837 .

Orum,H., Andersen,P.S., Oster,A., Johansen,L.K., Riise,E., Bjomvad,M., Svendsen,I., and Engberg,J. (1993) Nucleic Acids Res., 21, 4491-4498.

Padlan,E.A., Silverton,E.W.. Sheriff,S., Cohen,G.H., Smith Gill.S.J., and Davies,D.R. (1989) Proc. Natl. Acad. Sci. U. S. A., 86, 5938-5942.

Riechmann.L., Clark,M., Waldmann,H., and Winter,G. (1988) Nature, 332, 323-327.

Shakhnovich,E., Abkevich.V., and Ptitsyn,O. (1996) Nature. 379, 96-98.

Sheriff,S., Silverton,E.W., Padlan,E.A., Cohen,E.A., Smith-Cill.S.J., Fin/el.B.C., and Davies,D.R. (1987) Proc. Natl. Acad. Sci. U. S. A., 84, 80758079.

Skerra.A. and Plückthun,A. (1991) Protein Eng., 4, $971-979$.

Tomlinson,I.M., Walter,G., Marks,J.D., Llewelyn,M.B., and Winter.G. (1992) J. Mol. Biol., 227, 776-798.

Tramontano,A., Chothia,C., and I.ekk.A.M. (1990) J. M/ol. Biol., 215, 175-182.

Verhoeyen,M., Milstein,C., and Winter.G. (I989) Sicience, 239, 1534-1536.

Ward.E.S.. Gussow.D., Grilliths,A.D.. Jones.P.T., and Winter.G. (1989) Nature, 341, 544546.

Wulfing,C. and Plückthun,A. (1994) J. Mol. Biol, 242, 655-669. 
Zacher,A.N., Stock,C.A., Golden,J.W., and Smith,G.P. (1980) Gene, 9, 127-140.

\section{Acknowledgements}

We are grateful to Peter van Weezenbeek (Organon) for giving us the phagemid vector pVI, Bert van der Weijer for purified hCG and for practical instructions concerning ELISAs and Richard Peters, Ine van Kuijk. Yvette de Hingh and Liesbeth van Kerkhoff for cloning the variable regions and protein analysis. Finally, Dick Schaap for critical reading of the manuscript. This work was supported by PBTS grant BIO 91028. 


\section{Chapter 2}

\section{A FR1 MUTATION IN VL AFFECTING ANTIGEN BINDING}

In press

Residue 4 in the Vernier Zone of mouse antibodies with a subgroup II kappa light chain is a critical determinant for antigen recognition

Hans J.W. de Haard, Bert Kazemier, Arie van der Bent, Peter Oudshoorn, Piet Boender, Jan-Willem Arends, and Bob van Gemen

Immunotechnology 


\section{Abstract}

Background: During the conversion of murine monoclonal antibodies directed against the human chorionic gonadotropin (hCG) into bacterially expressed single chain fragments (scFv), we found a major reduction of binding activity upon introduction of a primer encoded mutation. Objectives: In this study we tried to determine which mutation was responsible, and on what manner this mutation affected antigen binding (structural effect versus direct involvement of the residue in binding). Results: No binding could be detected, when the wild type residue methionine at position 4 within the Framework region 1 of the VK light chain was substituted by serine in two antibodies with a subgroup II kappa light chain. However, a similar replacement within an anti-hCG antibody with a subgroup IV kappa light chain, and thereby having leucine as wild type residue, did not affect the binding characteristics. The mutant scFv's derived from both AB-s sensitive for substitution by serine never reacted with antigen in ELISA. By using Surface Plasmon Resonance, a residual binding was observed only under conditions, when a high density antigen coating was applied; however, an increased dissociation, rclative to that of the wild type scFv, and the absence of reactivity in ELISA suggest a drastically altered affinity. Conclusion: A structural explanation for the changed binding characteristics can be the influence of the position 4 residue, as being a constituent of the Vernier Zone, on the position of the CDR I loop of $\mathrm{VK}$, which might harbour residues that directly bind to antigen, or indirectly positions other variable loops of the binding pocket. An increased sensitivity for trypsin digestion supported the hypothesis of a locil conformational change in the serine mutant of the subgroup II kappa containing antibody.

\section{Introduction}

The binding part of an antibody consists of the amino terminal domain of the heavy (VH) and light chain (VL), which are closely associated by non-covalent interactions. The relatively conserved framework residues (FR) of the variable domains are involved in the firmation of a nine-stranded antiparallel $\beta$ barrel conformation. The three hypervariable Inops of auch domain. forming the complementarity determining regions (CDRs), connect the $\beta$-strands at the aminoterminal end of the barrel and harbour the antigen contacting and binding residues.

Besides providing a scaffold for mounting the CDR-loops. FR residues occisionally make direct contacts with antigen (Sheriff et al., 1987; Padlan et al., 1989; Bentley et al., Iy9U), or on the other hand, they can affect binding indirectly by positioning of the CDR (Chothia et al., 1989: Tramontano et al. 1990). Chothia and Lesk (1987) described the influence of particular framework residues on the main chain conformation of the hypervariable loops. The defined set of canonical structures of CDR loops seems to be determined by the size and the presence of 
well defined residues at particular sites within the loop but also in the framework regions (Chothia et al., 1989). During the humanisation of antibodies, where murine CDR loops are grafted on a human framework, it is often necessary to include a number of murine framework residue(s) for retaining the parental affinity (Riechmann et al., 1988). By making key contacts with CDRs these framework residues help to maintain the conformation of the loops. The critical residues, the so called Vernier residues, are often located on the borders of the CDRs and were analysed in more detail (Foote and Winter, 1992).

For the production of antibodies or fragments thereof in mammalian cells or in bacteria, the variable region (V-) genes are amplified by the polymerase chain reaction (PCR) with "universal" primers suitable for the isolation of a range of heavy and light chain sequences. The design of primers in the FR4 region is relatively easy, since this area is encoded by a limited number homologous joining (J-) segments. However, FRI primers are family specific and have to be used stparately (Marks et al., 1991) or were synthesized as degenerate oligonucleotides (Orlandi et al., 1989; Sastry et al., 1989). Restriction sites, rarely occurring in natural antibody variable sequences, were often introduced to facilitate cloning (Sastry et al., 1989; Orlandi et al., 1989; McCaflerty it al., 1990). As a consequence of degeneracy and of the introduced sites, the primer encoded regions will contain non wild type residues, and especially for the amino terminal regions this might be critical for the binding characteristics (Padlan, 1991).

We already observed the drastic effect of an evolutionary conserved substitution of the position 6 residue in $\mathrm{VH}$ during the conversion of two different mouse antibodies recognizing the human chorionic gonadotropin (hCG) into bacterially expressed scFv antibodies (de Haard et al., 1998). When the wild type residue glutamine, present in antibodies with a subgroup IIA heavy chain (Kabat et al., 1991), was mutated into glutamic acid, mainly found in class IA heavy chains, antigen binding was completely lost as the result of misfolding.

Here we describe the effect of a mutation within the amino terminal region of the kappa light chain caused by the introduced restriction site Sall. When the position 4 residue methionine of two anti-hCG antibodies with a subgroup II kappa light chain was replaced by serine, antigen binding was abolished completely as was measured in ELISA. In contrast, one other hCG antibody with a class IV kappa chain tolerated the introduced serine. Analysis of the structure of the mutant antibodies (AB-s) by trypsin digestion suggested that a local distortion of the conformation might be responsible for a drop in affinity.

\section{Materials and methods}

\subsection{Cloning and screening}

The murine monoclonal antibodies (lgGl, kappa) used for the conversion into bacterially produced scFv's are coded $A B 2, A B 3$ and $A B 4$ and recognize the $\beta$-core fragment, the $\beta$ - 
Carboxy Terminal Peptide (CTP), and $\beta$-core fragment of the gonadotropin hCG (nomenclature see Birken et al., 1990). The amplified variable regions were cloned in the phagemid vector pVI, which is identical to pHENI except with a FLAG- (Hopp et al., 1988) instead of the Myc-tag. More experimental details including the sequences of the used primers can be found elsewhere (de Haard et al., 1998).

Briefly, the antibody V-regions were initially cloned with degenerated primers containing restriction sites introduced within the variable regions (a Xhol- or Pst-site in the VH FR1, NcoI in VH FR4, SalI in the VK FRI and a BcIl-site within VK FR4). After electroporation (Dower et $a l ., 1988)$ in the $E$. coli non suppressor strain TOP F', individual clones were screened with PCR using the M13reverse and the genellI-primer. Transformants containing the expected inserts were grown in $50 \mathrm{ml}$ medium and induced (Marks et al., 1991). The scFv containing periplasmic fractions, prepared by the shock procedure with borate buffered saline (Skerra and Plückthun, 1991), were analysed in ELISA and surface plasmon resonance for binding to hCG (for experimental details see (de Haard et al., 1998). Kinetic analysis was performed with surface plasmon resonance using the BIAcore ${ }^{\mathrm{TM}}$; CM-chips (Biacore $\mathrm{AB}$, Lund, Sweden) were applied for covalent coupling of hCG. Experiments were performed with a high density coat of hCG (4369 response units (RU); molecular weight of $45 \mathrm{kD}$ ).

The wild type constructs, encoded WT, were made by amplification with a VH FRI primer extended into the pelB leader sequence and containing a Sfil-site, while the VK FR4 primer with a Notl-site was extended to the FLAG-sequence as described (Clackson et al., 1991). The heavy and light chain V-genes were assembled with the linker fragment in a splicing by overlap extension polymerase chain reaction (SOE-PCR) (Horton et al. 1989) and cloned as Sfil/Not Lfragments. TOP F' transformants were screened by PCR as above and used for the expression of soluble scFv.

A similar cloning strategy was applied for the generation of phage libraries. Again. amplification was performed with extended primers containing the SfiI- and NotI-sites, which were degenerated to some extent. After transformation of the suppressor strain JM101, phagemid particles were rescued as described (Marks et al., 1991). After two panning rounds on purified hCG, coated at $3 \mu \mathrm{g} / \mathrm{ml}$ on polystyrene plates. clones (encoded $\mathrm{PH}$ ) producing functional scFv were detected by ELISA using the culture supernatants. Phage was prepared from functional clones and was used for transfection of TOP F cells. The resulting transformants were grown and induced as described before.

Mutants AB4-V2I and AB4-M4S were constructed by application of SOE PCR with primers encoding the mutation and having an introduced diagnostic (silent) restriction site; the number of cycles was limited to 20. The scFv encoding inserts of the clones characterised on binding were completely sequenced. 


\subsection{Trypsin digestion}

The stability of the scFv produced by the serine mutant of AB4 (AB4-M4S) could be directly compared with the wild type scFv of $\mathrm{AB} 2$ by combining both periplasmic fractions, containing approximately $50 \mathrm{ng}$ antibody fragment, into a single digestion reaction containing $100 \mathrm{mM}$ Tris- $\mathrm{HCl}$ pH8.0 and $10 \mathrm{mM} \mathrm{MgCl}_{2}$ and 200 or $40 \mu \mathrm{g} / \mathrm{ml}$ (ICN Biomedicals Inc, Costa Mesa, CA, USA). Alternatively, a mixture was made of the extracts from clones AB4-M4S and AB2-Q6E. After 2 hours incubation at $16^{\circ} \mathrm{C}$ the digestion was stopped by adding Laemmli buller. The scFv fragments derived from the two different AB-s were separated on a $12 \%$ polyacrylamide gel. After blotting the products were detected with anti-FLAG M2 antibody (Kodak IBI. New Haven, USA).

\section{Results}

\section{I Identification of a VK-FRI mutation affecting antigen binding}

A panel of murine anti-hCG mABs, of which the light chain sequences are shown in Fig. I, was converted into bacterially expressed scFv's. Initially the V-regions were amplified and cloned with primers containing introduced restriction sites. For the antibody coded AB2, which is reactive against the $\beta$ core fragment of hCG, problems were encountered with respect to the production of functional $\mathrm{scFv}$. This clone was analysed in more detail. Individual primer encoded FR-regions were exchanged to wild type, and it appeared that when the mutant VK FRI was replaced, the produced antibody fragments were reactive in ELISA against hCG (Table I).

\section{Light chain}
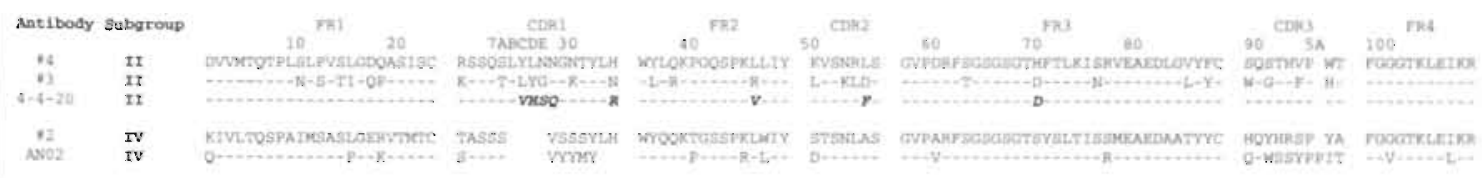

Figure 1. Deduced amino acid sequences of the variable light chain regions of anti-hCG monoclonal antibodies $A B 2$. AB3 and AB4. Numbering of the residues and light chain classification was performed according to Kabat $\mathrm{ct}$ al. (1991). In addition the VK sequences have been aligned of the anti-2,2,6,6-tetramethyl-I-piperidinyloxy-dinitrophenyl antibody ANO2 (panel above) and the anti-2-methyl-2,4-pentanediol antibody 4-4-20 (panel below).

This region was examined more closely. Only two mutations could be responsible: at position 2, where the wild type valine was replaced by isoleucine, and at position 4 , where methionine was substituted by serine. The activity of two new variants, with the complete wild type sequence except on position 2 (clone V2I) or 4 (clone M4S), showed that serine on position 4 is crucial for antigen binding (Table I). 
Another antibody coded AB3, directed against the carboxy terminal part of the $\beta$ subunit of hCG, and which shares the Vk-family (class II) with $\mathrm{AB} 4$, was analysed in a similar way. Here also the mutation M4S abolished antigen reactivity as measured in ELISA (results discussed below). It is interesting to see that $A B 2$, reactive against hCG $\beta$ core fragment and with a $V_{K}$ region of a different family (class IV), tolerates the substitution of its wild type residue leucine by serine (see results below).

Table I. Determination of the additional primer encoded FR-region in AB4 responsible for loss of antigen binding.

\begin{tabular}{|c|c|c|c|c|c|c|c|c|}
\hline \multirow[t]{2}{*}{ Clone } & \multicolumn{4}{|c|}{$\mathrm{VH}^{a}$} & \multicolumn{3}{|r|}{$\mathrm{vk}^{\mathrm{a}}$} & \multirow[t]{2}{*}{ ELISA $^{b}$} \\
\hline & & FR1 & & FR4 & & FR1 & FR4 & \\
\hline HF 4-A & & $W T$ & & MUT & & MUT & MUT & - \\
\hline $\mathrm{HF} 4-\mathrm{C}$ & & WT: & & MUT & & MUT & WT & - \\
\hline HF 4-D & & WT: & & WT & & MUT & WT & - \\
\hline HF $4-B$ & & WT & & MUT & & WT & MUT & + \\
\hline HF $4-E$ & & WT & & MUT & & WT & WT & + \\
\hline V2I & & WT: & & MUT & $\operatorname{Val}(2$ & (2) $\rightarrow$ Ile & MUT & + \\
\hline M4S & & WT: & & MUT & $\operatorname{Met}(4$ & (4) $\rightarrow$ Ser & MUT & - \\
\hline \multicolumn{9}{|c|}{ Encoded amino acid sequences: } \\
\hline & $\mathrm{VH}$ & FR1 WT & : & QVQLQQSG; & $\begin{array}{l}\text { VH FR4 } \\
\text { VH FR4 }\end{array}$ & $\begin{array}{l}\text { WT: : WC } \\
\text { MUT: WC }\end{array}$ & $\begin{array}{l}\text { WGQGTLVTVSA; } \\
\text { WGQGTMVTVSS; }\end{array}$ & \\
\hline & $V_{\kappa}$ & FRI WT & : & DVVMTQTP: & VK FR4 & WT : GT & GTKLEIK & \\
\hline & $V \kappa F$ & FR1 MUT & : & EIVSTQSP; & VK FR4 : & MUT: $:$ G? & GTKLVIK & \\
\hline
\end{tabular}

In conclusion, we observed that the substitution of methionine on position 4 of $\mathrm{VK}$ by serine in $A B 3$ and $A B 4$, both having a subgroup II kappa light chain. was associated with negative ELISA results. In contrast the class IV kappa light chain containing AB2 with leucine as wild type residue tolerates the serine residue introduced by a Sall site. We examined the production levels of all mutants and quantified the reactivity in ELISA and with surface plasmon resonance. 


\subsection{Analysis of reactivity}

The scFv concentration in the periplasmic fractions was determined on Western blot with the anti-FLAG antibody. The measured sample signal was compared with the signals of a range of purified scFv standards present on the same blot.

All periplasmic fractions were tested in ELISA (Table II) and surface plasmon resonance (Fig. 2) for binding with hCG. The most striking results were obtained with the mutant proteins encoded by the AB4 constructs containing serine on position 4 (clones HF4-A and M4S).

Table II. Antigen binding characteristics and FR1 sequences of the recombinant scFv's.

\begin{tabular}{|c|c|c|c|c|c|c|c|}
\hline \multirow[t]{2}{*}{$A B$} & \multirow[t]{2}{*}{ Clone } & \multirow{2}{*}{$\begin{array}{l}\text { sequence } \\
\text { FR1-VK }\end{array}$} & \multirow{2}{*}{$\begin{array}{l}\text { Proda } \\
\mu g / L\end{array}$} & \multicolumn{2}{|c|}{ ELISA $b$} & \multicolumn{2}{|c|}{ BIA $\alpha$ ahG $^{C}$} \\
\hline & & & & $\mathrm{OD}_{450}$ & $\mu g / L$ & $\mathrm{RU}$ & $\mu \mathrm{g} / \mathrm{ml}$ \\
\hline \multirow{4}{*}{ AB2 } & & 12345678 & & & & & \\
\hline & WT: & KIVLTQSP & $355 \pm 42$ & $0.37 \pm 0.04$ & 178 & 320 & 1.8 \\
\hline & HF2 & E--S- - & $256 \pm 59$ & $1.45 \pm 0.10$ & 128 & 870 & 5.1 \\
\hline & & 12345678 & & & & & \\
\hline \multirow[t]{7}{*}{$\mathrm{AB} 4}$. & WT & DVVMTQTP & $462 \pm 14$ & $2.22 \pm 0.06$ & 116 & 1438 & 2.3 \\
\hline & $\mathrm{PH} 1$ & $E--L--S-$ & $153=30$ & $2.45 \times 0.16$ & 38 & 683 & 0.8 \\
\hline & PH2 & $\mathrm{K}--\mathrm{L}--\mathrm{S}-$ & $140 \pm 35$ & $1.71 \neq 0.25$ & 35 & 615 & 0.7 \\
\hline & $\mathrm{V} 2 \mathrm{I}$ & $-I \ldots$ & $411 \div 53$ & $2.33=0.09$ & 103 & 1554 & 2.2 \\
\hline & $\mathrm{HF} 4-\mathrm{A}$ & EI-S--S- & $52.0 \pm 6.9$ & $0.072 \pm 0.008$ & 260 & 270 & 1.0 \\
\hline & M4S & $\ldots-S-\cdots$ & $15.0 \pm 4.0$ & $0.073 \pm 0.008$ & 75 & 141 & 0.3 \\
\hline & & 12345678 & & & & & \\
\hline \multirow[t]{3}{*}{$A B 3$} & WT & DVVMTQTP & & & & & \\
\hline & PH1 & $E--L-C-S-$ & $30^{d}$ & $2.75=0.00$ & 150 & 382 & 0.3 \\
\hline & $\mathrm{HF} 3-\mathrm{A}$ & BI-S- - S- & $4^{d}$ & $0.065=0.006$ & 20 & 16 & 0.04 \\
\hline
\end{tabular}

\footnotetext{
$a$ The amount of scFv was determined on western blot using a standand curve of purified scFv, ${ }^{b}$ For ELISA plates were coated with $4 \mu \mathrm{g} / \mathrm{ml}$ hCG: background signals were in the $0.05-0.08$ sange, the used concentrations of scFv in ELISA are indicated. $C$ Surface plasmon resonance analysis was performed with a high density coat of hCG (4369 RU) (see Fig. 1). The hCG-reactivity was determined at timepoint $t=400 \mathrm{~s}$. ${ }^{d}$ Single observation.
}

When tested in ELISA no signals above those of the negative controls were found, however, with surface plasmon resonance we identified distinct signals. For the VK FRI mutants residual binding was clearly detectable (clones AB4-HF4-A and AB4-M4S in Table II). Indeed, the sen- 
(A)

Increased off-rate Ser4-mutant

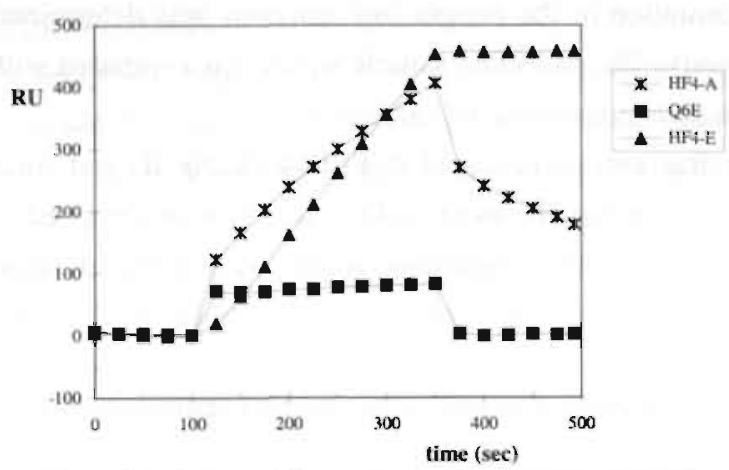

(B)

Position 4 mutants of $\mathrm{AB} 3$

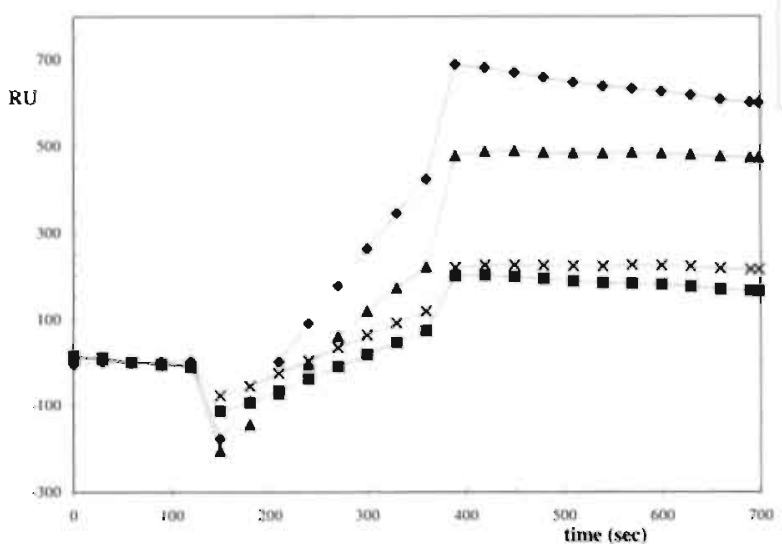

(C)

Compensation mutant AB4-M4S

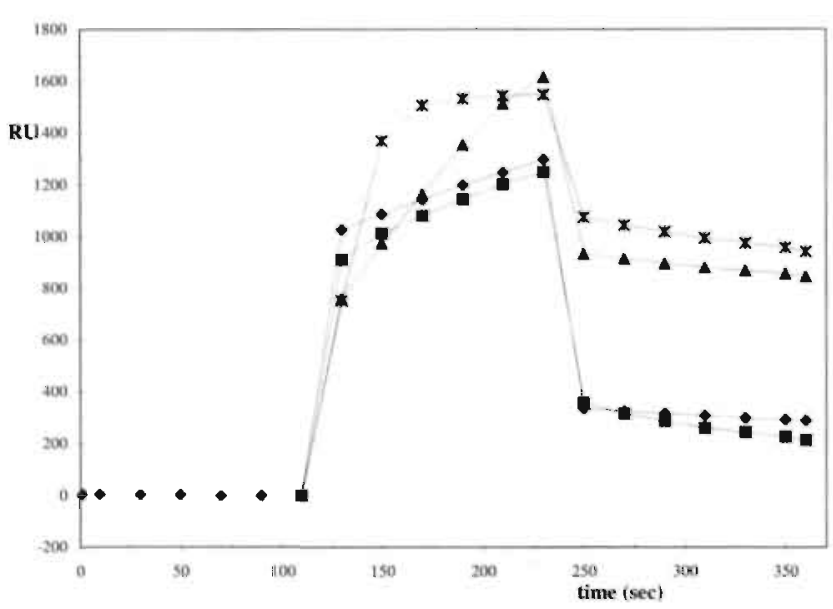


Figure 2. (Previous page) Analysis of the scFv's on hCG binding with surface plasmon resonance (BIAcore).

(A) Binding profiles of the AB4 derivatives with serine on position 4 (M4S: clone AB4-HF4-A) revealing a fast dissociation rate. The binding profiles of two other $A B 4$ derivatives (M4: functional scFv from clone $A B 4-$ HF4-E; Q6E: non-functional scFv from clone $A B 4-Q 6 E$ ) are also shown. At $t=100$ sec the association phase started by injection of periplasmic fraction. At $\mathrm{t}=340 \mathrm{~s}$ the surface was washed with Hepes-buffered saline (HBS) buffer.

(B) Overlay plot of position 4 mutants of AB3. Injection was started at $t=140 \mathrm{sec}$, and washing at $t=400$ sec. The serine containing scFv (M4S: clone AB3-HF3-A) also gave faster dissociation rates than the control (M4L: clone AB3-PH1 with leucine).

(C) Improved off-rate of $\mathrm{AB} 4-\mathrm{M} 4 \mathrm{~S}$ mutant as the result of substitution of residue number 24 arginine by threonine (clone M4Smut). Overlay plot of four AB4 derivatives injected at $t=110$ sec, washing started at $\mathrm{t}=$ $240 \mathrm{sec}$.

sorgrams gave association profiles indicating that scFv binds to $\mathrm{hCG}$, but these also revealed a faster dissociation than seen with the WT clones (compare the sensorgrams of clone AB4-HF4-E having methionine as residue number 4 with clone $\mathrm{AB} 4-\mathrm{HF} 4-\mathrm{A}$ with serine in Fig. $2 \mathrm{~A}$ ). It has to be emphasised, that the associations were only found when high concentrations scFv $(0.3 \mu \mathrm{g} / \mathrm{ml}$ or more) were injected on high density surfaces, which is an indication for a low affinity interaction. Upon dilution the signal of the serine containing clone was reduced more rapidly: from 700 to $200 \mathrm{RU}$ by a twofold dilution for AB3-HF3-A with serine, contrasting the decrease from 500 to $200 \mathrm{RU}$ by a fivefold dilution of AB3-PH1. In contrast to surface plasmon resonance, the bound scFv molecules are not detected in ELISA (Table II), probably as the result of extended washes. In conclusion, the M4S mutation reduces the affinity of binding in $A B 4$. The substitution by leucine did not affect binding, as can be seen with the mutants AB4-PHI and $-\mathrm{PH} 2$.

AB3, which like $A B 4$ has a class II $V_{K}$ light chain, also gave no positive signals in ELISA when methionine was replaced by serine (clone HF3-A in Table II). Again, leucine did not influence binding (clone $\mathrm{AB} 3-\mathrm{PH}$ ). In surface plasmon resonance binding profiles were obtained on high density surfaces only, which revealed again more enhanced dissociations than observed with the functional derivatives (Fig. 2B).

The sensitivity of the hCG ELISA should be sufficient for the detection of the scFv's expressed by the $V_{\kappa}$ position 4 mutants. For instance, $A B$ fragments produced by the serine containing clone AB4 HF-4A gave no specific signals when tested at a concentration of 260 $\mathrm{ng} / \mathrm{ml}$, while the wild type scFv gave an OD of 2.22 even at $116 \mathrm{ng} / \mathrm{ml}$.

By substitution of methionine with serine in the light chain of $A B 3$ and $A B 4$, scFv's were produced that did not react in ELISA, but in BIAcore we obtained sensorgrams, which might suggest a low affinity binding. We previously identified a mutation on position 6 of $\mathrm{VH}$, that completely abolished antigen recognition. When the wild type residue glutamine of antibodies with a class IIA VH was substituted by glutamic acid, antigen binding could not be demonstrated. The position 6 VH mutants never showed such residual antigen binding with surface plasmon resonance (clone AB4-Q6E in fig. 2A) as the result of incorrect folding, what 
was demonstrated by an increased sensitivity for trypsin digestion. It is interesting to examine the conformation of the antibody fragments produced by the position $4 \mathrm{VK}$ mutants in respect to their residual binding activity.

\subsection{Analysis of conformation by trypsin digestion}

The stability of the scFv fragments expressed by the position $4 \mathrm{VK}$ mutants was analysed by trypsin digestion as was done in other studies (Minsky et al., 1986); Glockshuber et al., 1992). By combining the periplasmic fractions in a single digestion mixture we could directly compare

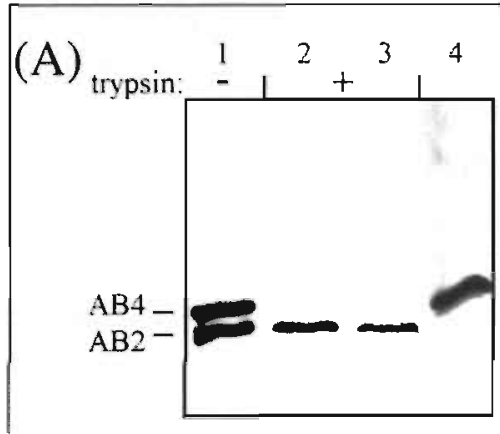

AB2-WT

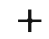

AB4-Q6E

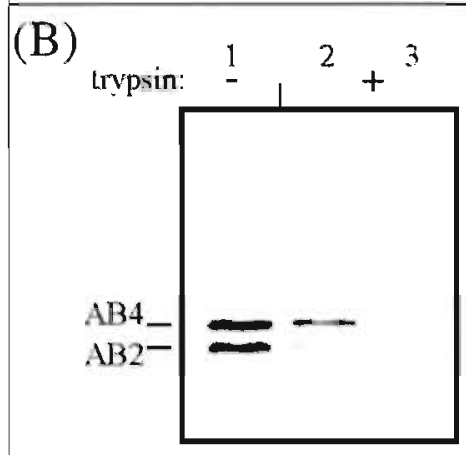

AB4-M4S

$+$

AB2-Q6E

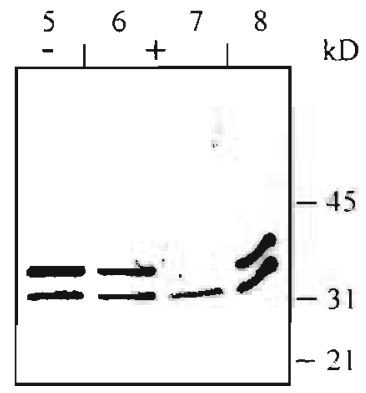

AB2-WT

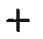

AB4-M4S

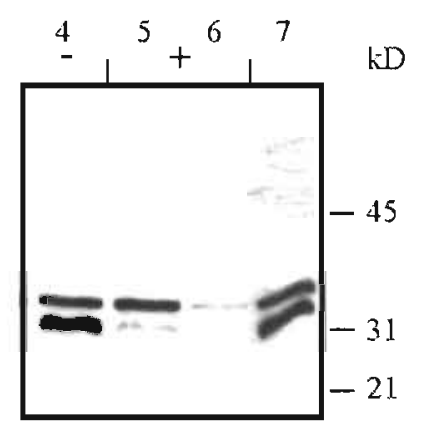

AB4-WT

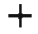

AB2-Q6E
Figure 3. Trypsin accessibility of seFv fragments of position $4 \mathrm{VK}$ and position $6 \mathrm{VH}$ mutants derived from $A B 2$ and AB4. Trypsin addition is indicated with + and -; the samples of lanes 2 and 6 in fig. A and of lanes 2 and 5 in fig. B were incubated with $0.2 \mathrm{mg} / \mathrm{ml}$ trypsin. those from lanes 3 and 7 in fig. $A$ and in lames 3 and 6 in fig. B with $40 \mathrm{mg} / \mathrm{ml}$. Lane 4 (AB4. Q6E). lane 7 (AB4-M4S and $A B 2-W T)$ in fig. $A$, and lane 7 (AB2-Q6E and AB4-WT) are controls (no digestion).

the stability of wild type and mutant scFv's. The scFv fragments from $A B 2$ and $A B 4$ were separated by electrophoresis and detected on blot with anti-FLAG. The AB fragment of clone 
AB4-M4S showed to be more trypsin resistant than those of clones AB4.Q6E (Fig. 3A) on AB2Q6E (Fig. 3B), but less than the scFv's of AB2-WT (Fig. 3A) or AB4-WT (Fig. 3B). The scFv's of the Q6E mutants degraded more rapidly because these molecules do not have the protective immunoglobulin fold. The M4S mutant has an intermediate position in terms of trypsin resistance. Probably the mutant molecule adapts the immunoglobulin fold, but the mutation has a more local effect restricted to the FRI region of $\mathrm{Vk}$.

\subsection{Compensating mutations for the S(4) mutant in VK of $A B 4$}

The fact that only antigen binding of $\mathrm{AB} 3$ and $\mathrm{AB} 4$ suffered from introduction of serine at position 4, and not $\mathrm{AB} 2$, already indicates that this residue may generically influence the binding affinity of light chain variable domains of the kappa IU family. We further studied the effect that additional mutations in the antibody scaffold might have on restoring the expression and binding of the AB4-M4S variant.

Examination of the structure of antibody AN02 directed against 2.2.6.6-tetramethyl-1piperidinyloxy-dinitrophenyl (PDB-code 1BAF (Leahy et al., 1988), aligned sequence in Fig. 1) with leucine at position 4 (Fig. 4B) showed that its neighbouring residues are the non polar alanine25 in CDR1 and threonine97 in CDR3. In contrast, the structure of a murine 4-4-20 antibody directed against 2-methyl-2,4-pentanediol (PDB-code 4FAB (Herron et al, 1989), xyuence in Fig. 1) with methionine on position 4 of VK revealed serine 25 and again threonine97 as neighbouring residues, as in antibodies $\mathrm{AB} 4$ and $\mathrm{AB} 3$, which are both sensitive for the M4S mutation. In $\mathrm{AB} 2$ the residue number 97 is the apolar alanine. Therefore we speculate that the presence of these two alanines (and thereby preventing interactions between the polar residues of positions 25 and 97 with residue 4) may make the antibody resistant to the M4S change, and we mutated serine 25 and threonine 97 of AB4 into alanine, in the context of the wild type methionine or the mutant serine on position 4. The versions with both M4S and T97A mutations, in combination with $\mathrm{S} 25 \mathrm{~A}$ or alone. were completely inactive as observed with surface plasmon resonance. This indicates that the threonine to alanine mutation in CDR3 predictably reduces binding. The version with $\mathrm{M} 4 \mathrm{~S}$ and $\mathrm{S} 25 \mathrm{~A}$ mutations gave an identical association and dissociation curve in surface plasmon resonance analysis as the M4S mutant. On the contrary, the wild type constructs (methionine at 4 ) with the alanines (single and double mutants) gave profiles similar to AB4-WT (somewhat enhanced dissociation). Thus these mutations do not affect antigen binding (despite their position in the CDR regions), but can not restore activity of the $M 4 S$ variant.

A second hypothesis was based on differences in residue 24 of $\mathrm{V} \kappa$, which is positioned nearby residue 4. In antibody 4-4-20 residue 24 is a positively charged residue (as in $A B 3$ and $\mathrm{AB} 4$ ), while antibody $\mathrm{AN} 02$ and $\mathrm{AB} 2$ have polar residues (respectively serine and threonine) on this position. We decided to substitute arginine 24 by threonine in AB4. The $\$ 4$ mutant having threonine gave a dissociation in surface plasmon resonance analysis, which is slightly better 
(A)

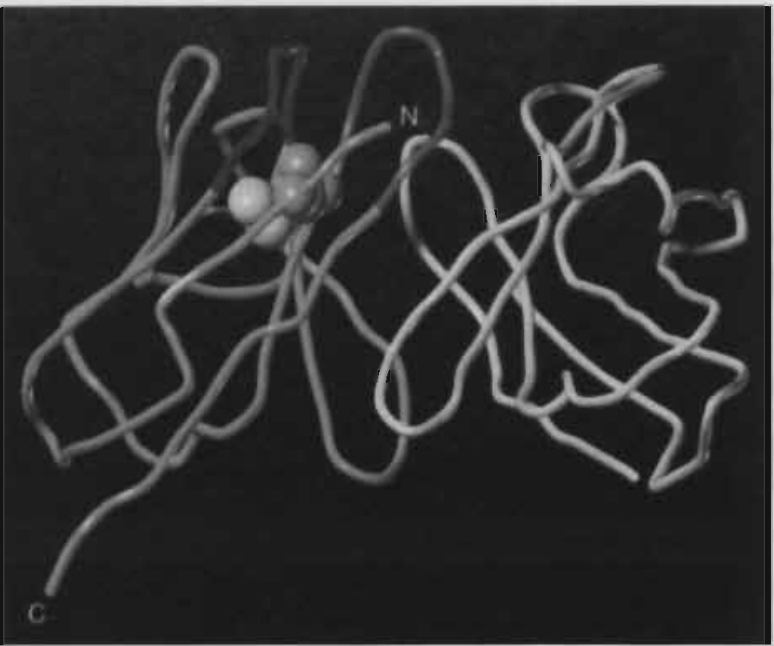

(B)

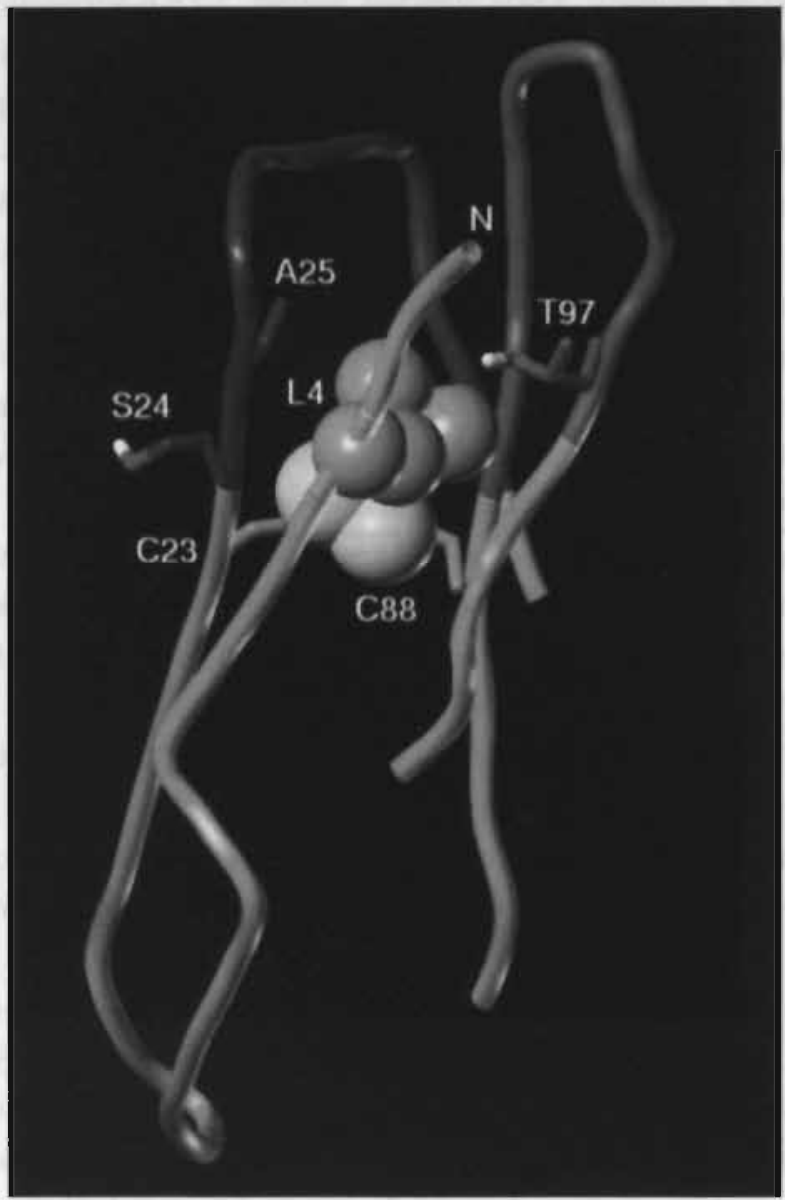

Figure 4. (A) Presentation of the Fy structure of antibody AN02 (PDB 1BAF) made with the program Sybyl (Tripos) showing the location of residue number 4 of $V_{K}$ (leucine in orange) in relation to the intradomain disulfide bond (yellow).

(B) A detailed picture of the position 4 residue of $V K$ (in orange, represented as Vanderwaals spheres), the disulfide bond (yellow, as Vanderwaals spheres) and its neighbouring residues serine 24 , alanine 25 and threonine 97 (in colour backbone, represented as sticks). $\mathrm{C} \alpha$-trace representation of the light (green) and heavy (yellow) chains of the Fv-domain. These images illustrate the buried position of residue 4 between the $\mathrm{N}$ terminus of the $\mathrm{L}$ chain and the amino terminal part of CDR L1 (blue) and the carboxy terminal part of CDR L3 (purple). The oxygen atoms of the side chain groups in serine 24 and threonine 97 are shown in red, the attached hydrogen atoms in white. 
than of clone M4S (clone AB4-M4Smut in Fig. 2C), but in ELISA no clear signals were found (results not shown).

By examination of the structures of related antibodies we identified a number of neighbouring residues, which might be interacting with the mutant residue serine. However, upon grafting in the M4S mutant of AB4 the antigen binding activity, as found for the wild type constructs, could not be restored.

\section{Discussion}

\subsection{VK residue number 4 affects antigen binding}

We demonstrate that the serine residue at position 4 of VL-FR1, which was introduced by cloning site Sall, is responsible for the absence of antigen binding in ELISA of the scFv's derived from antibody $\mathrm{AB} 3$ and $\mathrm{AB} 4$. The Sall restriction site was chosen on the basis of the low frequency of cutting within antibody genes (Chaudhary et al., 1990). The light chain variable regions of $\mathrm{AB} 3$ and $\mathrm{AB} 4$ belong to the subgroup II kappa light chain and have methionine as wild type residue number 4 . In respect to antigen binding, these antibodies do not accept serine, a residue that never occurs on this position in AB-s. However, substitution by leucine did not affect antigen binding as was already found by Benhar and colleagues (Benhar and Pastan, 1995), who showed that the mutant antibody with leucine instead of methionine obtained an increased thermal stability. In AB2, which has a class IV kappa light chain and thereby leucine as position 4 residue, the mutation to serine did not affect antigen binding.

We found a similar effect with the primer encoded position 6 residue of $\mathrm{VH}$. AB-s with a class IIA VH region do not tolerate the mutation of glutamine into glutamic acid, the residue which is mainly found in class IA. However, there is a major difference: the scFv produced by the mutants with serine on position 4 . of $\mathrm{Vk}$ reacted with hCG in surface plasmon resonance analysis although with a low affinity, actually too low for obtaining ELISA signals. Such binding profiles were never obtained with the VH position 6 mutants.

\subsection{Effect on structure}

While the position $6 \mathrm{VH}$ mutants can not adapt the immunoglobulin fold necessary for binding to antigen, the folding of the position $4 \mathrm{VK}$ mutants is probably similar as for the wild type. An intermediate resistance towards irypsin digestion suggests that the introduced serine may cause a local deformation. In this way the canonical fold of L1 can be influenced. Both mutation sensitive antibodies $\mathrm{AB} 3$ and $\mathrm{AB} 4$ have a class 4 loop for $\mathrm{L} 1$, while the loop for $\mathrm{AB} 2$ is different and shows to have some similarity with class 8 (classifications were made with Chothia canonical class assignment software internet: http://www.biochem.ucl.ac.uk/ martin/antibodies.html). L1 on its turn may be directly involved 
in binding or indirectly by influencing the position of the other variable loops. It is remarkable to notice the high degree of homology of the VK domains of AB4 and the 4-4-20 antibody. from which the three dimensional structure has been determined in complex with its antigen fluorescein (Davies et al., 1990). Only eight residues are different: five in CDR1, one in FR2, CDR2 and in FR3 (see Fig. 1). It is interesting that the relatively long CDR1 loop of antibody 44-20 harbours three of the residues involved in binding to the antigen. In analogy with antibody 4-4-20 the light chain CDR1 of the two anti-hCG's AB3 and AB4 might be crucial for binding, explaining the drastic effect of mutation of the position 4 residue into serine.

Examination of the published structures of related antibodies showed that the side chain of residue number 4 is oriented towards the hydrophobic core, as is the case with the previously mentioned position 6 residue of $\mathrm{VH}$. It is positioned against residues 25 and 97 , that are polar in $\mathrm{AB}$-s with methionine or non polar in the tolerant $\mathrm{AB} 2$ with leucine on 4 . Exchange of polar residues in the serine sensitive $A B 4$ by non polar residues of the serine tolerant $A B 2$ did not improve the binding activity. In contrast, when a charged residue (arginine) on position 24 of $\mathrm{AB} 4$ was substituted by a polar residue (threonine), a slower dissociation was found with surface plasmon resonance than for the M4S mutant. However, the improved dissociation behaviour did not result in positive ELISA signals. In spite of a solvent orientation of arginine, it may interact with serine, thereby disturbing the local conformation.

\subsection{Consequences for cloning of $A B S$}

This study clearly showed that the $\mathrm{VH}$ and $\mathrm{VK}_{K}$ consensus sequence specific primers can affect the binding characteristics of the encoded AB-fragments in contrast to what has been published before (Froyen et al., 1995). We confirm their observations, that a number of residues tolerate primer encoded substitutions, but especially the residues on positions 2, 4 and 6 in the amino terminal part of the V-domains might be critical. The side chains of these amino acids, located in the first $\beta$ strand, are oriented toward the hydrophobic core and might therefore interact with the side chains of other residues located in the core region and derived from the $\beta$ strand preceding the CDRI-loop. As was published (Schulz and Schirmer, 1979) the three dimensional structure of a protein is essentially unaffected by mutations of residues, which have solvent exposed side groups, whereas a limited variation is allowed at loci containing residues with the side groups directed towards other parts of the protein. It was already noticed for in particular the residues number 2 and 4 of VL and number 2 of VH that these residues, as constituents of the Vemier zone, may adjust the CDR1 loops and thereby fine tune the fit to antigen (Foote and Winter, 1092).

The particular set of primers, used for amplification, and the application of restriction sites, may mutate residues of the Vernier Zone, and thereby can affect antigen binding of bacterially expressed scFv's or Fab's, due to the introduction of non-tolerated aminoacids in the involved 
framework regions. This finding is of general importance for those scientist, who are working in the field of antibody engineering.

\section{References}

Benhar I. and Pastan I. (1995) Identification of residues that stabilize the single-chain Fv of monoclonal antibodies B3. J. Biol. Chem. 270, 23373-23380.

Bentley G. A., Boulot G., Riottot M. M. and Poljak R. J. (1990) Three-dimensional structure of antidiotope-anti-idiotopc complex. Nature 348, 254- 257.

Birken S., Krichevsky A., O'Connor J., Lustbader J. and Canfield R. (1990) in Glycoprotein Hormones: structure, synthesis and biologic lunction pp. 45-61, Serono Symposia.

Chaudhary V. K., Batra J. K., Gallo M. G., Willingham M. C.. FitzGerald D. J. and Pastan I. (1990) A rapid method of cloning functional variable-region antibody genes in Escherichia coli as single-chain immunotoxins. Proc. Natl. Acad. Sci. U.S.A. 87, 1066-1070.

Chothia C. and Lesk A. M. (1987) Canonical structures for the hypervariable regions of immunoglobulins. J. Mol. Biol. 196, 901-917.

Chothia C., L.esk A. M., Tramontano A., Levitt M., Smith-Gill S. J., Air G., Sheriff S., Padlan E. A., Davics D.. Tulip W. R., et al. (1989) Conformations of immunoglobulin hypervariable regions. Nature 342, 877-883.

Clackson T., Hoogenboom H. R., Griffiths A. D. and Winter G. (1991) Making antibody fragments using phage display libraries. Nature 352, 624-628.

Davies D. R., Padlan E. A. and Sheriff S. (1990) Antibody-antigen complexes. Ann. Rev. Bioch'm. 59, 439-473.

De Haard H. J. W.. Kazemier B., Van der Bent A., Oudshoorn P., Boender P., Van Gemen B., Arends J.-W. and Hoogenboom H. R. M. (1998) Absolute Conservation of Residue number 6 of Immunoglobulin Heavy Chain Variable Regions of class IIA is required for correct folding. Protein Eng., 11, 1267-1276.

Dower W. J., Miller J. F. and Ragsdale C. W. (1988) High efficiency transformation of $E$. coli by high voltage electroporation. Nucleic Acids Res. 16, 6127-6145.

Foote J. and Winter G. (1992) Antibody framework residues affecting the conformation of the hypervariable loops. J. Mol. Biol. 224, 487-499.

Froyen G., Hendrix D., Ronsse I., Fiten P., Martens E. and Billiau A. (1995) Effect of VH and VL consensus sequence-spccitic primers on the binding and neutralizing potential of a single-chain FV directed towards HuIFN-gamma. Mol. linmunol. 32, 515-521.

Glockshuber R.. Schmidt T. and Pluckthun A. (1992) The disulfide bonds in antibody variable: domains: effects on stability: folding in vitro, and functional expression in Escherichia coli. Biochem. 31, 1270-1279. 
Herron J. N., He X. M., Mason M. L., Voss E. W. Jr. and Edmundson A. B. (1.989) Threedimensional structure of a fluorescein-Fab complex crystallized in 2-methyl-2,4pentanediol. Proteins 5, 271-280.

Hopp, T. P., Prickett, K. S., Price, V. L., Libby, R. T., March, C. J., Cerretti, D. P., Urdall, D. L. and Conlon. P. J. (1988) A short polypeptide marker sequence useful for recombinant prulein identitication and purilication. BioTechnol. 6, 1204-1210.

Hormon R. M., Hunt H. D.. Ho S. N., Pullen J. K. and Pease L. R. (198y) Engineering hybrid genes without the use of restriction enzymes: gene splicing by overlap extension. Gene 77 , $61-68$.

Kabat E. A., Wu T. T., Reid-Miller M., Perry H. M. and Gottesman K. S. (1991) Sequences of Proteins of Immunological Interest (DHHS, Washington, DC). 5th Ed.

Leahy D. J., Hynes T. R., McConnell H. M. and Fox R. O. (1988) Crystallization of an anti2,2,6,6-tetramethyl-1-piperidinyloxy-dinitrophenyl monoclonal antibody Fab fragment with and without bound hapten. J. Mol. Biol. 203, 829-830.

Marks J. D., Tristem M., Karpas A. and Winter G (1991) Oligonucleotide primers for polymerase chain reaction amplification of human immunoglobulin variable genes and design of family-specific oligonucleotide probes. Eur. J. Immunol. 21, 985-991.

Marks J. D., Hoogenboom H. R., Bonmert T. P., McCafferty J., Gritfiths A. D. and Winter G. (1991) By-passing immunization. Human antibodies from V-gene libraries displayed on phage. J. Mol. Biol. 222, 581-597.

McCafferty J., Griffiths, A. D., Winter G. and Chiswell D. J. (1990) Phage antibodies: filamentous phage displaying antibody variable domains. Nalure 348, 552-554.

Minsky A., Summers R. G. and Knowles J. R. (1986) Secretion of beta-lactamase into the periplasm of Escherichia coli: evidence for a distinct release step associated with a conformational change. Proc. Natl. Acad. Sci. U.S.A. 83, $4180-4184$.

Orlandi R., Gussow D. H., Jones P. T. and Winter G. (1989) Cloning immunoglobulin variable domains for expression by the polymerase chain reaction. Proc. Natl. Acad. Sci. U.S.A. 86, 3833-3837.

Padlan E. A., Silverton E. W., Sheriff S., Cohen G. H., Smith-Gill S. J. and Davies D. R. (1989) Structure of an antibody-antigen complex: crystal structure of the HyHEL-10 Fab-lysozyme complex. Proc. Natl. Acad. Sci. U.S.A. 86, 5938-5942.

Padlan E. A. (1991) A possible procedure for reducing the immunogenicity of antibody variable domains while preserving their ligand-binding properties. Mol. Immunol. 28, 489-498.

Riechmann L., Clark M., Waldmann H. and Winter G. (1988) Reshaping human antibodies for therapy. Nature 332, 323-327.

Sastry L., Alting-Mees M., Huse W. D., Short J. M., Sorge J. A., Hay B. N., Janda K. D., Benkovic S. J. and Lemer R. A. (1989) Cloning of the immunological repertoire in 
Escherichia coli for generation of monoclonal catalytic antibodies: construction of a heavy chain variable region-specific cDNA library. Proc. Natl. Acad. Sci. U.S.A. 86, 5728-5732.

Schulz, G. E. and Schirmer R. H. (1979) Principles of Protein Structure, Springer-Verlag, New York.

Sheriff S., Silverton E. W., Padlan E. A., Cohen G. H., Smith-Gill S. J., Finzel B.C. and Davies D. R. (1987) Three-dimensional structure of an antibody-antigen complex. Proc. Natl. Acad. Sci. U.S.A. 84, 8075-8079.

Skerra A. and Plückthun A. (1991) Secretion and in vivo folding of the Fab fragment of the antibody McPC603 in Escherichia coli: influence of disulphides and cis-prolines. Protein Eng. 4, 971-979.

Tramontano A., Chothia C. and Lesk A. M. (1990) Framework residue 71 is a major determinant of the position and conformation of the second hypervariable region in the VH domains of immunoglobulins. J. Mol. Biol. 215, 175-182.

\section{Acknowledgements}

We wish to express our gratitude to Bert van der Wijer (Organon Teknika) for the generous gift of purified hCG, to Peter van Weezenbeek (Organon) for the expression vector $\mathrm{pVI}$, and to Hennie Hoogenboom and Ivo Horn (CESAME at the University Hospital Maastricht) for critical reading of the manuscript. This work was supported by PBTS grant BIO 91028 from the Dutch Ministry of Economic Affairs. 



\section{ChAPTER 3}

\section{QUANTIFICATION OF SINGLE-CHAIN FV'S WITH SURFACE PLASMON RESONANCE}

Published as

Determination of active single chain antibody concentrations in crude periplasmic fractions

Bert Kazemier, Hans J. W. de Haard, Piet Boender, Bob van Gemen, and Hennie R.J.M. Hoogenboom

Journal of Immunological Methods

1996; Volume 194, No. 2; pages 201 - 209 


\section{Abstract}

We have measured active single chain antibody ( $\mathrm{scFv}$ ) concentration under mass transfer limitation conditions using surface plasmon resonance on the BIAcore. For the creation of a standard curve scFv 4Dwt, derived from monoclonal antibody (mAb) 4D, directed against human chorionic gonadotropin (hCG), was purified by positive affinity chromatography. Determination of the active antibody fraction after purification was performed using antiFLAG, reactive against a tag sequence C-terminally fused to the scFv. Two independent experiments showed that the activity remaining represented over $75 \%$ of the total amount of purified protein. Calibration curves on high density antigen surfaces showed a linear relationship between antibody concentration and binding rates. Periplasmatic fractions of six mutant scFv's. also derived from mAb 4D, revealed a clear difference in the amount of soluble active scFv expressed in the periplasm of $E$. coli compared with the total amount of antibody present, indicating the necessity of measuring active antibody concentrations. This rapid concentration determination method will be particularly useful for accurately comparing affinity constants, using antibody concentrations determined with the BIAcore, of the many different scFv fragments routinely isolated from phage display libraries.

\section{Introduction}

Phage display has become an increasingly important tool in biotechnology. By expressing the variable regions of antibodies (as single chain antibodies (Huston et al., 1988, Bird et al., 1988)) as geneIII fusion proteins on the tip of the filamentous phage MI3 (McCafferty et al., 1990), it is possible to select antigen binding phage, exposing the appropriate recombinant antibody, from a large pool of non-binders (Clackson et al., 1991; Hoogenboom and Winter, 1992; Barbas et al., 1993; Griffiths et al., 1993). The method is very powerful: many different antibodies can be generated against the same antigen (Marks et al., 1991: Griffiths et al., 1993), making the availability of a fast and accurate affinity screening assay to identify the best antibody fragment a crucial step. The best screening assays use unpurified antibodies (crude $E$. coli supernatant of periplasmatic fractions) and are sensitive and robust. So far screening methods to determine the $K_{\mathrm{eq}}$ by ELISA (Friguet et al., 1985) or the off-rate, using the BIAcore (Hawkins et al., 1992) have been shown to be effective. To determine the affinity constant of an antibody-antigen interaction, it is essential to know the amount of scFv fragments in the crude preparation. In the present study we describe a rapid system for the determination of the amount of soluble reactive scFv, expressed in the periplasm of $E$. coli. usins surface plasmon resonance (Liedberg and Lundstrom, 1993) on the BIAcore (Karlsson et al., 199l) in comparison with the total amount of scFv present, estimated from immunoblots. Mab 4D, directed against hCG 
(Birken et al., 1990), was used as a model. Several mutants and the wild type scFv, derived from $\mathrm{mAb} 4 \mathrm{D}$, were created. The influence of mutations in oligo encoded framework regions was examined on both reactivity and expression level.

\subsection{Theoretical background}

A mass transport limitation situation was created by immobilizing a large amount of antigen on the surface of a sensor chip (Glaser, 1993; Karlsson et al.,1993; 1994). In this situation mass increase on the surface of a sensor chip becomes dependent only on antibody concentration. In BlAcore the rate of complex formation may be explained in terms of mass transfer of the analyte to the surface and in terms of analyte-ligand reaction kinetics (Eq. 1).

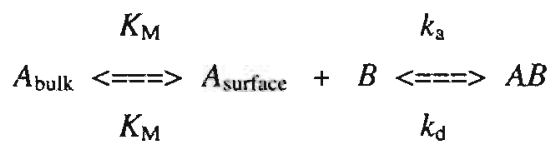

where $A$ is the analyte, $B$ is the ligand, $K_{\mathrm{M}}$ is the mass transport coefficient and $k_{\mathrm{a}}$ and $k_{\mathrm{d}}$ are the association and dissociation rate constants respectively. The mass transport coefficient is dependent on the diffusion coefficient and therefore depends on the molecular weight of the analyte. A high association constant and high amounts of immobilized ligand will cause the switch from $A_{\text {bulk }}$ to $A_{\text {surface }}$ to be the rate limiting step and therefore the overall reaction will be limited by mass transfer. For a standard one to one reaction between analyte and ligand the rate of complex formation can be described as:

$$
\mathrm{d}[\mathrm{AB}] / \mathrm{d} t=k_{\mathrm{a}}[\mathrm{A}][\mathrm{B}]-k_{\mathrm{d}}[\mathrm{AB}]
$$

Rewritten in terms of BIAcore response :

$$
\mathrm{dRU} / \mathrm{d} t=k_{\mathrm{a}} C R_{\max }-\left(k_{\mathrm{a}} C+k_{\mathrm{d}}\right) \mathrm{RU}
$$

where RU is the detector response, $C$ is the analyte concentration and $R_{\max }$ is the maximum amount of analyte that can be bound by the ligand (O'Shannessy et al., 1993). Under mass transport limitation, on a high density surface, we can neglect the dissociation term giving a situation where the rate of complex formation is constant (Eq. 4).

$$
\mathrm{dRU} / \mathrm{d} t=k_{\mathrm{a}} C R_{\max }
$$

The initial binding rate will be independent of $k_{\mathrm{a}}$ and merely dependent on analyte concentration. From the sensorgrams of different known analyte concentrations the binding rates 
can be determined and plotted against the concentration yielding a standard curve from which active concentrations of unknown samples can be calculated.

\section{Materials and methods}

\subsection{Affinity purification of scFv 4Dwt}

The wild type scFv derived from anti-hCG mAb 4D, coded 4Dwt, was purified from total cell lysate according to Wells (Tumor Biology Center, Freiburg, Germany, personal communication). Briefly, 1 litre of 2 TY Amp Glu medium was inoculated (1:100) with overnight culture and grown to $\mathrm{OD}_{600}=0.5$ at $37^{\circ} \mathrm{C}$. The cells were pelleted and resuspended in 1 litre of 2 TY Amp IPTG ( $1 \mathrm{mM}$ final concentration). The culture was grown for another 2.5 hours at room temperature. The cells were harvested and lysed in 1/100 of the original volume of phosphate buffered saline (PBS); $8 \mathrm{M}$ urea. After centrifugation the supernatant containing the denatured proteins was renaturated by dialysis against PBS. Renaturated protein was incubated batchwise with hCG-Sepharose (hCG, from urine of pregnant women purified on sephacryl S200 HR, coupled to NHS activated Sepharose; approximately $1.5 \mathrm{mg}$ hCG/600 mg Sepharose, wet weight). After overnight binding $\left(4^{\circ} \mathrm{C}\right)$ and extensive successive washings with $\mathrm{PBS}, 50 \mathrm{mM}$ Tris/Cl pH 7.5; $0.5 \mathrm{M} \mathrm{NaCl}$ and $50 \mathrm{mM}$ Tris/Cl pH 9.0;0.5 M NaCl, the antibody was eluted with $0.1 \mathrm{M}$ triethylamine and immediately neutralized by the addition of $0.5 \mathrm{vol} 1 \mathrm{M} \mathrm{Tris} / \mathrm{Cl} \mathrm{pH}$ 7.0. The eluate was dialyzed against HBS (10 mM Hepes $\mathrm{pH} 7.4 ; 0.15 \mathrm{M} \mathrm{NaCl} ; 3.4 \mathrm{mM}$ EDTA; $0.05 \% \mathrm{P} 20$ ). The amount of purified $\mathrm{s} F \mathrm{Fv}$ was measured spectrophotometrically (assuming that 1 $\mathrm{OD}_{280}=0.7 \mathrm{mg} / \mathrm{ml}$; Marks et al., 1991) and by micro-BCA (Pierce, according to the manufacturer's manual), as a double check. Purity was checked on SDS-PAGE followed by Coomassie Brilliant Blue staining.

Table 1. Oligo encoded $\mathrm{N}$-terminal amino acid differences of the analyzed mutants.

\begin{tabular}{|c|c|c|c|c|c|c|c|c|c|c|c|c|c|c|c|}
\hline \multirow{2}{*}{$\begin{array}{c}\text { clone } \\
\text { 4D21 (wt) }\end{array}$} & \multicolumn{8}{|c|}{ VH-FR1 } & \multicolumn{7}{|c|}{ VL-FR1 } \\
\hline & $Q$ & V & $Q$ & L & $Q$ & $Q$ & $S$ & G & D & V & $\mathrm{V} M$ & $\mathrm{~T}$ & $Q$ & $\mathrm{~T}$ & $\mathrm{P}$ \\
\hline 4D11 & - & - & - & - & - & $E$ & - & - & - & $I$ & $-S$ & - & - & - & - \\
\hline $4 \mathrm{DI} 5$ & - & - & - & - & - & - & - & - & - & I & $-S$ & - & - & - & - \\
\hline $4 \mathrm{D} 16$ & $\mathrm{~N}$ & $I$ & - & - & E & - & - & - & $\mathrm{K}$ & - & $-\mathrm{L}$ & - & - & $S$ & - \\
\hline $4 \mathrm{D} 17$ & - & - & - & - & - & - & - & - & $\mathrm{E}$ & - & $-L$ & - & - & $S$ & - \\
\hline $4 \mathrm{D} 200$ & - & - & - & - & - & - & - & - & - & - & $-S$ & - & - & - & - \\
\hline
\end{tabular}




\subsection{Sample preparation}

Periplasmatic fractions of six mutant clones derived from mAb 4D, including the wild type scFv coded 4D21, were prepared as follows (Table 1 shows the different mutant sequences). Two different rounds of growth and induction were perfonned. After growth $(50 \mathrm{ml} \mathrm{scale})$ and induction, as described above, cells were harvested and resuspended in ice cold BBS $(0.2 \mathrm{M}$ borate $\mathrm{pH} 8.0 ; 150 \mathrm{mM} \mathrm{NaCl} ; 1 \mathrm{mM}$ EDTA). The resuspension volume was $1 / 100$ of the original volume $\mathrm{X} \mathrm{OD} 600$, at the time of harvesting, thereby correcting for growth rate differences. After $1 \mathrm{~h}$ of head over head rotation $\left(4^{\circ} \mathrm{C}\right)$ the cells were pelleted and the supernatant (periplasmatic fraction, PPF) was tested in the BIAcore.

\subsection{Equipment and reagents}

The BIAcore system, sensor chip CM5, surfactant P20 and amine coupling kit were purchased from Pharmacia Biosensor, Uppsala Sweden. MAb anti-FLAG (M2) was obtained from Eastman Kodak Company, New Haven, USA. The BCA protein assuy reagent (microBCA) was obtained from Pierce, Rockford, USA. "Ultra free" microcentrifuge filter units $(0.22$ $\mu \mathrm{M})$ were purchased from Millipore, Bedford MA, USA. ECL immunoblot detection reagent was obtained from Amersham, Bucks, UK and RAM-HRP was obtained from DAKO-Patts, Glostrup, Denmark.

\subsection{Immobilization}

Coupling of anti-FLAG or hCG to a sensor chip CM5 was performed as previously described by Löfas and Johnsson, (1990). The carboxylated dextran matrix was activated by injection of $48 \mu] 0.2 \mathrm{M} \mathrm{N}$-ethyl-N'-(dimethylaminopropyl)carbodiimide (EDC) / $0.05 \mathrm{M} \mathrm{N}$ hydroxysuccinimide (NHS) at a flow rate of $3 \mu \mathrm{l} / \mathrm{min}$. $48 \mu \mathrm{l}$ anti-FLAG was injected $(100 \mu \mathrm{g} / \mathrm{ml}$ in $10 \mathrm{mM}$ sodiumacetate $\mathrm{pH} 5.5)$. Using a flow rate of $5 \mu \mathrm{l} / \mathrm{min}$ hCG $(45 \mu \mathrm{l}$ at $150 \mu \mathrm{g} / \mathrm{ml}$ in 10 $\mathrm{mM}$ sodiumacetate $\mathrm{pH} 5.0$ ) was injected in a flow cell that was activated with $45 \mu \mathrm{l}$ EDC/NHS. Residual activated groups were blocked by injection of $35 \mu l$ ethanolamine (1.0 M pH 8.5). Noncovalently bound protein was removed by a regeneration procedure $(10 \mathrm{mM} \mathrm{HCl} ; 5 \mu$ injection, three times) prior to injection of the samples.

\subsection{Determination of the activity determination of purified scFv $4 D w t$}

Two concentrations of the purified scFv 4Dwt ( $200 \mu \mathrm{l}$ volumes, 200 and $300 \mathrm{nM})$ were incubated with hCG-Sepharose (the same material as used for the purification of the scFv) and $3 \mathrm{~A}-$ Sepharose (a non-binding control containing mAb $3 \mathrm{~A}$, directed against hCG, coupled to the Sepharose). After overnight binding the supernatants were separated from the Sepharose using a $0.2 \mu \mathrm{M}$ microfuge filter. Protein concentrations were determined with micro-BCA (Pierce) and the supernatants were tested in BIAcore for their response on an anti-FLAG surface. 
Residual reactivity was also tested directly in the BIAcore by injecting $20 \mu \mathrm{l} \mathrm{scFv} 4 \mathrm{Dwt}$ diluted in HBS $(300,200,150,1(10,75$ and $50 \mathrm{nM}$, flow $5 \mu 1 / \mathrm{min})$ on anti-FLAG surface, followed by a constant $h C G$ injection $(10 \mu l, 15 \mu \mathrm{g} / \mathrm{ml})$.

\subsection{Concentration determination using the BIAcore}

For the standard curves, $20 \mu \mathrm{l}$, at a flow rate of $5 \mu \mathrm{l} / \mathrm{min}$, of the purified 4Dwt was injected $(80,60,40,30,20,10 \mathrm{nM}$, active concentrations) into a flow cell to which high levels of hCG were inmobilized. Of the mutant-PPF's three dilutions (1:5, 1:10, 1:20, $20 \mu 1$ each) were injected.

\subsection{Concentration determination using immunoblots}

Periplasmatic fractions were run on standard reducing SDS-PAGE gels. After blotting on nitrocellulose, the filters were blocked with non-fat dry milk and incubated with anti-FLAG followed by RAM-HRP and ECL-detection.

\section{Results}

\section{I Determination of active scFv concentration}

When analysing the kinetics of a reaction the correct analyte concentration is essential for determination of the association rate constant. The presence of small amounts of inactive antibody (especially with bivalent antibodies) can have a major influence on the determination of the affinity constant (Azimzadeh and Van Regenmortel, 1991). After purification of scFv 4Dwt the protein concentration was determined in two ways. Both $\mathrm{OD}_{280}$ measurement and micro-

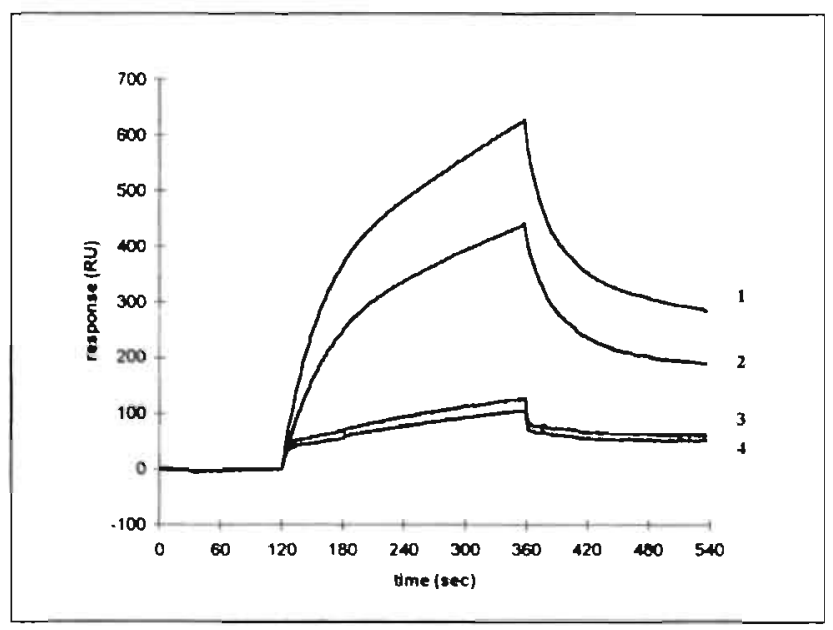

Figure 1. Overlay plot of ScFv 4Dwt captured on anti-FLAG after binding to antigen (sensorgrams $\# 3,300 \mathrm{nM}$ and $\# 4,200 \mathrm{nM}$ ) and on a nonbinding control (sensorgrams \#1,300 $\mathrm{nM}$ and $\# 2,200 \mathrm{nM}$ ). The sample was injected at $t=120 \mathrm{~s}$. Dissociation (buffer wash) started at $\mathrm{t}=360 \mathrm{~s}$. From this dissociation phase the remaining reactivity was calculated (see text for details). 
BCA yielded the same concentration. SDS-PAGE followed by Coomassie Brilliant Blue staining of the purified sample showed no evidence of contaminating proteins (data not shown).

Since the high $\mathrm{pH}$ elution step used in purification can inactivate the scFv, it was necessary to determine the active proportion of the total amount of purified scFv 4Dwt. Two procedures were used for measuring the reactivity of $s c F v 4 D w t$. First. 4Dwt was captured on hCGSepharose and on 3A-Sepharose (non binding control). Protein concentration determination of $4 \mathrm{Dwt}$ supernatants, pre and post adsorption to antigen, showed a residual reactivity of $79 \%$ ( \pm $4 \%$ ) as determined with the micro-BCA. Supernatants from the binding experiments were screened on an anti-FLAG surface. The response obtained from the supernatants after overnight binding on 3A-Sepharose is referred to as the total response. The response from the supernatants after binding 4Dwt on hCG-Sepharose was obtained from non-bound inactive material. The immunoreactive fraction was determined from the dissociation phase $(360-540 \mathrm{~s})$ of the sensorgrams by calculating, every $5 \mathrm{~s}$, the RU values from the non-bound response (Fig. I, sensorgrams \#3 and \#4) relative to the RU values from the total response (Fig. 1, sensorgrams \# 1 and \#2). RU values were determined from the dissociation phase since the response during the injection phase is influenced by the bulk refractive index of the different samples. The remaining reactivity proved to be $80 \%$ ( $\pm 5 \%$ ), which is comparable to the values obtained with micro-

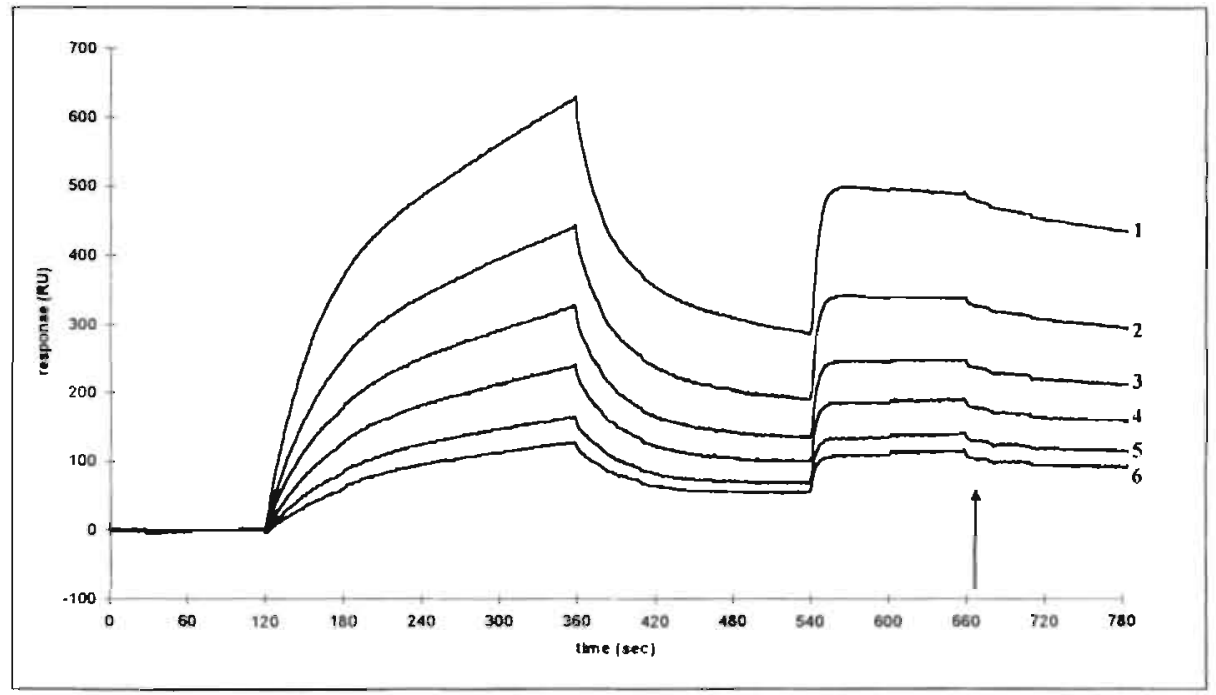

Figure 2. Typical overlay plot of activity determination of scFv 4Dwt on anti-FLAG. Incteasing scFv concentrations were injected at $t=120$ s (sensorgrams: \#1, $300 \mathrm{nM} ; \# 2,200 \mathrm{nM} ; \# 3,150 \mathrm{nM}: \# 4,100$ $\mathrm{nM}: \# 5.75 \mathrm{nM}$ : \#6, $50 \mathrm{nM}$ ) followed by a constant $\mathrm{hCG}$ injection at $t=540 \mathrm{~s}$. RU values for calculating the immunoreactive fraction were determined at the point indicated by the arrow $(t=670 \mathrm{~s})$.

BCA (see above). The supernatants obtained after ovemight binding on hCG-Sepharose were also tested on an hCG surface in the BlAcore and showed no detectabie signal indicating that all 
active ScFv molecules had bound to the hCG-Sepharose and that the response of this supernatant on anti-FLAG must have been associated with inactive molecules (data not shown). The responses, on anti-FLAG (Fig. 1, sensorgrams 1 and 2), of the supernatants after binding to 3ASepharose were the same as from the starting material (Fig. 2, sensorgrams 1 and 2) indicating that there was no non-specific binding to the 3A-Sepharose (overlay not shown).

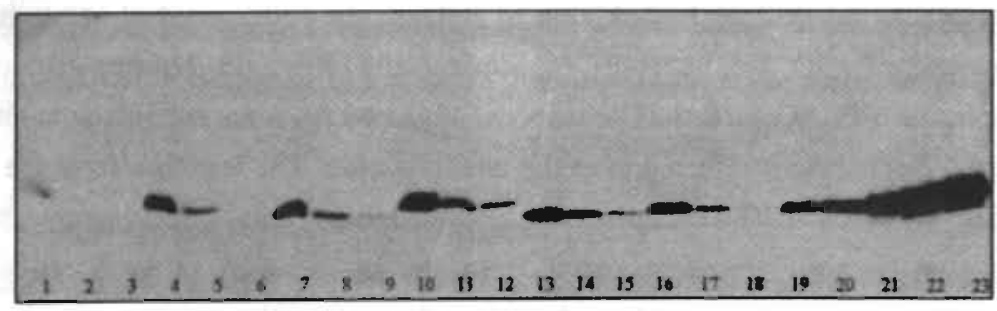

Figure 3. Immunoblot ECL development of a typical immunoblot experiment of the second periplasmic extraction. The amount of PPF, or purified scFv, loaded on to the gel is indicated below. Lanes 1-3:

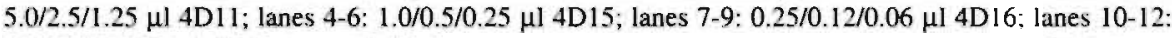

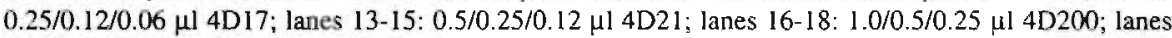
19-23: $5 / 10 / 20 / 40 / 80 \mathrm{ng}$ purified $4 \mathrm{Dwt}$.

Second, 4Dwt was injected on to an anti-FLAG surface using different concentrations, followed by an hCG injection with a constant concentration (Fig. 2). The immuno reactive fraction was determined at a single time point, $t=670 \mathrm{~s}$. At this point the responses were high and there was no contribution of the bulk refractive index to the signal. The response of 4Dwt was corrected by extrapolating the response at $t=540 \mathrm{~s}$ to $t=670 \mathrm{~s}$, using the dissociation rate constant calculated from the time frame $(t=500-540 \mathrm{~s})$ just before hCG injection. On runs with different amounts of scFv, using Eq. (5), the ratio of bound hCG relative to the amount of bound scFv shows that $75 \%( \pm 3 \%)$ of the purified 4 Dwt was active.

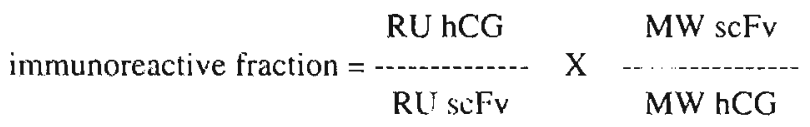

\subsection{Concentration determination of $s c F v ' s$}

Total antibody concentrations were determined from immunoblots as shown in Fig. 3. Sample concentrations were estimated from known marker concentrations run on the same gel and denoted in Table 2.

For the analysis of active antibody concentration in crude extracts, purified scFv 4Dwt was used to create a standard curve. 4Dwt was injected in serial dilutions on to a sensor chip on which hCG (2850 RU) was immobilized. This surface proved to be extremely stable since over 50 runs, including efficient and complete $\mathrm{HCl}$ regeneration, could be performed with less than $10 \%$ loss 
of surface reactivity (data not shown). Overlay plots of both the normal and the deduced (In $(\mathrm{dRU} / \mathrm{d} t$ ) vs. $t$ ) sensorgrams, showing a horizontal straight line at constant binding rates, revealed

Table 2. Active antibody concentrations, calculated from the slopes of the sensorgrams, and total antibody concentrations determined by immunoblotting, of six different mutants after two independent growth/induction rounds.

\begin{tabular}{|c|c|c|c|c|}
\hline \multirow[t]{3}{*}{ Mutant } & \multicolumn{4}{|c|}{ scFv concentration $( \pm S D n M)$} \\
\hline & $\begin{array}{l}\text { 1st periplasmic } \\
\text { extraction }\end{array}$ & & $\begin{array}{l}\text { 2nd periplasmic } \\
\text { extraction }\end{array}$ & \\
\hline & Total & Active & Total & Active \\
\hline 4D11 & $40 \pm 20$ & 0 & $50 \pm 25$ & 0 \\
\hline $4 \mathrm{D} 15$ & $200 \pm 100$ & $44 \pm 12$ & $300 \pm 200$ & $32 \pm 12$ \\
\hline $4 \mathrm{D} 16$ & $400 \pm 200$ & $255 \pm 11$ & $1000 \pm 300$ & $471 \pm 20$ \\
\hline 4D17 & $800 \pm 200$ & $498 \pm 11$ & $2000 \pm 350$ & $777 \pm 9$ \\
\hline $4 \mathrm{D} 21$ (wt) & $200 \pm 130$ & $182 \pm 10$ & $800 \pm 250$ & $329 \pm 16$ \\
\hline 4D200 & $100 \pm 50$ & $36 \pm 13$ & $150 \pm 20$ & $34 \pm 12$ \\
\hline
\end{tabular}

that the reaction is indeed mass transfer limited. It can be seen from Fig. 4 that binding rates are constant throughout the injection time. Only for the run with the highest concentration, (Fig. 4A, $80 \mathrm{nM}$ ), the binding rate decreases at the end of the injection phase due to the fact that too many binding sites become occupied and mass transport limitation was abolished. Calculations of the binding rates $\left(k_{\mathrm{s}}\right)$ were performed by choosing an area where the $\ln (\mathrm{dRU} / \mathrm{d} t)$ vs. $t$ is constant (200-230 s), using the line fit command from the BlA-evaluation menu. The binding rates $\left(k_{\mathrm{s}}\right)$ were plotted against the concentration of 4Dw' (Fig. 4C). From this calibration curve unknown active antibody concentrations could be determined. Crude periplasmatic fractions of six mutants from two independent growth and extraction experiments were injected on the same high density hCG surface. Fig. 5 shows an overlay plot of the sensorgrams of different mutant clones. One mutant expressing non-reactive scFv (coded 4D11) shows a block signal duc to a change in refractive index of the sample but no mass increase (although there is scFv prest:m, as could be determined on the anti-FLAG-surface, data not shown, and on immunoblot, Fig. 3). Differences in the amount of active soluble periplasmic expressed scFv are clearly seen in Fig. 5 . Clones 4DI6 and 4D17, examples of high expression, derived from a phagre library contained more active scFv than the wild type 4D21 and the other mutants. Compared with other reactive clones, mutant 4D200 and 4DI5 showed evidence of a much higher off-rate. Table 2 summarizes the active scFy concentrations of the different mutants. 


\section{Discussion}

A fast and reliable method for antibody concentration determination is of extreme importance when accurate affinity constants have to be determined, especially in the case of selection of antibodies with closely related affinities (e.g. selection of affinity maturated scFvs from a phage display library, Hawkins et al., 1992). Time consuming purifications should be avoided and this is where surface plasmon resonance offers a possible solution.

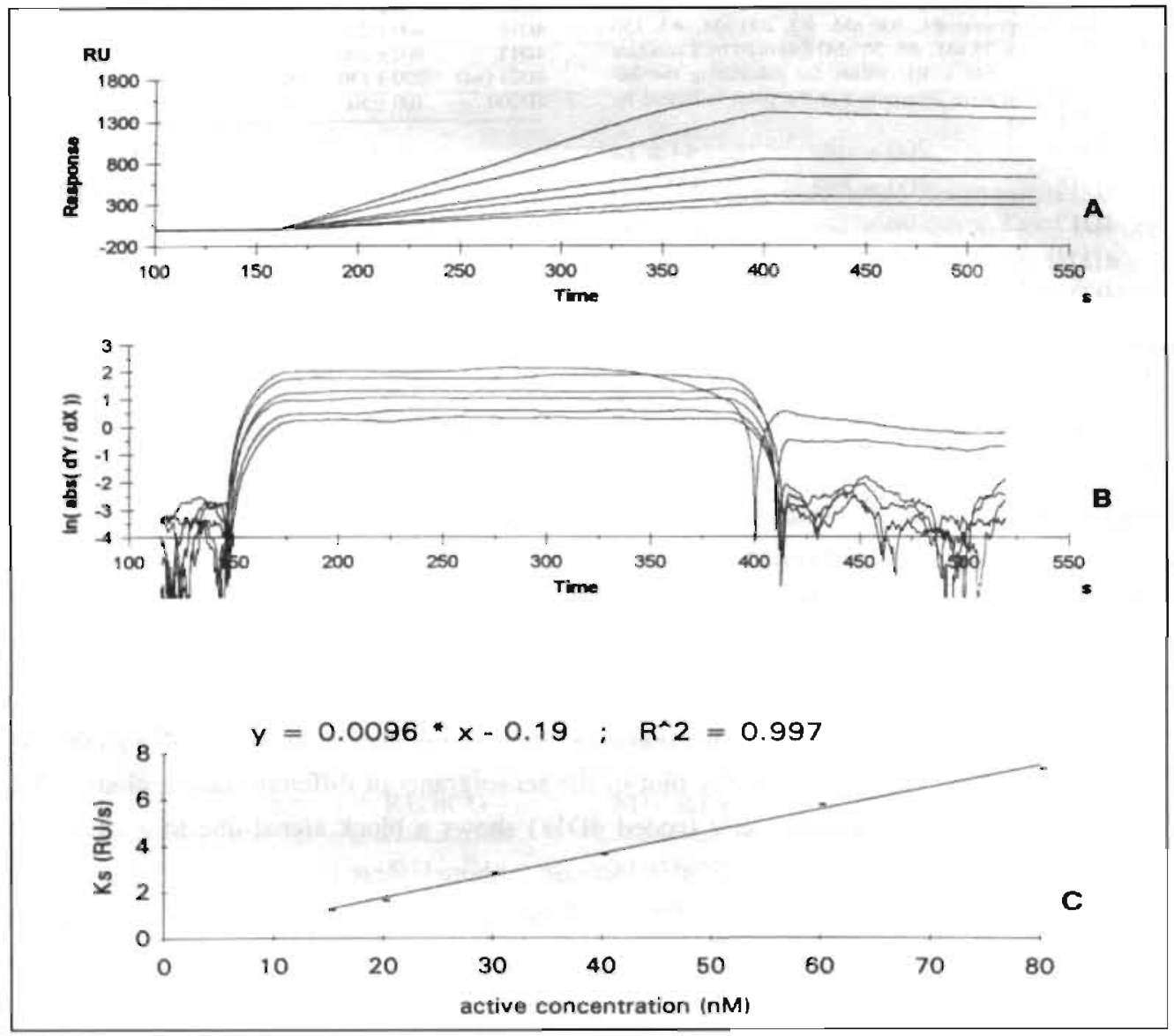

Figure 4. Standard curve of scFv 4Dwt on hCG. A: increasing concentrations of 4Dwt were injected onto a high density antigen surface (from top to bottom: $80,60,40,30,20$ and $15 \mathrm{nM}$ respectively). $B$ : deduced sensorgrams $(\ln (\mathrm{dRU} / \mathrm{d} t)$ vs $t)$ of Fig. 3A. C: plot of the slope $K_{\mathrm{S}}(\mathrm{RU} / \mathrm{s})$ as a function of the active 4Dwt concentration ( $\mathrm{nM}$ ). 
In order to set up a generic method for measuring total scFv concentration, mAb anti-FLAG, directed against an eight amino acid epitope fused to the $\mathrm{C}$-terminus of the scFv, was used. Over 15000 RUs of mAb anti-FLAG were immobilized on the surface of a sensor chip in order to achieve a mass transport limitation. However. from both Fig. 2 (association phase of scFv 4Dwt on anti-FLAG, $t=120-360 \mathrm{~s}$ ) and from the calculated limit coefficient, $k_{\mathrm{a}}[\mathrm{B}] / K_{\mathrm{M}}$, it is clear that binding rates of scFv on the anti-FLAG are not limited by mass transport. According to Karlsson et al. (1994), the limit coefficient can be used to distinguish between mass transport limited and interaction controlled kinetics. For the binding of scFv 4Dwt to anti-FLAG the limit coefficient, $k_{\mathrm{it}}[\mathrm{B}] / K \mathrm{M} \approx 0.1$, is much smaller than 1 , indicating that the reaction rate is much higher than the mass transport rate, yielding true interaction controlled kinetics.

An altemative method for measuring scFv concentration uses a high density antigen surface and requires a well known standard. During the course of this work Abraham et al. (1995) reported a method for measuring the approximate concentration of scFv, Fab, diabody and $\operatorname{IgG}$ in unpurified samples by mass transport limited binding on high density antigen surfaces on the BIAcore. One of their conclusions was that a 'gold standard' had to be created. In this study we describe the creation of the so called 'gold standard'. Two different BLAcore binding studies revealed comparable reactivity percentages of the purified scFv $4 \mathrm{Dwt}$. One flaw is that for the Sepharose binding experiments very high concentrations of purified $s c F v$ are required to obtain a signal above the noise level on an anti-FLAG surface in the case of the non-reactive fraction (Fig. 1). In this case $300 \mathrm{nM}$ total scFv 4 Dwt containing $60 \mathrm{nM}$ inactive scFv gave a response of less than $100 \mathrm{RU}$. Another drawback of this method is the difference in dissociation rate between samples with high and low analyte concentration. Samples with a high scFv concentration (3ASepharose supernatants) have a much higher initial dissociation rate than samples with a low scFv concentration. This can be explained by the fact that with low analyte concentration present on high density surface rebinding can occur leading to a lower dissociation rate. This difference

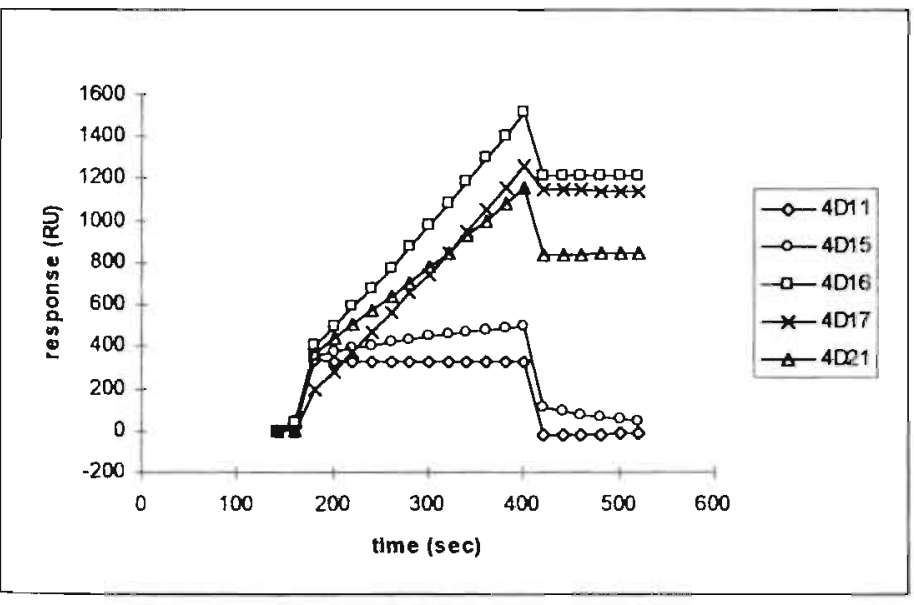

Figure 5. Sensorgrams of mutants and the wild type (4D21) scFvs derived from mAb $4 D$ injected onto a high density hCG surface. The periplasmic fractions were diluted $1 / 5$ in HBS, except clone 4D17 which was diluted $1 / 10$ in HBS. 
in dissociation rate results in a relatively high standard error. In the first part $(t=360-420 \mathrm{~s})$ of the dissociation phase the amount of total scFv bound on anti-FLAG is higher (Fig. 1, sensorgrams \#1 and \#2) relative to the amount of inactive bound scFv (Fig. 1, sensorgrams \#3 and \#4) as compared with the end part of the dissociation phase ( $t=420-540 \mathrm{~s})$. The reactivity determination by sequential injections of $\mathrm{scFv}$ followed by hCG on the anti-FLAG surface on the BIAcore, as shown in Fig. 2, is preferred because of its speed, sensitivity and reproducibility.

The purified scFv 4Dwt was used to create a standard curve on a high density hCG surface. From Fig. $4 \mathrm{C}$ it can be concluded that there is a clear linear relationship between antibody concentration and binding rate indicating that under mass transport limitation conditions an increase of mass on the surface is only concentration dependent. Two independent experiments showed that the standard curves were identical (statistical data not shown). By using this calibration curve we were able to determine active $s c F v$ concentrations expressed in the periplasm of different mutant scFvs derived from mAb 4D (as described in Table 1) and we compared these with the total concentrations. Two independent growth and induction rounds (Table 2) show that the expression levels are highest for the phage derived clones 4DI6 and $4 \mathrm{D} 17$, and for the wild type construct $4 \mathrm{D} 21$ and that the active fraction is about the same as (4D21, 1st extraction) to one third (4DI7, 2nd extraction) of the total amount of scFv present. Mutant clones 4D15 and 4D200 show similar expression levels of which one third (4D200, 1st extraction) to only one tenth (4DI5, 2nd extraction) is active. These clones also display a high off-rate which might be the reason why these mutants do not yield positive results in an ELISA (during washing and incubation steps the scFv is probably washed out leading to an ELISAsignal that does not exceed the background, data not shown). Mutant 4D11 contained no detectable reactive $\mathrm{scFv}$ although there is a clear amount of scFv present on immunoblot. Although there is some variation in the amount of active scFv produced by a single mutant in independent extractions, a clear difference in expression level of total and active scFv between different mutants can be seen.

Our conclusion is that surface plasmon resonance under mass transport limitations, by using large amounts of immobilized antigen. is a very useful and sensitive method for the rapid measurement of active single chain antibody concentration in crude extracts which avoids the need for sample purification. Comparison of active and total concentrations underline the necessity of measuring active concentrations since up to $90 \%$ of the total amount of protein present may be inactive. A drawback of the BIAcore method is that only concentrations of scFvs with high association constants, $K_{a}>1 \times 10^{5} 1 / \mathrm{Ms}$, can be measured. Lower $K_{a}$ s,$<1 \times 10^{5}$, do not yield mass transport limited association and therefore cannot be measured using the BIAcore approach. The creation of a generic method for determining total scFv concentration, on antiFLAG under mass transport limitation conditions, proved to be impossible. This is due to the relatively low association constant, $4 \times 10^{4} 1 / \mathrm{Ms}$, of the anti-FLAG for scFvs leading to the 
necessity of immobilizing over 150000 RUs of anti-FLAG (which is about five-fold higher than the detection limit of the system) in order to achieve mass transport limitation situation.

\section{References}

Abraham, R.., Buxbaum, S., Link, J., Smith, R., Venti, C. and Darsley, M. (1995) Screening and kinetic analysis of recombinant anti-CEA antibody fragments. J Immunol. Methods 183, 119.

Azimzadeh, A. and Van Regenmortel, M.H. (1991) Measurement of affinity of viral monoclonal antibodies by ELISA titration of free antibody in equilibrium mixtures. J. Immunol. Methods 141, 199.

Barbas, C.F., Collet, T.A., Amberg, W., Roben, P., Binley, J.M.. Huekstra. D.. Cababa. D., Jones, T.M., Williamson, R.A., Pilkington, G.R., et al. (1993) Molecular prolile of an antibody response to HIV-1 as probed by combinatorial libraries. J. Mol. Biol. 230, 812.

Bird, R.E., Hardman, K.D., Jacobson, J.W., Johnson, S., Kaufmann, B.M., Lee, S.. Lee. T., Pope, S.H., Riordan, G.S. and Whitlow, M. (1988) Single chain antigen binding proteins. Science 242, 423.

Birken, S., Krichevsky, A., O'Connor, J., Lustbader, J. and Canfield, R. (1990) Chemistry and Immunochemistry of hCG, Its Subunits and Its Fragments. In: Chin, W. and Boime, I. (Eds.), Glycoprotein Hormones, Structure, Synthesis and Biologic Function. Serono Symposia, USA, Norwell, Massachusctts, p. 45.

Clackson, T., Hoogenboom, H.R., Griffiths, A.D. and Winter, G. (1991) Making antibody fragments using phage display libraries. Nature 352, 624.

Friguet, B., Chafotte, A.F., Djavadi-Ohaniance, L., and Goldberg, M.E., (1985) Measurement of true affinity constant in solution of antigen-antibody complexes by enzyme linked immunosorbent assay. J. Immunol. Methods 77, 305.

Glaser, R.W. (1993) Antigen-antibody binding and mass transport by convection and diffusion to a surface: a two-dimensional computer model of binding and dissociation kinetics. Anal. Biochem. 213, 152.

Griffiths, A.D., Malmqvist, M., Marks, J.D., Bye, J.M., Embleton, M.J., McCafferty, J., Baier, M.. Holliger, K.P., Gorick. B.D., Hughes Jones, et al. (1993) Human anti-self antibodies with high specificity from phage display libraries. EMBO. J. 12, 725.

Hawkins, R.E., Russell, S.J. and Winter, G. (1992) Selection of Phage Antibodies by Binding Affinity Mimicking Affinity Maturation. J. Mol. Biol. 226, 889.

Hoogenboom, H.R. and Winter, G. (1992) human antibodies from synthetic repertoires of germline vh gene segments rearranged in vitro. J. Mol. Biol. 227, 381 . 
Huston, J.S., Levinson, D., Mudgett-Hunter, M., Tai, M., Novotny, J., Margolies, M.N., Ridge, R.J.. Bruccoleri, R.E., Haber, E., Crea, R. and Opperman, H. (1988) Protein engineering of antibody binding sites: recovery of specific activity in an anti-digoxin single chain Fv analogue produced in E-coli. Proc. Natl. Acad. Sci. U.S.A. 85, 5879.

Karlsson, R., Fagerstam, L., Nilshans, H. and Persson, B. (1993) Analysis of active antibody concentration. Separation of affinity and concentration parameters. J. Immunol. Methods 166,75 .

Karlsson, R.. Michaelsson, A. and Mattsson, L. (1991) Kinetic analysis of monoclonal antibodyantigen interactions with a new biosensor based analytical system. J. Immunol. Methods 145, 229.

Karlsson, R., Roos, H., Fagerstam, L. and Persson, B. (1994) Kinetic and concentration analysis using BIA technology. Methods Companion Methods Enzymol. 6, 99.

Liedberg, B. and Lundström, I. (1993) Principles of biosensing with an extended coupling matrix and surface plasmon resonance. Sens. Actuat. 11, 63.

Löfăs, S. and Johnsson, B. (1990) A novel hydrogel matrix on gold surfaces in surface plasmon resonance sensors for fast and efficient covalent coupling of ligands. J. Chem. Soc. Chem. Commun. 21, 1526.

Marks, J.D., Hoogenboom, H.R., Bonnert, T.P., McCafferty, J., Griffiths, A.D. and Winter, G. (1991) By-passing immunization. Human antibodies from V-gene libraries displayed on phage. J. Mol. Biol. 222, 581.

McCafferty, J., Griffiths, A.D., Winter, G. and Chiswell, D.J. (1990) Phage antibodies: filamentous phage displaying antibody variable domains. Nature 348, 552.

O'Shannessy, D.J., Brigham Burke, M., Soneson, K.K., Hensley, P. and Brooks, I. (1993) Determination of rate and equilibrium binding constants for macromolecular interactions using surface plasmon resonance: use of nonlinear least squares analysis methods. Anal. Biochem. 212, 457.

\section{Acknowledgements}

The authors thank B. van der Weyer for the kind gift of high purity hCG. 


\section{Chapter 4}

\section{Single-CHAIN FV'S AgAinst HCG FROM A MURINE IMMUNE LIBRARY}

Submitted as

A comparative molecular analysis of hybridoma-derived and combinatorial library-derived monoclonal antibodies to human Chorionic Gonadotropin

Hans J.W. de Haard, Bert Kazemier, Cora de Jongh, Ebo Bos, Bob van Gemen, Jan-Willem Arends, and Hennie R.J.M. Hoogenboom 


\section{Abstract}

In the last decades, detection of urinary human chorionic gonadotropin (hC.G) has been the hall mark of early pregnancy tests. Panels of antibodies, made via hybridoma technology, exist that have solved the intrinsic complexity of this test, which are the presence in pregnancy urine of (i) high concentrations of biologically inactive fragments derived from the $\beta$-unit of hCG, and (ii) the closely related human luteinizing hormone (hLH) and human follicle stimulating hormone (hFSH). Here, we have used phage display to study the murine immune response against hCG and to isolate antibodies to many different epitopes on this antigen. Immune antibody repertoires were constructed from immunised mice and single chain antibodies (scFv's) with hCG binding were retrieved from these repertoires by biopanning or selection using labelled hCG in solution. ScFv"s with most of the expected binding specificities were retrieved: (i) $\mathrm{scFv}$ 's specific for the $\alpha$-subunit, thus reacting with the gonadotropins hCG, hLH and $h F S H$; (ii) scFv's reactive against the $\beta$-subunit of both hCG and $\mathrm{hLH}$; (iii) scFv's specific for the $\beta$ subunit of hCG; and (iv) an antibody, which only binds to the intact heterodimer of hCG and hLH. Analysis of the variable ( $\mathrm{V}_{-}$) gene sequences indicate that the antibodies of the (ii) group use $\mathrm{V}$-germline segments of the same $V_{H}$ family as those of the hybridoma-derived monoclonal antibodies (mABs) with the same specificity, but that in the other groups no other comparable genetic homologies were found. The availability of the encoding Ab-genes in combination with phage display allows the manipulation of affinity and avidity, which might lead to the creation of molecules for a new generation of sensitive and rapid pregnancy assays.

\section{Introduction}

The hormone human chorionic gonadotropin (hCG) regulates steroid secretion by the corpus luteum during the early stages of pregnancy, until the placenta itself can take over this function. hCG is a member of the family of glycoprotein hormones, which also contains the structurally closely related human luteinizing hormone (hLH), follicle stimulating hormone (hFSH) and thyroid stimulating hormone (hTSH). The hormones are heterodimers sharing an identical $\alpha$ chain. and an unique $\beta$-subunit that confers target-specificity. The $\alpha$-chain contains 92 residues with five disulphide bridges, while the $\beta$-chain, with six disulphide bridges. varies in length from 114 residues (in $\mathrm{hI} . \mathrm{H}$ and $\mathrm{hFSH}$ ) to 145 (in hCG). The recently determined structure of hCG revealed a similar conformation for both individual subunits, each having a central cysteine knot with, on one site of the knot, a loop of double stranded $\beta$-sheet-like structure, and, on the other site, two hairpin loops [1]. Despite differences in the $\beta$-chain, it was presumed that the related hormones have a similar hasic structure. because all cysteine residues involved in the knot are 
conserved in all members. With respect to the first 114 amino acid residues of the $\beta$-chain, hLH is more homologous ( $85 \%$ ) to hCG than to hFSH (36\%); this high degree of homology of hLH and hCG reflects their capacity to bind to the same receptor.

Measurement of hCG, intact or nicked, possibly combined with the detection of free $\beta$ subunit or the proteolytical fragments derived from the hCG $\beta$-chain $[2,3]$ (such as the $\beta$-core fragment $(\beta$-cf)), forms the basis of immunoassays for diagnosis of pregnancy (excellently reviewed in [4]). From the structural and sequence similarity, it will be clear that the presence of high concentrations of $\mathrm{hLH}$, also found in pregnancy urine, will complicate such assay [5]. Another problem is the presence of the major break-down product of $\mathrm{hCG}$, the urinary $\beta$-core fragment, which contains two polypeptide chains hCG $\beta$ 6-40 disulphide bridged to hCG $\beta$ 55-92; it can be found in pregnancy urine at even higher concentrations than intact hCG, but also in patients with different types of cancer $[6,7]$. Fortunately, the application of certain combinations of monoclonal antibodies in a sandwich-format enables the specific detection of the intact heterodimeric hCG, which is the bioactive molecular form. Recently assays have been developed for the detection of degraded or dissociated hCG, aiming at the diagnosis of trophoblast disease, Down syndrome pregnancies, preeclampsia, and testicular and bladder cancers.

During the last decades large panels of murine monoclonal antibodies were generated for diagnostic assays, and much knowledge has been gathered on the recognised epitopes of hCG. With hybridoma technology it proved to be relatively difficult to get antibodies to hCG specific epitopes, since most are hLH cross-reactive (see for instance [5]) or recognise one of the free subunits. In this study we used phage display to obtain a series of anti-hCG antibodies. Until now, only a few reports have been dedicated to the molecular comparison between phage displayed and hybridoma derived antibodies [8], therefore combining the knowledge of sequences and specificities of the mABs with the data from the phage antibodies resulted in a detailed analysis of the murine immune response against hCG. In addition, we evaluated combinatorial library technology for the isolation of antibodies, with good characteristics (in terms of specificity and affinity) for pregnancy testing.

\section{Materials and methods}

\subsection{Sequence analysis of $\alpha h C G m A B$ 's}

In this study four $\alpha$-hCG murine monoclonal antibodies (IgGl, kappa), made by immunisation of Balb/c-mice ( $100 \mu \mathrm{g}$ hormone, purified from urine [9], was used for the primary injection, $25 \mu \mathrm{g}$ as boost) were examined. One group of antibodies, containing $\mathrm{mAB}$ 147, $\mathrm{mAB} 212$ and $\mathrm{mAB} 214$, recognise the $\beta$-subunit, while $\mathrm{mAB} 121$ binds the holo-form of the hormone. RNA was isolated [10] from $5 \times 10^{7}$ hybridoma cells yielding approx. $500 \mu \mathrm{g}$ RNA. For the determination of the complete wild-type regions, the $\mathrm{V}$-genes were amplified on random 
primed cDNA, synthesised from $0.5 \mu \mathrm{g}$ total RNA, with primers located in the constant region and the leader sequence (listed in [11]). The PCR-fragments were cloned into the T-vector (Promega, Madison, USA) and completely sequenced.

\subsection{Library construction and selections}

Two Balb/c mice were immunised with $100 \mu \mathrm{g}$ hCG (same source as mentioned before) with Freunds complete adjuvant. After ten days, one mouse was sacrificed and its spleen collected. The other mouse was boosted one month after the first immunisation with $25 \mu \mathrm{g} \mathrm{hCG}$ in combination with incomplete adjuvant. Fourteen days later the spleen was also collected from this mouse. Total RNA was extracted from the spleens [10] (360 $\mu \mathrm{g}$ for the boosted mouse and $92 \mu \mathrm{g}$ for the other one); $10 \mu \mathrm{g}$ was transcribed into cDNA using random priming.

The amplification of $\mathrm{V}$-genes (including the sequence of the used oligonucleotide primers), and their cloning was performed as described [12]; the IgM specific primer contains the sequence 5'-AGA CAG GGG GCT CTC GCA GGA GAC-3'. For library construction we used the phagemid vector pV1, which is identical to pHEN1 [13], except that it has the FLAG-tag [14] in stead of the $c-m y c$ derived tag. Transformations were performed by electroporation [15] in JM101. The ratio of insert containing clones was determined with PCR analysis as was performed by Marks and colleagues [16].

The rescue of phagemid particles was essentially performed as described [16]. For biopanning, hCG purified from urine [9], was coated in a six wells tissue culture plate (Costar, Cambridge MA., USA) at a concentration of $25 \mu \mathrm{g} / \mathrm{ml}$ in $50 \mathrm{mM} \mathrm{Na}_{2} \mathrm{CO}_{3} \mathrm{pH} 9.6$. Selections were carried out using $10^{11}$ phage particles in $1 \mathrm{ml}$ of sample diluent (PBS; $20 \%(\mathrm{v} / \mathrm{v}$ ) normal goat serum; $1.1 \%(\mathrm{v} / \mathrm{v})$ triton $\mathrm{X}-100)$. The wells were washed and bound phage eluted with $0.1 \mathrm{M}$ triethylamine (TEA) as described [16]. For selection in solution, hCG was biotinylated with NHS-Biotin (Pierce, Rockford, USA) at a ratio of twenty molecules Biotin to one molecule of hCG. Phage, $10^{11}$ particles in $1 \mathrm{ml}$ sample diluent, and $100 \mathrm{nM}$ biotinylated hCG were incubated for two hours; the phage antigen complexes were captured with streptavidin-coated paramagnetic beads (Promega, Madison, USA) [17]. Further washes and the elution with TEA was performed as described [16].

\subsection{Screening of individual clones and purification of $s c F v$}

Individual clones were screened for the production of hCG-reactive scFv's as described before [16]. Culture supernatants were diluted five-fold in sample diluent and tested in ELISA on plates coated with $4 \mu \mathrm{g} / \mathrm{ml} \mathrm{hCG}$ in $50 \mathrm{mM} \mathrm{Na} \mathrm{CO}_{3} \mathrm{pH}$ 9.6. ScFv bound to hCG was detected with mouse anti-FLAG antibody M2 (Kodak IBI, New Haven, USA) and anti-mouse-HRP conjugate (DAKO, Glostrup. Denmark). Unique clones, identified by Bst NI-fingerprinting, were sequenced with a thermocycling kit (Amersham, Buckinghamshire, UK) on the ALF-sequencing machine (Pharmacia. Lund, Sweden). The antibody families used in scFv-genes were determined 
by the Family Assignment Software available on the Kabat Database homepage (http://immuno.bme.nwu.edu/famgroup.html). The most homologous germline $\mathrm{V}_{\mathrm{H}^{-}}$and $\mathrm{V}_{\mathrm{K}^{-}}$ sequences were found in the "Germline gene directories of the mouse" homepage (http://www.ibt.unam.mx/ almagro/V_mice.html).

$\mathrm{ScFv}$ was affinity purified on hCG-columns, prepared by coupling of $8.4 \mathrm{mg}$ hCG per gram Tresyl sepharose according to the suppliers instructions (Pierce, Rockford, USA), after refolding of the total bacterial proteins from a $50 \mathrm{ml}$ culture, as was described elsewhere [18]. The yield was determined by measuring the optical density at $280 \mathrm{~nm}$, assuming that a scFv has a molar extinction coefficient $\left(E^{1 \%}{ }_{280 \mathrm{~nm}}\right)$ of 14.3 [19]. The purity was evaluated on a Coomassie stained gel.

\subsection{Analysis of specificity with ELISA, Western blot, surface plasmon resonance and pepscan}

The hCG-preparation purified from urine, and immuno-affinity purified recombinant hLH, hFSH and hFSH-CTP, all produced in CHO-cells $[20,21]$, were used for Westem blot studies as was described [22] and for ELISA. All selected scFv's were tested in ELISA using a tenfold diluted periplasmic fraction on hCG, $\beta$-cf (purified from urine according to previously described methods [23]), and hFSH (all coated at $4 \mu \mathrm{g} / \mathrm{ml}$ in $\mathrm{Na}_{2} \mathrm{CO}_{3} \mathrm{pH} 9.6$ ) using the method described in section 3.3. For Western blot analysis, between 0.5 and $1 \mu \mathrm{g}$ of each hormone was loaded per lane; the proteins were diluted in sample buffer with and without DTT, and boiled during 5 minutes or directly loaded on gel without heat-treatment. The blots were incubated during 16 hours at room temperature with 1 to $6 \mu \mathrm{g} / \mathrm{ml}$ purified scFv or with $3 \mu \mathrm{g} / \mathrm{ml}$ monoclonal antibody, diluted in TBST (10 mM Tris-HCl; $150 \mathrm{mM} \mathrm{NaCl} ; 0.05 \%$ (v/v) Tween 20; $\mathrm{pH} \mathrm{8.0).} \mathrm{ScFv} \mathrm{was}$ detected with mouse anti-FLAG M2 and anti-mouse Ig alkaline phosphatase (AP)-conjugate (Promega. Madison, USA), and the monoclonal antibodies with anti-mouse AP-conjugate.

The specificity of the ScFv's was further characterised with surface plasmon resonance on a BIAcore machine (BIAcore AB, Uppsala, Sweden) using the different glycoprotein hormones. The recombinant hLH, hFSH and hFSH-CTP were biotinylated with NHS-Biotin (Pierce, Rockford, USA) as was described before for hCG. The biotinylated hormones were immobilised on streptavidin coated chips (SA-chip, BIAcore AB, Uppsala, Sweden), yielding a surface with 1375 RU hCG, 1906 RU hLH, 1529 RU hFSH, and with 1221 RU hFSH-CTP. Purified scFv's were injected on all surfaces at concentrations varying from 12 to $99 \mu \mathrm{g} / \mathrm{ml}$, while the mAB's were used at a concentration of $50 \mathrm{ng} / \mathrm{ml}$.

Epitope mapping was performed with the pepscan method [24]. Overlapping dodecapeptides covering the entire sequence of both the $\alpha$ - and $\beta$-chain, coupled to the solid support, were tested with scFv. The purified scFv of clone A6l was diluted to a concentration of $3 \mu \mathrm{g} / \mathrm{ml}$ in PBS containing $5 \%(v / v)$ horse serum, 5\% (w/v) BSA and $1 \%(v / v)$ Tween 20. After 16 hours incubation at $4^{\circ} \mathrm{C}$ scFv bound to the peptides was detected with anti-FLAG M2 and anti-mouse Ig horseradish peroxidase (anti-mouse HRP) conjugate. 


\subsection{Affinity measurements in solution}

As was recommended in the BLAevaluation software for the measurement of the "in solution" alfinities, we prepared a high density coated CM-chip with hCG (4200 RUs) using the NHS/EDC-kil (Pharmacia, Lund, Sweden). A fixed amount scFv, Fab or mAB, which gave a signal between 1000 and 1500 RUs when injected on the hCG chip. was mixed with a variable amount of antigen in a volume of $60 \mu \mathrm{l}$ with Hepes Buffered Saline (HBS; $10 \mathrm{mM}$ Hepes. 3.4 mM EDTA, $150 \mathrm{mM} \mathrm{NaCl}, 0.05 \%$ (v/v) surfactant P20, $\mathrm{pH} 7.4$ ) as dilution buffer. After an one hour period, needed for settlement of equilibrium, the samples were injected. For the quantification of free $A B$ a standard curve was prepared by injection of a dilution series of the tested $\mathrm{AB}$ on the same flow cell as was described before [25].

\section{Results}

\subsection{The murine immune response against hCG as found by hybridoma technology}

During the last twenty years, large numbers of anti-hCG mABs have been made for diagnostics. In pregnancy assays it is of importance to detect the intact heterodimer, and not one of the free fragments or closely related hormones. The combination of antibodies with different specificities solves these problems, for instance capture with an $\alpha$-subunit specific antibody and detection with a $\beta$-unit, hCG-specific antibody ensures the specific detection of the intact hormone of interest, being hCG. In the last decade, extensive screening of hybridomas derived from 32 fusions in our laboratories yielded a number of interesting mABs. The most frequently retrieved type of antibodies are the $\beta$-subunit, hLH cross-reactive antibodies, with mAB 212 and mAB 214 as representatives. Another often found specificity was directed against the $\alpha$-subunit, here represented by $\mathrm{mAB} 116$. Only two $\mathrm{mABs}$, which recognised the intact heterodimer of hCG and hLH, were isolated; we chose mAB 121 from this group for further analysis. It turned out to be extremely difficult to select hCG-specific antibodies: from all fusions a single mAB was obtained, that recognised the carboxy terminal peptide of the $\beta$-unit of hCG ( $\beta$-CTP; nomenclature in [26]); it was coded $\mathrm{mAB} 293$.

For studying the immune response against hCG on a molecular level, we used phage library technology. First of all, we were interested to see whether the specificities of the selected recombinant antibodies would match with the hybridoma derived antibodies, and secondly. if the sequences of both types of antibodies would be related. Therefore the sequences of the V-regions were determined from mABs 121, 147, 212 and 214. Their potential application in diagnostics was evaluated by analysis of specificity and affinity. The next paragraphs will discuss the selection and characterisation of the recombinant antibodies. 


\subsection{Construction of murine librarics and selection of anti-hCG phage antibodies}

For the generation of combinatorial libraries, we immunised two Balb/c mice, the strain originally used for the generation of the hybridomas, with $100 \mu \mathrm{g}$ purified hCG. Different immunisation schemes were applied to investigate the effect of maturation on the affinity and the specificity of the selected antibodies. One mouse (\#1) was sacrificed 10 days after a single immunisation; its spleen was removed and used for isolation of total RNA. The other mouse (\#2) was boosted after one month with $25 \mu \mathrm{g} \mathrm{hCG}$ and sacrificed a fortnight later (this was the standard protocol for the generation of hybridomas). Random primed cDNA was synthesised and applied as template for the amplification of $\mathrm{IgG}$ or IgM specific variable heavy chain fragments $\left(V_{H}\right)$ for mouse \#1, and IgG specific $V_{H^{-}}$-regions only for mouse \#2, and kappa light chain region $\left(V_{K}\right)$ fragments from both mice. The oligonucleotide primers used for amplification were designed on the basis of the encoding amino acid sequences of published mouse antibodies. The $\mathrm{V}_{\mathrm{H}^{-}}$and $\mathrm{V}_{\mathrm{K}^{-}}$encoding DNA-segments were coupled by splicing by overlap extension, as was described before [8], and cloned in phagemid pV1. Two libraries were made for mouse \#1, encoded $\mathrm{Ml}$ containing the IgM derived $\mathrm{V}_{\mathrm{H}}$-regions with $5 \times 10^{6}$ clones (with $70 \%$ of the clones containing a scFv encoding insert), and library $\mathrm{Gl}$ from the $\mathrm{IgG}$ derived $\mathrm{V}_{\mathrm{H}}$ fragments with $1.4 \times 10^{7}$ cloness (the fraction of clones, which have an insert, was $85 \%$ ). From mouse $\# 2$ only an IgG-derived library was generated, encoded G2, contuining $1.1 \times 10^{7}$ clones $(85 \%$ of the clones had insert).

The rescued phage of library Gl and Ml were selected separately on hCG coated onto a polystyrene surface [16]. After three rounds of panning a 200 -fold enrichment was found in the number of eluted phage relative to the numbers sluted during round 1 . The number of scFv expressing clones increased from 50\% after round 1 to more than $95 \%$ after round 3 , as was judged on morphology of the clones [12]. Among the antigen reactive clones of library Gl, six different fingerprints were identified. Representative clones were taken from each pattern, which were coded A11, A61, A64, A124, C11 and C35. From the IgM library M1 we retrieved two different hCG reactive clones: A6 and A7. The mode of antigen presentation during selection was examined by repeating the selection on library Gl, but instead of immobilised antigen we now used soluble biotinylated hCG (100 nM at each round). The phage titre upon elution increased in round 4 only, with an unexpected 100 -fold decrease in round 2. Three hCG binding clones were identified by fingerprint analysis, i.e. clones A1, A5 and A9. Finally, the $\lg$ G based library G2, made from the spleen of mouse \#2, was used for selection with soluble antigen, yielding a similar enrichment as was seen with library Gl and M1. Four antigen specific clones, coded B3, B4, B6 and B7, were identified.

In the following paragraphs we will discuss the characterisation of our panel of in total 15 different anti-hCG single chain antibodies. The specificity of the selected antibody fragments was determined with Western blot, surface plasmon resonance, and in some cases with pepscan. 
revealing four classes of binding specificities. To complete the comparison with mABs, the affinities were determined with refolded, affinity purified scFvs. Since the applied refolding procedure only yields a fraction of functionally active antibody fragments, the inactive molecules were removed by affinity chromatography on hCG columns. By doing this, overestimation of the protein concentration was avoided by the presence of inactive scFvs, which would lead to lower affinities. Instead of measuring the kinetics of binding. we used the "in solution" method for determination of the affinity [27], which permits the direct comparison between scFvs, intact $\mathrm{mABs}$ and Fabs. The principle relies on the measurement of the amount of free analyte $(\mathrm{AB}$ or $\mathrm{Ag})$ in a pre-established equilibrium mixture. We determined the amount of free antibody in the BIAcore machine according to the method described by Kazemier and colleagues [25]. By plotting the concentration of free $A B$ or the fraction $A B_{\text {free }} / A B_{0}$, in which $\mathrm{AB}_{0}$ is the concentration $\mathrm{AB}$ when no $\mathrm{Ag}$ has been added, against the total concentration $\mathrm{Ag}$, the $K_{d}$ can be deduced.

\subsection{Anti- $\alpha$-chain scFv's clones A6, A7, B6 and B7}

Westem blot analysis, using the recombinant antibody as the detection probe, revealed that the specificity of clone B6 scFv is directed against the $\alpha$-chain of hCG, hLH, hFSH and hFSHCTP; the latter chimera contains the carboxyterminal peptide of the $\beta$-chain of hCG fused to the $\beta$-chain of FSH as described [28]. Although clone B6 was selected with biotinylated hCG from library G2, it recognises all hormone derivatives in both native and denatured form (Fig. 1B). When the gonadotropins were analysed without heat treatment, the intact heterodimer was detected by B6 scFv (marked with $\alpha / \beta$ in Fig. 1B). This specificity pattern is identical to that of the hybridoma produced monoclonal antibody 116 (coded mAB 6 in Fig. 1C).

BIAcore analysis with the different gonadotropins immobilised on sensorchips confirmed the anti- $\alpha$-chain specificity of the scFv observed with Western blot analysis (Fig. 2B). A good response was seen against hFSH (also in ELISA), and against hLH in the sensorgrams (270 RU and 150 RU respectively immediately after injection at $t=260 \mathrm{sec}$ in Fig. 2B). On hCG a much lower signal (approx. 50 RU) was obtained in spite of a similar antigen density on the chip; this is probably caused by the presence of free $\beta$ subunit in the urinary hCG preparation used for immobilisation. Similar responses were obtained with the $\alpha$ subunit specific mAB 116 (420 RU for $h F S H$ and $240 \mathrm{RU}$ for hLH at $t=260 \mathrm{sec}$ in Fig. 2C), including the relative low response on hCG (30 RU). Clones B7, A6 and A7 gave identical sensorgrams as clone B6 (data not shown), suggesting that these also recognised the $\alpha$-chain. In Fig. 4 the affinity curves derived from several anti-hCG mABs and scFvs are shown; Table 2 gives the corresponding $K_{d}$-values. The affinity of the antibody fragment expressed by clone B6 was $35 \mathrm{nM}$ (Table 2), which is slightly lower than of the anti- $\beta$-CTP specific $\mathrm{mAB} 293$ or $\mathrm{mAB} / \mathrm{scFv} 212$ and somewhat better than of $\mathrm{mAB} / \mathrm{scFv} 214$; the two last $\mathrm{mABs}$ are reactive against the $\beta$-subunit. 


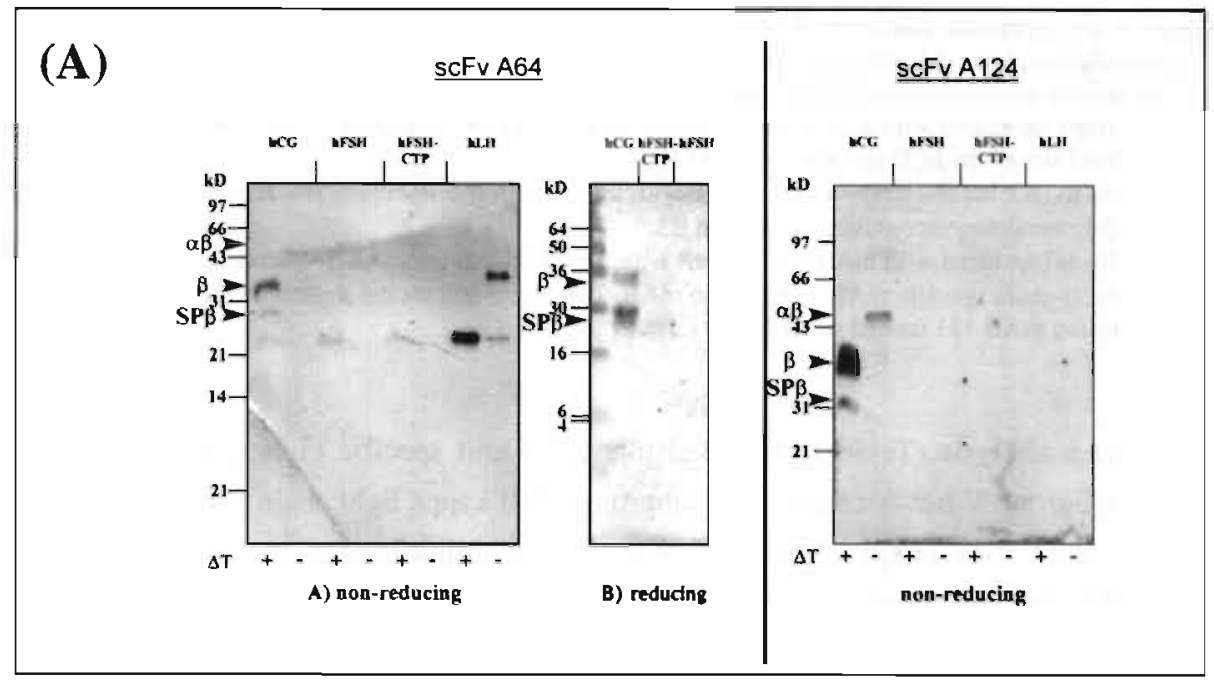

(B)

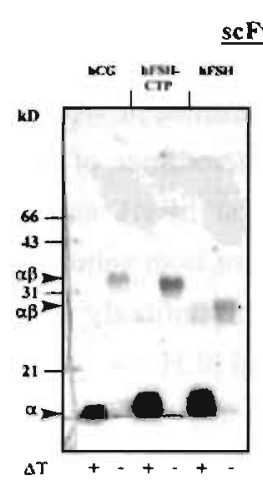

A) non-reducing

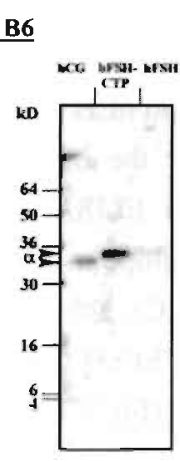

B) reducing

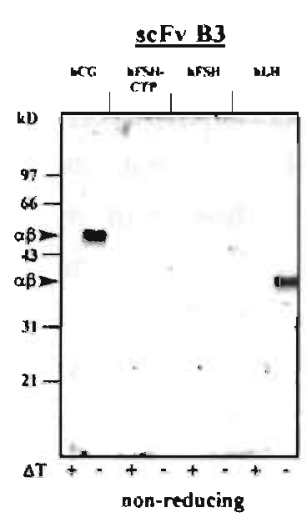

(C)
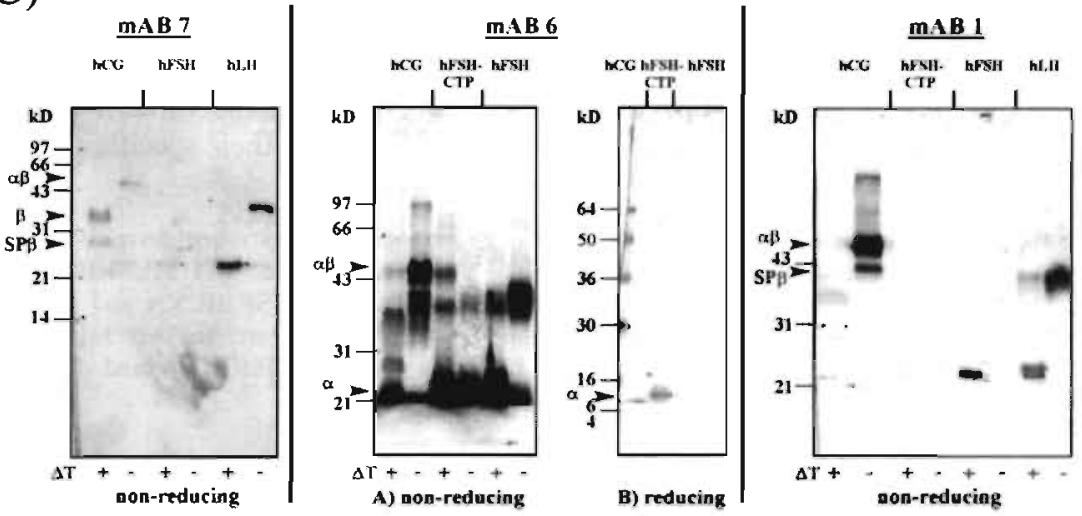
Figure 1 (on previous page). Analysis of the specificities of the scFvs and mABs on Western blot. The gonadotropins were dissolved in non-reducing sample buffer, and boiled ( $\mathrm{T}+$ ) or not heat-treated (T-). ScFvs A64 and B6 also recognised protein, which was denatured with DDT (blots in the middle).

Panel $A$. Blots incubated with anti- $\beta$-subunit scFvs, of the hLH cross-reactive clone $A 64$ on the left, and on the right hand site of the hCG specific clone A124.

Panei $B$. As in (A), but the left blot was probed with the $\alpha$-chain specific clone B6, and the one on the right with the $\alpha / \beta$-heterodimer recognising scFv from $B 3$.

Panel $C$. Blots incubated with mABs; on the left with the anti- $\beta$-subunit $\mathrm{mAB} 147$ (coded mAB 7), in the middle with the $\alpha$-chain specific mAB 116 (coded $\mathrm{mAB} 6$ ), and the one on the right with the anti- $\alpha / \beta$-heterodimer recognising $\mathrm{MAB} 121$ (coded $\mathrm{mAB} 1$ ).

Sequence analysis (Table 1) revealed that all $\alpha$-unit specific clones A6, A7, B6 and B7 contain a subgroup $V$ heavy chain and a subgroup XVII kappa light chain variable region, except clone A7, which uses a kappa family IX. A major variability is seen in the length of the heavy chain CDR3. The closest matches with germline sequences were found with the heavy chain $\mathrm{V}$ region C57G26 and the light chain V-region K2 [29]).

\subsection{Anti- $\alpha / \beta$-chain scFv: clone B3}

The $\mathrm{scF} v$ derived from clone $\mathrm{B} 3$, selected from the library $\mathrm{G} 2$ with biotinylated hCG, recognises the intact heterodimers of hCG and hLH as determined in Western blot (Fig. IB). The individual subunits were not detected, nor the intact heterodimer of hFSH and hFSH-CTP, confirmed by the absence of rexponse in ELISA against hFSH and $\beta$-cf. Apparently the recognised sequence is a conformational epitope made up of both subunits. A similar reactivity was found with $\mathrm{mAB} 12 \mathrm{I}$ ( $\mathrm{mAB} I$ in Fig. $1 \mathrm{C}$ ), although this antibody was shown to have some cross-reactivity with the $\alpha$-chain products of hFSH-CTP and hLH.

The results were confirmed in BlAcore (Fig. 2B), although the responses on hCG and hLH were rather low (40 and $80 \mathrm{RU}$ respectively) for the scFv from clone B3. However, signals are comparable to the signals obtained from mAB 121 (200 and $400 \mathrm{RU}$ on hCG and hLH respectively), taking into account the five-fold difference in molecular weight between an intact IgG and a scFv (see Fig. 2). As was already observed with Western blot, mAB 121 gave some degree of reactivity ( $50 \mathrm{RU}$ ) on $\mathrm{hFSH}$, whereas the scFv did not have the cross-reactivity.

Table 1 (on next page). Alignment of the amino acid sequences of the variable heavy $(A)$ and light (B) chain regions of the anti-hCG scFv-s, grouped upon their specificities, with their germline analogues.

a) On the bottom line of each group of library derived antibodies, the germline derivative is shown. The Genbank-codes and accession number of the germline $\mathrm{V}_{\mathrm{H}}$-regions are: for V11 MUSIGHVP and J00525 respectively, for C57G26 MUSC57G26V and L33937. for GLvh50 MUSIGHCVA and L17134. and for 37A11 MUSIGHVAD and M18951. For the germline $V_{L}$-regions these are: for Ars MUSIGKCMN and M15520 respectively, for 24B MUSIGKVV and K02418, for K2 MUSIGKVA and J00545. for $21 \mathrm{~A}$ MMVK21A and X16954, and finally for 21C MUSIGKVR3 and K02161:

b) Library, from which the clone was selected:

b) Numbering of the residues and classification of the heavy and light chains was performed according to Kabat et al. (1991).Differences from the first member of the group are indicated with uppercase characters, primer encoded differences with lowercase characters:

a) The specificity of the scFv's are indicated with a ( $(\alpha-$-unit) or b ( $\beta$-unit);

a) The number of somatically mutated germline residues are also shown (\#mut), the mutated residues themselves are indicated with boxes. 


\section{A. Heary chain}

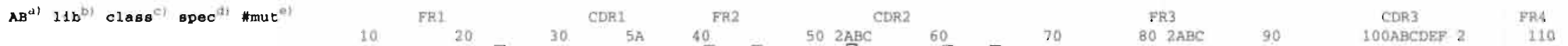

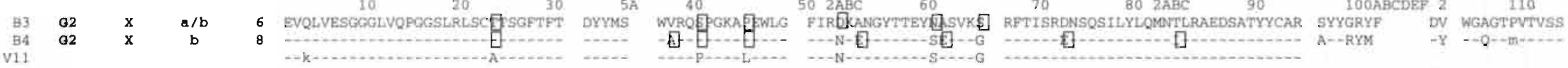

$$
\begin{aligned}
& \begin{array}{llll}
B 6 & \text { G2 } & \text { V } & \text { a } \\
\text { B7 } & \text { G2 } & \text { V } & \text { a } \\
A 6 & \text { M1 } & \text { V } & \text { a } \\
\text { A7 } & \text { M1 } & \text { V } & \text { a }
\end{array}
\end{aligned}
$$

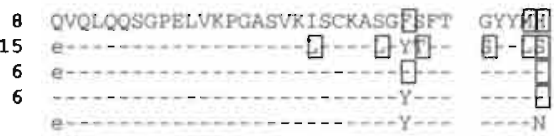

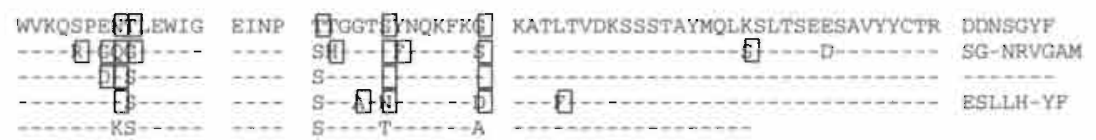

\begin{tabular}{|c|c|c|c|c|c|c|}
\hline A64 & a1 & VII & b & 3 & EVQVQQSGAELMKPGASVKISCKATGYTFS & SYWIE \\
\hline A61 & Q1 & VII & b & 4 & $q \cdots$ & \\
\hline A11 & GI & VII & b & 6 & $\cdots 1 \cdots p \cdots$ & \\
\hline A1 & G1 & VII & b & 10 & -.. & \\
\hline AS & G1 & VII & b & 14 & $q-\cdots+1 \ldots b-$ & 证 \\
\hline $\begin{array}{l}\text { C11 } \\
37 A 11\end{array}$ & G1 & VII & b & 4 & $q-11+p-1, \ldots$ & \\
\hline
\end{tabular}
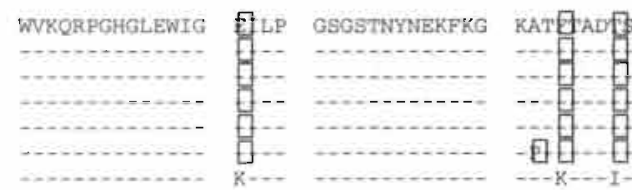

\section{B. Light chain}

\section{AB}

B3

Ars

\begin{tabular}{|c|c|}
\hline $11 b^{b !}$ & clangel \\
\hline G2 & XIII \\
\hline$G 2$ & VII \\
\hline G2 & XVII \\
\hline G2 & XVII \\
\hline M1 & XVII \\
\hline G1 & $I X$ \\
\hline GI & IX \\
\hline 31 & IX \\
\hline G1 & IX \\
\hline 91 & IX \\
\hline G1 & IX \\
\hline 01 & IX \\
\hline M1 & IX \\
\hline
\end{tabular}

B4

248

B6 $7 A B C D E 30$

FR2

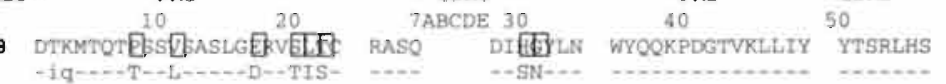
60 FR3. 80

$\mathrm{CDR} 3$ $90 \quad 5 A \quad 100$

FRA -

2 DIVMTONAFSNPVTLGTSASISC RSSKSLLHSNGITYLE WYLOKPGQSPQLLIY QMSWLAS GVPDRFSSSGSGTDETLRISRVEAEDVGVYYC Q.ULLLP T PGAGTKLELKR

4 DIKMTOSPASLSASVGETVTITC FASG

MIHAY

NAKTLAD

[6- [e

QHEYTP WT PGCGIKLEIKR

$12-\cdots+\cdots+$ 国

10 ENVLTQSPASLAVSLGQRATTSC

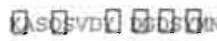

Rs

GVPARFSGSGSGTDFSLNIHPMEEDPTAMYCC.

\# - -

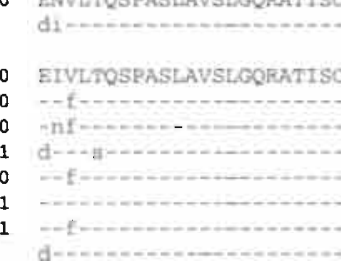

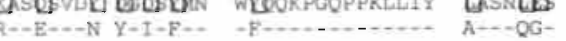

RASESVDS YGNSFMH WYOKPGOPPKLLIY RASNLES

GIPARFSGSGRTDFTLTINPVEADDVATYYC QQSREDP WT POCRTYLBLKR

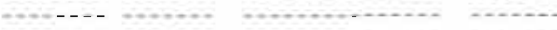

(n)
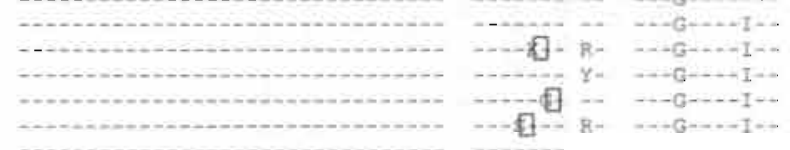
The affinity of the antibody fragment produced by clone B3 (Fig. 4A), is lower than found for the mABs or scFv B6: $80 \mathrm{nM}$ (Table 2). A comparison with mAB 121 could not be made, since its affinity was not determined.

Alignment of the heavy chain sequence of clone B3 revealed the use of a subgroup $X$ variable region, derived from the germline sequence coded V11 [30] (Table 1). The scFv of clone B4, reactive against the $\beta$-subunit of hCG and hLH (discussed below), contains a $V_{H}$ homologous to the one of clone B3, but its CDR3 is different in length and sequence, while the light chain variable regions of both clones are completely different (clone B4 uses a class VII kappa light chain derived from the germline 24B [31]. Clone B3 has a kappa variable light chain variable region of class XIII, derived from the germline analogue Ars [32].

\section{5 hLH cross-reactive antibody fragments recognising the $\beta$-subunit of $h C G$}

The majority of clones selected from library G1, either via panning (clones A61, A64, Al1, and $\mathrm{C} 11$ ) or with biotinylated antigen (clones $\mathrm{Al}$ and $\mathrm{A5}$ ), were reactive with the $\beta$-subunit of urinary hCG. In ELISA the fragments gave positive responses with hCG and its degradation product $\beta$-cf. Clone A64 was chosen for detection of the hormones on Western blot: a clear reactivity was obtained against the $\beta$-unit (MW $36 \mathrm{kDa}$ ) of hCG and hLH, in non-denatured and denatured form (Fig. 1A). In the hCG-preparation purified from urine a product derived from the B-subunit (MW $28 \mathrm{kDa}$ ) was detected by the scFv's in non-denatured but also in denatured form, suggesting that it can not be $\beta$-core $(\beta$-cf) or the carboxyterminal part of nicked hCG (residue 48 to 145 ; for discussion of fragments see $[4,33,34]$ ). When the hCG preparation was analysed on gcl, non-reduced and without heat treatment, the low molecular product could not be detected by $\beta$-unit recognising antibodies. suggesting that it is not a free fragment. Apart from free $\beta$ subunit, the antibodies recognised a molecule on blot with a somewhat lower molecular weight $(40-42 \mathrm{kDa})$ as the intact heterodimer $(47 \mathrm{kDa})$. We therefore believe, that the molecule represents the complete $\beta$-unit with an altered glycosylation pattem, previously described as the $\beta$-unit of the small form of hCG (SP-hCG $\beta$ ) [35], with shorter carbohydrate chains, rather than one of the known degradation products.

Figure 2 (on next page). Binding analysis of the scFv's directed against hCG using surface plasmon resonance (BlAcore).

A. Analysis of the anti- $\beta$-subunit recognising recombinant antibodies. On the left the overlay plot of the hLH cross-reactive seFv from clone $\mathrm{AS}$ on $\mathrm{hCG}$, hFSH, and hLH is shown. From $t=140 \mathrm{sec}$ to $t=260 \mathrm{sec}$ the purified fragment was injected, subsequently the surface was washed with Hepes-buffered saline (HBS) buffer. On the left panel the overlay plot of the hCG specific scFv produced by clone A124 is shown.

B. Sensorgrams of the anti- $\alpha$-chain recognising scFv from clone B6 (left), and of the anti- $\alpha / \beta$-heterodimer scFv from clone B3 (right).

C. For comparison sensorgrams were made from the anti- $\beta$-chain mAB 147 (lett). the anti- $\alpha$-chain reactive $\mathrm{mAB} 116$ (right), and the anti- $\alpha / \beta \mathrm{mAB} 121$ (below). 
(A) anti- $\beta$-core
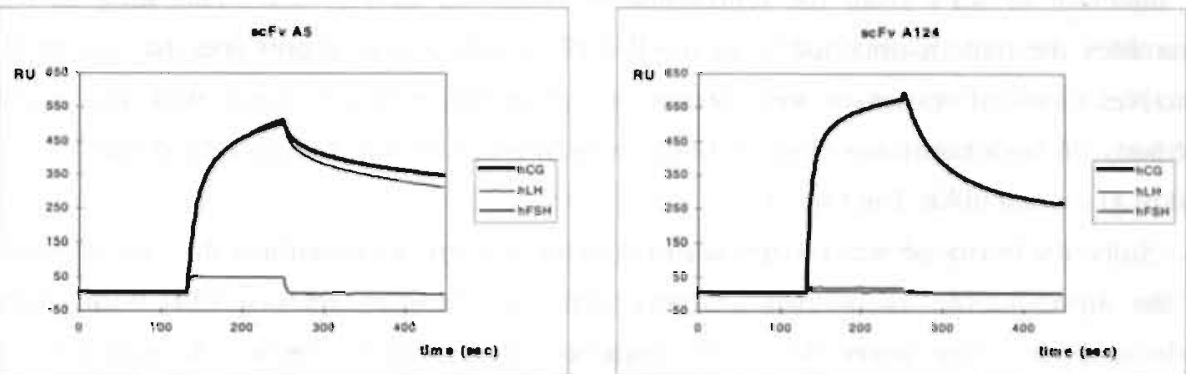

(B) anti- $\alpha$

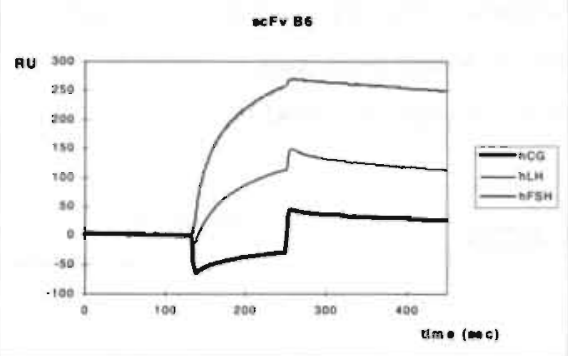

anti- $\alpha / \beta$

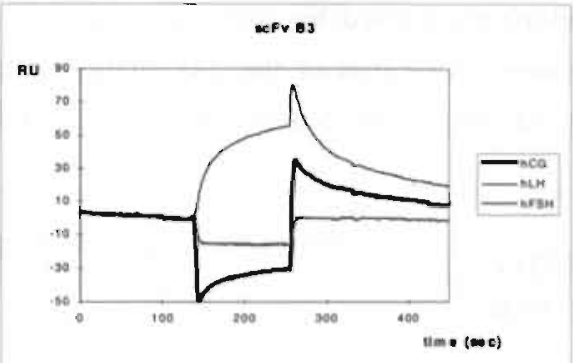

(C) anti- $\beta$-core

anti- $\alpha$
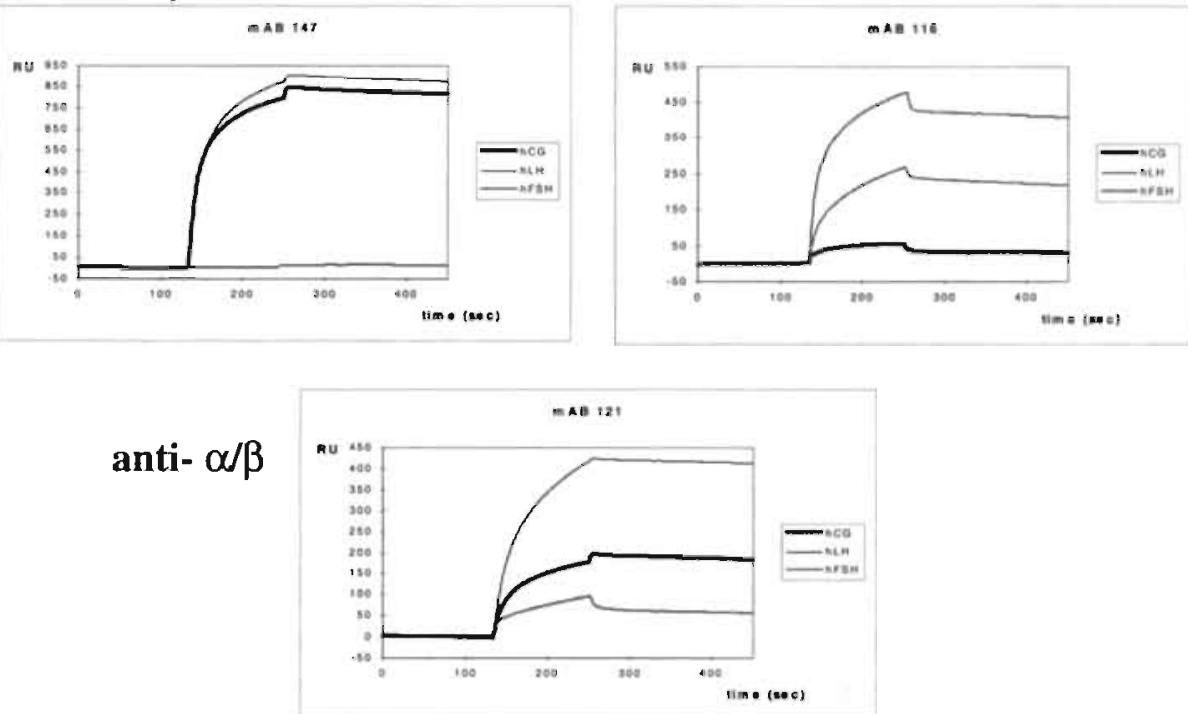
In BIAcore a nearly identical response was found with hCG and hLH (see signals obtained by injection of scFv from the representative clone A5 in Fig. 2A). This kind of reactivity resembles the pattern obtained from $m A B$ 147, which is also $\beta$-unit specific and hLH crossreactive: identical responses were found in surface plasmon resonance with this monoclonal antibody on both hormones (Fig. 2C), while on Western blot it detected the $\beta$-unit of both hCG and $h L H$ (coded $\mathrm{mAB} 7$ in Fig. 1C).

Since the hormone was recognised in denatured form, we examined the epitope recognition of the representative clone A61 in more detail by pepscan analysis [24] with overlapping dodecapeptides, that cover the entire sequence of both hCG chains. A main peak can be identilied in the aminoterminal part of the $\beta$-unit (Fig. 3). The sequence of the first aminoterminal peptide is SKEPLRPRCRPI, and the carboxyterminal peptide of the series of eight reacting peptides contains the sequence RCRPINATLAVE, thereby indicating that the epitupe recognised by $\mathrm{A} 6 \mathrm{I}-\mathrm{scFv}$ includes the sequence RCRPI, which is also present in the aminoterminal part of the $\beta$-core fragment. Another lower peak can be discriminated around peptide number 65 , but the irregular pattern suggests non-specific binding.

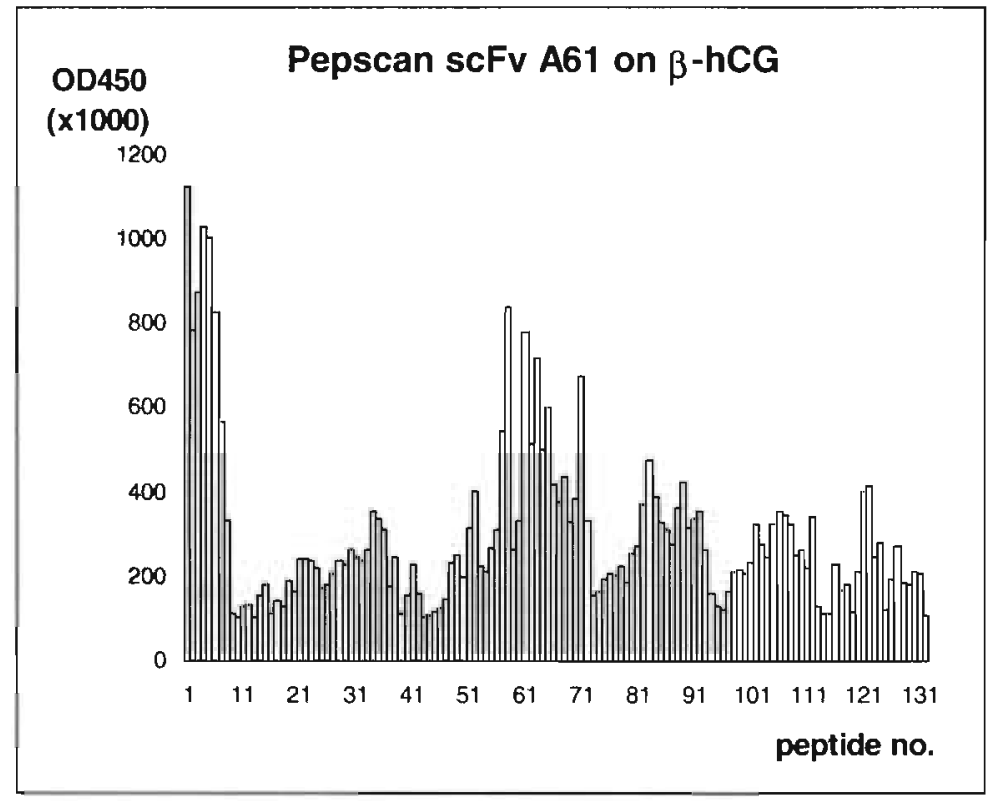

Figure 3. Pepscan results using the $\beta$-chain specific scFv expressed by clone $A 61$. The analysis was performed with the overlapping dodecapeptides derived from the $\beta$-subunit of hCG, starting with the peptide number 1 with the sequence SKEPLRPRCRPI and ending with peptide 134 with the sequence LPGPSDTPILPQ.

The affinities from a limited number of antibody fragments were measured. The A64-scFv has an affinity of $20 \mathrm{nM}$ (Fig. 4A) and the very homologous A61 $35 \mathrm{nM}$ (Table 2; for discussion of sequences see next paragraph). ScFv Al has an approximately ten-fold lower affinity (300 nM; see Fig. 4A and Table 2), but coinciding with this difference in affinity some deviations in 
sequence can be observed within FR1 of $V_{H}$ and CDR3 of $V_{L}$ when compared with clone A64 (discussed in next paragraph).

All anti- $\beta$-unit antibodies have a subgroup VII heavy chain variable region, with some variability in FR1- and CDR1-region (Table 1). Up to 8 differences (not primer encoded) in 30 residues for FR1 and 3 differences in 5 residues for CDR1 were found for clone A5, and 6 in FRl and 1 in CDRl for the previously mentioned $A 1$ in comparison with the $V_{H}$ germline segment encoded 37A11 [36]. The only type of kappa light chain variable region found in these antibodies was of subgroup IX, derived from the germline analogue $21 \mathrm{C}$ [37]. Considering the sequence of $V_{x}, A l$ was again the most divergent clone with two differences in CDR3.

One of the three clones from library $\mathrm{G}_{2}$. clone B4-scFv, recognised also the $\beta$-chain of $\mathrm{hC}(\mathrm{i}$ and hLH. In ELISA an indeterminate signal was found with $\beta$-cf. Its reactivity pattern, as observed on Western blot and in BIAcore (data not shown), was identical to what was found with the clones derived from library G1. Its heavy and light chain sequence completely differs from the Gl derived, $\beta$-subunit recognising antibodies (Table 1), suggesting that the epitopes involved might be different.

\subsection{Reactivity against $\beta$-unit and $h C G$-specific: clones A9, A124 and C.35}

Phage antibodies specific for the $\beta$-chain of hCG were selected from library $\mathrm{G} 1$ using both selection methods (clones A9, A124 and C35). In ELISA positive signals were obtained with $\mathrm{hCG}$ and $\beta-\mathrm{cf}$. but not with hFSH. On Western blot no cross reaction with hLH was seen, as is shown for the representative clone A124 (Fig. 1A). As a control the hLH cross-reactive scFv A5 was used; this clone gave stronger signals against hLH than against hCG on blot, while the hLHreactivity was completely missing for A124 (compare the blot incubated with hLH cross reactive scFv A64 on the left hand with the blot on the right incubated with hCG specific scFv A124 in Fig. 1A). Similar patterns were obtained for the other clones.

This unique hCG-specificity was confirmed by surface plasmon resonance analysis. Injection of the purified scFv's derived from these clones on the hCG surface resulted in high responses. which were comparable to those found for the hLH-cross reactive antibodies, but on hLH, hFSH, and hFSH-CTP no binding could be detected (see Fig. 2A: the left sensorgram shows the binding of $550 \mathrm{RU} \mathrm{scFv}$ at $\mathrm{t}=260 \mathrm{sec}$ on the hCG- and hLH-surface for the crossreactive clone $\mathrm{A} 5$, while an identical amount of scFv A124 was captured only by hCG, as is shown in the sensorgram of the right panel).

Within the group of the hCG-specific scFv's, a big difference in affinity was found; the affinity of ScFv A9 was rather low $(330 \mathrm{nM})$, while the other two antibody fragments had a tenfold higher affinity ( $30 \mathrm{nM}$ and $45 \mathrm{nM}$ for $\mathrm{C} 35$ and $\mathrm{A} 124$ respectively), comparable to the values found for the $\mathrm{hLH}$ cross-reactive or the $\alpha$-chain specific scFv's.

The hCG specific clones used a subgroup $\mathrm{V}$ heavy chain, derived from the germline sequence coded GLvh50 [38] (Table 1). Although all clones contained a class IX kappa light 
chain, the germline sequence for clone A9 (coded 21 A [39]) is different from clones A124 and C35: these clones use, like the previously described hLH cross-reactive clones, the germline

(A)

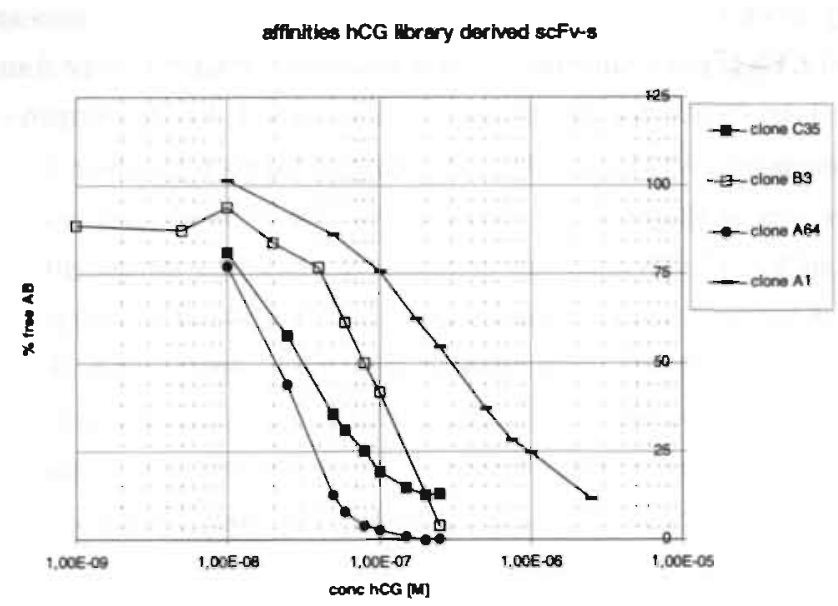

(B)

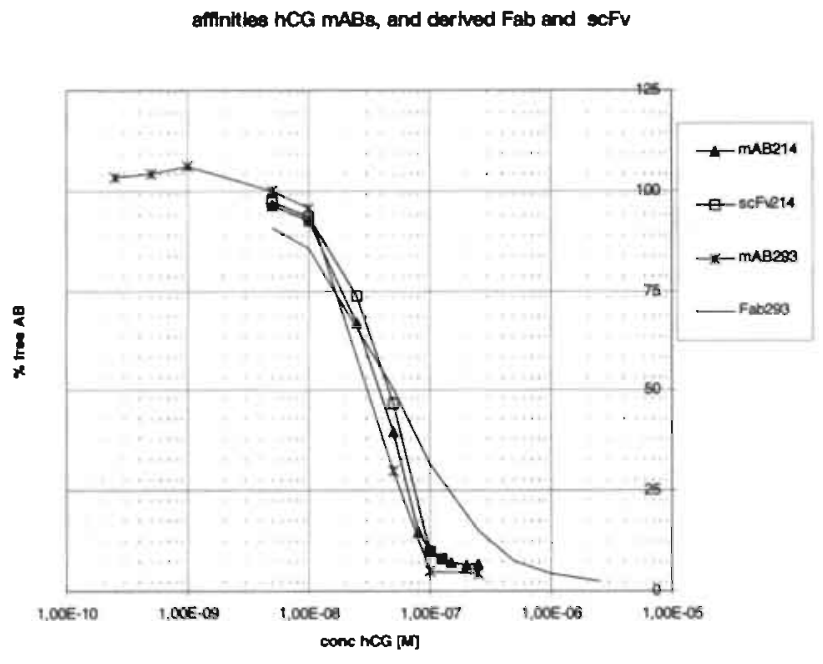

Figure 4. Affinity plots from scFv's (panel A) and mABs (panel B). The percentage of free $A B$ was plotted against the antigen concentration (logarithmic scale). The amount of free $A B$ (ie. not bound to soluble $\mathrm{Ag}$ ) was determined on BlAcore and related to the input amount $\mathrm{AB}$ (no $\mathrm{Ag}$ added). The $K_{d}$ was derived from the plots at $50 \%$ binding. 
sequence encoded $21 \mathrm{C}$. The ten-fold lower affinity found for clone $\mathrm{A} 9$, relative to the two other hCG specific clones, must be caused by the use of a different light chain germline sequence.

\section{Discussion}

\subsection{Molecular analysis of the murine antibody response against $h C G$}

For the evaluation of the immune response against hCG, we studied the early immune response by injection of mouse \# 1 with a single dose of antigen, while the effect of maturation was examined with mouse $\# 2$ by an additional boost with a decreased amount of antigen. From

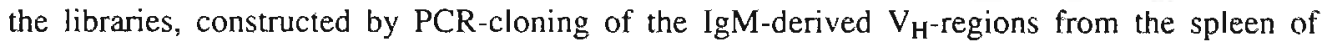
mouse \#1 (coded library M1), and of the IgG-derived $\mathrm{V}_{\mathrm{H}^{-}}$-regions from mouse \#I and \#2 (coded library GI and G2 respectively), a spectrum of antibodies with different specificities was selected with intact hCG (Table 2). From the Gl-library a group of $\beta$-cf specific, hLH crossreactive antibodies were retrieved, and three $\beta$-hCG-specific antibodies (also $\beta$-cf specific), while the $\mathrm{M} 1$ repertoire, made from the same mouse, yielded only anti $\alpha$-unit antibodies. The G2-repertoire, constructed from mouse \#2, gave an even "more" diverse set of specificities, including a different $\beta$-unit specific, hLH cross-reactive antibody, two $\alpha$-unit specific AB's, and one hLH cross-reactive antibody, recognising the holo-form of the hormone.

Examination of the sequences (Table 1) shows a correlation between specificity of the clones and the subgroup of the heavy chain variable region, and to some degree with the length of the $\mathrm{V}_{\mathrm{H}}$ CDR3 (Table 1). This would imply that the heavy chain variable region would dominate the specificity, a phenomenon observed before [40]. All anti $\beta$-unit scFv's are paired with only one family of light chain V-regions, which might be an indication for maintenance of the pairing originally present in the B-cell produced antibodies [41]. This phenomenon was also found in the group of $\alpha$-unit specific scFv's: all, except A7, pair with a family XVII light chain. even though the antibodies originate from different mice. Within the group of hCG-specific antibodies, one of the clones (A9) uses another (although related) light chain variable region derived from a different gemmline sequence (21A) as the other group members C35 and A124 (derived from germline analogue $21 \mathrm{C}$ ). The specificity of clone A9 was not changed, but its affinity was completely altered by the use of the other $V_{L}$ : the $s c F v$ produced by this clone showed to have a ten-fold lower affinity. Apparently the $V_{L}$ aids in the formation of optimal interactions with the antigen and thereby influences the strength of binding; this principle actually forms the basis for light chain shuffling [42] for improving the affinity without changing the specilicity.

Comparison of the sequences of the $\beta$-unit, hLH cross-reactive scFv's selected from library Gl with those from our panel of monoclonal antibodies (Table 1) shows a remarkable resemblance in the used $\mathrm{V}_{\mathrm{H}}$-family: both the library derived as well as the hybridoma produced, 
$\beta$-unit specific antibodies (mAB's 147,212 and 214) use $V_{H}$ family VII, although originating from different, but closely related germline genes (37A11 and VH105 for the library and hybridoma derived antibodies respectively). The light chain found in the $\beta$-unit specific scFv's is closely related to the one from $\mathrm{mAB} 147$ : the germline genes used are $21 \mathrm{C}$ for the library

Table 2. Characteristics of the hCG reactive scFvs selected from libraries and hybridoma produced $\mathrm{mABs}$.

\begin{tabular}{|c|c|c|c|c|c|c|c|c|c|}
\hline \multirow{2}{*}{$\begin{array}{l}\text { Format } \\
A b\end{array}$} & \multirow[t]{2}{*}{ Name } & \multirow[t]{2}{*}{ Library } & \multicolumn{2}{|c|}{ Selection method } & \multicolumn{2}{|c|}{$\beta$-chain } & \multirow[t]{2}{*}{$\beta$-core } & \multirow{2}{*}{$\begin{array}{c}\alpha / \beta \text {-chain } \\
(\mathrm{CG} / \mathrm{L} / \mathrm{H})\end{array}$} & \multirow[t]{2}{*}{$a$-chain } \\
\hline & & & Soluble Ag & Panning & CG & LH & & & \\
\hline \multirow{15}{*}{ scFy } & $\mathrm{Al}$ & G1 & $\mathrm{x}$ & & + & + & + & - & - \\
\hline & As & GI & $\mathrm{x}$ & & + & + & + & - & - \\
\hline & A9 & GI & $x$ & & + & - & + & - & - \\
\hline & All & $\mathrm{Gl}$ & & $x$ & + & + & + & - & - \\
\hline & A61 & Gl & & $\mathrm{x}$ & + & + & + & - & - \\
\hline & A 64 & GI & & $\mathrm{x}$ & + & + & + & - & - \\
\hline & $\mathrm{CHI}$ & GI & & $x$ & + & + & + & - & - \\
\hline & A124 & $\mathrm{Gl}$ & & $x$ & + & - & + & - & - \\
\hline & 035 & GI & & $x$ & + & - & + & - & - \\
\hline & $A s$ & MI & & $\mathrm{x}$ & - & - & - & - & + \\
\hline & A7 & M1 & & $x$ & - & - & - & - & + \\
\hline & B3 & G2 & $\mathrm{x}$ & & - & - & - & + & - \\
\hline & B4 & G2 & $\mathrm{x}$ & & + & + & ? & - & - \\
\hline & B6 & G2 & $\mathrm{x}$ & & - & - & - & - & + \\
\hline & B7 & $G_{2}$ & $\mathrm{x}$ & & - & - & - & - & + \\
\hline $\mathrm{mAB}$ & 212 & & & & + & + & + & - & - \\
\hline scFv & 212 & & & & + & + & + & - & - \\
\hline $\mathrm{mAB}$ & 214 & & & & + & + & + & - & - \\
\hline scFv & 214 & & & & + & + & + & - & - \\
\hline $\mathrm{mAB}$ & 293 & & & & + & - & - & - & - \\
\hline Fab & 293 & & & & + & - & - & - & - \\
\hline
\end{tabular}

derived antibody and $21 \mathrm{~A}$ for the hybridoma derived antibody, and the length of the CDRI is identical also. ScFv B4 with a $V_{H}$ family $X$, derived from library $G 2$, might recognise a different epitope on the $\beta$-subunit as the $V_{H}$ family VII containing scFv's, selected from library $G 1$.

The above described analogy between library and hybridoma derived anti $\beta$-cf antibodies in respect to the used heavy chain $V$-region was not found for the $\alpha / \beta$-heterodimer specific $A B s$ : scFv B3 and mAB 121 use different $\mathrm{V}_{\mathrm{H}^{-}}$-families.

\subsection{Analysis of somatic mutations}

The best case for studying the effect of maturation is formed by the group of $\alpha$-unit specific antibodies, the only group of antibodies selected from the $\mathrm{M} l$ library, representative for the early immune response, and from the G2-library, constructed from the boosted mouse and hence illustrative for the late response. The alignment of the heavy chain amino acid sequences (Table 
I) with the germline analogue $\mathrm{C} 57 \mathrm{G} 26$ reveals a high number of somatic mutations in the library G2 derived clone B7, especially when compared with the IgM-derived clones of mouse \#1. The IgM derived clones A6 and A7 both contain 6 mutated amino acid residues, whereas clone B7 has 15 mutated residues within a stretch of 84 amino acids. As was suggested in literature [43, 44], analysis of the nucleotide sequence should give additional information, since an increased ratio of replacement compared to silent mutations is typical for somatically mutated CDRs. Apparently FRs do not tolerate most replacement mutations, as a consequence of structural constraints. Indeed, clone B7 contains as many as 32 mutations in its heavy chain V-region with most replacement mutations in its CDR 1 and CDR2 (3 and 7 mutated nucleotides, see Table 3 ).

Table 3. Distribution of codons with replacement and silent mutations in VH- and VLgenes of library derived and hybridoma produced antibodies.

library specificity

Ratio Replacement vs. Silent Mutations (R/S)

total \# mut

\begin{tabular}{|c|c|c|c|c|c|c|c|c|c|c|}
\hline & & & & FR1 & CDR1 & FR2 & CDR2 & FR3 & CDR3 & \\
\hline \multirow[t]{2}{*}{ scFv B3 } & $\mathrm{G} 2$ & $\alpha / \beta$ & VH & $1 / 2$ & $0 / 1$ & $2 / 0$ & $3 / 1$ & $0 / 1$ & $x$ & 11 \\
\hline & & & VL & $7 / 0$ & $5 / 1$ & $0 / 0$ & $0 / 0$ & $0 / 0$ & $0 / 0$ & 13 \\
\hline \multirow[t]{2}{*}{ scFv B6 } & G2 & $\alpha$ & VH & $1 / 1$ & $1 / 0$ & $3 / 0$ & $3 / 1$ & $0 / 2$ & $x$ & 12 \\
\hline & & & VL & $0 / 1$ & $0 / 1$ & $0 / 0$ & $0 / 0$ & $1 / 3$ & $5 / 0$ & 11 \\
\hline \multirow[t]{2}{*}{ scFv B7 } & $\mathrm{G} 2$ & $\alpha$ & $\mathrm{VH}$ & $5 / 3$ & $3 / 2$ & $5 / 2$ & $7 / 0$ & $2 / 3$ & $x$ & 32 \\
\hline & & & VL & $0 / 1$ & $3 / 0$ & $0 / 0$ & $1 / 0$ & $1 / 3$ & $5 / 0$ & 14 \\
\hline \multirow[t]{2}{*}{$S c F \vee A 6$} & M1 & $\alpha$ & $\mathrm{VH}$ & $1 / 0$ & $1 / 0$ & $2 / 1$ & $2 / 2$ & $1 / 1$ & $x$ & 17 \\
\hline & & & VL & $2 / 1$ & $3 / 0$ & $0 / 0$ & $3 / 0$ & $3 / 1$ & $5 / 0$ & 18 \\
\hline \multirow[t]{2}{*}{ scFv $A 7$} & M1 & $\alpha$ & VH & $0 / 0$ & $1 / 0$ & $1 / 1$ & $4 / 2$ & $0 / 3$ & $x$ & 12 \\
\hline & & & VL & 080 & $0 / 0$ & $0 / 0$ & $0 / 0$ & $0 / 0$ & $1 / 0$ & 1 \\
\hline \multirow[t]{2}{*}{ scFv A9 } & G1 & $\beta-C G$ & VH & $2 / 2$ & $2 / 0$ & $0 / 0$ & $4 / 1$ & $2 / 0$ & $x$ & 13 \\
\hline & & & VL & $0 / 0$ & $8 / 1$ & $1 / 0$ & $4 / 1$ & $0 / 0$ & $0 / 1$ & 16 \\
\hline \multirow[t]{2}{*}{ scFv C11 } & G1 & $\beta-C G / L H$ & VH & $0 / 2$ & 010 & $0 / 0$ & $1 / 0$ & $3 / 1$ & $x$ & 7 \\
\hline & & & VL & $0 / 0$ & $0 / 0$ & $0 / 0$ & $0 / 0$ & $0 / 1$ & $1 / 1$ & 3 \\
\hline \multirow[t]{2}{*}{ scFv B4 } & G2 & $\beta-\mathrm{CG} / \mathrm{LH}$ & VH & $1 / 1$ & $0 / 0$ & $3 / 0$ & $3 / 0$ & $2 / 0$ & $x$ & 10 \\
\hline & & & $\mathrm{VL}$ & $0 / 0$ & $0 / 0$ & $0 / 0$ & $1 / 1$ & $1 / 2$ & $0 / 0$ & 5 \\
\hline \multirow[t]{2}{*}{$\mathrm{mAB} 147$} & & $\beta-C G / L H$ & VH & $4 / 3$ & $4 / 0$ & $1 / 1$ & $11 / 1$ & $4 / 2$ & $x$ & 39 \\
\hline & & & VL & $1 / 1$ & $2 / 1$ & $0 / 0$ & $0 / 0$ & $0 / 1$ & $1 / 1$ & 8 \\
\hline \multirow[t]{2}{*}{$\mathrm{mAB} 212$} & & $\beta-C G / L H$ & VH & $2 / 6$ & $7 / 1$ & $1 / 1$ & $8 / 4$ & $3 / 1$ & $x$ & 34 \\
\hline & & & VL & $4 / 2$ & $3 / 1$ & $1 / 0$ & $0 / 0$ & $2 / 1$ & $5 / 2$ & 21 \\
\hline \multirow[t]{2}{*}{$\mathrm{mAB} 121$} & & $\alpha / \beta$ & VH & $1 / 1$ & $0 / 0$ & $0 / 0$ & $0 / 0$ & $0 / 0$ & $x$ & 2 \\
\hline & & & VL & $0 / 1$ & $0 / 0$ & $0 / 0$ & $0 / 0$ & $1 / 1$ & $0 / 0$ & 3 \\
\hline
\end{tabular}


For comparison, the IgM derived clone A6 carries 11 mutations with one and two replacement mutations in CDRI and CDR2 respectively, and clone A7 has 12 mutated nucleotides with one and four replacement mutations for $\mathrm{CDR} 1$ and $\mathrm{CDR} 2$ respectively. By an additional immunisation with a low amount of hCG, the process of somatic mutation and antigen selection serms to be focused on CDR1 and 2. Although scFv B6 has a high aftinity ( $35 \mathrm{nM})$, we do not know if the mutations of the $\mathrm{V}_{\mathrm{H}} \mathrm{CDR}$ 1- and CDR2-residues really contribute to an improvement of the affinity, because those from scFv's A6 and A7 were not determined. Our data confirm the rule of the increased ratio replacement to silent mutations for the CDRs, both for phage library derived antibodies as well as hybridoma derived antibodies (Table 3). The light chain regions of antibodies from both sources have less mutations (in nucleotide and amino acid sequence) as the heavy chain regions, with some exceptions.

A comparison between antibodies selected from the G1-library with those from the G2library. and with different specificities, does not seem to be useful as can be concluded by examination of the hybridoma derived antibodies. MAB 121, a representative for the late

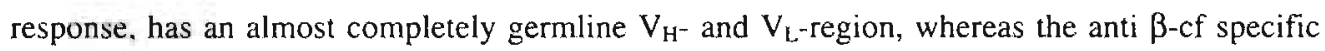

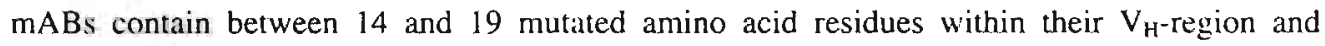
between three and 12 mutated residues within their $V_{\mathrm{L}}$-region. A comparable low level of mutations were found in some of the high affinity clones (A64 and A61) within the group of the $\mathrm{hCG} / \mathrm{hLH}$ cross-reactive antibodies (three and four residues respectively). This is consistent with reports on the isolation of high affinity antibodies, just four days after exposure to antigen [45], and with the finding of high affinity antibodies containing germline sequences [46]. Within the group of the hCG/hLH cross-reactive antibodies, the $\mathrm{V}_{\mathrm{H}}$-region of one low affinity antibody (A1) seems to be heavily mutated, but it is remarkable to see that the mutations are only found within FRI and FR2. It might be possible that the $\mathrm{V}_{\mathrm{H}^{-}}$-region of this clone and clone $\mathrm{A} 5$ represent chimeric products resulting from a PCR crossover event [47]. The high degree of homology of the $5^{\prime}$ end of clones $\mathrm{Al}$ and $\mathrm{A} 5$ with the germline sequence $\mathrm{C} 57 \mathrm{C} 2$ would suggest a crossover with this V-region.

The effect of somatic mutation on antibody affinity could only be detected in the group of hCG/hLH cross-reactive scFv's. Clone C11, with a two- to three-fold lower affinity as clone A64, contains two mutated residues within $V_{H}$ CDR3 and one in $V_{L}$ CDR3. Remarkable is that this change is mediated by a relatively drastic substitution, i.e. in $V_{H}$ CDR3 of the negatively charged aspartic acid (residue 100) into the polar asparagine (and with the conservative change at position 100E: alanine into proline), in $\mathrm{V}_{\mathrm{L}} \mathrm{CDR} 3$ of aspartic acid (residue 94) to the nonpolar glycine. Within the group of hCG specific antibodies a dramatic difference in affinity can be observed between scFv A9 and the other members of this group, but this is caused by pairing with a different light chain family. 


\subsection{Possible diagnostic applications of the library derived scFv's}

As the major components of commercially available pregnancy tests, a large collection of murine monoclonal antibodies directed against the human chorionic gonadotropin has been built up over the last fifteen years in our laboratory at Organon Teknika. Thirty-two fusions were needed before a useful hCG-specific AB (coded 293) was obtained, due to the fact that most hybridomas produce hLH cross-reactive antibodies. We have explored phage display technology to determine if this selection-based procedure could be more efficient to select antibodies with unique hCG specificities.

From just two repertoires a complete range of scFv's with different specificities were readily selected on hCG coated onto a polystyrene surface, and with soluble biotinylated antigen. The majority of the clones selected from library Gl were directed against the $\beta$-cf and were hLH cross-reactive. As pepscan analysis revealed, these antibodies recognised peptides located in the aminoterminus, with the common sequence RCRPI (residue 8 to 12), also present in the $\beta$-cf. Except for isoleucine and cysteine, the area is completely surface exposed (with the side chains of the arginine residues being solvent exposed) and thereby accessible for interaction with antibodies. Interestingly, this epitope contains the two arginine residues, which have been implied to confer hCG-specificity to mABs LNN-hCG-2 and INN-hCG-32 [22].

Because of our interest for in vitro diagnostics, our attention focused on the group of hCG specific antibody fragments. The three hCG-specific scFv's coded A124 and C35, isolated from library $\mathrm{G} 1$ by biopanning, and $\mathrm{A} 9$, originated from the same library (G1), using in solution selection. No cross-reactivity against hLH (or hFSH) could be detected on Western blot or with surface plasmon resonance. The hFSH-CTP chimera was not detected by the antibody fragments with surface plasmon resonance, suggesting that the carboxyterminal part of the $\beta$-chain (residue number 115 to 145 ), unique for $\mathrm{hCG}$, is not recognised. Their anti $\beta$-cf reactivity confirmed the assumption, since this degradation product misses the C.P-part. From these data we concluded that the epitope must be located within residues 6 to 40 , or within the segment containing the residues 55 to 92 . Therefore it may be identical to the epitope of $\mathrm{mAB} B 112$ [22], in which the residue number 77 , asparagine, of the $\beta$-subunit confers hCG-specificity, or to the epitope mentioned before and recognised by mAB's INN-hCG-32 and INN-hCG-2 [48], for which the residues number 8 and 10 (both arginine) are involved in hCG specificity.

In respect to specificity, we believe that we have selected a number of scF v's, which might be useful for the development of a pregnancy assay. For capture of the intact heterodimer, one of the anti- $\alpha$-chain specific scFv's B6 or B7 could be used, or even better the anti- $\alpha / \beta$-heterodimer $s c F v B 3$. By increasing the avidity to $n=2$ or $n=4$ binding sites, the sensitivity might be enhanced $[49,50]$. As detection antibody one of the hCG specific scFv's A9, Al24 or C35 might be applied. To increase sensitivity, a dimeric format might be preferred, especially for obtaining a synergistic effect. If the hCG specific scFv's recognise the $\beta 1$ epitope from mABs INN-hCG-32 and INN-hCG-2, then it should be pussible to identify a synergistic (capture) $\alpha 2$ epitope 
recognising antibody [48], thereby increasing the "total" affinity constant and correlated with affinity the sensitivity of the immuno-assay [51, 52].

When the affinities of the selected antibody fragments were evaluated, we found no differences with monoclonal antibodies, which are currently applied in diagnostic assays. We therefore conclude, that the anti-hCG scFv's selected from the immune repertoires might form the basis for a new generation pregnancy assay, for which the specificity. the sensitivity and the incubation times can be manipulated by application of molecular engineering technologies (reviewed in $[53,54]$ ).

\section{References}

1) Lapthorn, A. J., Harris, D. C., Littlejohn, A., Lustbader, J. W., Canfield, R. E., Machin, K. J., Morgan, F. J. and Isaacs, N. W., Nature 1994. 369: 455.

2) Schroeder. H. R. and Halter, C. M., Clin. Chem. 1983. 29: 667.

3) Kato, Y. and Braunstein. G. D., J. Clin. Endocrinol. Metab. 1988. 66: 1197.

4) Cole, L. A., Clin. Chem. 1997. 43: 2233.

5) Krichevsky, A., Armstrong, E. G., Schlatterer, J., Birken, S., O'Connor, J., Bikel. K., Silverberg. S., Lustbader, J. W. and Canfield, R. E., Endocrinology 1988. 123: 584.

6) Masure, H. R., Jaffee, W. L., Sickel, M. A., Birken, S., Canfield, R. E. and Vaitukaitis, J. L., J. Clin. Endocrinol. Metab. 1981. 53: 1014.

7) Papapetrou, P. D., Sakarelou, N. P., Braouzi, H. and Fessas, P., Cancer 1980. 45: 2583.

8) Clackson, T., Hoogenboom, H. R., Griltiths, A. D. and Winter, G., Nature 1991. 352: 624.

9) Canfield, R. E. and Morgan, F. J., in Berson. S. A. and Yalow. R. S. (Eds.). Methods in investigative and diagnostic endocrinology, North Holland Publishing Co., Amsterdam 1973, p. 727.

10) Chirgwin, J. M., Przybyla. A. E., MacDonald, R. J. and Rutter. W. J., Biochemistry 1979. 18: 5294.

11) de Haard, J. J. W., Kazemier, B., van der Bent, A., Oudshoom, P., Boender, P., van Gemen, B., Arends, J.-W. and Hoogenboom, H. R. M., Protein Eng. 1998. 11: 1267.

12) de Haard, J. J. W., Kazemier, B., Koolen, M. J. M., Nijholt, L. J., Meloen, R. H., Van Gemen, B., Hoogenboom, H. R. and Arends, J.-W.. Clin. Diagn. Lab. Immumol. 1998. 5: 636.

13) Hoogenboom. H. R., Griffiths, A. D., Johnson, K. S., Chiswell, D. J., Hudson, P. and Winter, G., Nucleic Acids Res. 1991. 19: 4133.

14) Hopp. T. P., Prickett, K. S., Price, V. L., Libby, R. T., March. C. J.. Cerretti, D. P., Urdall, D. L. and Conlon. P. J., BioTechnology (NY) 1988. 6: 204.

15) Dower, W. J., Miller, J. F. and Ragsdale, C. W.. Nucleic Acids Res. 1988. 16:6127. 
16) Marks, J. D., Hoogenboom. H. R., Bonnert, T. P., McCafferty, J., Griffiths, A. D. and Winter, G., J. Mol. Biol. 1991. 222: 581.

17) Hawkins, R. E., Russell, S. J. and Winter, G.. J. Mol. Biol. 1992. 220: 889.

18) de Haard, J. J. W., Kazemier, B., Oudshoorn, P., Boender, P., van Gemen, B., Koolen, M. J. M., van der Groen, G., Hoogenboom, H. R. M. and Arends, J.-W., J. Gen. Virol. 1998. 79: 2883.

19) Griffiths, A. D., Malmqvist, M., Marks, J. D., Bye, J. M., Embleton, M. J., McCafferty, J., Baier, M., Holliger, K. P., Gorick, B. D., Hughes, J. N., Hoogenboom, H. R. and Winter, G., EMBO J. 1993. 12: 725.

20) Matzuk, M. M., Spangler, M. M., Camel, M., Suganuma, N. and Boime, I., J. Cell. Biol. 1989. 109: 1429.

21) Muyan, M., Furuhashi, M., Sugahara, T. and Boime, I., Mol. Endocrinol. 1996. 10: 1678.

22) Moyle, W. R., Matzuk, M. M., Campbell, R. K.. Cogliani, E., Dean-Emig. D. M., Krichevsky, A., Barnett, R. W. and Boime, I., J. Biol. Chem. 1990. 265: 8511.

23) Berger, P., Schwarz, S., Spottl, G., Wick, G. and Mann, K., J. Clin. Endocrinol. Metab. 1993. 77: 347.

24) Geysen, H. M., Meloen, R. H. and Barteling, S. J., Proc. Natl. Acad. Sci. U.S.A. 1984. 81: 3998.

25) Kazemier, B., de Haard, H., Boender, P., van Gemen, B. and Hoogenboom, H. R.. .I. Immunol. Methods 1996. 194: 201.

26) Birken, S., Krichevsky, A., O'Connor, J., Lustbader, J. and Canfield. R., Glycoprotcin Hormones: structure, synthesis and biologic function., Serono Symposia 1990.

27) Nieba, L., Krebber, A. and Pluckthun, A., Anal. Biochem. 1996. 234: 155.

28) Fares, F. A., Suganuma, N., Nishimori, K., LaPolt, P. S., Hsueh, A. J. and Boime, I., Proc. Natl. Acad. Sci. U.S.A. 1992. 89: 4304.

29) Seidman, J. G., Leder, A., Edgell, M. H., Polsky, F., Tilghman. S. M., Tiemeier, D. C. and Leder, P., Proc. Nall. Acad. Sci. U.S.A. 1978. 75: 3881.

30) Crews, S., Griffin, J., Huang, H., Calame, K. and Hood, L., Cell 1981. 2.5: 59.

31) Joho, R., Gershenfeld, H. and Weissman, I. L., EMBO J. 1984. 3: 185.

32) Sanz, I. and Capra, J. D., Proc. Natl. Acad. Sci. U.S.A. 1987. 84: 1085.

33) Puisieux, A., Bellet. D., Troalen, F., Razafindratsita, A., Lhomme, C., Bohuon, C. and Bidart, J. M., Endocrinology' 1990. 126: 687.

34) Cole, L. A., Kardana, A., Andrade-Gordon, P., Gawinowicz, M. A., Morris, J. C., Bergert. E. R., O'Connor, J. and Birken, S., Endocrinology 1991. 129: 1559.

35) Wang, H., Segal, S. J. and Koide, S. S., Endocrinology 1988. 123: 795.

36) Nahmias, C., Strosberg, A. D. and Emorine, L. J., J. Immunol. 1988. 140: 1304.

37) Heinrich, G., Traunecker, A. and Tonegawa, S., J. Exp. Med. 1984. 159: 417. 
38) Foster, M. H., Sabbaga, J., Line, S. R., Thompson, K. S., Barrett, K. J. and Madaio, M. P., J. Immunol. 1993. 151: 814.

39) Alanen, A. and Weiss, S., Eur. J. Immunol. 1989. 19: 1961.

40) Graus, Y. F., de Baets, M. H., Parren, P. W., Berrih-Aknin, S., Wokke, J., van Breda Vriesman, P. J. and Burton, D. R., J. Immunol. 1997. 158: 1919.

41) Gherardi, E. and Milstein, C., Nature 1992. 357: 201.

42) Marks, J. D., Griffiths, A. D., Malmqvist, M., Clackson, T. P., Bye. J. M. and Winter, G., Bio/Technology 1992. 10: 779.

43) Berek, C. and Milstein, C., Immunol. Rev. 1987. 96: 23.

44) Dorner, T., Brezinschek, H. P., Brezinschek, R. I., Foster, S. J., Domiati-Saad, R. and Lipsky, P. E.. J. Immunol. 1997. 158: 2779.

45) Newman, M. A., Mainhart, C. R., Mallett, C. P., Lavoie, T. B. and Smith-Gill, S. J., J. Immunol. 1992. 149: 3260.

46) Roost, H. P., Bachmann, M. F., Haag, A., Kalinke, U., Pliska, V., Hengartner. H. and Zinkernagel, R. M., Proc. Natl. Acad. Sci. U.S.A. 1995. 92: 1257.

47) Meyerhans, A., Vartanian, J. P. and Wain, H. S., Nucleic Acids Res. 1990. 18: 1687.

48) Klonisch, T., Delves. P. J., Berger, P., Panayotou, G., Lapthorn, A. J., Isaacs, N. W., Wick, G., Lund, T. and Roitt, I. M., Eur. J. Immunol. 1996. 26: 1897.

49) Lindner, P., Bauer, K., Krebber, A., Nieba, L., Kremmer, E., Krebber, C., Honegger, A., Klinger, B., Mocikat, R. and Pluckthun, A., Biotechniques 1997. 22: 140.

50) Gandecha, A., Owen, M. R., Cockburn, W. and Whitelam, G. C., Protein Expr. Purif. 1994. 5: 385 .

51) Lew, A. M., J. Immunol. Methods 1984. 72: 171.

52) Devey, M. E. and Steward, M. W., in Kemeny, D. M. and Challacombe, S. J. (Eds.), ELISA and Other Solid Phase Immunoassays., John Wiley \& Sons Ltd, London 1988, p. 135.

53) Hoogenboom, H. R., Trends Biotechnol. 1997. 15: 62.

54) de Haard, H., Henderikx, P. and Hoogenboom, H. R., Adv. Drug Rev. 1998. 31: 5.

\section{Acknowledgments}

We wish to thank Bert van der Weijer for purified hCG. Tom Oosterlaken and Mirjam Klein-Rot for preparation of the splecus, and Adrialan de Bruïne and Simon Hufton for critical reading of the manusicript. Finally, the Dutch Ministry of Economic Affairs for the financial support (PBTS grant BIO 91028). 


\section{Chapter 5}

\section{SELECTION OF MURINE ANTI- HIV-1 P24 ANTIBODY FRAGMENTS}

Published as

Selection of recombinant, library derived antibody fragments against p24 for Human Immunodeficiency Virus type 1 diagnostics

Hans J.W. de Haard, Bert Kazemier, Marck J.M. Koolen, Liekle J. Nijholt, Rob H. Meloen, Bob van Gemen, Hennie R.J.M. Hoogenboom and Jan-Willem Arends

Clinical and Diagnostic Laboratory Immunology

1998; Volume 5, No. 5; pages 636 - 644 


\section{Abstract}

By application of combinatoria] library technology, we generated the first recombinant antibody fragments directed against the major capsid protein p24 of Human Immunodeficiency Virus type 1 (HIV-1). A library of single-chain Fv fragments (scFvs) was constructed by using the antibody variable region genes of B-cells derived from a viral lysate-immunized mouse. Antibodies were selected by panning or by enrichment with biotinylated antigen, yielding four different families of antibody fragments. The different types of scFvs were characterised by affinity measurements, by antigen recognition on Western blots, and by pepscan analysis. The epitope of one of the scFvs is located near the residues involved in CypA binding, thereby making it an attractive candidate for therapeutic applications. Comparison of the Variable region (V-) gene sequence of a previously described monoclonal antibody reactive against this immunodominant epitope revealed the usage of the identical combination of $V_{H}$ and $V_{K}$ regions. Thus, this is one of the rare examples in which the original combination in a library-derived antibody fragment was retrieved. After appropriate affinity and format improvements, the best of our recombinant scFvs may form the basis for a sensitive p24 assay as a measure of viral load. In addition, anti-p24 scFvs could be expressed as intracellular antibodies (intrabodies) to aid in the treatment of HIV infections.

\section{Introduction}

Human Immunodeficiency Virus (HIV) infection is diagnosed by detecting virus-specific antibodies (Ahs). or the virus itself, by means of p24 antigen (Ag) detection or by quantitative amplification procedures such as PCR (38) or nucleic acid sequence-based amplification (NASBA) (62), or by coculturing and subsequent virus detection procedures. During a diagnostic window of 6 to 8 weeks after infection, antibodies to HIV are undetectable. and alternative diagnostic methods would help to reduce the residual risk of transfusion transmission of HIV. Recently, the Food and Drug Administration recommended the implementation of p24 antigen tests in donor screening (20). The p24 capsid protein forms the viral core containing the singlestranded RNA genome and it is abundantly present in the virus particle. Besides the structural role of the protein in forming the core of the mature virion, the molecule is essential during viral assembly; it plays a pivotal role in viral penetration or uncoating or both, a function which may be mediated by binding of p24 to the human cellular proline rotamase cyclophilin $A(4,41,60)$. With current enzyme-linked immunosorbent assays (ELISAs), the presence of p24 Ag may be assessed 5 to 14 days earlier than could an Ab response measured by anti-HIV type 1 (anti-HIV1) or anti-HIV-2 enzyme immunoassays $(8,9,66)$. In addition, the capsid protein may be considered a marker for virus replication $(3,26,65)$, and its detection in an extremely sensitive 
immunoasisay would offer a cheap and generally applicable alternative to PCR-based assays for the diagnosis of reactivation during treatment of HIV-1 infected patients with (combinations of) nucleoside reverse transcriptase inhibitors or protease inhibitors $(59,19,64)$. When reactivation, as the result of the evolution of resistant HIV mutants, is detected, treatment may be changed to other drugs. Rapid and sensitive assays that can carefully detect the presence of p24 in serum are therefore crucial for early detection and monitoring of viral replication (66).

The sensitivity and specificity of the presently used anti-p24 immunoassays are limited by the aflinity of the monoclonal Abs (MAbs) used for capturing and/or detection of the $\mathrm{Ag}$, although by signal amplification in combination with heat denaturation, the sensitivity can be increased to the level obtained by PCR (6). The availability of the Ab genes in recombinant antip24 Abs allows the improvement of affinity by mutagenesis methods, as well as the engineering of avidity, thereby helping to improve the sensitivity of early virus detection. During in vivo maturation, the obtained affinities are limited by the off-rate, i.e., the rate at which the $\mathrm{Ab}-\mathrm{Ag}$ complex dissociates. The off-rate of in vivo-matured Abs is in the order of $10^{-3}$ to $10^{-4} \mathrm{~s}^{-1}$, which permits endocytosis of membrane bound antibody-antigen complexes on B-cells (21). However, in vitro maturation with phage display allows the selection of Abs with lower off-rates, leading to affinities in the picomolar range $(1,57)$. Besides the obvious diagnostic application, it may also be possible to use anti-p24 single-chain Fv fragments (scFvs) for therapy. By expression with a retention signal for the endoplasmatic reticulum, the scFvs may interfere with virus assembly in the infected cells, as was demonstrated with anti-gp120 (12) and anti-'Tat (45) Abs. For intracellular expression, even murine Abs might be applied in humans: a human anti-mousc antibody (HAMA) response can not be induced, since the fragments are shielded from the immune system.

In this context, we aimed to isolate anti-p24 scFvs from a phage Ab library made from a mouse immunized with HIV-] viral lysate. Affinities were determined with surface plasmon resonance and compared with those of MAbs currently used in p24 assays. The specificity of the selected Ab fragments was determined by ELISA, Western blotting, and pepscan analysis. One of the recombinant Ab fragments recognized an epitope, which was also detected by a previously described and sequenced murine Mab. Both the recombinant $\mathrm{Ab}$ and the hybridoma-derived $\mathrm{Ab}$ have nearly identical heavy and light chain variable regions, and, as such, the selected scFv D2 is one of the first examples in which the original pairing of both $\mathrm{V}_{\mathrm{H}}$ and $\mathrm{V}_{\mathrm{L}}$ was maintained, in spite of the very low probability of finding this in a combinatorial library (23).

These newly described anti-p24 recombinant scFvs offer a starting point for Ab affinity maturation and engineering (for review see $(30,67,68)$ ), and may be useful as intracellular Abs (intrabodies) for HIV therapy. 


\section{Materials and methods}

\subsection{Library construction}

$\mathrm{A} \mathrm{Balb} / \mathrm{c}$ mouse was immunized with $100 \mu \mathrm{g}$ of protein from a viral lysate, which had been prepared from infected $\mathrm{H} 9$ cells (52) and purified by ultracentrifugation, plus Freunds complete adjuvant. After 6 weeks, a booster with $100 \mu \mathrm{g}$ lysate proteins plus incomplete adjuvant was administered. Three days later, the animal was sacrificed and the spleen was removed. Upon extraction of the spleen, $20 \mu \mathrm{g}$ of total RNA was isolated (13) and $5 \mu \mathrm{g}$ of this was transcribed into random-primed cDNA.

The immunoglobulin $\mathrm{Gl}$ ( $\mathrm{IgGl}$ )-derived $\mathrm{V}_{\mathrm{H}^{-}}$and $\mathrm{V}_{\mathrm{K}}$-encoding DNA fragments as well as the linker fragment (the latter was cloned in pUC19 by using synthetic oligos, which encoded the 15-residue linker described by Huston and colleagues (33)) were obtained by amplification (Amplitaq; Perkin-Elmer Cetus) with the primers (14) listed in Table 1. The fragments were purified from an agarose gel with the QIAEX kit (Qiagen, Hilden, Germany), and assembled in splicing by overlap extension-PCR (32).

The assembled scFv-encoding DNA fragments were also purified from the gel, digested with Sfil and NotI (Pharmacia, Lund, Sweden), and cloned into the phagemid vector pV1, which is identical to pHEN1 (31) but with a FLAG-tag (53) instead of a Myc-tag. Transformations were performed by clectroporation in JM101 (17). The library consisted of $10^{7}$ clones, of which 60 to $70 \%$ contained a complete insert as was deduced from PCR analysis.

\subsection{Selection of libraries}

The rescue of phagemid particles was performed as was described before (43). The capsid protein p24, expressed as full-length product in Escherichia coli under control of the inducible lac promoter (constructed at Organon Teknika [3a]) and encoding the HIV-1 strain RF-sequence, was affinity purified with MAb 39B (generated at Organon Teknika [unpublished data]). A sixwell tissue culture plate (Costar, Cambridge MA., USA) was coated with Ag at a concentration of $3 \mu \mathrm{g} / \mathrm{ml}$ in $50 \mathrm{mM} \mathrm{NaHCO} \mathrm{pH}_{3}$.6. Selection, washing, and elution were performed as described elsewhere (43).

For the construction of a light chain shuffling library of clone D2, the $V_{H}$ segment was amplified on plasmid DNA. The $V_{H}$ fragment was recombined with the linkers and the corresponding kappa light chain fragments, initially used for the generation of the library, yielding the shuffling library containing $10^{6}$ clones. Selection was performed with p24 biotinylated with $\mathrm{N}$-hydroxy-succinimide-biotin (Pierce, Rockford, Ill.); phage Ag complexes were captured with streptavidin-conted paramagnetic beads (Promega, Madison, Wis.). 
Table 1. Oligonucleotide primers used for construction of the library and sequencing

purpose primer name

VHlback (a) 5'aataggccagceggccatggcc(C/G)AGGTTCA(A/G)(G/C)TGCAGCAG(C/T)CTGG3

VH2back (a) 5'aataggeccagceggccatggecGAGGTG(C/A)AGCTT(G/C)T(G/C)GAGTCTGG3'

VH VH3back (a) 5'aataggcecagceggecatggecCCGA(G/T)GTGCAGCTTCAGGAGTCAGG3'

VHfor

IgG1-CHfor

VKlback

VK2back

VK3back

VK VK4back

VK5back

VKfor (a)

CKfor

VHLINKback 5'GGTCACCGTCTCCTCAgg3'

VK1LINKfor 5'AG(A/T)CTGAGTCATCT(G/T)GATGTCagatce3'

linker

VK2LINKfor

VK3LINKfor

VK4LINKfor

VK5LINKfor

geneIII

sequencing linkfwd

linkrev $5^{\prime}$ CCTGAGGAGACGGTGACC (A/G/C)(G/T)GG(G/T)(C/T)CC3'

5'TGGATAGACAGATGGGGG3'

5'ggatctGACATC(C/A)AGATGACTCAG(T/A)CT3'

5'ggatctGATATTGTGATGACGCAG(G/A)(C/A)T3'

5'ggatct(G/A)ACATTGTG(C/A)TGTCACAGTCTCC3'

5'ggatctGAAA(A/T)T(G/T)TTCTCACCCAGTCTCC3'

5'ggatctGATATT(G/T)TGATGACGCAG(C/T)CTGC3'

5 aatggaattccggcggccgcCCGTTTTA(T/G)(T/C)TCCAGCTT/G/T)GT(G/C)CC3'

5'AGTTGGTGCAGCATCAGCC3'

5'CTGCGTCATCACAATATCagatc:3'

5'GGAGACTGTGACA(G/T)CACAATGT(C/T)agatcc 3'

5'GGAGACTGGGTGAGAA(C/A)A(T/A)TTTCAGATagatce3'

5'GCAG(G/A)CTGCGTCATCA(C/A)AATATCagatcc3'

\section{5'TGAATTTTCTGTATGAGG3'}

5 . CTCGGGCGGTGGTGGGTC3'

5 ' GACCCACCACCGCCCGAG3'

${ }^{a}$ The Sfil sites in the VHback primer sequences and the Not site in the VKfor primer sequence are underlined. Nucleotides represented as upperease characters are carried by the $\mathrm{V}$ gene; those in lowercase characters are carried by the vector.

\subsection{Screening and sequencing of clones}

Soluble scFv was produced from individual clones by growth in V-shaped microtiter plates in 2*TYE medium as described before (43). The described method was applied for constructs transformed in the nonsuppressor strain TOP F', as well as in the suppressur strain JM101. Suppression is incomplete and results in leakage of free scFv in the culture medium, while the expression of genellI makes the cellular membranes more permeable (5), leading to higher concentrations of $\mathrm{Ab}$ fragments in the medium. Microtiter plates were coated with $3 \mu \mathrm{g}$ of recombinant p24 per $\mathrm{ml}$ (see above) or $5 \mu \mathrm{g}$ of viral Jysate per $\mathrm{ml}$ in $50 \mathrm{mM} \mathrm{NaHCO} 3$ (pH 9.6) for $16 \mathrm{~h}$ at room temperature. The plates were blocked for $2 \mathrm{~h}$ at room temperature with bovine serum albumine (BSA; $0.2 \%$ [wt/vol] in $0.1 \mathrm{M}$ Tris [pH 7.4]-30 mM KI). The culture 
supernatant was diluted fivefold in sample diluent (phospate-buffered saline [PBS], $20 \%$ normal goat serum; $1.1 \%$ Triton X-100). After incubation at room temperature for $2 \mathrm{~h}$, the plates were washed four times with PBS-Tween $20(0.05 \%)$, and bound scFv was detected with a mixture of a 1/4,000 dilution of anti-FLAG Ab M2 (Kodak IBI, New Haven, Conn.), and a 1/2,000 dilution of anti-mouse Ab-horseradish peroxidase conjugate (DAKO, Glostrup, Denmark). Following a l-h incubation and washing as before, staining was performed with tetramethylbenzidine and ureaperoxide as the substrate and stopped by adding an equal volume of $1 \mathrm{M} \mathrm{H}_{2} \mathrm{SO}_{4}$; the optical density was measured at $450 \mathrm{~nm}$.

Clones giving positive signals and with a unique BstNI fingerprint (New England Biolabs, Beverly, Mass.) were analyzed by sequencing with the $T 7$ sequencing kit (Pharmacia) with the M13rev primer, the genelII primer, and two primers located in the linker (LINKfwd and LINKrev) (Table 1). Plasmid DNA was purified with the QIAGEN kit (Qiagen).

\subsection{Characterization of scFvs}

For the preparation of periplasmic fractions by the borate-buffered saline shock procedure (58), clones obtained by transfection to the nonsuppressor strain TOP F' were cultured on 50-ml scale and induced as described previously (43). The fractions were analyzed on 15\% polyacrylamide gels and then blotted onto nitrocellulose. After blocking with a skim milk solution ( $5 \%$ [wt/vol] in Tris-buffered saline [TBS]), the scFv was detected with anti-FLAG M2 diluted $1 / 4,000$ in TBS- $0.05 \%$ Tween (TBST). After a $2-\mathrm{h}$ incubation period, the anti-FLAG was detected with anti-mouse Ab-horseradish peroxidase and the ECL detection kit (Amersham, Buckinghamshire, United Kingdom).

For the purification of $\mathrm{scFv}$, relolding was used in combination with affinity chromatography on 24 columns. The pelleted cells from a $50-\mathrm{ml}$ culture were resuspended in 8 $\mathrm{ml}$ of an $8 \mathrm{M}$ urea solution (in PBS) and sonicated. The mixture was rotated head-over-head for $30 \mathrm{~min}$, and insoluble material was removed (centrifugation for $30 \mathrm{~min}$ at $13,000 \mathrm{xg}$ ). The supernatant was dialyzed against PBS with four buffer changes. Insoluble proteins were removed by centrifugation, and after passing through a $0.2-\mu \mathrm{m}$-pore-size filter, the flowthrough was immediately loaded on a p 24 column (bed volume, $0.3 \mathrm{ml}$ ). The column material was prepared by coupling $8.4 \mathrm{mg}$ of protein to $1 \mathrm{~g}$ Tresyl sepharose in accordance with the supplier's instructions (Pierce). Washing and elution were performed as in the panning procedure. The yield was determined by measuring the optical density at $280 \mathrm{~nm}$, assuming that an $\mathrm{scFv}$ has a molar extinction coefficient $\left(\mathrm{E}^{1 \%}{ }_{280 \mathrm{~nm})}\right.$ of $14.3(27)$.

In order to establish the valencies of the scFvs, the molecular weight of the antigen binding molecules were determined by gel filtration (27) on a Superdex 75HR column (Pharmacia), which was calibrated with a mixture of BSA, chymotrypsin, ovalbumin, and RNase A (Pharmacia). As a control, an anti-human chorionic gonadotropin (anti-hCG) scFv selected from a murine immune library was used (unpublished data). Either $20 \%$ of a periplasmic fraction from 
a $50-\mathrm{ml}$ culture or affinity-purified scFv was injected onto a calibrated column. Fractions $(0.5$ ml) were collected and analyzed on Western blots.

Epitope mapping was performed with the pepscan method (22). Overlapping dodecapeptides of p24 from HIV strain ANT70, coupled to the solid support and obtained from the Department of Molecular Recognition of the Institute for Animal Science and Health (IDO$\mathrm{DLO})$, were tested with the scFv. The periplasmic fraction of clone D2 was diluted 100-fold in super Q buffer (PBS containing 5\% [vol/vol] horse serum, 5\% [wt/vol] BSA and $1 \%$ [vol/vol] Tween 20) to a concentration of $80 \mathrm{ng} / \mathrm{ml}$. After $16 \mathrm{~h}$ of incubation at $4^{\circ} \mathrm{C}, \mathrm{scFv}$ bound to peptides on the solid support was detected as described above.

\subsection{Affinity measurements in solution}

The affinities measured by the "in solution" method were determined with surface plasmon resonance on BIAcore (Biacore AB, Uppsala. Sweden) in accordance with the suggested procedures described in the BIAevaluation software. A high-density p24-coated (3777 Response Units [RUs] of immobilized Ag) CM-chip (Biacore $A B$ ) was prepared by covalent coupling according to the supplier's recommendations. A fixed amount of scFv or MAb, yielding a signal of between 1,000 and 1,500 RUs when injected onto the p24 chip, was mixed with a variable amount of $\mathrm{Ag}$ in a volume of $60 \mu \mathrm{l}$ by using HEPES- buffered saline (10 mM HEPES, $3.4 \mathrm{mM}$ EDTA, $150 \mathrm{mM} \mathrm{NaCl}, 0.05 \%$ surfactant $\mathrm{P} 20$ [pH 7.4]) as dilution buffer. After a 1-h period, needed to achieve equilibrium, the samples were injected. For the quantification of frec $A B$, a standard curve was prepared by injection of a dilution series of the tested $A b$ on the same flow cell (37).

\subsection{Nucleotide sequence accession numbers}

The GenBank accession numbers for the sequences of the antibodies reported in this study are as follows: D2 $V_{H}, A F 083186 ; A 2 V_{H}, A F 081541$; A3 $V_{H}, A F 081545$; A5 $V_{H}, A F 081543 ; D 1 V_{H}$, AF083189; D2 $V_{L}, A F 083188 ; A 2 V_{L}, A F 081542 ; A 3 V_{L}, A F 083185 ; A 5 V_{L}, A F 081544, D 1$ $\mathrm{V}_{\mathrm{L}}, \mathrm{AF} 083187$.

\section{Results}

\subsection{Library construction and selections}

Our major aim was to evaluate the library technology as a substitute for hybriduma technology for the generation of anti-HIV-1 Abs, which might be uscful for in vitro diagnostics. Theretore, we immunized a mouse with a lysate prepared from HIV-l inlectid H9 cells (52). After amplification of the V-genes of the murine spleen B cells and cloning in the phagemid vector pVI, a library containing $10^{7}$ clones was obtained. Seventy-five percent of the clones 
expressed scFvs, as was determined by PCR screening and by inspecting the morphology of the colonies: clones which express gene III (fusions) are usually visible as flat colonies, while those having an interrupted frame exhibited a more solid appearance (14a).

The library was subjected to four rounds of panning on recombinant p24. Four rounds of panning produced a 100 -fold enrichment in the number of eluted phages, indicating the presence of Ag-specific phage Abs. The scFvs produced by 48 individual clones after round 4 were analyzed by ELISA (43). Seventy percent of the tested scFvs produced high signals on viral lysate and p24 Ag, whereas no responses were found on BSA. After 24 clones were DNA fingerprinted by $B$ st $N 1$ digestion (43), two different pattems were identified; five clones representing the two patterns were selected for sequence analysis and further binding analysis. All five clones analyzed had minor differences in sequences and were classified as scFv D2 (Fig. 1).

Immune libraries usually contain a very diverse set of Ag-specific Abs $(14,44)$, thus, we modified our method of selection to retrieve phage Abs other than the ones found before. Selections were repeated with soluble (biotinylated) recombinant p24 (Sol-p24) and with p24coated magnetic particles (Bead-p24). After four rounds, enrichment factors of 100 and 10,000 were found for Sol-p24 and Bead-p24, respectively. Concomitant with this enrichment in the absolute number of eluted phages, we found an enrichment in the fraction of clones producing scFv, as determined by colony morphology. The percentage of scFv-producing clones increased from 50 to $75 \%$ of the clones before selection and after round 1 to more than $99 \%$ after round 4 . At this stage, 8 of 11 clones of Sol-p24 and 12 of 12 of Bead-p24 contained a complete insert, whereas Bst $\mathrm{N} 1$ fingerprinting revealed four different but closely related clones for Sol-p24 and only one type for Bead-p24. The four Sol-p24 scFv clones reacted positively on p24 and negatively on BSA, while the Bead-p24 clones gave high-level responses on p24 and BSA. Obviously, panning with the beads resulted in the isolation of an scFv specific for the blocking reagent $\mathrm{BSA}$, which was also used for blocking of the microtiter plates, which explains the positive signals in the p24 ELISA. The selection of BSA-specific clones can be explained by the presence of this $\mathrm{Ag}$ in the viral lysate used for immunization and its use for blocking the beads and the microtiter wells. We chose three different p24 binders of Sol-p24 and the Bead-p24 BSA binder for sequencing and further characterization. For Sol-p24, the clones designated A2, A3 and A5 were used: for Bead-p24, the clone was named D1.

Figure 1 (on next page). Deduced amino acid sequences of the variable heavy and light chain regions and germline origin of the p24 Abs derived from the murine library. Identical residues are indicated by dashes, while different residues are shown with uppercase characters and primer-encoded differences are indicated by lowercase characters. 


\section{A. Heavy chains}

Antigan Clone

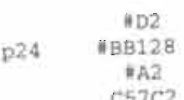

p24

IA3
WA5
C5?

C57/C2?

BSA

C57C2
CDRI

FR2

$\operatorname{CDR} 2$

FR1 CDRI FR2
OVQLQOSGPDLVKPGASVKISCKASGYSFT GYYMH WVRQSHGKSLEWIG RVNPNNGGTSYNQKFKG KATLTVDKSSNTAYMELRSLTSEDSAVYYCAT AT

$\mathrm{CDR}^{3}$

FR4

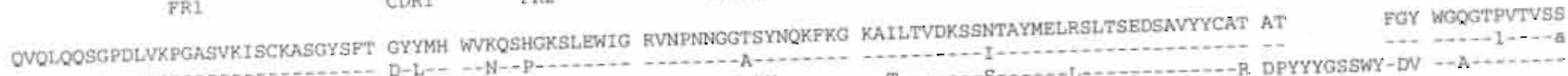
OVQLQOSGPDLVKPGASUKISCKASGTSPT QR e

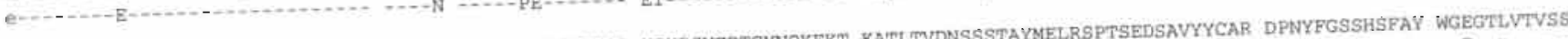

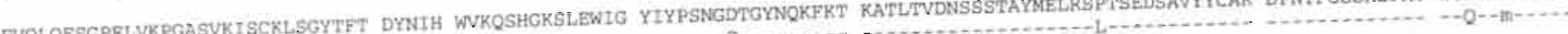

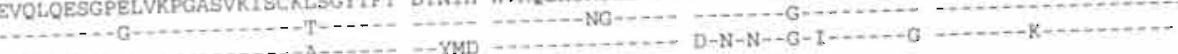

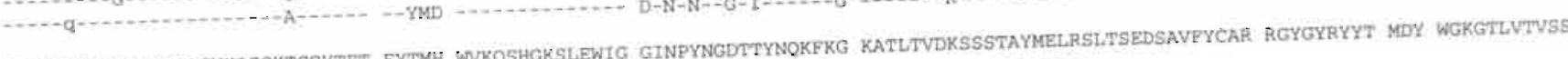
QVQLQQSGPELVKPGASVKISCKTSGYTPT EYTMH WVRQSHGKSLEWIG GINPYNGDT-S

\section{B. Light chains}

Antigen clone

DIOMTQSPASLSVSVGETVTITC RASENIY

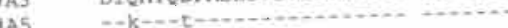

SNTA WYOKOCKSPQLLYY NAKTSAE GVPRFSGSGSTQFSLKINSPOPEDPGSYYC QHHYGTPFT FGSGTYLELKF

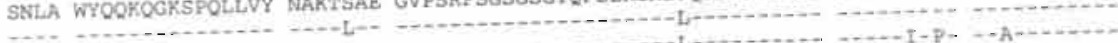
Y.. W.........

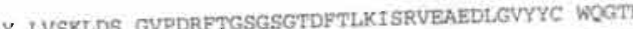




\subsection{Characterization of isolated scFvs}

Figure 1 shows the deduced amino acid sequences of the $V_{H}$ and $V_{L}$ gene products of the clones derived from panning the murine library with Ag coated polystyrene plates (D2) and with biotinylated $\mathrm{Ag}$ (A2, A3 and A5) together with the most related mouse germline analogues (V regions). The sequence of the BSA binding antibody (D1) selected with the beads is also included.

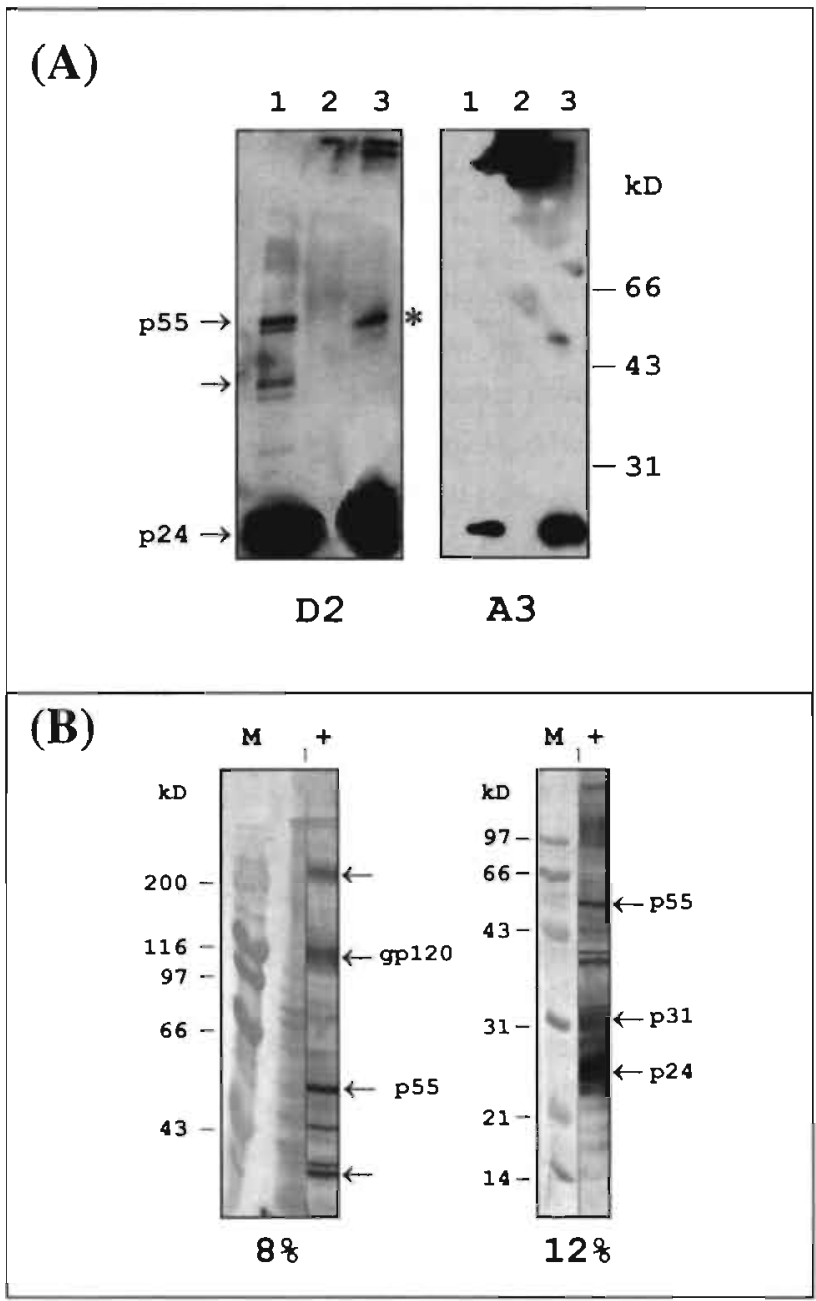

Figure 2. Western blot detection of p24 by scFvs and mouse polyclonal Abs.

(A) Detection with scFv D2 (left panel) and A3 (right panel). Viral lysate (lanes 1), BSA as a negative control (lanes 2), and purified recombinant p24 (lanes 3) were analyzed on a $12 \%$ gel. The band indicated with an asterisk represents the dimer of p24. present in the recombinant and in the viral product.

(B) Blot analysis of viral lysate with polyclonal serum (strips labeled with +) obtained from the mouse used for library construction (left blot made with an $8 \%$ gel and the right one with a $12 \%$ gel). Amido black-stained markers (lanes labeled $\mathrm{M}$ ) are shown. The Gag precursor p55 and its derivative p24, the envelope protein $\mathrm{gp} 120$, and the polymerase $\mathrm{p} 31$ are indicated with arrows. The slow-migrating protein indicated with the top-unlabeled arrow in the left blot is probably the envelope precursor gp 160 . The other protein marked with the bottom-unlabeled arrow, is a cleavage product of Gag, which is also recognized by scFv D2 (Fig. 2A).

The four anti-p24 scFvs use highly homologous $\mathrm{V}_{\mathrm{H}}$ gene segments. They belong to class IIA according to Kabat's classification (36) and use two germline gene segments only. Clones $\mathrm{A} 2$ and $\mathrm{D} 2$ and clones $\mathrm{A} 3$ and $\mathrm{A} 5$ can be grouped in two classes depending on the germline used. D2 has a divergent and shorter CDR3 than clone $A 2$ and thus is clonally not related. The 
light chain of clone D2 could be matched adequately with a germline segment, and is very different from the A2 light chain. The kappa light chains of the three Abs A2, A3 and A5 use a nearly identical $V_{\kappa}$ gene segment, derived from the germline segment coded k2/MMIG27 (for classification and the codes used in Fig. 1, see "Germline gene directories of the mouse" homepage (http://www.ibt.unam.mx/ almagro/V_mice.html)).

The specificities of the scFis were determined by Western blot analysis (Fig. 2). Periplasmic fractions containing s $\mathrm{F} F v$ were used for the detection of viral and recombinant p24. Two different recognition patterns were obtained. Abs A2, A3, and A5 reacted with (the reduced form of) $\mathrm{p} 24$, in a viral lysate, and as a recombinant product (for example, the blot incubated with scFv A3 is shown in Fig. 2A). ScFv D2 also recognizes p24, but it also detects the viral Gag polyprotein p55 (Fig. 2A, left panel, lane 1) from which the matrix protein p17, the nucleocapsid p7, and the capsid protein p24 are proteolytically processed. Another product with a molecular weight of approximately $40 \mathrm{kDa}$ is visible. This protein seems also to be recognized by polyclonal serum (Fig. 2B) obtained from the mouse that was used for the construction of the library. The $\mathrm{p} 24$ dimer, which is the predominant form of the native protein as was observed by gel filtration (results not shown), might also be detected with mouse serum (Fig. 2B, left panel, band between p55 and the $40-\mathrm{kDa}$ derivative). Indeed, the native p24 protein can form a complex of oligomers in solution, including dimers, tetramers, dodecamers, spheres, fibers and tubes (18). An intense reactivity of the original murine polyclonal serum against p24 and its precursors can be observed, while the mouse seems to have a lower Ab titer against the envelope protein gp 120 . Thus, the original library was biased in its immune response towards $\mathrm{p} 24$, which is probably caused by the abundance of the antigen in the viral lysate used for immunization ( 10 , 63).

\subsection{D2 is a Diabody}

After performing the sequence analysis of clone D2, selected on immobilized p24, we noticed that it contained a linker consisting of 2 amino acids (GlySer) rather than 15 amino acids $\left(\left(\mathrm{Gly}_{4} \mathrm{Ser}\right)_{3}\right)$. As suggested in the literature (29), shortened linkers may lead to the formation of dimeric molecules with two functional binding sites, the so-called 'diabodies'. The shorter linker in clone D2 might therefore be responsible for the formation of a bivalent scFv molecule, and this type of molecule might be strongly enriched over the other anti-p24 scFvs by its greater avidity advantage in the panning selection. We generated a number of D2 derivatives and characterized their behavior.

From a light chain shuffling library, we selected a derivative of clone D2, that had an identical light chain and a complete 15-amino-acid linker; the clone was designated D2/15. We examined the molecular weight of the antibody fragments present in the periplasmic space of the producing bacteria by gel filtration on a calibrated Superdex $75 \mathrm{HR}$ column (27): the obtained 
chromatogram of clone D2 is shown in Fig. 3A. The scFv with a two-amino-acid linker emerged from the column as a single peak with a molecular weight of $45 \mathrm{kDa}$. as could be deduced from Western blot analysis with the fractions collected during gel filtration (Fig. 3B). Both scFvs, $\mathrm{D} 2 / 15$ and a control anti-hCG Ab fragment with a 15-residue linker, gave two peaks: the dimeric fraction at $45 \mathrm{kDa}$ and a monomeric fraction at $25 \mathrm{kDa}$.

By chain shuffling the light chain of the D2 diabody, clone D2/15 was isolated by using an excess of $\mathrm{Ag}$ during selection. By increasing the stringency of selection, i.e. decreasing the $\mathrm{Ag}$ concentration from $100 \mathrm{nM}$ to $100 \mathrm{pM}$, the monomeric clone D2/15 was completely lost and the selected population was overtaken by (avid) diabodies. We retrieved no D2 derivatives with other light chains, not even the one of clone $A 2$, in spite of its highly related $V_{H}$ gene, as was also reported by others (56). However, it should be emphasized that the diabody's CDR3 sequence is completely different from clone $\mathrm{A} 2$ in length as well as in sequence. Due to the close proximity of $\mathrm{H} 3$ to the light chain variable-region loops, this may determine the pairing light

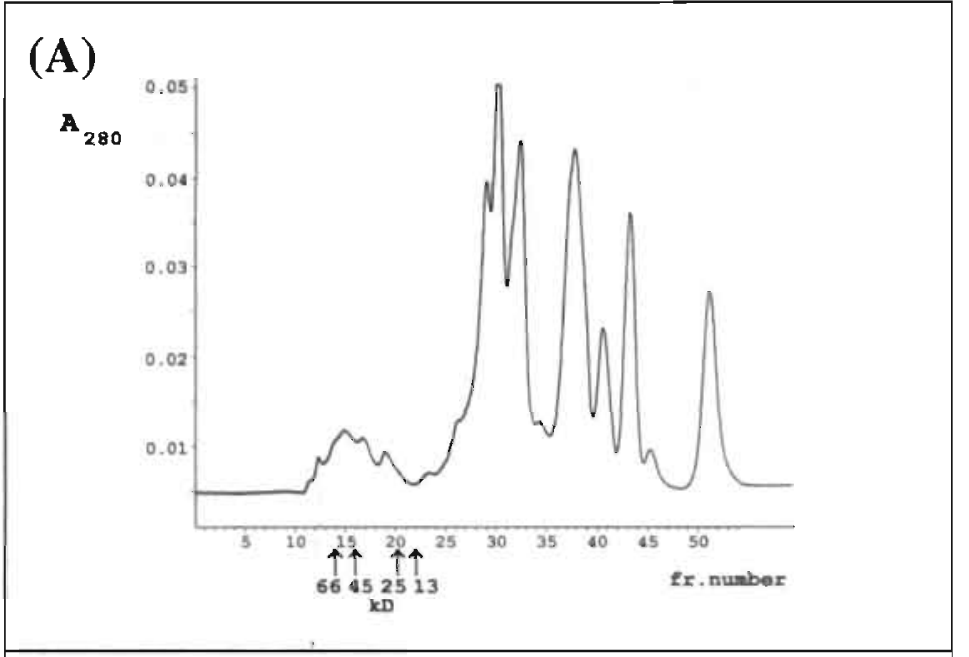

\section{(B)}

Eraction: 1314151718192113141517181920211314151718192021

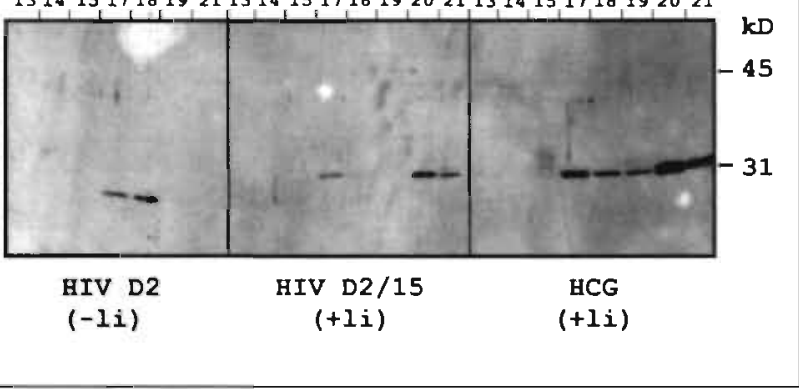

Figure 3. Gel filtration of periplasmic fractions on Superdex $75 \mathrm{HR}$ and analysis of the collected fractions on Western blot with anti-FL.AG M2.

(A) Chromatogram, obtained by plotting $A_{280}$ against the fraction number. illustrating the separation of the periplasmic proteins from clone D2. The retention times of the marker proteins are indicated with arrows.

(B) Western blot analysis ( $15 \%$ gel) of the collected fractions (fraction number is shown above the blots) obtained from gel filtration of anti-p24 clones and of an anti-hCG scFv-producing clone. Two peak fractions can be discriminated in the scFvs having a complete linker (the derivative D2/lS and the anti-hCG): a main peak of the monomer (fraction 20; molecular mass $25 \mathrm{kDa}$ ) and a dimer peak (fraction 17; molecular mass $45 \mathrm{kDa}$ ). ScFv of clone D2 emerged from the column in a single peak corresponding to the dimeric product. 
chain. In the course of the shuffling experiment, we isolated one clone, D2/L3mut, which appeared to have a 15-residue linker but still produced predominantly dimeric scFvs. Sequencing revealed a single mutation from the D2 sequence, of residue W (shown in boldface type in Fig. 1) to $Y$, in the third hypervariable region of the light chain. Since this region of the $\mathrm{Ab}$ is in close contact with the $\mathrm{V}_{\mathrm{H}}$ region, the mutation may destabilize the $\mathrm{V}_{\mathrm{H}}-\mathrm{V}_{\mathrm{L}}$ association, thereby promoting dissociation and intermolecular pairing (56).

\subsection{Affinity measurements}

For affinity measurement, the scFvs were purified by affinity chromatography on $\mathrm{Ag}$ columns. The scFv fragments were refolded after denaturation of all cell proteins with urea by subsequent dialysis against PBS. Although the efficiency of refolding is limited, the yield of functional scFv is much higher than in periplasmic fractions obtained by the osmotic shock procedure (58). The purity was analyzed on a Coomassie blue-stained gel (data not shown).

All purified scFvs were analyzed by gel filtration chromatography, as was already performed with the periplasmic fraction of clone D2 (Fig. 3); the purified product of the D2 diabody contains dimers only, while the analogue with the complete linker, D2/15, is

Table 2. Affinities of the anti-p24 MAbs and scFvs

\begin{tabular}{ccccc}
\hline Ab type & Ab & form $^{\mathbf{a}}$ & ${\text { linker length }(\mathbf{a a})^{\mathbf{b}}}$ & $\mathbf{K}_{\mathbf{d}}(\mathbf{M})$ \\
\hline $\mathrm{MAb}$ & $5 \mathrm{~B} 4$ & & & $3.6 \times 10^{-8}$ \\
& $34 \mathrm{~A}$ & & & $8.0 \times 10^{-9}$ \\
& $39 \mathrm{~B}$ & & 15 & $8.0 \times 10^{-9}$ \\
& & & & \\
$\mathrm{ScFv}$ & $\mathrm{A} 2$ & nd & & $2.0 \times 10^{-6}$ \\
& $\mathrm{~A} 3$ & monomer & 15 & $2.5 \times 10^{-8}$ \\
& $\mathrm{~A} 5$ & monomer & 15 & $3.0 \times 10^{-8}$ \\
& $\mathrm{D} 2$ & dimer & 2 & $1.0 \times 10^{-6}$ \\
& $\mathrm{D} 2 / 15$ & monomer & 15 & $3.0 \times 10^{-6}$ \\
& $\mathrm{D} 2 \mathrm{~L} 3 \mathrm{mut}{ }^{\mathrm{d}}$ & dimer & 15 & $1.3 \times 10^{-6}$ \\
\hline
\end{tabular}

"As determined by gel filtration.

aa, amino acids.

ND, not determined.

This clone is identical to clone D2 but with a complete linker and a single mutation within CDR3 of $\mathrm{V}_{\mathrm{K}}$ (see text). 
predominantly monomeric. Clones A3 and A5 produce monomeric seFv fragments only (Table 2).

We compared the affinities of the library-derived scFvs with the binding strength of hybridoma-produced MAbs. We chose to measure the kinetics of binding by surface plasmon resonance on a BIAcore machine. When using $\mathrm{Ag}$ immobilized on sensorchips, we always observed biphasic dissociations, probably caused by the oligomerization of p24 (18), resulting in a heterogeneous coat. The abnormal sensorgrams were also noted before by others (25). Therefore, the affinity was determined in solution (47), by injecting a preestablished equilibrium mixture of a fixed amount of $\mathrm{Ab}$ and a variable amount of $\mathrm{Ag}$ on a sensorchip with a highdensity coat of $\mathrm{Ag}$. Under mass transfer limiting conditions, the amount of free $\mathrm{Ab}\left(\mathrm{Ab} \mathrm{b}_{\text {free }}\right)$ is proportional to the binding rate. Subsequently the $K_{t}$ is obtained by plotting the concentration of $A b_{\text {free }}$ (or the fraction $A b_{\text {free }} / A b_{0} ; A b_{0}$ is the concentration $A b$ when no $A g$ has been added) against the total concentration of $\mathrm{Ag}$. In Fig. 4, the binding curves derived from the MAbs and scFvs are shown; Table 2 gives the corresponding $K_{d} \mathrm{~s}$. The affinities of the MAbs 34A and 39B are threefold higher than those of scFvs $\mathrm{A} 3$ and $\mathrm{A} 5$, both selected on biotinylated Ag, which in turn have slightly higher affinities than MAb 5B4. The D2 diabody, selected on immobilized p24 via panning, has a much lower affinity (micromolar range). The monomeric version of D2, D2/15, has a threefold-lower affinity than that of its dimeric counterpart, demonstrating that the effect of avidity on the apparent affinity determined in this assay is limited. Finally, clone D2/L3mut, which expressed mainly dimeric scFv (as gel filtration of affinity-purified scFv revealed [results not shown]) had an affinity approaching that of clone D2.

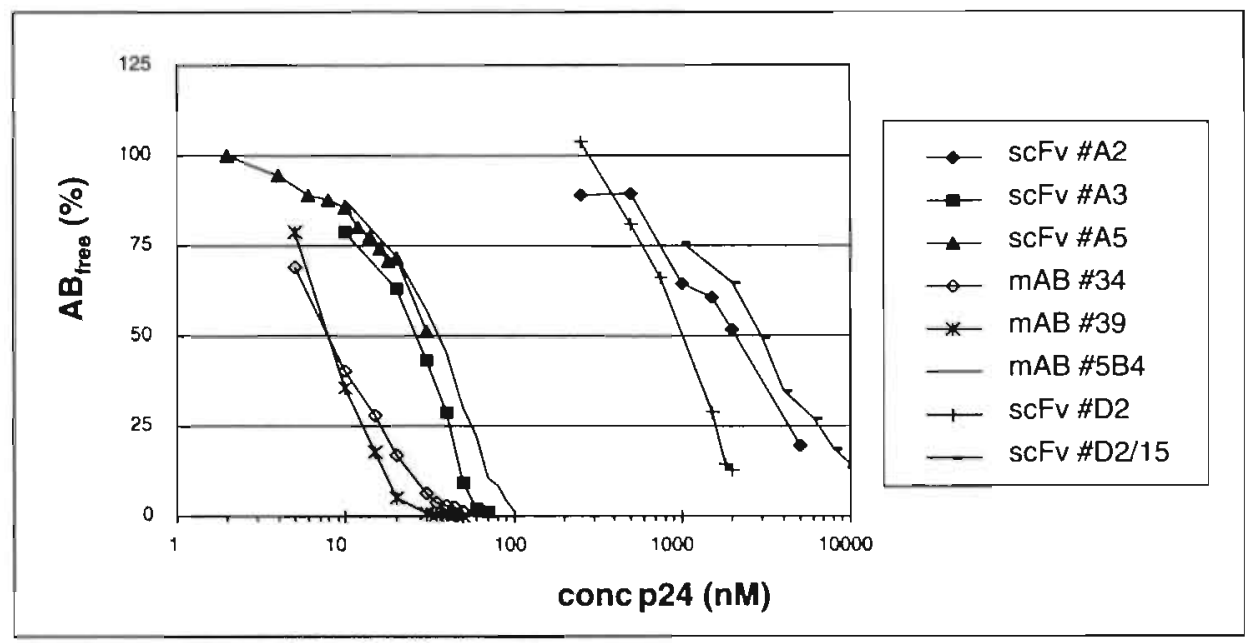

Figure 4. The percentage of $\mathrm{Ab}_{\text {free }}$ as a function of $\mathrm{Ag}$ concentration (logarithmic scale). The amount of $A b_{\text {free }}$ (i.e., not bound to soluble $\mathrm{Ag}$ ) was determined by surface plasmon resonance and related to the input amount $\mathrm{Ab}$ (no $\mathrm{Ag}$ added). The $K_{d}$ was derived from the plots at $50 \%$ binding. 


\subsection{Epitope recognition}

Pepscan analysis (22) was used in the identification of the epitopes of the scFvs. Although all recombinant fragments recognized denatured $\mathrm{Ag}$ on Western blot, only the diabody type D2 gave a good response. The results obtained with the overlapping dodecapeptides based on the

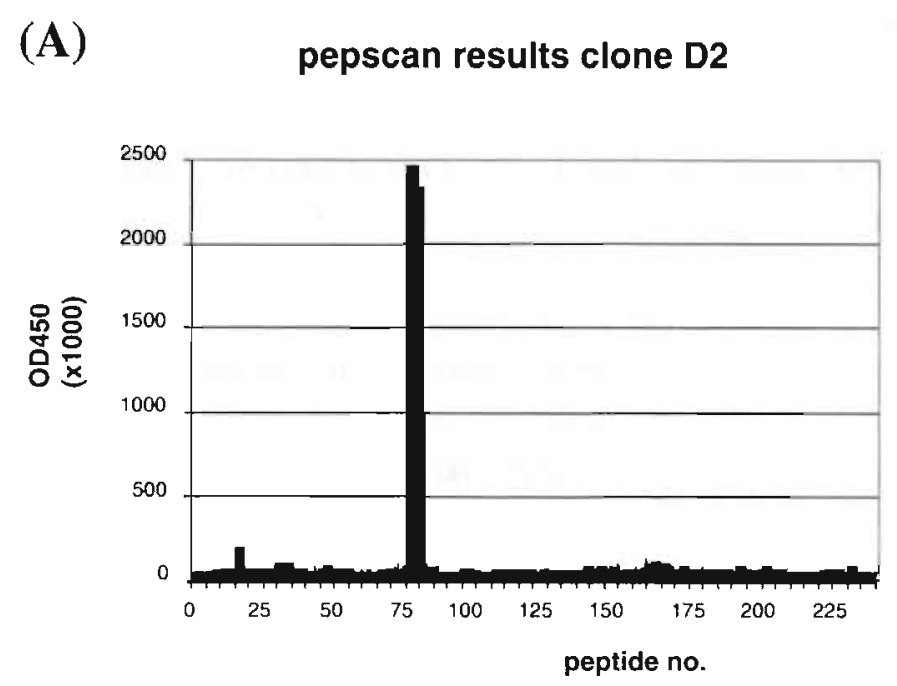

\section{(B) \\ pepscan with HIV-1 patient serum}

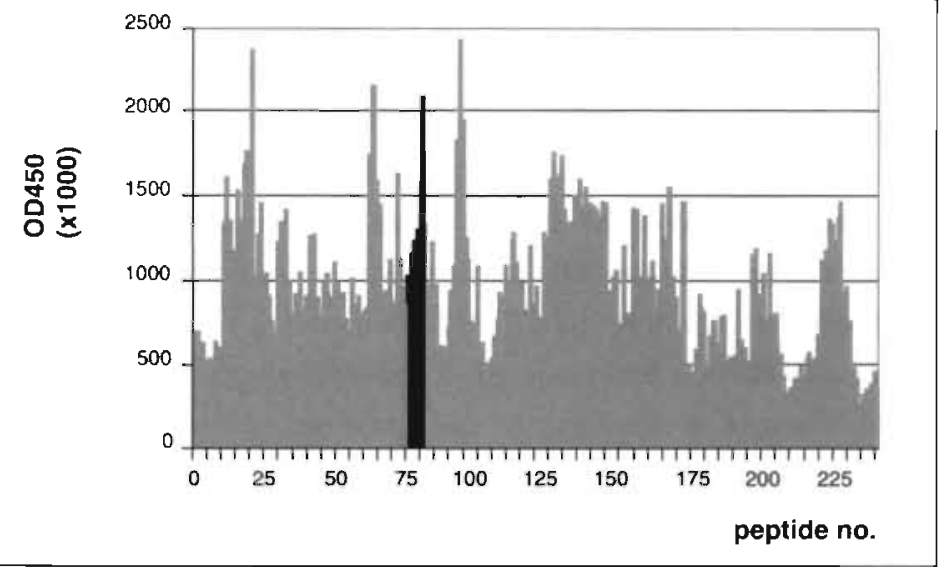

Figure 5. Pepscan results obtained with dedecapeptides of 224 of HIV-1 strain ANT70 with scFv D2 (A) and serum Abs of an HIV-1 patient (B).

The numbering on the horizontal axis corresponds with the amino terminal amino acid of the peptide; the first peptide starts with a $Q$ at -3 relative to the cleavage site of p17 and p24, the last peptide (no. 240) ends with $A$ located at -3 relative to the cleavage site of p2 with p7. On the vertical axis, the optical density at $450 \mathrm{~nm}$ obtained for each peptide in ELISA has been plotted. The common sequence recognized by the human serum and scFv D2, starting with peptide 76 and ending with peptide 82 , is indicated in panel $\mathbf{B}$ by solid bars. 
sequence of HIV-1 subtype O strain ANT70 and the scFv-containing periplasmic fraction are shown in Fig. 5A. The $A b$ reacted with the peptides starting with the sequence INEEAVEEWDRTH and ending with EWDRTHPPPVGP, thereby having in common the sequence EWDRTH. This sequence is also recognised by serum Abs from an HIV-1 patient (Fig. $5 \mathrm{~B}$ ), indicating that the immune system of the mouse recognizes the same epitope on the immunized $\mathrm{Ag}$ as that of humans after virus infection. It should be noted that the p24 used for immunization and selection is derived from strain IIIB, while the pepscan is based on strain ANT70. For the A2, A3, and A5 Abs, the pepscan based on the ANT70 and the IIIB sequences did not reveal any unique epitopes (results not shown), indicating that these Abs may recognize a conformation-sensitive epitope.

Inspection of the HIV-1 B-cell epitope database (Los Alamos) showed us that several MAbs that share the epitope with scFv \#D2 have been found. From one of these, hybridoma BB128y (61), the $\mathrm{V}$ gene sequence contains a nearly identical heavy chain variable region, with a remarkably short $\mathrm{H} 3$ loop, and, even more surprisingly, a light chain variable region with only one residue difference in the germline-encoded region from D2 (Fig. 1).

\section{Discussion}

\subsection{Isolation of different sets of anti-p24 Abs dependent on the selection procedure}

When we used the combinatorial library technology for the generation of MAbs, we found that the selection method determines to a great extent what type of $\mathrm{Ab}$ will be found. By using Ag-coated polystyrene, the high Ag density probably favors the selection of scFvs which are avid, exemplified by the selection of the D2 clone, while selection with soluble Ag leads to the isolation of monomeric $\mathrm{Ab}$ fragments produced by clones $\mathrm{A} 2, \mathrm{~A} 3$, and $\mathrm{A} 5$. In the course of the assembly reaction between $V_{H}$, linker, and $V_{L}$ to generate scFvs with 15-residue linkers (14), mispriming may infrequently lead to short-linker versions of $\mathrm{V}_{\mathrm{H}}$ and $\mathrm{V}_{\mathrm{L}}$ as reported previously (42). In the resulting Abs with a 5- or 10-residue linker, structural constraints prevent the intramolecular pairing of $\mathrm{V}_{\mathrm{H}}$ and $\mathrm{V}_{\mathrm{L}}$. Instead, the product forms an intermolecular pair with another $\mathrm{V}_{\mathrm{H}}-\mathrm{V}_{\mathrm{L}}$ fusion product, yielding a bivalent $\mathrm{scFv}$ molecule, the so-called diabody $(29,44)$. The D2 diabody is heavily dependent on avidity for its selection; when a selection procedure with soluble p24 is used, the avidity effect is less pronounced and other clones (A3 and A5) are selected on the basis of affinity. The avidity effect may be more pronounced also by the intrinsic aggregation behavior of $\mathrm{p} 24$, which upon coating may lead to epitopes that are spatially ordered such that they are more prone to cross-linking by scFv Abs. Alternatively, the epitope recognized by clones $\mathrm{A} 3$ and $\mathrm{A} 5$ might be shielded or modified on $\mathrm{p} 24$ adsorbed onto polystyrene, although this hypothesis was not supported by the high ELISA signals. Our data indicate that selections should be carefully fine-tuned to retrieve the desired Abs. To obtain a wider panel of anti-p24 
Abs, the various selection procedures used here may be altered or extended, for example, by using epitope-shielding Abs as described elsewhere (16).

The affinities of the Abs were determined with an in solution method. The multimerization behavior of p24 complicated the affinity determination by direct kinetic measurement, while the alternative assay gave reliable data with only a minor effect of avidity. The affinities of the monomeric scFvs $\mathrm{A} 3$ and $\mathrm{A} 5$ found by selection with soluble $\mathrm{Ag}$ are in the nanomolar range and are slightly better than the affinity of one of the studied MAbs, which was taken from a panel of hybridoma-produced MAbs that are currently used in diagnostic assays. Their affinity is still threefold lower than of the best MAbs tested, but this might be due to the avidity of the MAbs. Indeed, a threefold difference was also found between the dimeric and the monomeric versions of clone D2.

The present study showed that $\mathrm{Ab}$ fragments obtained from the murine library have comparable affinities to those of their hybridoma-derived analogues. For obtaining high-affinity Abs useful as diagnostic reagents, avidity may be recruited, for example, by making diabodies (29), scFv trimers (39), or tetramers (for a review, see reference 51). The affinity of the best selected antibodies, A3 and A5, may be improved, for example, by targeted mutagenesis (1, 30, 57). Finally, additional alternative selections should be employed to further explore the diversity of the murine phage library that was constructed.

\subsection{D2 recognizes an immunodominant epitope closely located at the CypA binding site}

All selected scFvs recognized linear epitopes of p24, as was concluded from Western blot studies. p24 expressed in E. coli as well as Ag, derived from cultured virus and presented in a reduced form was reactive on Western blot. Pepscan analysis with overlapping dodecapeptides yielded the epitope of the scFv produced by clone D2. The three-dimensional structure of the amino-terminal core domain of the HIV-1 capsid protein is known (24); it reveals the presence of an arrowhead-shaped domain, with seven helices and two $\beta$ hairpins, and an exposed partially ordered loop involved in binding cyclophilin A. Recently, the binding domain was analyzed in more detail by crystallization of a peptide fragment of p24 with cyclophilin A (69), indicating the direct interaction of residues Ala 88, Gly 89 , Pro 90 , and Ile 91 with residues from the cellular enzyme. The epitope of D2 is located within the carboxy-terminal part of helix IV, ending at His 84 (or Leu in strain $\mathrm{HIB}$ ), and it is separated by three residues from the residues interacting with cyclophilin A.

The interaction of p24 with cyclophilin A appears to result in the packaging of approximately 200 copies of CypA into each HIV-1 virion. Although the function of CypA remains unclear, virions lacking the enzyme are known to be poorly infectious (7). Cyclosporins capable of binding to cyclophilin $A$ are effective in inhibiting virus replication, as was reported before $(4,41,60)$. Agents that interfere with the CypA binding function of p24 may be useful in reducing viral load; application of our scFv D2 as intracellularly expressed Ab may therefore 
prevent binding of the enzyme in infected cells, rendering the progeny virus less infectious or noninfectious, as was done successfully with anti-Tat (45) and anti-gpl20 (12) Abs.

\subsection{Retained $V_{H}-V_{L}$ pairing}

Several epitopes have been mapped within the CypA loop region, which by its exposed orientation is accessible for reaction with Abs. Other MAbs that recognize the same epitope have been reported $(28,34,48,49,50,54,55)$. In addition, the structure of a complex between an anti-p24 Ab and its $\mathrm{Ag}$ was determined with an $\mathrm{Ab}$ with nearly identical epitope recognition as Ab D2 (46). Morcover, the epitope is also reactive with human Abs, as indicated by our pepscan with serum from an HIV-1-infected patient and as was also concluded in a study in which the peptide was incorporated in a diagnostic assay (35).

Despite the recognition of the same epitope by the Abs mentioned above, their heavy chain $\mathrm{V}$ genes have little homology to each other, except that they both use a CDR3 of the same size and a nearly identical CDR1. The most striking resemblance exists between scFv D2 and antip24 MAb BB 128 $\gamma(61)$, which have almost the identical $V_{H}$ and $V_{K}$ sequence (differences of six and two amino acid residues, respectively). Clones isolated from a $V_{\kappa}$ light chain shuffling library were found to have the original MAb light chain sequence, including the tyrosin (replacing the tryptophan present in clone D2), indicating a favorable selection for original pairings. It has been argued that the chances of retrieving the original pairing from a combinatorial library are very low. as the result of RNA isolation (67), and that it is particularly difficult to retrieve the original light chain (2). During the early days of combinatorial library construction with the bacteriophage lambda system, an original $\mathrm{V}_{\mathrm{H}}-\mathrm{V}_{\mathrm{L}}$ combination identical to the one found in a hybridoma $\mathrm{Ab}$ was reported (11). However, the identity was less pronounced than that with our p24 scFv: in the partially sequenced $V_{H}$, containing CDR2, FR3, and a part of FR2, there were eight mismatches present and the light chain comprised four differences. Moreover, the $\mathrm{V}_{\mathrm{H}}$ could pair with a different $\mathrm{V}_{\mathrm{L}}$, as was also found by Barbas and colleagues (2). Considering the results from the light chain shuffling library, the $D 2 V_{H}$ is strictly monogamous. i.e., it can pair with its original light chain only. When a mouse has $10^{\circ}$ different $B$ cells, a library with at least $10^{12}$ clones must be made to have a chance to identify an original $\mathrm{V}_{\mathrm{H}}-\mathrm{V}_{\mathrm{L}}$ combination. After immunisation, the number of anti-p24-expressing B lymphocytes will be larger, but still a rather huge library would be needed for finding original combinations. One of the reasons that we isolated the original clone may be because of a high titer of these Abs, since pepscan analysis with the polyclonal mouse serum gave a major response on the peptides containing the EWDRTH sequence (data not shown).

\subsection{Diagnostic applications}

The selected anti-p24 Abs could be used in a sensitive p24 Ag assay in which HIV-l infection could be detected in an early phase (20). The correlation between affinity and 
sensitivity in ELISA has been clearly demonstrated $(15,40)$. The affinities of the selected scFvs approach the values of MAbs, which can detect 5 to $10 \mathrm{pg} \mathrm{p} 24$ per $\mathrm{ml}$ of serum, when combined in a capture assay. An exception is scFv D2, with its micromolar level of affinity. It has been reported that the closely related $\mathrm{MAb} B \mathrm{~B} 128 \gamma$ is a synergistic $\mathrm{Ab}$, meaning that it its affinity is enhanced dramatically (up to 1.000-fold) when combined with MAbs, which recognize epitopes on the opposite side of the Ag. In vitro affinity maturation $(1,30,57)$ of the Abs might improve the sensitivity to a level comparable with that of PCR detection (6). The format of the Abs could be adapted to their application; for instance, a high-affinity monovalent fragment might be useful for capturing $\mathrm{Ag}$, while a multivalent scFv might be more suitable for the sensitive detection of high-density $\mathrm{Ag}$ arrested on a solid surface. Therefore, the application of engineered $\mathrm{Ab}$ fragments should be very useful for the development of new generations of diagnostics.

\section{References}

1) Barbas, C. F., and D. R. Burton. 1996. Selection and evolution of high-affinity human antiviral antibodies. Trends Biotechnol. 14:230-234.

2) Barbas, C. F., T. A. Collet, W. Amberg, P. Roben, J. M. Binley, D. Hoelstra, D. Cababa, T. M. Jones, A. Williamson, G. R. Pilkington, N. L. Haigwood, E. Cabezas. A. C. Satterhwait. I. Sanz, and D. R. Burton. 1993. Molecular profile of an antibody responsc to HIV-I as probed by combinatorial libraries. J. Mol. Biol. 230:812-823.

3) Baumberger, C., S. Kinloch-de-Loes, S. Yerly, B. Hirschel, and L. Perrin. 1993. High levels of circulating RNA in patients with symptomatic HIV-1 infection. AIDS 7 Suppl 2:S59. S64.

3a)Berman, M., unpublished.

4) Billich. A., F. Hammerschmid, P. Peichl. R. Wenger, G. Zenke, V. Quesniaux, and B. Rosenwirth. 1995. Mode of action of SDZ NIM 811, a nonimmunosuppressive cyclosporin $A$ analog with activity against human immunodeficiency virus (HIV) type 1: interference with HIV protein-cyclophilin A interactions. J. Virol. 69:2451-2461.

5) Boeke, J. D., P. Model, and N. D. Zinder. 1982. Effects of bacteriophage fl gene III protein on the host cell membrane. Mol. Gen. Genet. 186:185-192.

6) Boni, J., M. Opravil, Z. Tomasik, M. Rothen, L. Bisset. P. J. Grub, R. Luthy, and J. Schupbach. 1997. Simple monitoring of antiretroviral therapy with a signal-amplificationboosted HIV-1 p24 antigen assay with heat-denatured plasma. AIDS 11:F47-F52.

7) Braaten, D., E. K. Franke, and J. Luban. 1996. Cyclophilin A is required for an early step in the life cycle of human immunodeficiency virus type 1 before the initiation of reverse transcription. J. Virol. 70:3551-3560). 
8) Brookmeyer, R., and T. C. Quinn. 1995. Estimation of current human immunodeficiency virus incidence rates from a cross-sectional survey using early diagnostic tests. Am. J. Epidemiol. 141:166-172.

9) Busch, M. P., L. L. Lee, G. A. Satten, D. R. Henrard, H. Farzadegan, K. E. Nelson, S. Read, R. Y. Dodd, and L. R. Petersen. 1995. Time course of detection of viral and serologic markers preceding human immunodeficiency virus type 1 seroconversion: implications for screening of blood and tissue donors. Transfusion 35:91-97.

10) Casey, J. M., Y. Kim, P. R. Andersen, K. F. Watson, J. L. Fox, and S. G. Devare. 1985. Human T-cell lymphotropic virus type III: immunologic characterization and primary structure analysis of the major internal protein, p24. J. Virol. 55:417-423.

11) Caton, A. J., and H. Koprowski. 1990. Influenza virus hemagglutinin-specific antibodies isolated from a combinatorial expression library are closely related to the immune response of the donor. Proc. Natl. Acad. Sci. U.S.A. 87:6450-6454.

12) Chen, S. Y., Y. Khouri, J. Bagley, and W. A. Marasco. 1994. Combined intra- and extracellular immunization against human immunodeficiency virus type 1 infection with a human anti-gp120 antibody. Proc. Natl. Acad. Sci. U.S.A. 91:5932-5936.

13) Chirgwin, J. M., A. E. Przybyla, R. J. MacDonald, and W. J. Rutter. 1979. Isolation of biologically active ribonucleic acid from sources enriched in ribonuclease. Biochemistry 18:5294-5299.

14) Clackson, T., H. R. Hoogenboom, A. D. Griffiths, and G. Winter. 1991. Making antibody fragments using phage display libraries. Nature 352:624-628.

14a)De Haard, H. J. W., unpublished data.

15) Devey, M. E., and M. W. Steward. 1988. The role of antibody affinity in the performance of solid phase assays.. p. 135-153. In D. M. Kemeny, and S. J. Challacombe (ed.), ELISA and Other Solid Phase Immunoassays. John Wiley \& Sons Ltd, London.

16) Ditzel, H. J., J. M. Binley, J. P. Moore, J. Sodroski, N. Sullivan, L. S. Sawyer, R. M. Hendry, W. P. Yang, C. F. Barbas, and D. R. Burton. 1995. Neutralizing recombinant human antibodies to a conformational V2- and CD4-binding site-sensitive epitope of HIV-1 gp 120 isolated by using an epitope-masking procedure. J. Immunol. 154:893-906.

17) Dower, W. J., J. F. Miller, and C. W. Ragsdale. 1988. High efficiency transformation of $E$. coli by high voltage electroporation. Nucleic Acids Res. 16:6127-6145.

18) Ehrlich, L. S., B. E. Agresta, and C. A. Carter. 1992. Assembly of recombinant human immunodeficiency virus type 1 capsid protein in vitro. J. Virol. 66:4874-4883.

19) Fischl, M. A., D. D. Richman, N. Hansen, A. C. Collier, J. T. Carey, M. F. Para, W. D. Hardy, R. Dolin, W. G. Powderly, J. D. Allan, et. al. 1990. The safety and efficacy of zidovudine (AZT) in the treatment of subjects with mildly symptomatic human immunodeficiency virus type 1 (HIV) infection. A double-blind, placebo-controlled trial. The AIDS Clinical Trials Group. Ann. Intern. Med. 112:727-737. 
20) Center for Biologics Evaluation and Research 1995. Recommendations for donor screening with a licensed test for HIV-1 antigen. Food and Drug Administration, Rockville, M.D.

21) Foote, J., and H. N. Eisen. 1995. Kinetic and affinity limits on antibodies produced during immune responses. Proc. Natl. Acad. Sci. U.S.A. 92:1254-1256.

22) Geysen, H. M., R. H. Meloen, and S. J. Barteling. 1984. Use of peptide synthesis to probe viral antigens for epitopes to a resolution of a single amino acid. Proc. Natl. Acad. Sci. U.S.A. 81:3998-4002.

23) Gherardi, E., and C. Milstein. 1992. Original and artificial antibodies. Nature 357:201-202.

24) Gitti, R. K., B. M. Lee, J. Walker, M. F. Summers. S. Yoo, and W. I. Sundquist. 1996. Structure of the amino-terminal core domain of the HIV-1 capsid protein. Science 273:231235.

25) Glaser, R. W., and G. Hausdorf. 1996. Binding kinetics of an antibody against HIV p24 core protein measured with real-time biomolecular interaction analysis suggest a slow conformational change in antigen p24. J. Immunol. Methods 189:1-14.

26) Graziosi, C., G. Pantaleo, L. Butini, J. F. Demarest, M. S. Saag, G. M. Shaw, and A. S. Fauci. 1993. Kinetics of human immunodeficiency virus type 1 (HIV-1) DNA and RNA synthesis during primary HIV-1 infection. Proc. Natl. Acad. Sci. U.S.A. 90:6405-6409.

27) Griffiths, A. D., M. Malmqvist, J. D. Marks, J. M. Bye, M. J. Embleton, J. McCafferty, M. Baier, K. P. Holliger, B. D. Gorick, J. N. Hughes, H. R. Hoogenboom, and G. Winter. 1993. Human anti-self antibodies with high specificity from phage display libraries. EMBO J. 12:725-734.

28) Hinkula, J., J. Rosen, V. A. Sundqvist, T. Stigbrand, and B. Wahren. 1990. Epitope mapping of the HIV-1 gag region with monoclonal antibodies. Mol. Immunol. 27:395-403.

29) Holliger, P., T. Prospero, and G. Winter. 1993. "Diabodies": small bivalent and bispecific antibody fragments. Proc. Natl. Acad. Sci. U.S.A. 90:6444-6448.

30) Hoogenboom, H. R. 1997. Designing and optimizing library selection strategies for generating high-affinity antibodies. Trends Biotechnol. 15:62-70.

31) Hoogenboom, H. R., A. D. Griffiths, K. S. Johnson, D. J. Chiswell, P. Hudson, and G. Winter. 1991. Multi-subunit proteins on the surface of filamentous phage: methodologies for displaying antibody (Fab) heavy and light chains. Nucleic Acids Res. 19:4133-4137.

32) Horton, R. M., H. D. Hunt, S. N. Ho, J. K. Pullen, and L. R. Pease. 1989. Engineering hybrid genes without the use of restriction enzymes: gene splicing by overlap extension. Gene 77:61-68.

33) Huston, J. S., D. Levinson, H. M. Mudgett, M. S. Tai, J. Novotny, M. N. Margolies, R. J. Ridge, R. E. Bruccoleri, E. Haber, R. Crea, and H. Opperman. 1988. Protein engineering of antibody binding sites: recovery of specific activity in an anti-digoxin single-chain Fv analogue produced in Escherichia coli. Proc. Natl. Acad. Sci. U.S.A. 85:5879-5883. 
34) Janvier, B., P. Archinard, B. Mandrand, A. Goudeau, and F. Barin. 1990. Linear B-cell epitopes of the major core protein of human immunodeficiency virus typess 1 and $2 . \mathrm{J}$. Virol. 64:4258-4263.

35) Janvier. B., A. Baillou. P. Archinard. M. Mounier, B. Mandrand, A. Goudeau, and F. Barin. 1941. Immune response to a major epitope of p24 during infection with human immunodeficiency virus type 1 and implications for diagnosis and prognosis. J. Clin. Microbiol. 29:488-492.

.36) Kabat, E. A., T. T. Wu, H. M. Perry, K. S. Gottesman, and C. Foeller. 1991. Sequences of Protcins of Imnunological Interest. 5th Edit. U.S. Department of Health and Human Services, Public Health Service National Institutes of Health, Bethesda, Md.

37) Kazemier, B., H. de Haard. P. Boender, B. van Gemen, and H. R. Hoogenboom. 1996. Determination of active single chain antibody concentrations in crude periplasmic fractions. J. Immunol. Methods 194:201-209.

38) Kellogg, D. E., J. J. Sninsky, and S. Kowk. 1990. Quantitation of HIV-1 proviral DNA relative to cellular DNA by the polymerase chain reaction. Anal Biochem. 189:202-208.

39) Kortt, A. A., M. Lah, G. W. Oddie, C. L. Gruen, J. E. Burns, L. A. Pearce, J. L. Atwell, A. J. McCoy, G. J. Howlett, D. W. Metzger, R. G. Webster, and P. J. Hudson. 1997. Single-chain Fv fragments of anti-neuraminidase antibody NC10 containing live- and ten-residue linkers form dimers and with zero-residue linker a trimer. Protein Eng. 10:423-433.

40) Lew, A. M. 1984. The effect of epitope density and antibody affinity on the ELISA as analysed by monoclonal antibodies. J. Immunol. Methods 72:171-176.

4l) Luban, J., K. L. Bossolt, E. K. Franke, G. V. Kalpana, and S. P. Goff. 1993. Human immunodeficiency virus type 1 Gag protein binds to cyclophilins A and B. Cell 73:10671078.

42) Marks, J. D., A. D. Griffiths, M. Malmqvist. T. P. Clackson, J. M. Bye, and G. Winter. 1992. By-passing immunization: Building high affinity human antibodies by chain shuffling. Bio/Technology 10:779-783.

43) Marks, J. D., H. R. Hoogenboom, T. P. Bonnert, J. McCafferty, A. D. Griffiths, and G. Winter. 1991. By-passing immunization: Human antibodies from V-gene libraries displayed on phage. J. Mol. Biol. 222:581-597.

44) McGuinness, B. T., G. Walter, K. FitzGerald, P. Schuler, W. Mahoney, A. R. Duncan, and H. R. Hougenboom. 1996. Phage diabody repertoires for selection of large numbers of bispecific antibody fragments. Nature Biotechnol. 14:1149-1154.

45) Mhashilkar. A. M., J. Bagley. S. Y. Chen, A. M. Szilvay, D. G. Helland, and W. A. Marasco. 1995. Inhibition of HIV-1 Tat-mediated LTR transactivation and HIV-1 infection by antiTat single chain intrabodies. EMBO J. 14:1542-1551.

46) Momany. C.. L. C. Kovari. A. J. Prongay, W. Keller. R. K. Gitti, B. M. Lee, A. E. Gorbalenya. L. Tong, J. McClure, L. S. Ehrlich, M. F. Summers, C. Carter, and M. G. 
Rossmann. 1996. Crystal structure of dimeric HIV-1 capsid protein. Nat. Struct. Biol. 3:763770 .

47) Nieba, L., A. Krebber, and A. Pluckthun. 1996. Competition BlAcore for measuring true affinities: large differences from values determined from binding kinetics. Anal. Biochem. 234:155-165.

48) Niedrig, M., J. Hinkula, H. P. Harthus, M. Broker, L. Hopp, G. Pauli, and B. Wahren. 1991. Characterization of murine monoclonal antibodies directed against the core proteins of human immunodeficiency virus types 1 and 2. J. Virol. 65:4529-4533.

49) Niedrig, M., J. Hinkula, W. Weigelt, J. L'Age-Stehr, G. Pauli, J. Rosen, and B. Wahren. 1989. Epitope mapping of monoclonal antibodies against human immunodeficiency virus type 1 structural proteins by using peptides. J. Virol. 63:3525-3528.

50) Niedrig, M., J. P. Rabanus, J. L'Age Stehr, H. R. Gelderblom, and G. Pauli. 1988. Monoclonal antibodies directed against human immunodeficiency virus (HIV) gag protcins with specificity for conserved epitopes in HIV-1, HIV-2 and simian immunodeliciency virus. J. Gen. Virol. 69:2109-2114.

51) Pluckthun, A., and P. Pack. 1997. New protein engineering approaches to multivalent and bispecific antibody fragments. Immunotechnology 3:83-105.

52) Popovic, M., P. S. Sarin, M. K. Robert-Gurroff, V. S., Kalyanaraman, D. Mann, J. Minowada, and R. C. Gallo. 1983. Isolation and transmission of human retrovirus (human tcell leukemia virus). Science 219:856-859.

53) Prickett, K. S., D. C. Amberg, and T. P. Hopp. 1989. A calcium-dependent antibody for identification and purification of recombinant proteins. Biotechniques 7:580-589.

54) Robert-Hebmann, V., S. Emiliani, F. Jean, M. Resnicoff, F. Traincard, and C. Devaux. 1992. Clonal analysis of murine $B$ cell response to the human immunodeficiency virus type 1 (HIV1)-gag p17 and p25 antigens. Mol. Immunol. 29:729-738.

55) Robert-Hebmann, V., S. Emiliani, M. Resnicoff, F. Jean, and C. Devaux. 1992. Subtyping of human immunodeficiency virus isolates with a panel of monoclonal antibodies: identification of conserved and divergent epitopes on p17 and p25 core proteins. Mol. Immunol. 29:1175-1183.

56) Schier, R., J. Bye, G. Apell, A. McCall, G. P. Adams, M. Malmqvist, L. M. Weiner, and J. D. Marks. 1996. Isolation of high-affinity monomeric human anti-c-erbB-2 single chain Fv using affinity-driven selection. J. Mol. Biol. 255:28-43.

57) Schier, R., A. McCall, G. P. Adams, K. W. Marshall, H. Mertitt. M. Yim, R. S. Crawford, L. M. Weiner, C. Marks, and J. D. Marks. 1996. Isolation of picomolar affinity anti-c-erbB-2 single-chain Fv by molecular evolution of the complementarity determining regions in the center of the antibody binding site. J. Mol. Biol. 263:551-567. 
58) Skerra, A., and A. Pluckthun. 1991. Secretion and in vivo folding of the Fab fragment of the antibody McPC603 in Escherichia coli: influence of disulphides and cis-prolines. Protein Eng. 4:971-979.

59) Spector, S. A., C. Kennedy, J. A. McCutchan, S. A. Bozzette, R. G. Straube, J. D. Connor, and D. D. Richman. 1989. The antiviral effect of zidovudine and ribavirin in clinical trials and the use of p24 antigen levels as a virologic marker. J. Infect. Dis. 159:822-828.

60) Steinkasserer, A. R. Harrison, A. Billich, F. Harnmerschmid, G. Werner, B. Wolff, P. Peichl, G. Palti, W. Schnitzel. E. Mlynar, and et. al. 1995. Mode of action of SDZ NIM 811, a nonimmunosuppressive cyclosporin $\mathrm{A}$ analog with activity against human immunodeficiency virus type I (HIV-1): interference with early and late events in HIV-1 replication. J. Virol. 69:814-824.

61) Stevens, P. W., C. H. Francis, M. E. Jolley, and D. R. Bethell. 1993. Monoclonal antibodies to HIV-1 p24 core protein include pairs which exhibit synergistic binding. Mol. Immunol. 30:243-254.

62) van Gemen, B., T. Kievits, P. Nara, H. G. Huisman, S. Jurriaans, J. Goudsmit, and P. Lens. 1993. Qualitative and quantitative detection of HIV-1 RNA by nucleic acid sequence-based amplification. AIDS 7 Suppl 2:S107-S110.

63) Veronesc, F. D., T. D. Copeland, S. Oroszlan, R. C. Gallu, and M. G. Sarngadharan. 1988. Biochemical and immunological analysis of human immunodeficiency virus gag gene products p17 and p24. J. Virol. 62:795-801.

(64) Volberding, P. A., S. W. Lagakos, M. A. Koch, C. Pettinelli, M. W. Myers, D. K. Booth, H. H. J. Ballour, R. C. Reichman, J. A. Bartlett. M. S. Hirsch, and et. al. 1990. Zidovudine in asymptomatic human immunodeficiency virus infection. A controlled trial in persons with fewer than 500 CD4-positive cells per cubic millimeter. The AIDS Clinical Trials Group of the National Institute of Allergy and Infectious Diseases. N. Engl. J. Med. 322:941-949.

65) Von Sydow, M., H. Gaines. A. Sonnerborg, M. Forsgren, P. O. Pehrson, and O. Strannegard. 1988. Antigen detection in primary HIV infection. Br. Med. J. (Clin. Res. Ed.) 296:238-240.

66) Wehrly, K., and B. Chesebro. 1997. p24 antigen capture assay for quantification of human immunodeficiency virus using readily available inexpensive reagents. Methods 12:288-293.

67) Winter, G., A. D. Griffiths, R. E. Hawkins, and H. R. Hoogenboom. 1994. Making antibody by phage display technology. Ann. Rev. Immunol. 12:433-455.

68) Winter, G., and C. Milstein. 1991. Man-made antibodies. Nature 349:293-299.

69) Zhao, Y., Y. Chen, M. Schutkowski, G. Fischer, and H. Ke. 1997. Cyclophilin A complexed with a fragment of HIV-l gag protein: insights into HIV-1 infectious activity. Structure 5:139-146. 


\section{ChAPTER 6}

\section{SELECTION OF HUMAN ANTI- HIV-1 ENVELOPE SINGLE CHAIN ANTIBODIES}

Published as

Selection of human anti- human immunodeficiency virus type 1 envelope single-chain antibodies from a peripheral blood cell-based phage repertoire

Hans J.W. de Haard, Bert Kazemier, Peter Oudshoorn, Piet Boender, Bob van Gemen, Marck J. M. Koolen, Guido van der Groen, Hennie R.J.M. Hoogenboom and Jan-Willem Arends

Journal of General Virology

1998; Volume 79, pages 2883 - 2894 


\section{Abstract}

Monoclonal antibodies play an important role in the development of diagnostic assays. Instead of using hybridoma technology to isolate human immunodeficiency virus type 1-specific antibodies, a phage-displayed antibody library was generated from a small number $\left(10^{7}\right)$ of peripheral blood lymphocytes from a seropositive donor. Two families of single-chain antibodies (scFvs) were selected by biopanning with the envelope precursor gp 160. ELISA and competition in the BIAcore system revealed that one antibody family recognized a conformation-sensitive epitope within gp 120, while the other antibody family was gp41-specific. The latter group had sequence similariy to antibodies recognizing the cluster III epitope of gp41. Binding of scFvs to gp 160 could be inhibited with the donor's serum antibodies, indicating that antibodies with a similar specificity were circulating in the donor's blood. Competition experiments suggested that the epitope of the anti-gp4l antibodies was recognized by a broad range of patients' sera: 21 out of 22 sera from North American and all 20 sera from African seropositive patients inhibited binding of scFvs. In contrast, three sera from this panel did not react with the epitope of the antigp120 antibodies. These data indicate that, because of the conserved nature of its epitope, the anti-gp4 1 antibody will be suitable for diagnostic applications.

\section{Introduction}

Diagnosis of human immunodeficiency virus (HIV) infection by in vitro assays is performed by the detection of virus-specific antibodies or by detection of the virus itself, by means of associated antigens or by its genome and transcribed genes. Virus directed antibodies cannot be detected with currently used assays until 6 to 8 weeks after infection; detection of antigen in immunoassays or viral genes in nucleic acid-based tests shortens this so-called 'window' phase (Busch \& Alter, 1995). Due of their high specificity, monoclonal antibodies are used for the capture and detection of antigens. These antibodies should be of high affinity and be directed against a conserved epitope. The affinity, which is directly related to the sensitivity of the assay (Lew, 1984; Devey \& Steward, 1988), will determine the earliest time point of diagnosis. Also, the epitope of the antibody must be conserved, since the heterogeneity of the virus and its high mutation rate may lead to the complete failure of assays, as was recently found with sera from individuals infected with the subtype O virus (Gurtler, 1996).

In addition to diagnosis of infection, immunoassays could be used for monitoring therapy. For instance. during treatment of patients with nucleoside reverse transcriptase inhibitors or protease inhibitors alone (Spector et al.. 1989; Fischl et al., 1990; Volberding et al.. 1990) or in combination (Harindra, 1997), replication of the virus is currently followed by CD4 counts and 
the measurement of virus load by amplification or hybridisation methods. However, HIV treatment budgets are under considerable pressure. Therefore, a very sensitive immunoassay (Boni et al., 1997) for the detection of the capsid protein (Von Sydow et al., 1988; Graziosi et al., 1993; Baumberger et al., 1993) would be an attractive and cheap alternative to more expensive molecular diagnostics. When resistance occurs, demonstrated by a re-activation of the virus, treatment can be changed to another combination of drugs.

Immunoassays may also be used for prognosis and treatment strategy planning. The quantification of anti-gp41 and anti-p24 antibodies aids in the determination of the stage of the disease. During HIV pathogenesis, the titre of anti-gp4l antibodies remains more or less stable, while the titre of $\alpha$-p24 antibodies gradually declines. Therefore, the ratio between these titres has prognostic value and can determine the appropriate treatment (Schmidt et al., 1989; Strathdee et al., 1995; Binley et al., 1997). To allow quantification, monoclonal antibodies directed against p24 have been labelled with a reporter enzyme and used to compete with serum antibodies for antigen binding (Janvier ct al., 1991).

In this study we have evaluated phage antibody library technology (reviewed in de Haard $e t$ al., 1998a) as a substitute for the traditional hybridoma technology. Theoretically, there are several advantages in applying phage-display technology, and patient-derived human antibodies in particular, to the generation of diagnostic reagents. Firstly, the recognition of immunodominant epitopes is crucial when antibodies are used in antibody-quantification assays. The immunization of mice does not necessarily result in the isolation of monoclonal antibodies which share the immunodominant epitopes recognized by human antibodies. Moreover, the presentation of the viral antigens during immunization might be different from the situation during infection, leading to the isolation of antibodies with different recognition patterns. Secondly, the conserved nature of the epitope is especially important in antigen detection. The existence of HIV variants and the mutational drift of the virus, which occurs in response to the appearance of neutralizing antibodies during infection (Watkins et al., 1996), causes a high variability in all virus genes and their encoded proteins. The use of antigens from different virus strains in the biopanning procedure might favour the selection of such neutralizing antibodies. Thirdly, the application of human antibodies in antigen assays may avoid false-positive reactions caused by sera with human anti-mouse reactivity (HAMA); these sera were found sporadically during the development of a 224 -antigen assay. Use of human scFv-fragments would even prevent problems with human anti-human responses (HAHA). Finally, the availability of the $\mathrm{V}$ genes permits the manipulation of anytibody affinity (reviewed by Hoogenboom, 1997).

In this study we set out to generate human recombinant antibodies reactive against HIV-1, aiming mainly at their application as diagnostic reagents. As a source of antibody producing cells, a small number of circulating lymphocytes from a seropositive individual were used for the construction of the immune library. Biopanning was performed with the envelope precursor glycoprotein gpl60, which is processed into the exterior envelope protein gpl20 and the 
transmembrane glycoprotein gp41. In addition to a lamily of gp 120-specilic antibodies, we selected a group of gp41-specific antibodies with a similar sequence to the cluster III epitoperecognizing antibodies isolated by Binley et al. (1997). The potential for both families of scFvs as diagnostic reagents was examined by competition with a panel of patients' sera, that could be expected to be diverse with respect to virus subtype. Only one serum, which gave unreliable results in other HIV-1 assays, did not recognize the gp4l epitope, while three sera failed to react with the gp120-epitope. Therefore, the anti-gp41 scFv seems to be the most promising candidate for application in in vitro diagnostics.

\section{Materials and methods}

\subsection{Library construction and selections}

Blood cells from a seropositive individual were separated on Ficoll Hipaque (Pharmacia) and stored in aliquots of $10^{7}$ cells per vial in liquid nitrogen. For RNA isolation the cells from one vial were pelleted and washed with physiological salt solution. Approximately $2 \mu \mathrm{g}$ total RNA was extracted (Chirgwin et al., 1979). One $\mu \mathrm{g}$ was reverse transcribed into random-primed cDNA. IgG-specific variable heavy (VH) chain fragments were amplified using Amplitaq polymerase (Perkin-Elmer) for 30 cycles $\left(94^{\circ} \mathrm{C}\right.$ for $30 \mathrm{sec}, 55^{\circ} \mathrm{C}$ for $30 \mathrm{sec}$ and $72{ }^{\circ} \mathrm{C}$ for 2 min) with HuVHBACK and HuIgGl-4CHIFOR primers (for primer sequences see Marks et al., 1991). We combined the highly similar primers VHla, VH3a and VH5a into one pool, VH4a and VH6a into a second group, and kept VH2a separately. In this way three different heavy chain amplification mixtures were obtained. The kappa light chain variable (VK) domains were amplified with the HuVKBACK and HuCKFOR primers. Primers VKla and VK4a were combined, VK2a, VK3a and VK6a formed a second group, and VK5a was kept separate. The three heavy chain and three light chain fragments were isolated from a $1.5 \%$ agarose gel with the QIAex kit (QIAgen) and reamplified with the corresponding J primers (HuJH1 and JH2 combined with JH4 and JH5, HuJH3 with JH6. HuJK 1 combined with JK2, JK3 and JK4, while JK5 formed a separate group). We assembled the six heavy and six light chain fragments in PCR (Horton et al., 1989) with the corresponding linkers into 36 fusion products. These were gelpurified, digested with Sfil and NotI (Pharmacia). and cloned into the vector $\mathrm{pV}$, which is identical to pHENI (Hoogenboom et al., 1991) except for the substitution of a FLAG-tag (Hopp et al., 1988) for the Myc-tag (Evan et al., 1985). Electroporation into E. coli JMI0l was performed as described elsewhere (Dower et al., 1988). To establish the degree of variation of the library, a BsiNI fingerprint (New England Biolabs) was performed on the PCR products of 24 individual clones (Marks ef al., 1991). All gave unique pattems, suggesting that no overrepresented fragments were amplified and cloned.

The rescue of phagemid particles was performed as in Marks et al. (1991) except that for panning. $100 \mu \mathrm{l} 0.45 \mu \mathrm{M}$-filtered culture supernatant (approx. $3 \times 10^{10}$ transforming units) was 
diluted with $900 \mu \mathrm{l}$ sample diluent (PBS, 20\% normal goat serum, 1.0\% Triton X-100). The library was selected with the envelope precursor gp 160. derived from the HIV-1 subtype III $_{B}$ secreting cell line 6D545i (Kalyanaraman et al., 1988), and purified by affinity chromatography with an anti-gp41 monoclonal antibody as described before (Kalyanaraman et al., 1990), and with the Gag-protein p24. The p24 antigen, expressed as full-length product in $E$. coli under control of the inducible lac promoter (constructed at Organon Teknika by M. Berman, unpublished results) and encoding the HIV-1 RF sequence, was affinity-purified with monoclonal antibody mAb 39B (generated at Organon Teknika, unpublished results). The proteins were coated as a mixture in 6-well tissue culture plates (Costar), at a concentration of $2.5 \mu \mathrm{g} / \mathrm{ml}$ for gpl60 and $3 \mu \mathrm{g} / \mathrm{ml}$ for $\mathrm{p} 24$, in $50 \mathrm{mM} \mathrm{Na}_{2} \mathrm{CO}_{3} \mathrm{pH} 9.6$ for $16 \mathrm{~h}$ at $4{ }^{\circ} \mathrm{C}$.

\subsection{Screening and sequencing of clones}

Soluble scFv was produced from individual clones by growth in V-shaped wells of microtitre plates, as described before (Marks et al., 1991). For ELISA, microtitie plates were coated with $3 \mu \mathrm{g} / \mathrm{ml} \mathrm{p} 24,2.5 \mu \mathrm{g} / \mathrm{ml}$ gp 160 or $5 \mu \mathrm{g} / \mathrm{ml}$ virus lysate, in $50 \mathrm{mM} \mathrm{Na} \mathrm{CO}_{3} \mathrm{pH} 9.6$ for $16 \mathrm{~h}$ at $4{ }^{\circ} \mathrm{C}$. The plates were blocked for $2 \mathrm{~h}$ with $0.2 \%(\mathrm{w} / \mathrm{v}) \mathrm{BSA}$ in $0.1 \mathrm{M}$ Tris- $\mathrm{HCl} \mathrm{pH} 7.4$ plus $30 \mathrm{mM} \mathrm{KI}$. Culture supernatants and periplasmic fractions were diluted in sample diluent. After incubation at room temperature for $2 \mathrm{~h}$, bound scFv was detected with anti-FLAG antibody M2 (Kodak IBI), diluted 1:4000 in sample diluent. After a $1 \mathrm{~h}$ incubation with 1:2000 diluted anti-mouse IgG-horseradish peroxidase conjugate (anti-mouse-HRP, DAKO), staining was performed for $30 \mathrm{~min}$ with tetramethylbenzidine (TMB) and urea peroxide (Organon Teknika) as substrates. The reaction was stopped by adding an equal volume $1 \mathrm{M} \mathrm{H}_{2} \mathrm{SO}_{4}$; the absorbance was measured at $450 \mathrm{~nm}$.

Clones giving positive signals and with a unique BstNI fingerprint were analysed by sequencing using the $\mathrm{T} 7$ sequencing kit (Pharmacia) with the M13rev primer (5' CAGGAAACAGCTATGAC 3'), the gene III primer (5' TGAATTTTCTGTATGAGG $3^{\prime}$ ) and two primers located in the linker (LINKFWD, 5' CTCGGGCGGTGGTGGGTC 3'; LINKREV, 5' GACCCACCACCGCCCGAG 3'). Plasmid DNA was purified with the QIAgen kit (QIAgen).

\subsection{Characterization of scFvs}

Periplasmic fractions were prepared with borate-buffered saline (BBS) (Skerra \& Pluckthun, 1991). The cells from a $50 \mathrm{ml}$ culture were resuspended in $1 \mathrm{ml} \mathrm{BBS} \mathrm{(200} \mathrm{mM}$ boric acid-NaOH pH8.0. $160 \mathrm{mM} \mathrm{NaCl}$ ). After 2 to $16 \mathrm{~h}$ incubation at $4^{\circ} \mathrm{C}$, spheroplasts were pelleted by a differential centrifugation step: $10 \mathrm{~min}$ at $6000 \mathrm{~g}$ in an Eppendorf centrifuge followed by an additional clearance of the supernatant at $13000 \mathrm{~g}$ for $30 \mathrm{~min}$.

Virus lysate. BSA and gp 160 were loaded onto $12 \%$ SDS-PAGE gels and blotted onto nitrocellulose. After blocking with a skimmed milk solution ( $5 \% \mathrm{w} / \mathrm{v}$, in TBS), periplasmic fractions were added to the solution $(10 \% \mathrm{v} / \mathrm{v})$. Bound scFv was detected subsequently with anti- 
FLAG M2, diluted 1:4000 in TBS plus Triton X-100, and with anti-mouse alkaline phosphatase (anti-mouse-AP, Promega), diluted 1:4000 in TBS.

The specificity of the scFvs was examined by ELISA with immunopurified gp 120 (Kalyanaraman et al., 1990), a constrained V3 loop with the sequence CTRPNNNTRKSIHIGPGRAFYTTGEIIGDIRQAHC (Tolman et al., 1993) and a constrained gp41-derived peptide with the sequence RILAVERYLKDQQLLGIWGCSGKLIC (Gnann et al., 1987) ats antigens.

For competition ELISA, plates were coated with gp 160 as described above. For inhibition with serum antibodies from the donor of the peripheral blood lymphocytes (PBL) used for library construction, a fixed concentration of scFv from clones AB\#31 and AB\#32 was applied (1:1000- and 1:750-diluted periplasmic fraction, respectively), while the serum was diluted $1: 100,1: 1000$ and 1:10000. For competition with heterologous sera, a panel was tested containing samples from 22 North American (Mexico and USA) and 20 African (Cameroon, Ivory Coast and Tanzania) HIV-1 infected individuals. The scFvs, diluted as above, and the serum, 50-fold diluted, were incubated simultaneously; scFv bound to gp 160 was detected with $\alpha-F L A G$ and anti-mouse-HRP.

Epitope mapping was performed with BIAcore (Malmborg \& Borrebaeck, 1995). gp 160 was coupled covalently to a CM-chip (BIAcore AB) according to the supplier's instructions. The different classes of gp 160-binding scFv were injected sequentially, yielding equilibrium signals. Antibody fragments recognizing different epitopes should give additional responses, whereas an unchanged signal suggests the recognition of a common epitope.

Affinities were determined by kinetic analysis with surface plasmon resonance (BlAcore) using a CM5-chip containing 2856 response units (RU) immobilized gp 160. Three and four different concentrations of $s \mathrm{cFv}$ were injected for $\mathrm{AB} \# 31$ and $\mathrm{AB} \# 32$, respectively. The on-rate was calculated at the concentration of $\mathrm{scFv}$ present in periplasmic fractions, which was determined by densitometric measurement of anti-FLAG M2-incubated Western blots, with a purified murine anti-chorionic gonadotrophin scFv as a standard (see also de Haard et al., 1998c).

\section{Results}

\subsection{Library construction and panning}

For the generation of a random combinatorial library, we extracted RNA from PBL of a seropositive individual. The donor, a haemophilia sufferer, was infected with HIV-1 in 1981 by a blood-derived coagulation product originating in the USA. The patient's seroconversion was demonstrated by Western blot (Fig. 1): a response against the virus core derivatives emerged 23 months before the PBLs were collected (lane 2). Seven months before harvesting, antibodies 
directed against the envelope derivatives gp 120 and gp41 and polymerase derivatives p64, p51 and p31 were present (lane 3). A sample of $10^{7}$ lymphocytes that had been stored in liquid nitrogen for 10 years was used for RNA isolation. The VH and VK chains were amplified and assembled with the linker-encoding DNA fragment into the scFv gene segments. The library obtained consisted of $5 \times 10^{7}$ clones. of which $97 \%$ had a complete scFv gene (not shown).

The library was subjected to four rounds of panning with a combination coat of gpl60 and p24. This selection produced a 5000-fold enrichment in the number of eluted phage particles, relative to the first round. The fraction of phage carrying a complete scFv-encoding insert in the genome was determined by colony morphology (de Haard et al., 1998b): it increased from 60\% after the first round to $98 \%$ after the last round. The polyclonal phage amplification products, which were harvested before each panning round, were tested by ELISA on the combination coat of gp 160 and p24 and also on gp160 alone: both assays gave increasing signals after the second round (data not shown). Twenty-four individual clones picked randomly from the third round and 24 clones from the fourth round were grown in a microtitre plate. The scFvs that leaked into the culture supernatant gave positive reactions against both the combination coat and gpl60 alone and were negative against p24 and BSA. BsiN1-fingerprinting revealed the presence of two different classes of clones. One clone from each of the two classes was analysed in more detail; these were designated $A B \# 31$ and $A B \# 32$.

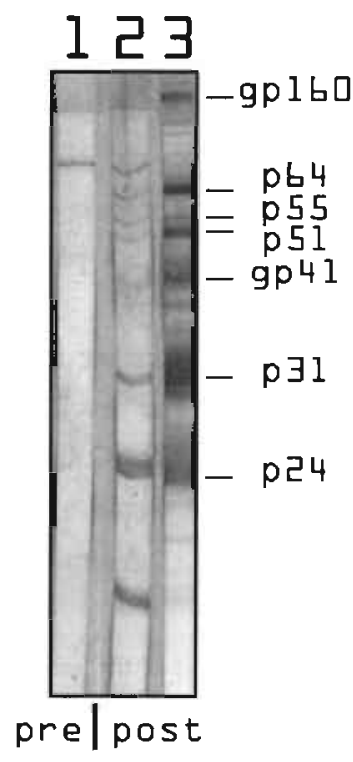

Fig. 1. Analysis of the response of serum from the PBL donor against HIV-1 antigens. Only one band was visible on the strip incubated with pre-infection serum (taken on I October 1981, lane 1), which is a cellular component present in the virus lysate. In lane 2. incubated with serum taken on I May 1982, the characteristics of an early response are visible. i.e. a predominant reactivity against the core derivatives p55, p24 and p17. Lane 3. incubated with a serum sample collected on 23 September 1983. reveals the late response directed mainly directed against the envelope derivatives gp 160 and gp4l and the polymerase products $\mathrm{p} 64, \mathrm{p} 5 \mathrm{i}$ and p31. 
We repeated the panning procedure with gp 160 alone, and found similar degrees of enrichment as when using the combination of antigens. BstNI fingerprints of clones were obtained for antibody fragments that gave positive signals in ELISA; the similar BstNI fingerprints suggested that the clones were closely related to those obtained in previous panning experiment. We examined five new clones, designated \#B4, \#B5, \#B7, \#D1 and \#D4, in more detail.

\subsection{Sequence analysis}

The sequences of the gp 160-binding antibody fragments selected from the human library are shown in Fig. 2. Selection on gpl60 yielded two different types of antibodies with heavily mutated VH genes. Comparison with the germline sequences (Tomlinson et al., 1992) shows that clone $\mathrm{AB} \# 31$ and its derivatives \#B5 and \#B7 use a VH1 family-derived germline segment (DP7). Clone $A B \# 31$ has 30 differences from the germline analogue within its nucleotide sequence, leading to 15 amino acid substitutions. In contrast, clone \#32 and its derivatives \#B4, \#DI and \#D4 use a germline segment of the VH3 family (coded DP-46): for clone AB\#32 there are only 17 differences in its nucleotide sequence resulting in 12 different amino acids. Alignment with the VK germline sequences (Cox et al., 1994) showed that both types of gp 160-specific antibodies use the same segment (VK3 family; coded DPK22/A27) although joined to different $\mathrm{J} \kappa$ segments. The $\mathrm{VK}$ segments are less mutated: only three and seven differences in nucleotide sequence resulting in one and three amino acid substitutions, were observed for clones $A B \# 32$ and $A B \# 31$, respectively.

The use of one type of $\mathrm{VK}$ for both antibodies might indicate a low complexity of the amplified VK-fragments, but a closer examination of the sequences reveals the presence of subtype-specific residues resulting from somatic hypermutation. This can be seen with residue 77 (Fig. 2) and with the J segment-encoded residue at position 96. Ditzel et al. (1997) found a light chain nearly identical to the one used by $A B \# 31$ and $A B \# 32$, but paired with different heavy chain fragments, in their anti-gp 120 antigen-binding fragment (Fab).

The heavy chain CDR3 region of the anti-gpl20 antibodies is rather long (20 residues), while the one from the anti-gp4l antibody is much shorter. The excistence of somatic hypermutation can be identified in the heary chain sequences of the AB\#32 family. The ancestor VH segment, which is found in clone \#B4, contains three germline residues, at position 26, 30 and 56, that are all mutated in the other members of the family (Fig. 2). A similar phenomenon is

Fig. 2 (on next page). Amino acid sequences of the VH and VL. chain regions of the anti-gp 160 antibodies. Numbering of residues and classification of heavy and light chains follows Kabat et al. (1991). Primerencoded difterences within the amino- and earboxy-terminal regions are indicated with lower-case characters. The variable region sequences of the $A B \# 31$ (anti-gp-41) and $A B \# 32$ (anti-gp 120) derivatives are grouped separately with the comesponding germline $\mathrm{V}$ genes. The most similar germline VH segments are DP-7 for $A B \# 31 / \# B 7 / \# B 5$ and $D P-46$ for $A B \# 32 / \# D 4 / \# B 4 / \# D 1$. For VL the germline segment DPK22/A.27 was used in all clones. HS3D6HCV-1 represents the published light chain sequence of an antigp 41 antibody and p 35 indicates a phage library-derived anti-gp 120 Fab. 


\section{A. Heavy chains}

\section{clone \\ Antigen}

AB:31

B. 7

485

$\mathrm{DP} \rightarrow$ ?

$A B=32$

104

B 4

4S3D $6 \mathrm{HCV}-2$

$\operatorname{gp} 41$

OVOLVOSGAEVKKPGASVRVSCKASGHT

CDR1

$40^{\text {ER2 }}$

$502 \mathrm{~A}$

$60 \quad 70 \quad$ FR3

$802 A B C$

90

100ABCDEFG

FR4

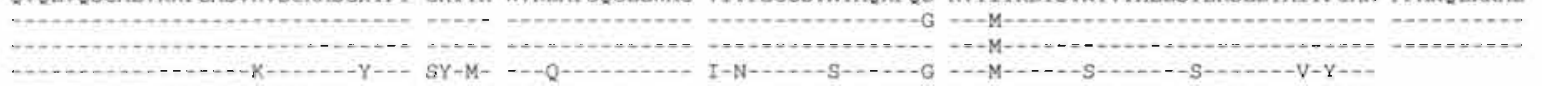

ap 120

EVQLVESGGGVVOPGRSLRLSCAASEFTFN RYDMH WVRQAPGKGLAWVA VISHDGSSKDYADSVRG

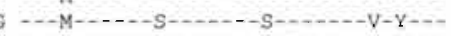


seen in the heavy chain of the AB\#31 family: the germline residues 65 and 69 in clone \#B7 have been mutated in clone $A B \# 31$. Finally, comparison of the sequence of the Vk region from clones \#B6 and \#B7 with $\mathrm{AB} \# 31$ showed that the first two contain ten additional hypermutated residues. At present we do not know if any of these mutations lead to an increase in affinity.

In a comparison of the amino acid sequence of the VH regions using the BLAST program, a similarity was found between the scFv from AB\#32 with a human monoclonal antibody, coded HS3D6HCV-1 (Fig. 2), directed against gp41 (Felgenhauer et al., 1990). A greater homology existed between the $V \kappa$ sequences of $A B \# 31 / A B \# 32$ and the phage library-derived anti-gp120 Fabs p35 and p20 (Ditzel et al., 1997) (see Fig. 2). The heavy chain sequences of p20 and p35 do not resemble those of $s c F v$ from $A B \# 31$ and $A B \# 32$, although they share a long $C D R 3$ region (17 residues for $\mathrm{p} 20$ and $\mathrm{p} 35$ compared with 20 residues for $\mathrm{AB} \# 32$ ) with $\mathrm{AB \# 32}$.

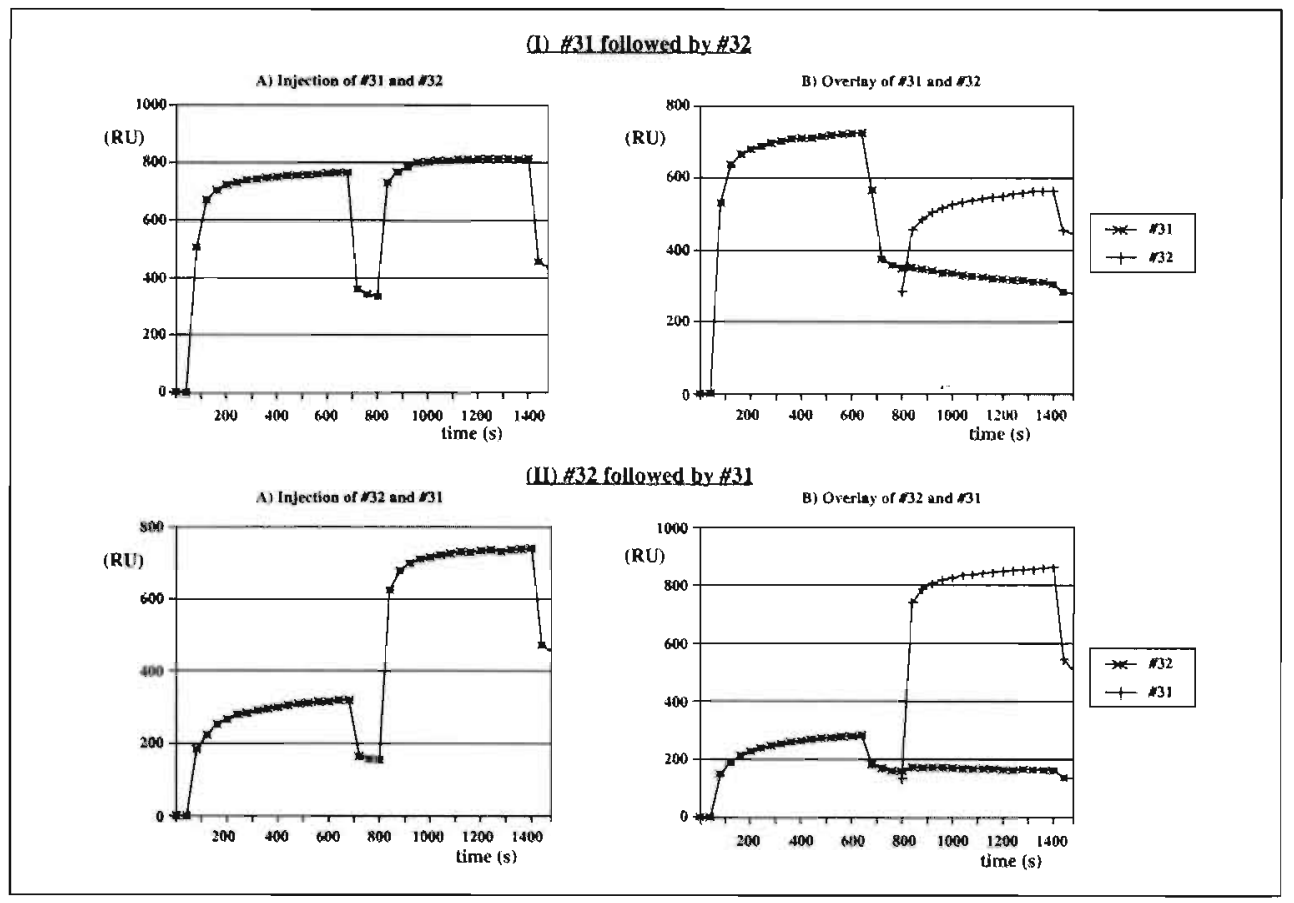

Fig. 3. Epitope mapping with the scFv fragments from clones $A B \# 31$ and $A B \# 32$ on the BIAcore with gp 160 coupled to a CM5-chip at a density of 2856 RU.

Panel LA. After $50 \mathrm{~s}$ the periplasmic fraction of clone AB\#31 was injected, until at $640 \mathrm{~s}$ the Hepes Buffered Saline-wash started. The periplasmic fraction of clone $\# 32$ was injected from $800 \mathrm{~s}$ to $1350 \mathrm{~s}$.

Panel IB. Overlay of the curves from $A B \# 31$ with a prolonged dissociation phase and from $A B \# 32$ [obtained from panel IIB]. The curve of $A B \# 32$ was superimposed at $800 \mathrm{~s}$ on the level for $A B \# 31$ at $1350 \mathrm{~s}$. In this way the loss in signal due to dissociation of $\mathrm{AB} \# 31$ can be corrected, resulting in the observed end-level.

Panel IIA. Sequential injection of $A B \# 32$ and $A B \# 31$ was carried out at the same time points as in (IA). Because of the lower dissociation rate of $A B \# 32$, the increased signal caused by $A B \# 31$ can be recognized more easily. The end-level at $1500 \mathrm{~s}(450 \mathrm{RU})$ is identical to that found in panel IA, showing that the signals are additive.

Panel IIB. Overlay of the curve obtained from $A B \# 32$ with a prolonged dissociation phase, superimposed at $800 \mathrm{~s}$ on that obtained for $\mathrm{AB} \# 31$ at $1350 \mathrm{~s}$ [obtained from IB], confuming additivity. 


\subsection{Characterization of scFvs}

The specificity of both types of antibodies was analysed with BIAcore (Malmborg \& Borrebaeck. 1995). The scFv-containing periplasmic fractions were injected sequentially onto a gp160 surface (Fig. 3). When $A B \# 32$ was injected first, an additional signal was obtained from the subsequent injection of $A B \# 31$ (Fig. 3, panel IIA), suggesting that the antibodies recognize distinct epitopes on gp160. The total signal obtained from both antibodies (at $1500 \mathrm{~s}$ the response was $450 \mathrm{RU}$ ) can be interpreted as the sum of the signals from both scFvs alone [the response of $\mathrm{AB} \# 32$ at $1500 \mathrm{~s}$ was $130 \mathrm{RU}$ (panel IIB) and that of $\mathrm{AB} \# 31$ at $800 \mathrm{~s}$ was $320 \mathrm{RU}$ (panel IA)]. Additivity can be demonstrated by overlaying the individual binding curves (Fig. 3 panel IIB).

The signal obtained by injection of $A B \# 31$ followed by $A B \# 32$ (Fig. 3 panel IA) seems not to be additive, but this appearance is caused by the somewhat faster dissociation of $A B \# 31$. The curve obtained from $A B \# 31$ with a prolonged dissociation phase (Fig. 3, panel IB) shows that the total signal at $1500 \mathrm{~s} \mathrm{(430} \mathrm{RU)} \mathrm{can} \mathrm{also} \mathrm{be} \mathrm{considered} \mathrm{as} \mathrm{the} \mathrm{sum} \mathrm{of} \mathrm{the} \mathrm{signals} \mathrm{of} \mathrm{the} \mathrm{individual}$ antibodies: at $1500 \mathrm{~s}$ the signal of $\mathrm{AB} \# 31$ was $280 \mathrm{RU}$ (Fig. 3, panel IB) and at $800 \mathrm{~s}$ the signal of $A B \# 32$ was $150 \mathrm{RU}$ (Fig. 3, panel ILA).

The affinities of scFv $A B \# 31$ and $A B \# 32$. determined by kinetic analysis of association and dissociation with surface plasmon resonance (Table 1), are in the nanomolar range. $A B \# 32$ has a 12 -fold higher affinity than $A B \# 31$, resulting from a 1.4-fold slower dissociation rate and a 8.6fold faster association rate.

Table 1. Affinities of anti-HIV-I envelope scFvs

\begin{tabular}{lccc}
\hline Clone & $\begin{array}{c}k_{\text {off }} \\
\left(\mathrm{sec}^{-1}\right)\end{array}$ & $\begin{array}{c}\mathrm{k}_{\text {on }} \\
\left(\mathrm{M}^{\mathrm{i}} \cdot \mathrm{sec}^{-1}\right)\end{array}$ & $\begin{array}{c}\mathrm{K}_{\mathrm{d}} \\
(\mathrm{M})\end{array}$ \\
\hline $\mathrm{AB \# 31}$ & $(1.88 \pm 0.43) \times 10^{-3}$ & $(5.38 \pm 0.77) \times 10^{5}$ & $(3.49 \pm 1.29) \times 10^{-9}$ \\
$\mathrm{AB \# 32}$ & $(1.33 \pm 0.10) \times 10^{-3}$ & $(4.64 \pm 1.10) \times 10^{5}$ & $(2.87 \pm 0.89) \times 10^{-10}$ \\
\hline
\end{tabular}

$A B \# 31$ and $A B \# 32$ were used for the detection of HIV antigens on an immunoblot (Fig. 4). Only the non-reduced forms of the envelope protein gp 120 and its precursor gpl60, which is actually a truncated form missing the 68 carboxy-terminal amino acids, were recognized by $\mathrm{AB} \# 32$ [both antigens are marked in Fig. 4A]. AB\#31 was specific for gpl60 and not for gpl20, although the signal on the blot was less intense than that obtained with $A B \# 32$. Omitting boiling of the virus lysate before loading on the gel improved the sensitivity of detection: a more intense gp160, but also a faint gp4l band, were visible on the blot incubated with AB\#31 (Fig. 4B). The bands showing up in the gp 120 preparation are derived from the mouse antibody used for purification, which leaked from the affinity column; it was also detected with the anti-mouse conjugate [see left blot of Fig. 4A]. The proteins reactive with $A B \# 32$ with molecular masses 
greater than $200 \mathrm{kDa}$ are the dimeric products of gp120 and gp160, which were also visible on a blot stained with amido black. In conclusion, $A B \# 31$ recognizes a conformation-sensitive epitope of gp 160, that is probably located in the transmembrane glycoprotein gp41, and AB\#32 reacts with a conformation-sensitive epitope that is present in gp 120 .

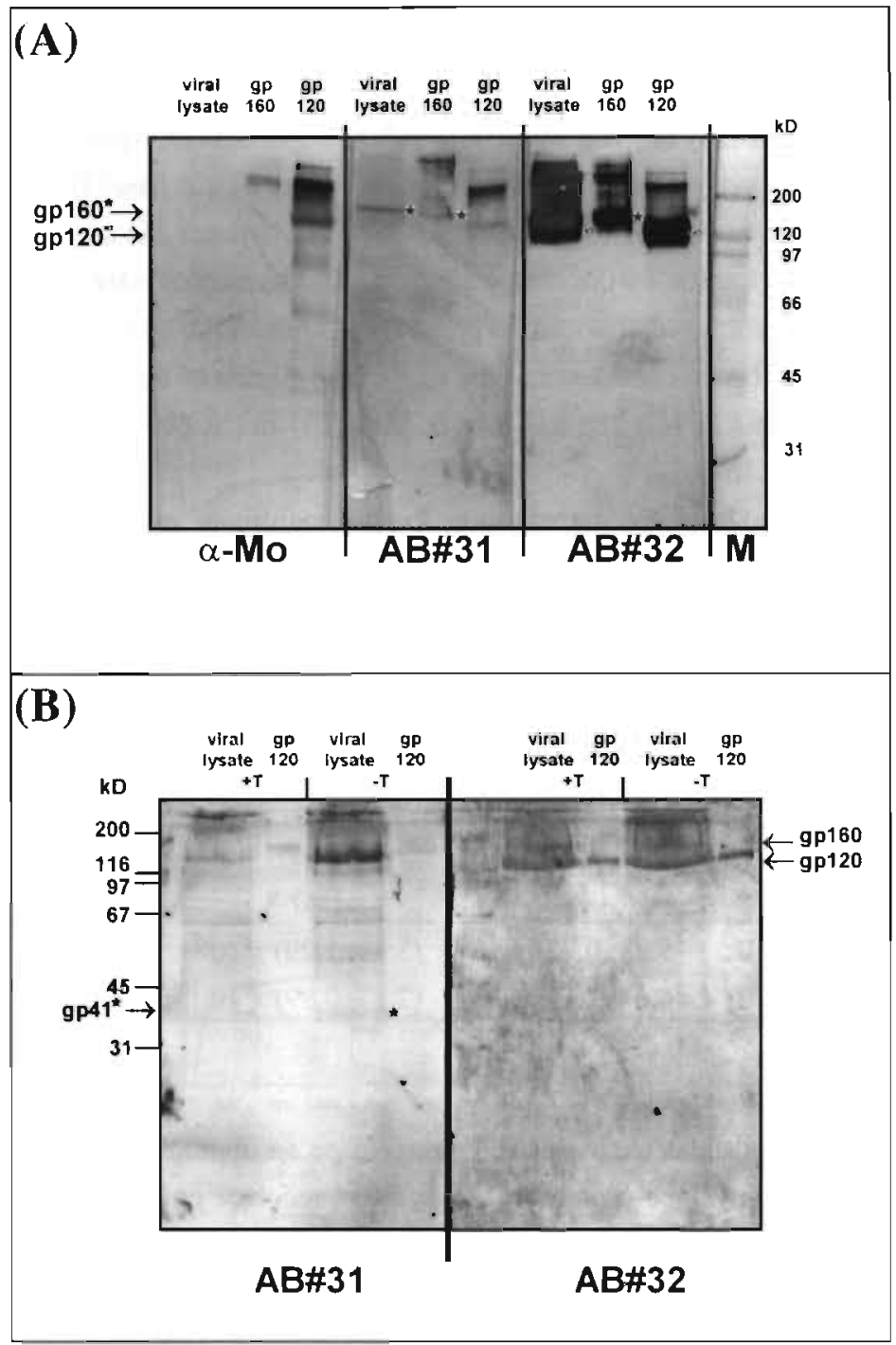

Fig. 4. Analysis of the response of the recombinant antibodies against HIV-1 antigens on a non-reducing Western blot.

(A) Blots were incubated with either anti-mouse-AP ( $\alpha$-Mo), $A B \# 31$ or $A B \# 32$, and the marker lane (M) was stained with Ponceau S. Viral lysate (about $50 \mu \mathrm{g}$ ), purified gpl60 $(2 \mu \mathrm{g})$ and gp120 (2 $\mu \mathrm{g})$ were loaded. The positions of gpl60 and gpl20 are indicated.

(B) Detailed analysis of reactivity of $A B \# 31$ and $A B \# 32$. Virus lysate and gp120 samples were boiled $(+T)$ or loaded directiy on the gel without heat treatment (-T). gp4l was detected by $\mathrm{AB} \# 31$ and not by $\mathrm{AB} \# 32$.

The specificitics of the scFvs from $A B \# 31$ and $A B \# 32$ were analyzed further by ELISA. Immunopurified gp120, a constrained V3 loop peptide of gpl20 (Tolman et al.. 1993) and a constrained peptide of gp4l (Gnann et al., 1987), all based on the HIV-1 sequence. were used to 
coat microtitre plates. A response was only observed for $\mathrm{AB} \# 32$ against gp 120. Therefore, the epitope of $A B \# 31$ must be located within gp41, and it differs from the Gnann epitope. The epitope of AB\#32 resides in gp120, but it is not located within the immunodominant V3 loop.

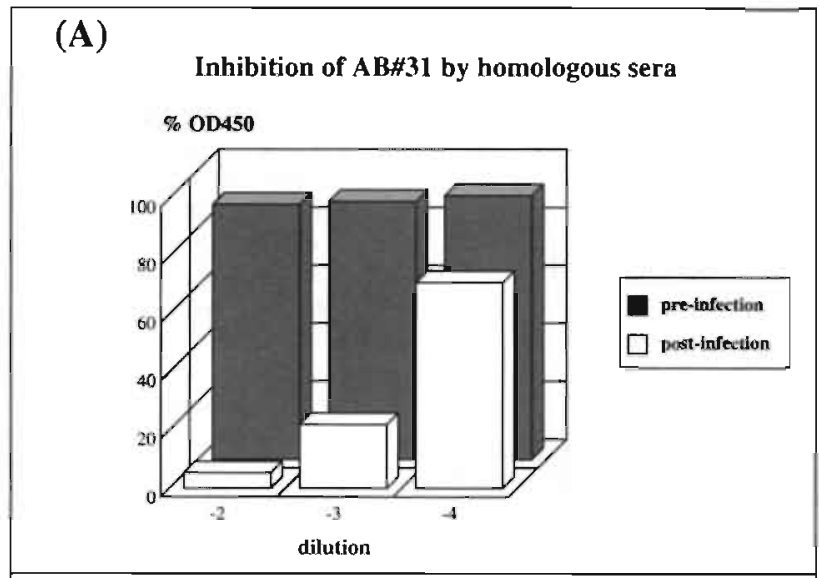

Fig. 5. Inhibition of binding of $A B \# 31$ (A) and $A B \# 32$ (B) to gpl60 in ELISA by serum antibodies from the donor of the PBL, that were used for the construction of the library. Preinfection serum (taken on 19 August 1980, tinted bars) and post-infection serum (taken on 17 September 1983) were diluted 100 -fold $(-2), 1000$-fold $(-3)$ and $10000-$ fold $(-4)$. Results are expressed relative to those obtained with scFv without adding serum.

(B)

Inhibition of $\mathrm{AB} \$ 32$ by homologous sera

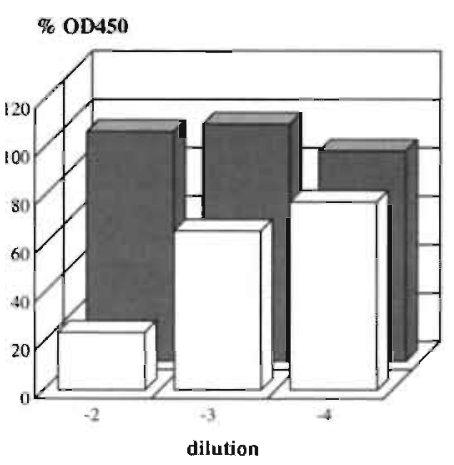

pre-lafection

post-infection

We performed an inhibition ELISA in order to establish the presence of the isolated antibodies in the serum of the donor. The patient's serum, taken before and after HIV infection, the latter with anti-gpl60 reactivity, was mixed with the scFvs and incubated in wells coated with gp 160. Subsequently. bound scFr was detected by its FLAG-tag. As is shown in Fig. 5, the binding of $\mathrm{AB} \# 31$ to the antigen was almost completely inhibited by the serum antibodies: $94.7 \%$ inhibition was observed with a 100 -fold diluted serum and $29.0 \%$ at a 10000 -fold dilution. Binding of $\mathrm{AB} \# 32$ was also competitively inhibited, but to a lesser extent: $76.0 \%$ inhibition was observed at a 100 -fold dilution. In contrast, the pre-infection serum did not affect binding of the recombinant antibodies to gp 160 . This experiment shows that antibodies sharing the epitopes with the library-derived scFvs were present in the serum of the donor. The antibody 
fragments are therefore not artificial products with unusual specificities resulting from the scrambling of VH and VL genes during library assembly.

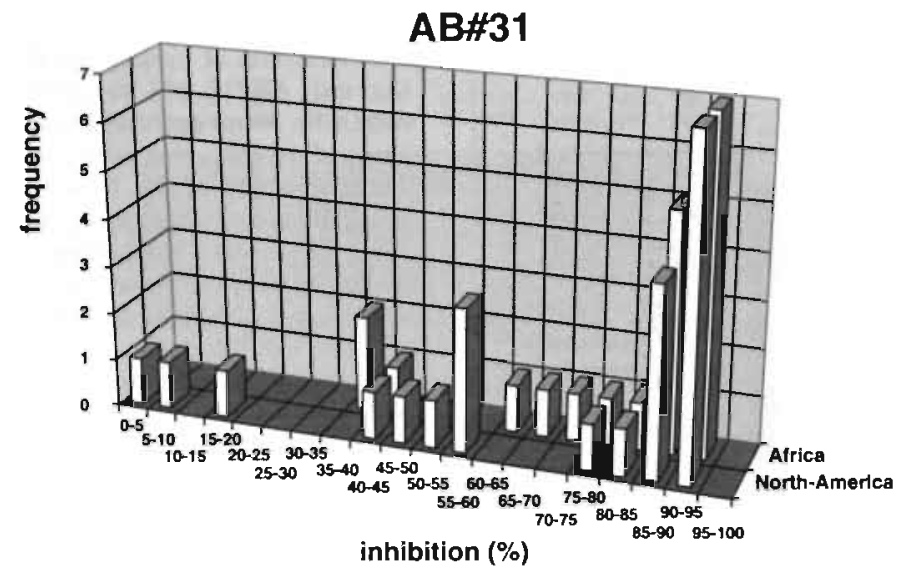

Fig. 6. Competition for binding to gpl60 in ELISA between $A B \# 31$ (above) or $A B \# 32$ (below) and heterologous sera. Three sera did not contain antibodies that competed for binding with $\mathrm{AB} \# 32$ (inhibition $<20 \%$ ). while antibudies from all but one serum competed fior hinding with $\mathrm{AB} \# 31$. Binding by $A B \not 31$ (mean inhibition $95.5 \%, \quad \mathrm{SD} 3.0 \%$ was competed morc efficiently than that by $\mathrm{AB} \# 32$ (African samples. $69.9 \pm 19.9 \%$; North American samples, $86.3 \pm$ 13.6\%).

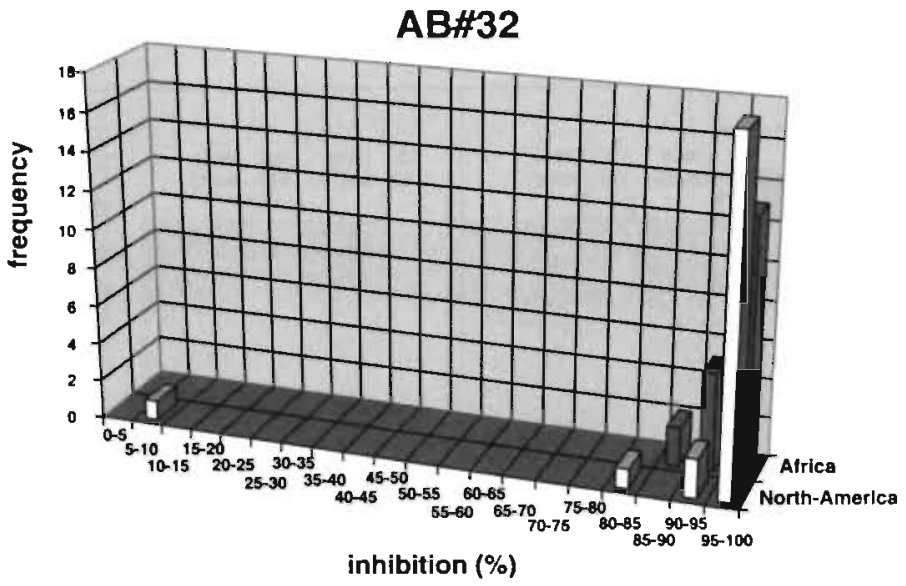

\subsection{Competition with heterologous sera}

To explore the possible application of the scFvs as diagnostic reagents, we analysed the epitope recognition of the selected antibody fragments in more detail. A competition assay was set up, in which a panel of anti-gp 160 positive sera from 22 North American (USA and Mexico) and 20 African (Cameroon. Ivory Coast and Tanzania) patients was tested; the results obtained are shown in Fig. 6. One serum sample from a North American patient showed a low degree of inhibition $(8.4 \%)$ of $\mathrm{AB} \# 31$ binding. The other sera competed well with $\mathrm{AB} \# 31$ for binding to 
gp 160: mean inhibition was $95.5 \%$ with an SD of $1.5 \%$. For $\mathrm{AB}$ \#32 the picture was different. The mean percentage inhibition was much lower (69.9\% with an SD of $19.9 \%$ ), and serum from three North American patients, including the one that did not compete with $A B \# 31$, did not compete for binding. The serum that did not inhibit binding of either scFvs to gpl60 was negative on an immunoblot for binding to p18, p24, p31 and gp41, indeterminate for p51, p55. p65 and gp120 and positive for gp160. The indeterminate and the negative results against gp120 and gp41, respectively. suggest that the signal against gpl60 might be caused by non-specific interactions. This serum gave a borderline signal when tested in the Clonatec assay, which contains a peptide with an immunodominant epitope of gp41. The difference between AB\#31 and $A B \# 32$ in their sensitivity for competition can partly be explained by their affinities: binding of $\mathrm{AB} \# 32$ to gp4l, with a 12-fold lower $K_{d}$ than that of AB\#31 (Table 1), might be inhibited inefficiently.

We conclude that the epitopes of $A B \# 31$ and $A B \# 32$ are recognized by the serum antibodies: from North American and African seropositive patients. This implies that both recombinant antibodies recognize conserved epitopes, thereby making them suitable candidates for application in in vitro diagnostics.

\section{Discussion}

\section{I Small numbers of PBL as a source for library construction}

Combinatorial library technology has already been applied to the generation of human Fabs directed against the HIV-1 envelope (Burton et al., 1991; Barbas et al., 1993; Binley et al., 1997). We selected antibodies from PBL from a haemophilia sufferer (since deceased) who was infected with hepatitis $\mathrm{C}$ virus, and later with HIV-1, through administration of blood-derived coagulation products, and who had no acute immune response at the time of collection of the blood cells. RNA was extracted from a small number of cells $\left(10^{7}\right)$. In spite of the poor condition of the cells, as deduced from their low viability. we managed to isolate a small amount of RNA. After panning of the human library, two types of gp 160-specific antibodies were selected, which have specificities matching serum antibodies from the donor. The two types have different sequences (VH1 and VH3 families), and recognize different epitopes of gp160. The successful selection of these antibodies from a PBL library was surprising, since it has been suggested that the preferred source of antibody-producing B cells is bone marrow (reviewed by Parren \& Burton, 1997). It has been reported that the selection of anti-tetanus toxoid Fabs from PBL libraries was possible from recently boosted donors (Mullinax et al., 1990; Persson et al., 1991), but not from individuals who had not been recently immunized, in spice of the presence of high levels of circulating anti-tetanus toxoid antibodies in their blood (Persson et al., 1991). Most of the circulating lymphocytes are memory cells expressing small amounts of antibody, transcribing 
up to 100 copies of Ig-encoding mRNA per cell, in contrast to plasma cells, which synthesize 30000 copies of Ig mRNA (Schibler et al., 1978). We demonstrated that seroconversion occurred in our donor approximately 30 months before the lymphocytes were collected. One year before collection, the serum contained IgG against gp 120, gp41, p24 and other antigens, as was detected by Western blot. These data suggest that at the time the PBL were harvested the donor did not have an acute response, in which elevated numbers of circulating plasma cells can be expected.

In spite of the presence of anti-p24 antibodies in the donor's serum, we did not retrieve such antibodies from the library during panning with the combination of gp 160 and p24. This might be explained by the low complexity of the library, resulting from the small amount of RNA used for its construction, but it might also be caused by the competitive nature of panning. We believe that the outcome of selection on a mixture of antigens is the antibody with the highest affinity or antibodies with comparable affinities for one of the components, even for the protein used for blocking. This effect has been demonstrated before (Ditzel et al., 1995), during the selection of gp120-specific Fabs. The only way of selecting antibodies that were not reactive against the CD4-binding domain was to shield this epitope through binding to an antibody. Another reason for the absence of anti-p24 scFvs might be a decreasing titre of anti-p24 antibodies in the patient, as is often seen during HIV-1 pathogenesis (Forster et al., 1987; Chargelegue et al., 1993).

The anti-gp120 scFv AB\#32, with a VH3 germline segment, contains a relatively long heavy chain CDR3 regions (20 residues). The library derived anti-gp120 Fab isolated by Barbas and colleagues (Barbas et al., 1993) contained VH1 as well as VH3 segments, with the CDR3-length varying from 18 to 22 residues. No significant similarity was apparent with the VH sequence of our anti-gp120 scFvs. However, there is a most remarkable similarity between the VK sequences of two recently isolated anti-gp120 Fabs (Ditzel et al., 1997) and those of our clones. The light chains of the published Fabs were fused alternatively to the JK segments, leading to a CDR3 of 9 and 10 residues in clone $\mathrm{p} 35$ and $\mathrm{p} 20$, respectively, and they paired with different heavy chains, as was also observed for $\mathrm{AB} \# 31$ and $\mathrm{AB} \# 32$.

\subsection{Possible diagnostic applications}

Our main interest is the application of monoclonal antibodies to in vitro diagnostics. The use of antibodies of human origin in antigen assays prevents false positive reactions caused by sera with HAMA-reactivity. Moreover, the epitopes involved must be conserved and recognized by a broad range of patient's sera. Immunization of mice does not necessarily lead to the isolation of monoclonal antibodies which react with these kinds of epitopes.

The use of the BIAcore system revealed that the selected scFvs react with distinct epitopes on gp160, as was confirmed by ELISA against gp120. AB\#32 reacts with a conformationsensitive epitope of gp 120 different from the V3 loop. In contrast, Western blot analysis with $\mathrm{scFv}$ as the detection antibody suggested that $\mathrm{AB} \# 31$ has gp41-specificity, explaining the 
absence of reactivity of this antibody against gp 120 in ELISA and immunoblot. The epitope recognized by this scFv does not coincide with the immunodominant 'Gnann epitope' (Ginann $\epsilon^{\prime} t$ al., 1987), which is part of the cluster I epitope on gp41. According to the sequence of its VH chain, $A B \# 31$ appears to be related to the cluster III epitope-recognizing recombinant Fabs: described by Binley et al. (1997). Antibodies to this cluster use the same germline VH gene (DP7) and share the CDR3 length (12 residues). A faint sinilarity can be found between $A B \# 31$ and the cluster III clone $G 15$ in the CDR3-encoded [AB\#31. PFMNQLRNAI.DI; G15, PRFNLIRE(A/P)LDL], and both use a VK3 light chain gene. Comparison of clone $A B \# 31$ with G15 revealed 36 differences in the deduced amino acid sequence of $\mathrm{VH}$, but 20 of the differing residues could also be found in one or more of the other cluster III clones G5, L1 l, and in particular in clones L1 and A9 (data not shown). The occurrence of identical somatically mutated residues within CDR1 (residue 31 and 32) and CDR2 (residue 65) in the cluster III clones and clone $A B \# 31$ strengthens the hypothesis of a relation between these antibodies. These data suggest that $A B \# 31$, which like the cluster IIJ Fabs of Binley et al. (1997) only reacts with native antigen on an immunoblot, recognizes a conformational epitope involving residues 619-648 and probably residues 563-583 of gp41 (for numbering see Binley et al., 1997).

Inhibition ELISA with a broad range of anti-gp160-positive sera from seropositive individuals, not only from North America, but also from the Africa, where variability among HIV-1 viruses is much larger than elsewhere (Nkengasong et al., 1994; Louwagie et al., 1995), showed that the epitope on gp41 is immunodominant and conserved. The presence of immunodominant and conserved epitopes on gp4l is well documented (Gnann et al., 1987; Cumming et al., 1990; Muster et al., 1993), and confirms the idea that AB\#31 in particular, which is reactive against this antigen, will be useful for diagnostics.

Besides conservation of its recognized epitope, another important feature of a diagnostic antibody is affinity, since this will determine the sensitivity of the assay (Lew, 1984; Devey \& Steward, 1988). Kinetic measurements with surface plasmon resonance revealed high affinities for both antibodies: $A B \# 31$ has an affinity in the nanomolar range $(3.5 \mathrm{nM})$, while $A B \# 32$ has even greater affinity $(0.29 \mathrm{nM})$,

One diagnostic application of these scFvs could be the quantification of anti-gp4 1 antibodies during HIV-1 pathogenesis. The ratio of anti-p24 to anti-gp4l antibodies has prognostic value: the titre of anti-p24 gradually declines, whereas the anti-gp41 titre remains unchanged (Schmidt et al., 1989; Strathdee et al., 1995; Binley et al., 1997). A quantitative assay for anti-gp4 1 antibodies could be made by construction of labelled scFv AB\#31 that could be used for the competition of serum antibody-binding to immobilized antigen, as has been described before for p24 (Janvier et al., 1991). The format of the scFv can be engineered for this purpose, as has been demonstrated previously (Kerschbaumer et al., 1997). 


\section{References}

Barbas, C. F.. Cullet, T. A., Amberg. W., Roben, P., Binley. J. M., Hoelstra, D., Cababa, D., Jones, T. M., Williamson, A., Piklinlgton, G. R., Haigwood, N. L., Cabezas, E., Satterhwait, A. C., Sanz. I. \& Burton, D. R. (1993). Molecular profile of an antibody response to HIV-1 as probed by combinatorial libraries. J. Mol. Biol. 230, 812-823.

Baumberger, C., Kinloch-de-Loes, S., Yerly. S., Hirschel, B. \& Perrin, L. (1993). High levels of circulating RNA in patients with symptomatic HIV-1 infection. AIDS 7 Suppl 2, S59S64.

Binley, J. M., Klasse, P. J., Cao, Y., Jones, I., Markowitz, M., Ho, D. D. \& Moore, J. P. (1997). Differential regulation of the antibody responses to Gag and Env proteins of human immunodeficiency virus type 1. J. Virol. 71, 2799-2809.

Boni, J., Opravil, M., Tomasik, Z., Rothen, M., Bisset, L., Grob, P. J., Luthy, R. \& Schupbach, J. (1997). Simple monitoring of antiretroviral therapy with a signalamplification-boosted HIV-1 p24 antigen assay with heat-denatured plasma. AIDS 11, F47-F52.

Burton. D. R., Barbas, C. F., Persson, M. A. A., Koenig, S., Chanock, R. M. \& Lerner, R. A. (1991). A large array of human monoclonal antibodies to type 1 human immunodeficiency virus from combinatorial libraries of asymptomatic individuals. Proc. Natl. Acad. Sci. USA 88, 10134-10137.

Busch, M. P. \& Alter, H. J. (1995). Will human immunodeficiency virus p24 antigen screening increase the safety of the blood supply and, if so, at what cost? [editorial]. Transfusion 35, 536-539.

Chargelegue, D., Colvin, B. T. \& CM, O. T. (1993). A 7-year analysis of anti-Gag (p17 and p24) antibodies in HIV-1- seropositive patients with haemophilia: immunoglobulin $G$ titre and avidity are early predictors of clinical course. AIDS 7 Suppl 2, S87-90.

Chirgwin, J. M., Przybyla, A. E., MacDonald, R. J. \& Rutter, W. J. (1979). Isolation of biologically active ribonucleic acid from sources enriched in ribonuclease. Biochemistry 18, 5294-5299.

Cox, J. P. L., Tomlinson, I. M. \& Winter, G. (1994). A directory of human germ-line vк segments reveals a striong bias in their usage. Eur. J. Immunol. 24, 827-836.

Cumming, S. A., McPhee, D. A., Maskill, W. J., Kemp, B. E., Doherty, R. R. \& Gust, I. D. (1990). Use of a conserved immunodominant epitope of HIV surface glycoprotein gp4l in the detection of early antibodies. AIDS 4, 83-86.

de Haard, H., Henderikx, P. \& Hoogenboom, H. R. (1998a). Creating and engineering human antibodies for immunotherapy. Adv. Drug Rev. 31, 5-31.

de Haard, J. J. W., Kazemier, B., Koolen, M. J. M., Nijholt, L. J., Meloen. R. H., Van Gemen, B.. Hoogenboom, H. R. \& Arends, J.-W. (1998b). Selection of recombinant, library derived antibody fragments against p24 for application in HIV-1 diagnostics. Clin. \& Diagn. Lab. Immunol. 5, 636-644.

de Haard, J. J. W., Kazemier. B., van der Bent, A., Oudshoom, P., Boender, P., van Gemen, B., Arends, J.-W. \& Hoogenbonm. H. R. M. (1998c). Absolute Conservation of Residue number 6 of Immunoglobulin Heavy Chain Variable Regions of class IIA is required for correct folding. Protein Eng. 11. 1267-1276.

Devey, M. E. \& Steward, M. W. (1988). The role of antibody affinity in the performance of solid phase assays. In ELISA and Other Solid Phase Immunoassays., pp. 135-153. Edited by D. M. Kemeny \& S. J. Challacombe. London: John Wiley \& Sons Lid. 
Ditzel, H. J., Binley, J. M., Moore, J. P., Sodroski, J., Sullivan, N., Sawyer, L. S., Hendry, R. M., Yang, W. P., Barbas, C. F. \& Burton, D. R. (1995). Neutralizing recombinant human antibodies to a conformational V2- and CD4-binding site-sensitive epitope of HIV-1 gp120 isolated by using an epitope-masking procedure. J. Immunol. 154, 893-906.

Ditzel, H. J., Parren, P. W., Binley, J. M., Sodroski. J., Moore. J. P., Barbas, C. F., 3rd \& Burton, D. R. (1997). Mapping the protein surface of human immunodeficiency virus type 1 gp120 using human monoclonal antibodies from phage display libraries. J. Mol. Biol. 267, 684-695.

Dower, W. J., Miller, J. F. \& Ragsdale, C. W. (1988). High efficiency transtormation of E. coli by high voltage electroporation. Nucleic Acids Res. 16, 6127-6145.

Evan, G. I., Lewis, G. K., Ramsay, G. \& Bishop, J. M. (1985). Isolation of monoclonal antibodies specific for human c-myc proto-oncogene product. Mol. Cell. Biol. 5, 36103616.

Felgenhauer, M., Kohl, J. \& Ruker, F. (1990). Nucleotide sequences of the cDNAs encoding the $\mathrm{V}$-regions of $\mathrm{H}$ - and $\mathrm{L}$-chains of a human monoclonal antibody specific to $\mathrm{HIV}$-1gp41. Nucleic Acids Res. 18, 4927.

Fischl, M. A., Richman, D. D., Hansen, N., Collier, A. C., Carey, J. T., Para, M. F., Hardy, W. D., Dolin, R., Powderly, W. G., Allan, J. D. \& al, e. (1990). The safety and efficacy of zidovudine (AZT) in the treatment of subjects with mildly symptomatic human immunodeficiency virus type 1 (HIV) infection. A double-blind, placebo-controlled trial. The AIDS Clinical Trials Group. Ann Intern Med 112, 727-737.

Forster, S. M., Osborne, L. M., Cheingsong-Popov, R., Kenny, C., Burnell, R., Jeffries, D. J., Pinching, A. J., Harris, J. R. \& Weber, J. N. (1987). Decline of anti-p24 antibody precedes antigenaemia as correlate of prognosis in HIV-1 infection. AIDS 1, 235-240.

Gnann, J. W., Jr., McCormick, J. B., Mitchell, S., Nelson, J. A. \& Oldstone, M. B. (1987). Synthetic peptide immunoassay distinguishes HIV type 1 and HIV type 2 infections. Science 237, 1346-1349.

Graziosi, C., Pantaleo, G., Butini, L., Demarest. J. F., Saag, M. S., Shaw, G. M. \& Fauci, A. S. (1993). Kinetics of human immunodeficiency virus type 1 (HIV-1) DNA and RNA synthesis during primary HIV-1 infection. Proc. Natl. Acad. Sri. USA 90, 6405-6409.

Gurtler, L. (1996). Difficulties and strategies of HIV diagnosis. Lancet 348, 176-179.

Harindra, V. (1997). British HIV Association guidelines for antiretroviral treatment of HIV Seropositive individuals [letter; comment]. Lancet 349, 1838.

Hoogenboom, H. R. (1997). Designing and optimizing library selection strategies for generating high-affinity antibodies. Trends Biotechnol. 15, 62-70.

Hoogenboom, H. R., Griffiths, A. D., Johnson, K. S., Chiswell, D. J., Hudson, P. \& Winter, G. (1991). Multi-subunit proteins on the surface of filamentous phage: methodologies for displaying antibody (Fab) heavy and light chains. Nucleic Acids Res. 19, 4133-4137.

Hopp, T. P., Prickett, K. S., Price, V. L., Libby, R. T., March, C. J., Cerretti, D. P., Urdall, D.

L. \& Conlon, P. J. (1988). A short polypeptide marker sequence useful for recombinant protein identification and purification. BioTechnology (NY) 6, 1204-1210.

Horton, R. M., Hunt, H. D., Ho, S. N., Pullen, J. K. \& Pease, L. R. (1989). Enginecring hybrid genes without the use of restriction enzymes: gene splicing by overlap extension. Gene 77, 61-68.

Janvier, B., Baillou, A., Archinard, P., Mounier, M., Mandrand, B., Goudeau. A. \& Barin, F. (1991). Immune response to a major epitope of p24 during infection with human immunodeficiency virus type 1 and implications for diagnosis and prognosis. J. Clin. Microbiol. 29, 488-492. 
Kabat, E. A., Wu, T. T., Perry, H. M., Gottesman, K. S. \& Foeller, C. (1991). Sequences of Proteins of Immunological Interest. 5th Edit. U.S. Department of Health and Human Services, Public Health Service National Institutes of Health.

Kalyanaraman, V. S., Pal, R., Gallo, R. C. \& Sarngadharan, M. G. (1988). A unique human immunodeficiency virus culture secreting soluble gp160. AIDS Res. Hum. Retroviruses 4, 319-329.

Kalyanaraman, V. S., Rodriguez, V., Veronese, F., Rahman, R., Lusso, P., DeVico, A. L., Copeland, T., Oroszlan, S., Gallo, R. C. \& Sarngadharan, M. G. (1990). Characterization of the secreted, native gp120 and gp 160 of the human immunodeficiency virus type 1. AIDS Res. Hum. Retroviruses 6, 371-380.

Kerschbaumer, R. J., Hirschl, S., Kaufmann, A., Ibl, M., Koenig, R. \& Himmler, G. (1997). Single-chain Fv fusion proteins suitable as coating and detecting reagents in a double antibody sandwich enzyme-linked immunosorbent assay. Anal. Biochem. 249, 219-227.

Lew, A. M. (1984). The effect of epitope density and antibody affinity on the ELISA as analysed by monoclonal antibodies. J. Immunol. Methods 72, 171-176.

Louwagie, J., Janssens, W., Mascola, J., Heyndrickx, L., Hegerich, P., van der Groen, G., McCutchan, F. E. \& Burke, D. S. (1995). Genetic diversity of the envelope glycoprotein from human immunodeficiency virus type 1 isolates of African origin. $J$ Virol 69, 26371.

Malmborg, A. C. \& Borrebaeck, C. A. (1995). BLAcore as a tool in antibody engineering. $J$. Immunol. Methods 183, 7-13.

Marks, J. D., Hoogenboom, H. R., Bonnert, T. P., McCafferty, J., Griffiths, A. D. \& Winter, G. (1991). By-passing immunization: Human antibodies from V-gene libraries displayed on phage. J. Mol. Biol. 222, 581-597.

Mullinax, R. L., Gross, E. A., Amberg, J. R.. Hay, B. N., Hogrefe, H. H., Kubitz, M. M., Greener, A., Alting, M. M., Ardourel, D., Short, J. M. \& et, a. 1. (1990). Identification of human antibody fragment clones specific for tetanus toxoid in a bacteriophage lambda immunoexpression library. Proc. Natl. Acad. Sci. USA 87, 8095-8099.

Muster, T., Steindl, F., Purtscher, M., Trkola, A., Klima, A., Himmler, G., Ruker, F. \& Katinger, H. (1993). A conserved neutralizing epitope on gp4l of human immunodeficiency virus type 1.J. Virol. 67, 6642-6647.

Nkengasong, J. N., Janssens, W., Heyndrickx, L., Fransen, K., Ndumbe, P. M., Motte, J., Leonaers, A., Ngolle, M., Ayuk, J., Piot, P. \& et al. (1994). Genotypic subtypes of HIV1 in Cameroon. AIDS 8, 1405-1412.

Parren, P. W. \& Burton, D. R. (1997). Antibodies against HIV-1 from phage display libraries: mapping of an immune response and progress towards antiviral immunotherapy. Chem. Immunol. 65, 18-56.

Persson, M. A., Caothien, R. H. \& Burton, D. R. (1991). Generation of diverse high-affinity human monoclonal antibodies by repertoire cloning. Proc. Natl. Acad. Sci. USA 88, 2432-2436.

Schibler, U., Marcu, K. B. \& Perry, R. P. (1978). The synthesis and processing of the messenger RNAs specifying heavy and light chain immunoglobulins in MPC-11 cells. Cell 15, 1495-1509.

Schmidt, G., Amiraian, K., Frey, H., Wethers, J., Stevens, R. W. \& Berns, D. S. (1989). Monitoring human immunodeficiency virus type 1 -infected patients by ratio of antibodies to gp41 and p24. J. Clin. Microbiol. 27, 843-848. 
Skerra, A. \& Pluckthun, A. (1991). Secretion and in vivo folding of the Fab fragment of the antibody McPC603 in Escherichia coli: influence of disulphides and cis-prolines. Protein Eng. 4, 971-979.

Spector, S. A., Kennedy, C., McCutchan, J. A., Bozzette, S. A., Straube, R. G., Connor, J. D. \& Richman, D. D. (1989). The antiviral effect of zidovudine and ribavirin in clinical trials and the use of p24 antigen levels as a virologic marker. J. Infect. Dis. 159, 822828.

Strathdee, S. A., Frank, J. W., McLaughlin, J., Leblanc, M., Major, C., MV, O. S. \& Read, S. E. (1995). Quantitative measures of human immunodeficiency virus-specific antibodies predict progression to ADS. J. Infect. Dis. 172, 1375-1379.

Tolman, R. L., Bednarek, M. A., Johnson, B. A., Leanza, W. J., Marburg, S., Underwood, D. J., Emini, E. A. \& Conley, A. J. (1993). Cyclic V3-loop-related HIV-1 conjugate vaccines. Synthesis, conformation and immunological properties. Int. J. Pept. Protein Res. 41, 455-466.

Tomlinson, I. M., Walter, G., Marks, J. D., Llewelyn, M. B. \& Winter, G. (1992). The repertoire of human germline $\mathrm{VH}$ sequences reveals about fifty groups of VH segments with different hypervariable loops. J. Mol. Biol. 227, 776-798.

Volberding, P. A., Lagakos, S. W., Koch, M. A., Pettinelli, C., Myers, M. W., Booth, D. K., Balfour, H. H. J., Reichman, R. C., Bartlett, J. A., Hirsch, M. S. \& al, e. (1990). Zidovudine in asymptomatic human immunodeficiency virus infection. A controlled trial in persons with fewer than $500 \mathrm{CD} 4$-positive cells per cubic millimeter. The AIDS Clinical Trials Group of the National Institute of Allergy and Infectious Diseases. $N$. Engl. J. Med. 322, 941-949.

Von Sydow, M., Gaines, H., Sonnerborg, A., Forsgren, M., Pehrson, P. O. \& Strannegard, O. (1988). Antigen detection in primary HIV infection. Br. Med. J. (Clin. Res. Ed.) 296. 238-240.

Watkins, B. A., Buge, S., Aldrich, K., Davis, A. E., Robinson, J., Reitz, M. S., Jr. \& RobertGuroff, M. (1996). Resistance of human immunodeficiency virus type 1 to neutralization by natural antisera occurs through single amino acid substitutions that cause changes in antibody binding at multiple sites. J. Virol. 70, 8431-8437.

We wish to thank T. Jacobs (Organon Teknika) for providing us with antigens, L. Nijholt and J. Cremers for the gift of sera, and finally N. van Daal and S. Jhagjhoorsingh for technical assistance in library construction. 



\section{Chapter 7}

\section{ANTI- GLYCOPROTEIN HORMONE FAB'S FROM A NAIVE HUMAN REPERTOIRE}

Accepted for publication as

A large naïve human Fab fragment phage library that permits rapid isolation and kinetic analysis of high affinity antibodies

Hans J.W. de Haard, Nicole van Neer, Anneke Reurs, Simon E. Hufton, Rob C. Roovers, Paula Henderikx, Jan-Willem Arends, and Hennie R.J.M. Hoogenboom

Journal of Biological Chemistry 


\section{Abstract}

We report the design, construction and use of the first very large naïve phage antibody library in Fab format, that allows the rapid isolation and affinity-analysis of antigen-specific human antibody fragments. Individually cloned heavy and light chain variable region libraries were combined in an efficient 2-step cloning procedure, permitting the cloning of in total $3.7 \times 1010$ independent Fab clones. The performance of the library was determined by the successful selection of on average 14 different Fab's against 6 antigens tested. These include tetanus toxoid, the hapten phenyl-oxazolone, the breast cancer associated MUC1 antigen and three highly related glycoprotein hormones: human Chorionic Gonadotropin, human Luteinizing Hormone and human Follicle Stimulating Hormone. In the latter category, a panel of either homone-specific or cross-reactive antibodies were identified. The design of the library permits the monitoring of selections with polyclonal phage preparations and to carry out large scale screening of antibody off-rates with unpurified Fab fragments on BlAcore. Antibodies with offrates in the order of 10-2 to 10-4 s-1 and affinities up to $2.7 \mathrm{nM}$, were recovered. The kinetics of these phage antibodies are of the same order of magnitude as antibodies associated with a secondary immune response. This new phage antibody library is set to become a valuable source of antibodies to many different targets, and to play a vital role in target discovery and validation in the area of functional genomics.

\section{Introduction}

Display on filamentous phage in combination with selection forms a powerful tool for the identification of peptide- or protein-based drugs $(1,2)$. Of these, antibodies are especially of interest, due to their capacity to recognize a variety of targets with high specificity and affinity. Particularly the use of partially or completely human antibodies, which elicit no or minimal immune response when administered to patients, is yielding an increasing list of FDA-approved protein-based drugs (3). Phage display technology enables the generation of large repertoires of human antibodies (4-7), while the biopanning procedure permits the selection of individual antibodies with a desired specilicity.

Key to the success of the technology were two critical observations: (i) the expression of functional antibody fragments by secretion into the periplasm of E. coli $(8,9)$, and, (ii) the rapid access to variable region gene pools by the polymerase chain reaction (10-12). For the construction of antibody libraries, V-genes are amplified from B-cell cDNA and heavy and light chain genes are randomly combined and cloned to encode a combinatorial library of single-chain

Fv (scFv) or Fab antibody fragments (4,13-15). The natural primary (unselected) antibody 
repertoire within B-cells contains a large array of antibodies that recognize a variety of antigens; this array can be cloned as a 'naïve' repertoire of rearranged genes, by harvesting the V-genes from the IgM mRNA of B-cells of unimmunized human donors, isolated from peripheral blood lymphocytes (4), bone marrow or tonsils (7), or from similar animal sources (16). This procedure provides access to antibodies that have not yet encountered antigen, although the frequency of those genuine 'germline' antibodies will depend heavily on the source of B-cells (17). A single 'naive' library, if sufficiently large and diverse, can indeed be used to generate antibodies to a large panel of antigens, including self, non-immunogenic and relatively toxic antigens $(6,18)$. In a different approach, antibodies may be built artificially, by in vitro assembly of V-gene segments and D/J segments, yielding 'synthetic' antibodies (5). A major drawback of these procedures is that from the initial 'naive' and 'synthetic' libraries, only moderate affinity antibodies were isolated $(18,19)$. Over the last few years more efficient techniques have heen developed to build larger libraries of antibody fragments, using sophisticated in vivo recombination methods (6) or brute force cloning procedures $(7,20)$. Such large libraries have yielded a greater number of human antibodies per antigen tested, with an average much higher affinity (up to sub-nanomolar). However, technical restrictions on the size of libraries that may be obtained or handled in selection, the loss of library diversity upon library amplification, and the relatively long down-stream analysis path of the selected antibodies, i.e. large scale affinity analysis, have limited the spread of these libraries as generic tools in antibody generation.

We describe here the strategy and construction of a very large phage antibody library, based in a phgemid vector, from which high-affinity human Fab fragments can be selected. The choice for the Fab format was based on the notion that the monomeric appearance of the Fab should permit the rapid screening of large numbers of clones on kinetics of binding (off-rate) with crude protein fractions. This would reduce the time for post-selection analysis dramatically when compared to that needed for selected single-chain Fv (scFv) antibodies from phagemid libraries (7.20), or Fab fragments from phage libraries (6). By applying an efficient cloning strategy, in which restriction fragments instead of PCR-products were used, we generated a repertoire with as many as 37 billion different Fab clones. The performance of the library was analysed by the selection with an extended panel of antigens including three closely related glycoprotein hormones, yielding a diverse set of specific antibody fragments for each antigen. Without using sophisticated selection protocols, hormone specific as well as cross-reactive Fab's were retrieved against the highly homologous glycohormones, demonstrating that the library is a rich source of antibody specificities. The affinities of the anti-glycohormone antibodies varied between 2.7 and 38 nM. Finally, the Fab-format indeed permitted the rapid screening and a reliable ranking of individual clones on off-rate using crude fractions. 


\section{Materials and methods}

\subsection{RNA-isolation}

As source of lymphoid tissues we used peripheral blood lymphocytes from 4 healthy donors and part of a tumor-free spleen removed from a patient with gastric carcinoma. B lymphocytes were isolated from 2-L of blood on a Ficoll-Pacque gradient. For RNA isolation, the cell pellet was immediately dissolved in $50 \mathrm{ml} 8 \mathrm{M}$ guanidinium thiocyanate / $0.1 \mathrm{M}$ 2-mercaptoethanol (21). Chromosomal DNA was sheared to completion by passing through a narrow syringe (1.2/0.5 mm gauge), and insoluble debris was removed by low speed centrifugation (15 min $2,934 \mathrm{xg}$ at room temperature). RNA was pelleted by centrifugation through a CsCl-block gradient $(12 \mathrm{ml}$ supernatant on a layer of $3.5 \mathrm{ml} 5.7 \mathrm{M} \mathrm{CsCl} / 0.1 \mathrm{M} \mathrm{EDTA}$; in total 4 tubes) during $20 \mathrm{~h}$ at $125,000 \mathrm{xg}$ at $20^{\circ} \mathrm{C}$ in a SW41-rotor (Beckman). The yield of total RNA was approx. $600 \mu \mathrm{g}$. RNA was stored at $-20^{\circ} \mathrm{C}$ in ethanol.

From the spleen, $2 \mathrm{~g}$ of tissue was used for homogenisation with a polytron in $20 \mathrm{ml} 8 \mathrm{M}$ guanidinium thiocyanate / $0.1 \mathrm{M} 2$-mercaptoethanol. The total volume was increased to $80 \mathrm{ml}$ with guanidinium thiocyanate buffer, and after passage through a narrow syringe for shearing and removal of debris, RNA was pelleted as described before, except for $15 \mathrm{~h}$ at $85,000 \times \mathrm{xg}$ at $20^{\circ} \mathrm{C}$ in a SW28.1 rotor $(12 \mathrm{ml}$ supernatant on $3.5 \mathrm{ml} 5.7 \mathrm{M} \mathrm{CsCl} / 0.1 \mathrm{M}$ EDTA in $5 \mathrm{SW} 28.1$ tubes). From $2 \mathrm{~g}$ of tissue, $3 \mathrm{mg}$ of total RNA was extracted.

\subsection{Amplification of variable region genes}

Random primed cDNA was prepared with $250 \mu \mathrm{g}$ PBL. RNA, while in a separate reaction $300 \mu \mathrm{g}$ spleen RNA was used as template. RNA was heat denatured for $5 \mathrm{~min}$ at $65^{\circ} \mathrm{C}$ in the presence of $20 \mu \mathrm{g}$ random primer (Promega), subsequently buffer and DTT were added according to the suppliers instructions (Gibco-BRL), as well as $250 \mu \mathrm{M} \mathrm{dNTP} \mathrm{(Pharmacia),} 800$

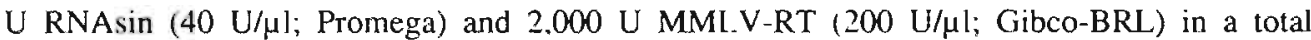
volume of $500 \mu \mathrm{l}$. After $2 \mathrm{~h}$ at $42^{\circ} \mathrm{C}$, the incubation was stopped by a phenol $/$ chloroform extraction; cDNA was precipitated and dissolved in $85 \mu \mathrm{l}$ water.

Oligonucleotides used for PCR amplification of human heavy and light chain V-regions are described in Table I. IgM-derived heavy chain variable regions were obtained by a primary PCR with an lyM constant region primer. All primary PCRs were carried out with separate BACK primers and combined FOR primers, to maintain maximal diversity. The PCR-products were reamplificd with a combination of JHFOR-primers, annealing to the 3 ' end of $\mathrm{VH}$, and Sfitagged VHBACK-primers, annealing to the 5 ' end, and subsequently cloned as VH-fragments. The light chain $V$ genes of the kappa and lambda families were obtained by PCR with a set of CKFOR- or C $\lambda F O R$-primer annealing to the $3^{\prime}$ end of the constant domain and BACK-primers, priming at the 5 ' end of the $V$-regions. The DNA-segments were reamplified with primers tagged with restriction sites and cloned as $\mathrm{V} \kappa \mathrm{C} \kappa$ - and $\mathrm{V} \lambda \mathrm{C} \lambda$-fragments. 
Table I. Oligonucleotide primers used for construction of the library

$\begin{array}{ll}\text { A. Primary amplification } & \text { B. Secundary amplification }\end{array}$

\section{IgM CH1 region}

HUIgMFOR

5. -TGG AAG NGO CAC GTT CTT TTC TTT-3.

$\kappa$ chain constant region

HUCKEOR.

5'-ACA CTC TCC CCT GTT GAA GCT CTT-3

$\lambda$ chain constant region

HuC $\boldsymbol{\lambda} 2$-FOR

$\mathrm{HuC \lambda} 7-\mathrm{FOR}$

5. -TGA ACA TTC TGT AGG GGC CAC TG-3

5.-AGA GCA TTC TGC AGO GGC CAC TG-3.

\section{$V_{H}$ back}

HUVH1B/7A-BACK

HUVH1C-BACK

HUVH $2 \mathrm{~B}-\mathrm{BACK}$

HUVH 3 B-BACK

HUVH $3 \mathrm{C}-\mathrm{BACK}$

HUVH4B-BACK

HUVH $4 \mathrm{C}-\mathrm{BACK}$

HUVH5B-BACK

HUVH 6 A-BACK
B. Secundary amplification $\kappa$ chain constant region

HUCK-FOR-ASC

\section{$\lambda$ chain constant region}

HUC $\lambda 2-F O R-A S C$

HuCd7-FOR-ASC

\section{$V_{H}$ back}

HUVH1B/7A-BACK-SFI

HUVHIC-BACK-SFI

HUVH2B-BACK-SPI

HUVH 3 B-BACK-SFI

HUVH 3 C-BACK-SF 1

HUVH4B-BACK-SFI

HUVH $4 \mathrm{C}-\mathrm{BACK}-\mathrm{SFI}$

HUVH5B-BACK-SFI

HUVH 6A-BACK-SFI
5'-ACC GCC TCC ACC GGG CGC GCC TTA TT'A ACA CTC TCC CCT GTT GAĀ GCT CTY'-3

5.-ACC GCC TCC ACC GGG CGC GCC TTA TTA TGA ACA TTC TGT AGG GGC CAC TG-3

5'-ACC GCC TCE ACC GGC CGC GCC TTA TTA AGA GCA TTC TGC AGG. GGC CAC TG-3.

\section{$\mathrm{VH}$ forward}

HUJHI/2-FOR

HUJH3-FOR

HUJH4/5-FOR.

HUJH $6-$ FOR
5. -GTC CTC GCA ACT GCG GCC CAG CCG GCC ATG GCC CAG RTG CAG CTG GTG CAR TCT GG -3. 5*-GTC CTC GCA ACT GCG GCC CAG CCG GCC ATG GCC SAG GTC CAG CTG GTR CAG TCI GG-3

5:-GTC CTC GCA ACT GCG GCC CAG CCG GCC ATC GCC CAG RTC ACC TTG AAG GAG TCT GG-3.

5.-GTC CTC GCA ACT GCG GCC CAG CCG GCC ATG GCC SAG GTG CAG CTG GTG GAG TCT GG-3

5.-GTC CTC GCA ACT GCG OCC CAG CCG GCC ATG GCC GAG GTG CAG CTG GTG GAG WCY GC-3

5.-GTC CTC GCA ACT GCG GCC CAG CCG GCC ATG GCC CAG GTG CAG CTA CAG CAG TGG GG-3.

5.-GTC CTC GCA ACT GCG GCC CAG CCG GCC ATG GCC CAG STG CAG CTG CAG GAG TCS GG-3

5'-GTC CTC GCA ACT GCG GCC CAG CCG GCC ATG GCC GAR GTG CAG CTG GTG CAG TCT' GG-3.

5.-GTC CTC GCA ACT GCG GCC CAG CCG GCC ATG GCC CAG GTA CAG CTG CAG CAG TCA GG-3.
1-TGGA GGA GAC GGT GAC CAG GGT GCC-3.

5. TGA AGA GAC GGT GAC CAT TGT CCC-3.

5 - TGA GGA GAC GGT GAC CAG GGT TCC-3

$5^{-}$-TGA GGA GAC GGT GAC CGT GGT CCC-3. 
Table I. (continued)

\section{A. Primary amplification}

\section{VK back}

HUVK1B-BACK

HUVK2-BACK

HUVK $3 B-B A C K$

HUVK4B-BACK

HLVK5-BACK

HUVK6-BACK

\section{$\mathrm{V} \lambda$ back}

HUVIA-BACK

HUVגIB-BACK

HUVג1C-BACK

HUVג.2-BACK.

HuV 3 A $-\mathrm{BACK}$

HuV $33 \mathrm{~B}-\mathrm{BACK}$

HUV 4 -BACK

HuVA 5-BACK

HuV $6-B A C K$

HuV $77 / 8-B A C K$

HUV 9 -BACK

\section{B. Secundary amplification}

\section{$V_{K}$ back}

5"-GAC ATC CAG WTG ACC CAG TCT CC-3

5:-GAT GTT GTG ATG ACT CAG TCT CC-3

5.-GAA ATT GTG WTG ACR CAG TCT CC-3

5:-GAT ATT GTG ATC ACC CAC ACT CC-3'

5 -GAA ACG ACA CTC ACG CAG TCT CC-3.

5'-GAA ATT GTG CTG hCT CAG TCT CC-3

HUVKZ-BACK-APA

$H u V K 3 B-B A C K-A P Z$

HUVKAB-BACK-APA

HUVK5-BACR - APA

HUVK6-BACK-APA

\section{$\mathrm{V} \lambda$ back}

5.-CAG TCT GTG CTO ACT CAG CCA CC-3

5.-CAG TCT GTG YTG ACG CAG CCCG CC-3

$5^{\text {- }}$-CAG TCT GTC GTG ACG CAG CCG CC-3.

5. - CAR tct GCC CTG act CAG CCT-3.

5:-TCC TAT GWG CTG ACT CAG CCA CC-3

5*-TCT TCT GAG CTO ACT CAG GAC CC-3

5-CAC GTY ATA CTG ACT CAA CCG $\mathrm{CC}^{-3}$

5:-CAG GCT GTG CTG ACT CAG CCG TC-3

5'-AAT TTT ATG CTG ACT CAG CCC $\mathrm{CA}-3$

5.-CAG RCT GTG GTG ACY CAG GAG CC-3.

HUV 1 IA-BACK-APA

HuV入.1B-BACK-APA

HUVA1C-BACK-APA

HUV $\lambda Z-B A C K-A P A$

HUVA $3 A-B A C K-A P A$

HuV $2.3 \mathrm{~B}-\mathrm{BACK}-\mathrm{APF}$

HUVDA - BACK - APA

HuV $25-$ BACK-APA

HuV $26-B A C K-A P A$

HuV $\lambda 7 / 8-B A C K-A P A$

HuV $29-B A C K-A P A$
$5^{*}-$ ACC GCC TCC ACC AGS GCA CTT GAC ATC CAG WTG ACC CAG TCT CC-3 5:-ACC GCC TCC ACC AGT GCA CTT GAT GTT GTG ATG ACT CAG TCT CC-3

5--ACC GCC NCC ACC AGT GCA CTT GAA ATT GTG WTG ACR CAG TCT CC-3.

5:-ACC GCC TCC ACC AGT GCA CTT GAT ATT GTG ATG ACC CAC ACT CC-3

5. - ACC GCC TCC ACC AGT GCA CTT GAA ACG ACA CTC ACG CAG TCT CC-3.

5.-ACC GCC TCC ACC AGT GCA CTT GAA ATT GTG CTG ACT CAG TCT CC-3

5:-ACC GCC TCC ACC AGT GCA CAG TCT GTG CTG ACT CAG CCA CC-3.

5.-ACC GCC TCC ACC AGT GCA CAG TCT GTG YTT ACG CAG CCG CC-3.

5'-ACC GCC TCC ACC AGT GCA CAG TCT GTC GTG ACG CAG CCG CC-3

5. -ACC GCC TCC ACC AGT GCA CAR TCT GCC CTG ACT CAG CCT-3

5.-ACC GCC TCC ACC AGT GCA CDT TCC TAT GWG CIG ACT CAG CCA CC-3

5. -ACC GCC TCC ACC AGT GCA CTT TCT TCT GAG CTG ACT CAG GAC CC-3.

$5 * A C C$ GCC TCC ACC AGT GCA CAC GTT ATA CTG ACT CAA CCG CC-3"

5:-ACC GCC TCC ACC AGT GCA CAG GCT GTG CTG ACT CAG CCG TC-3:

5. $=$ ACC GCC TCC ACC AGT GCA CTT AAT 'TTT ATG CTG ACT CAG CCC CA-3.

5.-ACC GCC TCC ACC AGT GCA CAG RCT GTG GTG ACY CAG GAG CC-3:

5.-ACC GCC TCC ACC AGT GCA CWG CCT GTG CIG ACT CAG CCM CC-3.TCT CC-3 
PCR was performed in a volume of $50 \mu \mathrm{l}$ using AmpliTaq polymerase (Cetus) and $500 \mathrm{pM}$ of each primer for 28 cycles $\left(1 \mathrm{~min}\right.$ at $94^{\circ} \mathrm{C}, 1 \mathrm{~min}$ at $55^{\circ} \mathrm{C}$ and $2 \mathrm{~min}$ at $72^{\circ} \mathrm{C}$ ), 9 separate $\mathrm{IgM}$ derived $\mathrm{VH}$-amplifications were generated with $2 \mu \mathrm{l}$ random primed cDNA (equivalent to $6 \mu \mathrm{g}$ PBL RNA or to $7 \mu \mathrm{g}$ spleen RNA) as template for each reaction. For the light chain families, 6

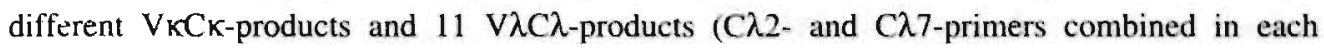
reaction) were obtained. All products were purified from agarose gel with the QIAex-II extraction kit (Qiagen). As input for reamplification to introduce restriction sites, 100-200 ng purified DNA-fragment was used as template in a $100 \mu \mathrm{l}$ reaction volume. The large amount of input, ensuring the maintenance of variability, was checked by analysis of $4 \mu \mathrm{l}$ of the "unamplified" PCR-mixture on agarose gel.

\subsection{Construction of the primary and secondary repertoires}

For the construction of the primary heavy chain and the two primary light chain repertoires, the PCR-products, appended with restriction sites, were gel purified prior to digestion and the different $\mathrm{VH}$-, $\mathrm{V} \kappa_{\kappa}$ - and $\mathrm{V} \lambda$-families combined into three groups. The $\mathrm{V} \kappa \mathrm{C} \kappa-$ and $\mathrm{V} \lambda \mathrm{C} \lambda$ fragments were digested with $A p a L I$ and $A s c l$, and cloned into the phagemid vector pCESI (Fig. 1). The VH-fragments, $1.5 \mu \mathrm{g}$ in total, were digested with SfiI and $B s t$ EII and ligated in a 100 $200 \mu \mathrm{l}$ reaction mixture with $9 \mathrm{U}$ T4-DNA ligase at room temperature to $4 \mu \mathrm{g}$, gel-purified vector pUC119-CESI (similar to vector pCESI, but with the pIII gene deleted). The desalted ligation mixture for light or heavy chain pools was used for electroporation of the $E$. coli strain TG1, to create the one-chain libraries.

The Fab library was obtained by cloning of VH fragments, digested from plasmid-DNA prepared from the heavy chain repertoires, into the plasmid collection containing the light chain repertoires. Plasmid DNA isolated from at least $3 \times 10^{9}$ bacteria of the VH library was digested with $S f i$ and $B s t$ EII for cloning in the vector that already contained $\kappa$ and $\lambda$ light chain libraries. To retain clones with internal $B s t E I I$ site in the $V \lambda$ (this site is relatively frequent in some $\lambda$ germline $V$-segments (22), and also in the constant domain of one of the $\lambda$ families), the cloning of VHCHI in the $\lambda$ light chain repertoire containing vector was also carried out using SfiI and NotI cloning sites, to create a less restriction-biased $V \lambda$ libary.

\subsection{Selection of the library}

The rescue of phagemid particles with helper phage M13-KO7 was performed according to (18) on 10-L scale, using representative numbers of bacteria from the library for inoculation, to ensure the presence of at least 10 bacteria from each clone in the start inoculum. For selections, $10^{13}$ cfu's (colony forming units) were used with antigens immobilised in immunotubes (Maxisorp tubes, Nunc) (18) or with soluble biotinylated antigens (23). The amount of the immobilised antigens tetanus toxoid and the hapten phenyl-oxazolone (conjugated to BSA in a ratio of 17 to 1) was reduced 10 -fold during subsequent selection rounds, starting at $100 \mu \mathrm{g} / \mathrm{ml}$ at 
round 1. Capture with biotinylated antigen in solution was used for a 100 -mer peptide encoding five copies of the tandem repeat of MUCI (24), or with human Chorionic Gonadotropin (hCG), human Luteinizing Hormone (hLH), human Follicle Stimulating Hormone (hFSH) and its chimeric derivative (hFSH-CTP, containing the carboxy terminal peptide from the hCG $\beta$ subunit fused to the $\beta$-subunit of hFSH). Antigens were biotinylated at a ratio of ten to twenty molecules NHS-Biotin (Pierce) per molecule antigen according to the suppliers recommendations. Unless stated otherwise, the antigens were used for selection at concentrations of $100 \mathrm{nM} .30 \mathrm{nM}$ and $10 \mathrm{nM}$ during round 1,2 and 3 respectively. For hFSH-CTP 50, 15 and $10 \mathrm{nM}$ was used respectively; for $\mathrm{MUCl}$ peptide, $500,100,20$ and $5 \mathrm{nM}$ was used.

\subsection{Screening and sequencing of clones}

Soluble Fab was produced from individual clones as described before (18). Culture supernatants were tested in ELISA with directly coated antigen or indirectly captured biotinylated antigen via immobilised biotinylated BSA-streptavidin. Tetanus toxoid and phOxBSA were coated at $10 \mu \mathrm{g} / \mathrm{ml}$ in $0.1 \mathrm{M} \mathrm{NaHCO}_{3} \mathrm{pH} 9.6$ for $16 \mathrm{~h}$ at $4^{\circ} \mathrm{C}$. For coating of hCG and hFSH-CTP a concentration of $4 \mu \mathrm{g} / \mathrm{ml}$ in $50 \mathrm{mM} \mathrm{NaHCO} 3 \mathrm{pH} 9.6$ was used. For capture of biotinylated antigens, biotinylated BSA was coated at $2 \mu \mathrm{g} / \mathrm{ml}$ in PBS during $1 \mathrm{~h}$ at $37^{\circ} \mathrm{C}$. After 3 washes with PBS-0.1\% (v/v) Tween 20 (PBST), plates were incubated during $1 \mathrm{~h}$ with streptavidin (10 $\mu \mathrm{g} / \mathrm{ml}$ in PBS / 0.5\% gelatin) (24). Following washing as above, biotinylated antigen was added for an overnight incubation at $4^{\circ} \mathrm{C}$ at a concentration of $0.5 \mu \mathrm{g} / \mathrm{ml}$ for MUC1 peptide, $3 \mu \mathrm{g} / \mathrm{ml}$ for $\mathrm{hLH}$, and $0.6 \mu \mathrm{g} / \mathrm{ml}$ for hFSH (binding to hCG was tested with directly coated antigen). The plates were blocked during $30 \mathrm{~min}$ at room temperature with $2 \%(w / v)$ semi-skimmed milk powder (Marvel) in PBS. The culture supernatant was diluted 1 or 5 -fold in $2 \%(w / v)$ Marvel / PBS and incubated 2 h; bound Fab was detected with anti-myc antibody $9 \mathrm{E} 10$ (5 $\mu \mathrm{g} / \mathrm{ml}$ ) recognising the myc-peptide tag at the carboxyterminus of the heavy Fd chain, and rabbit anti-mouse-HRP conjugate (DAKO) (18). Following the last incubation, staining was performed with tetramethylbenzidine (TMB) and $\mathrm{H}_{2} \mathrm{O}_{2}$ as substrate and stopped by adding half a volume of $2 \mathrm{~N} \mathrm{H}_{2} \mathrm{SO}_{4}$; the optical density was measured at $450 \mathrm{~nm}$. Clones giving a positive signal in ELISA (over $2 \mathrm{x}$ the background), were analysed by $B s t$ NI-fingerprinting of the PCRproducts obtained by amplification with the oligonucleotide primers M13-reverse and geneIIIforward (18).

Large-scale induction of soluble Fab fragments from individual clones was performed on 50 $\mathrm{ml}$ scale in $2 \times \mathrm{TY}$ containing $100 \mu \mathrm{g} / \mathrm{ml}$ ampicillin and $2 \%$ glucose. After growth at $37^{\circ} \mathrm{C}$ to an $\mathrm{OD}_{600}$ of 0.9 , the cells were pelleted $(10 \mathrm{~min}$ at $2,934 \mathrm{xg}$ ) and resuspended in $2 \times \mathrm{TY}$ with ampicillin and $1 \mathrm{mM}$ IPTG. Bacteria were harvested after $3.5 \mathrm{~h}$ growing at $30^{\circ} \mathrm{C}$ by centrifugation (as before); periplasmic fractions were prepared by resuspending the cell pellet in $1 \mathrm{ml}$ ice cold PBS. After 2 to $16 \mathrm{~h}$ rotating head-over-head at $4^{\circ} \mathrm{C}$, the spheroplasts were removed by two centrifugation steps: after spinning during $10 \mathrm{~min}$ at $3,400 \mathrm{xg}$, the supernatant 
was clarified by an additional centrifugation step during $10 \mathrm{~min}$ at $13,000 \mathrm{xg}$ in an eppendorf centrifuge. The periplasmic fraction obtained was directly used for determination of fine specificities by surface plasmon resonance or for western blot studies (described below).

For sequencing, plasmid DNA was prepared from $50 \mathrm{ml}$ cultures grown at $30^{\circ} \mathrm{C}$ in LBmedium, containing $100 \mu \mathrm{g} / \mathrm{ml}$ ampicillin and $2 \%$ glucose, using the QIAGEN midi-kit (Qiagen). Sequencing was performed with the thermocycling kit (Amersham) with CY5-labeled primers CHIFOR (5'-GTC CTT GAC CAG GCA GCC CAG GGC-3') and M13REV (5'-CAG GAA ACA GCT ATG AC-3'); samples were run on the ALF-Express (Pharmacia). V-gene sequences were aligned to V-base (Tomlinson et. al., V-BASE, MRC Centre for Protein Engineering, 1997, http://www.mrc-cpe.cam.ac.uk/imt-doc/public/INTRO.html) or to the Sanger Centre database (Sanger Centre Germline Query, 1997, http//www.sanger.ac.uk/Data Search/gqsearch.html).

\subsection{Determination of fine specificities of the anti-hormone Fab's by weste'rn blot and surfuic plasmon resonance}

An hCG-preparation purified from urine and immuno-atifinity purified recombinant $h L H$, hFSH and hFSH-CTP produced in CHO-cells $(25,26)$ were used for western blot studies as was described (27). Between 0.5 and $1 \mu \mathrm{g}$ of each hormone was loaded per lane; proteins werc diluted in non-reducing sample buffer and boiled during $5 \mathrm{~min}$ or directly applied on gel without heat-treatment; proteins were transferred to blotting membrane by electrotransfer. Blots were subsequently incubated for $16 \mathrm{~h}$ at room temperature with a 10-fold diluted periplasmic fraction in PBS / 4\% Marvel. Bound Fab was detected with anti-myc antibody $9 \mathrm{E} 10(5 \mu \mathrm{g} / \mathrm{ml})$ and 4,000fold diluted anti-mouse alkaline phosphatase-conjugate (Promega), using the substrates 5-bromo1-chloro-3-indolyl phosphate (BCIP) and nitro blue tetrazolium (NBT) (Boehringer Mannheim) for visualisation.

The specificity of the Fab's was further characterised by surface plasmon resonance (BIAcore 2000, Biacore). Recombinant hLH, hFSH and the urinary hCG were immobilised on the flow-cells of a CM-chip using the NHS/EDC-kit (Pharmacia), yielding a surface of $1906 \mathrm{RU}$ for hLH, 1529 RU for hFSH and 1375 RU for hCG. Periplasmic fractions were diluted three-fold in Hepes Buffered Saline (HBS; $10 \mathrm{mM}$ Hepes, $3.4 \mathrm{mM}$ EDTA, $150 \mathrm{mM} \mathrm{NaCl}, 0.05 \%(\mathrm{v} / \mathrm{v})$ surfactant $\mathrm{P} 20, \mathrm{pH} 7.4$ ) and analysed using a flow rate of $10 \mu 1 / \mathrm{min}$.

\subsection{Purification of soluble Fab fragments}

Fab's were obtained by refolding of the total bacterial proteins from a $50 \mathrm{ml}$ culture (28). Briefly, the pelleted cells from a $50 \mathrm{ml}$ induced bacterial culture were resuspensed in $8 \mathrm{ml} 8 \mathrm{M}$ urea (in PBS). After sonication, the mixture was rotated head over head for 30 min and insoluble material was removed by centrifugation for $30 \mathrm{~min}$ at $13,000 \mathrm{xg}$. The supernatant was dialysed against PBS with four buffer changes. Insoluble proteins were removed by centrifugation and the 
How through fraction. obtained by filtration through a $0.2 \mu \mathrm{m}$ membrane, was immediately loaded on an $\mathrm{hC}(\mathrm{i}$ column (hed volume $0.3 \mathrm{ml}$ ). The column material was prepared by coupling $8.4 \mathrm{mg}$ protein to one gram Tresyl sepharose according to the suppliers instructions (Pierce). The column ( $1 \mathrm{ml}$ column material) was washed with 10 volumes $100 \mathrm{mM}$ Tris, $500 \mathrm{mM} \mathrm{NaCl} \mathrm{pH}$ 7.5. subsequently with 10 volumes $100 \mathrm{mM}$ Tris $/ 500 \mathrm{mM} \mathrm{NaCl} \mathrm{pH} 9.5$ and with 2 volumes $0.9 \% \mathrm{NaCl}$, bound $\mathrm{Fab}$ was eluted with two volumes $0.1 \mathrm{M} \mathrm{TEA}$ and immediately neutralised with 0.5 volume $1 \mathrm{M}$ Tris $\mathrm{pH}$ 7.5. The Fab fraction was dialysed against PBS using a Microcon 30 spin dialysis lilter (Amicon). Finally, a gel-filtration analysis was carried out on a Superdex 75 HR column (Pharmacia). The yicld was determined by measuring the optical density at 280 $\mathrm{nm}$ (using a molar extinction coefficient of 13 for Fab’s).

3.8 Determination of on- and off-rate using surface plasmon resonance with crude Fab preparations

The kinetics of binding were analysed by surface plasmon resonance on three different hCG surfaces (303 RU, 615 RU and 767 RU immobilised, with 4955 RU BSA on a separate flow cell as a negative control). Fab present in crude periplasmic extracts was quantified on a high density surface of purified anti-human-Fab polyclonal antibody (Pierce) as described (29). Anti-hCG Fab's controls were purified by affinity chromatography on hCG columns as described above and used to calibrate the system.

\section{Results}

\subsection{Design of the naïve phage antibody library}

We considered a number of variables to address in the construction of a novel, very large antibody phage library: (i) the primer design was optimised for amplification of variable gene pools to maintain maximum diversity; (ii) a highly efficient two-step cloning method was developed to obtain a very large naïve library: (iii) an antibody format and compatible cloning vector were chosen, which should permit the rapid down-stream analysis of selected clones.

In order to achieve access to as many different human heavy and light chain V-region gene segments as possible, a new set of oligonucleotide primers was developed (Table I), the design of which was based on the most recent sequence information provided by the V-base (see Materials and Methods). The primers should allow efficient amplification of all commonly used $\mathrm{V}$-gene segments. Further, to obtain large sized libraries (over $10^{10}$ in diversity), we used a twostep cloning procedure: heavy and light chain variable genes were first separately cloned as digested PCR products, and were then combined by restriction fragment cloning to form a large library of Fab fragments. This cloning procedure should be a more efficient route for library 
construction than the relatively inefficient direct cloning of digested PCR-products, while avoiding the DNA instability often associated with in vivo recombination systems (30).

As choice of antibody format, we preferred the Fab above the scFv format, because this would allow us to develop rapid high through-put affinity-screening assays for crude antibody preparations. Many scFv's indeed form higher molecular weight species including dimers $(31,32)$ and trimers (33), which complicate both selection and characterisation. We chose the Fab display format in which the heavy chain is linked to the phage coat protein pIII, and also carries a tag for detection and purification (see below). The light chain is expressed as separate fragment secreted into the periplasm, where it can pair with the heavy chain (34).

Fig. 1. Schematic representation (A) and polylinker region (B) of the phagemid vector pCES1.

A.

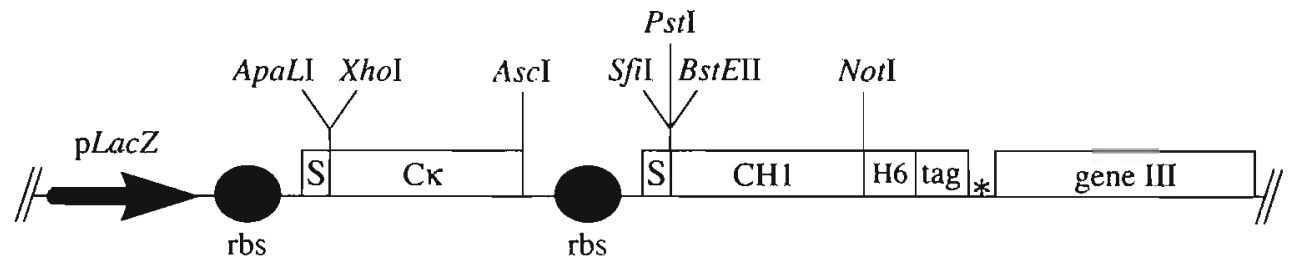

B.

Signal sequence ApaLI +I

-. TTA TTC GCA. ATT CCT TTA GTT GTT CCT TTC TAT TCT CAC AGT GCA CAG GTC CAA CTG CAG GTC GAC CTC: GAG

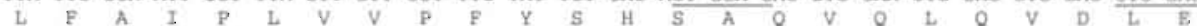

AscI $\quad r$ bS

ATC AAA CGT GGA ACT GTG -.. GGA GAO TGT TAA TAA GGC GCG CCA, ATT CTA TTT CAA GGA GAC AGT CAT A

I K R G T V V G E C *

Human CK gene Stops

Signal sequence

ATG AAA TAC CTA TTG CCT ACG GCA GCC GCT GGA TTG TTA TTA CTC GCG GCC CAG CCG GCC ATG GCC CAG GTG

M K $\mathrm{K}$ Y L L F

PstI BsteII NotI

CAG CTG CAG GAG AGC GGG GTC ACC GTC TCA AGC GCC TCC ACC -.. AAA TCT TGT GCG GCC GCA CAT CAT CAT CAT

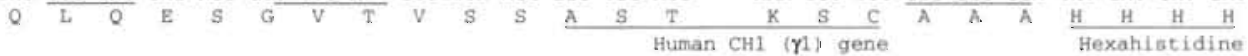

CAT CAC GGG GCC GCA GAA CAA AAA CTC ATC TCA GAA GAG GAT. CTG AAT GGG GCC GCA TAG. ACT GTT N..

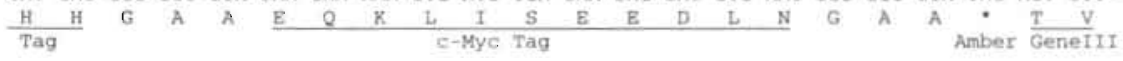

To incorporate all these improvements, a new phagemid vector, pCES1 (Fig. 1), was constructed, that allows the stepwise cloning of antibody fragments in Fab format. In this vector system, the variable heavy chain region genes are cloned as VH-gene fragments; the vector 
supplies all Fab's with a human gamma-1 CHI gene. The Fd fragment is fused to two tags for purification and detection (a histidine tail for Immobilised Metal Affinity Chromatography (3.5) and a $c$-myc-derived tag (36)), followed by an amber stop codon (34) and the minor coat protein III of filamentous phage fd. The antibody light chain is cloned as full VLCL fragment, for directed secretion and assembly with the $\mathrm{VHCHI}$ on the phage particle.

\subsection{Library construction}

The Fab library was constructed in two-steps. In the first step, variable region gene pools were amplified from approx. $4 \times 10^{8}$ B-cells from the PBLs of four healthy donors, and, as a source of possibly more heavily mutated IgM antibodies, from a segment of a (tumor-free) spleen removed from a patient with gastric carcinoma, containing approximately $1.5 \times 10^{8} \mathrm{~B}$-cells (37). Only IgM-derived VH segments were amplified by using an amplification with an oligonucleotide primer located in the first constant domain of this isotype. These products were cloned into phagemid vector pCESl for VL, and in pUC119-CES1 for VH (cloning was more efficiently in the smaller sized vector, in which gene III was deleted). The PBL and spleen derived VH, VK and V $\lambda$-libraries were cloned separately to maintain diversity, to yield one-chain libraries in size typical for libraries made by cloning of PCR-fragments (18): $1.75 \times 10^{8}$ individual clones for the heavy chain, $9.4 \times 10^{7}$ clones for $V K$. and $5.2 \times 10^{7}$ clones for $V \lambda$. An overview is given in Table II.

Table II. Size and composition of the phage antibody libraries

\begin{tabular}{|c|c|c|c|c|}
\hline & $\begin{array}{l}\text { Source } \\
\text { B-cells }\end{array}$ & Library & $\%$ insert & $\begin{array}{l}\text { Size } \\
\text { (cfu) }\end{array}$ \\
\hline \multirow{7}{*}{$\begin{array}{l}\text { one-chain } \\
\text { libraries }\end{array}$} & \multirow{3}{*}{ PBL } & $\mathrm{VH}$ & 64 & $1.8 \times 10^{8}$ \\
\hline & & $V K$ & 100 & $5.7 \times 10^{7}$ \\
\hline & & $v \lambda$ & 96 & $4.5 \times 10^{7}$ \\
\hline & \multirow{4}{*}{ spleen } & & & \\
\hline & & VH & 75 & $8.0 \times 10^{7}$ \\
\hline & & $V_{K}$ & 100 & $3.0 \times 10^{7}$ \\
\hline & & $v \lambda$ & 80 & $1.0 \times 10^{7}$ \\
\hline \multirow[t]{2}{*}{ Fab-library } & PBL & & 90 & $1.95 \times 10^{10}$ \\
\hline & spleen & & 80 & $2.35 \times 10^{10}$ \\
\hline total size & & & 86 & $4.3 \times 10^{10}$ \\
\hline
\end{tabular}

\footnotetext{
${ }^{a}$ Not corrected for clones without Fab encoding insert.
} 
In the second step, the heavy chain fragments were digested from plasmid DNA isolated from the primary VH repertoire, and cloned into the vector containing the light chain repertoires (again separately for PBL and spleen derived repertoire; Table II). The libraries were combined using this efficient cloning procedure, to create a naïve Fab repertoire with $3.7 \times 10^{10}$ individual clones $\left(4.3 \times 10^{10}\right.$ recombinant clones, $86 \%$ of which have a full-length Fab insert), with $70 \%$ of clones harbouring a kappa light chain, $30 \%$ a lambda chain. All of 20 clones with full length Fab insert tested scored positive in dot-blot analysis with the $9 \mathrm{E} 10$ antibody to indicating an expression level of soluble Fab of at least $0.2 \mathrm{mg} / \mathrm{L}$.

\subsection{Quality control of the library by selection with a panel of antigens}

We evaluated the library by selection with different antigens, the screening data of which are summarised in Table III. First, the results from three model antigens, the protein tetanus toxoid, the hapten 2-phenyloxazol-5-one (phOx) (38) and the peptide MUCl, are discussed. Three rounds of biopanning on tetanus toxoid yielded a diverse set of ELISA positive Fab's: in a series of 47 tetanus toxoid binding Fab's, at least 21 were different with regard to BstNIfingerprint. Similarly, an extensive panel of phOx-specific Fab's was retrieved after three rounds of panning: at least 24 different clones were identified in a series of 50 ELISA positive clones. Solution capture with biotinylated $\mathrm{MUCl}$ peptide resulted in the selection of 14 different antibody fragments out of 37 ELISA-positive clones selected after 3 rounds ('Table III).

Table IJI. Overview of resulis of selections on a chiverse sel of antigens.

\begin{tabular}{ccccc}
\hline Antigen & round 2 & $\begin{array}{c}\text { \#ELISA positives } \\
\text { round 3 }\end{array}$ & round 4 & $\begin{array}{c}\text { \# different } \\
\text { Ag-spec. clones }\end{array}$ \\
\hline$\Pi^{\mathrm{a}}$ & $13 / 36$ & $69 / 80$ & - & $>21$ \\
$\mathrm{phOx}^{\mathrm{b}}$ & $29 / 37$ & $70 / 80$ & - & $>24$ \\
$\mathrm{MUC1}^{\mathrm{c}}$ & $1 / 37$ & $32 / 87$ & $25 / 87$ & 14 \\
$\mathrm{hCG}^{\mathrm{d}}$ & - & $24 / 48$ & $34 / 48$ & 8 \\
$\mathrm{hLH}^{\mathrm{e}}$ & - & $40 / 45$ & $30 / 45$ & $>21$ \\
$\mathrm{hFSH}^{\mathrm{r}}$ & - & $30 / 45$ & $40 / 45$ & 6 \\
$\mathrm{hFSH}-\mathrm{CTP}^{\mathrm{g}}$ & - & $16 / 48$ & $17 / 48$ & 7 \\
\hline
\end{tabular}

"TT: tetanus toxoid: ${ }^{b}$ phOx: 2-phenyloxazol-5-one: ' MUCl: Mucin-1 derived peptide: ${ }^{d}$ hCG: human Chorionic Gonadotropin; " hLH: human Luteinizing Hormone; ${ }^{f}$ hFSH: human Follicle Stimulating Hormone: " hFSH-CTP: chimeric product of $\beta$-CTP of hCG fused to $\beta$-FSH.

\subsection{Rapid dissociation rate determination}

With such large panels of antibodies isolated, it is crucial to have methods available to readily determine the kinetic parameters of each individual antibody-antigen interaction. Such an 
assay should be robust and ideally employ non-purified antibody fragments. We tested if it would be feasible to use periplasmic fractions prepared from small scale cultures for a rapid and accurate determination of the off-rate of the antibodies using surface plasmon resonance. An example of an overlay plot with the sensorgrams from a series of tetanus toxoid specific Fab's is shown in Fig. 2. The plot of $\ln \left(\mathrm{R}_{0} / \mathrm{R}\right)$ versus time (Fig. 2. lower graphs) reveals a linear relation with slope $k_{d}$ (off-rate), thereby confirming a monophasic dissociation, which can be expected for a truly monomeric Fab-fragment binding to a low density antigen surface. Using this off-rate screening assay, we determined the off-rates for the best tetanus toxoid and MUC1 specific Fab's to be in the order of $10^{-2}$ to $10^{-4} \mathrm{~s}^{-1}$ (Fig. 2).
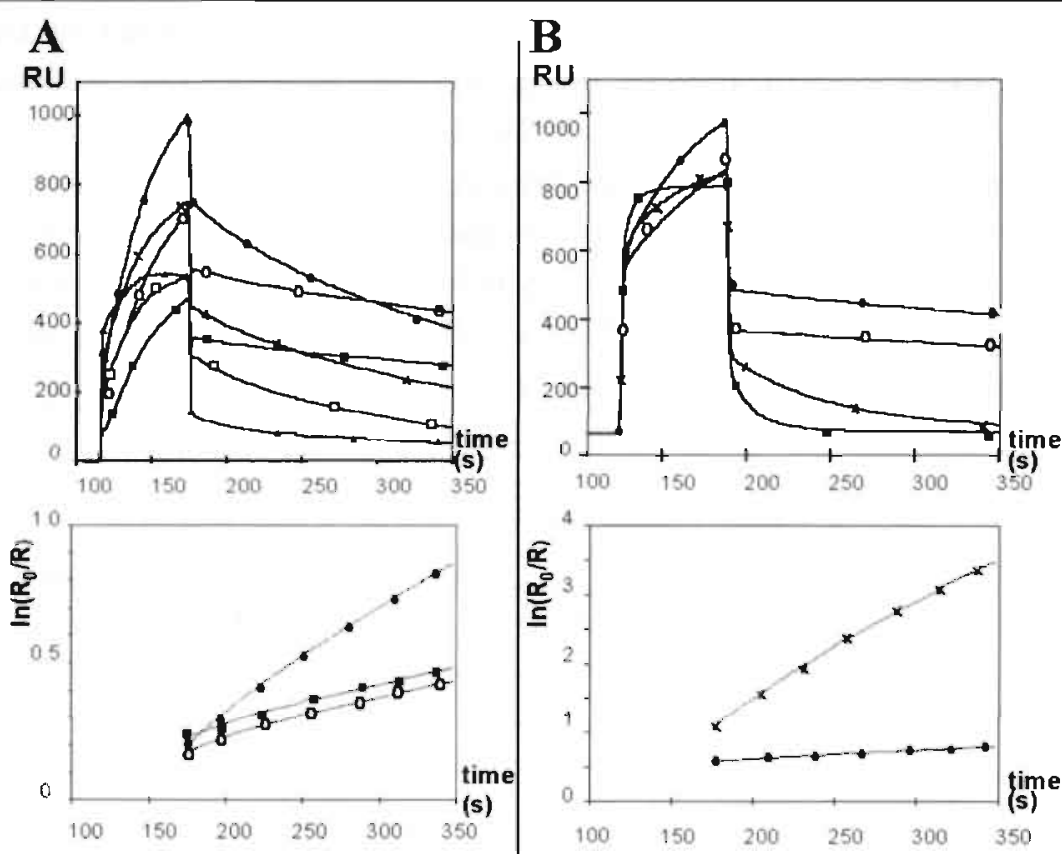

$\begin{array}{ll}\text { Antibody } & \mathbf{k}_{\text {ort }}\left(\mathbf{s}^{-1}\right) \\ \text { Fab 1A o } & 9.45 \times 10^{-4} \\ \text { Fab 1B } & 4.93 \times 10^{3} \\ \text { Fab 3D a } & 7.62 \times 10^{3} \\ \text { Fab 3E } & 8.17 \times 10^{3} \\ \text { Fab 1F } & 4.46 \times 10^{3} \\ \text { Fab 2F } & 1.86 \times 10^{3}\end{array}$

$\begin{array}{ll}\text { Antibody } & \mathbf{k}_{\text {ofr }}\left(\mathbf{s}^{-1}\right) \\ \text { MUC\#4: } & 9.1 \times 10^{-4} \\ \text { MUC\#9 } & 7.6 \times 10^{-3} \\ \text { MUC\#19: } & 3.3 \times 10^{3} \\ \text { MUC\#28: } & 1.9 \times 10^{2}\end{array}$

Figure 2. Off-rate screening in BIAcore of selected Tetanus toxoid and MUCI binding Fab fragments. Periplasmic fractions from 4 anti-tetanus toxoid clones (A) and from anti-MUCl clones (B) were injected at $t=$ 115 s on flow cells with immobilised tetanus toxoid and MUCl peptide, respectively. At $t=175$ s the dissociation phase started by washing with HBS-buffer. Below the sensorgram, the derivative $\ln \left(R_{d} / R\right)$ of two representative clones was plotted against the time $\left(R_{0}\right.$ taken from $\left.t=175 \mathrm{~s}\right)$, which should be linear for a truly monophasic dissociation. Resulting dissociation rates are shown below each series of sensorgrams. 


\subsection{Selection of Fab antibodies against related glycoprotein hormones}

As a more stringent test panel of antigens to assay the performance of the library, we chose to derive antibudies to three structurally related glycoproteins: human Chorionic Gonadotropin (hCG), human Luteinizing Hormone (hLH) and human Follicle Stimulating Hormone (hFSH) (reviewed in (39)). These hormones are heterodimers sharing an identical $\alpha$-chain with 92 amino acid residues, but have $\beta$-subunits of different composition and length. The $\beta$-chain of hCG contains 145 amino acid residues, and the one from hLH only 121 residues, the latter showing $85 \%$ homology to $\beta$-hCG. The $\beta$-chain of hFSH is only 111 amino acids and shares $36 \%$ of the residues with $\mathrm{hCG}$. Antibodies that specifically detect hCG have been used extensively in pregnancy tests (39) and for cancer diagnosis $(40,41)$. A large set of antibodies to these targets would extend the limited number of hormone specific antibodies (especially against hLH), obtained using the hybridoma technology (39). The human origin of the antibodies might be beneficial when using these for imaging or therapy of testicular and bladder cancer $(40,41)$.

Selections were thus performed on biotinylated urinary hCG, recombinant hLH, hFSH and hFSH-CTP (the latter is a chimeric molecule containing the carboxy terminal peptide of $\beta$-hCG fused to the $\beta$-chain of FSH (42)). The highest degree of enrichment in respect to the increase in the number of eluted phage particles in round 3 versus round 1 was found for hCG (10,000-fold), followed by hFSH-CTP (1,000-fold), hFSH (300-fold) and hLH (150-fold). Polyclonal phage of selected populations were tested for binding using sensorchips containing immobilised hormones (43). Polyclonal phage selected with hCG showed binding after rounds two and three of selection to all three proteins, i.e. $\mathrm{hCG}, \mathrm{hLH}$, and $\mathrm{hFSH}$, with the strongest signal visible for hCG (Fig. 3A). Similar analysis of the polyclonal phage populations selected for three rounds on hFSH showed a dominance of hFSH-specific binding, while selections on hFSH-CTP yielded binders to both hFSH and hCG (Fig. 3B). Selections on hLH yielded antibodies reactive with hFSH and hCG (thus most likely anti- $\alpha$-chain antibodies; marked LH(1) in Fig. 3B). When hLH was used at lower concentrations (at $10 \mathrm{nM}$ in round $\mathrm{I}$ and $3 \mathrm{nM}$ during the subsequent selection rounds), a signal was seen with streptavidin only (marked LH(2) in Fig. 3B), due to the selection of streptavidin-specific antibodies. Thus, this polyclonal phage screening provides a rapid test to check the overall quality of the clones in the selected repertoire, and may also be used to guide the choice of the conditions for the next selection round (43).

Figure 3. (Next page) Monitoring of selections with polyclonal phage using surface plasmon resonance. Polyclonal phage populations from round 1,2 and 3 (RI, R2 and R3, respectively) of the selection with hCG, were analysed on flow cells with hCG, hLH and hFSH: at $t=70 \mathrm{~s}, t=400 \mathrm{~s}$ and $t=690 \mathrm{~s}$ phige was injected, ending at $t=120 \mathrm{~s}, t=450 \mathrm{~s}$ and $t=740 \mathrm{~s}$ respectively $(A)$. Analysis of phage from round 3 selected with the antigens $\mathrm{hCG}$. hFSH-CTP. hFSH, and hL.H (the latter was selected with $100 \mathrm{nM}$ hormone (coded LH(1)) or $10 \mathrm{nM}$ (coded LH(2)) at round 1), using flow-cells with hCG and streptavidin (upper sensorgran) or with hFSH-CTP, and hCG (lower sensorgram): injection was started at $t=60 \mathrm{~s}, t=270 \mathrm{~s}, t=500 \mathrm{~s}, t=720 \mathrm{~s}$, and $t=950 \mathrm{~s}$, and terminated at $t=120 \mathrm{~s}$, $t=330 \mathrm{~s}, \mathrm{t}=560 \mathrm{~s}, \mathrm{t}=780 \mathrm{~s}$. and $\mathrm{t}=1010 \mathrm{~s}$ respectively $(B)$. 
A
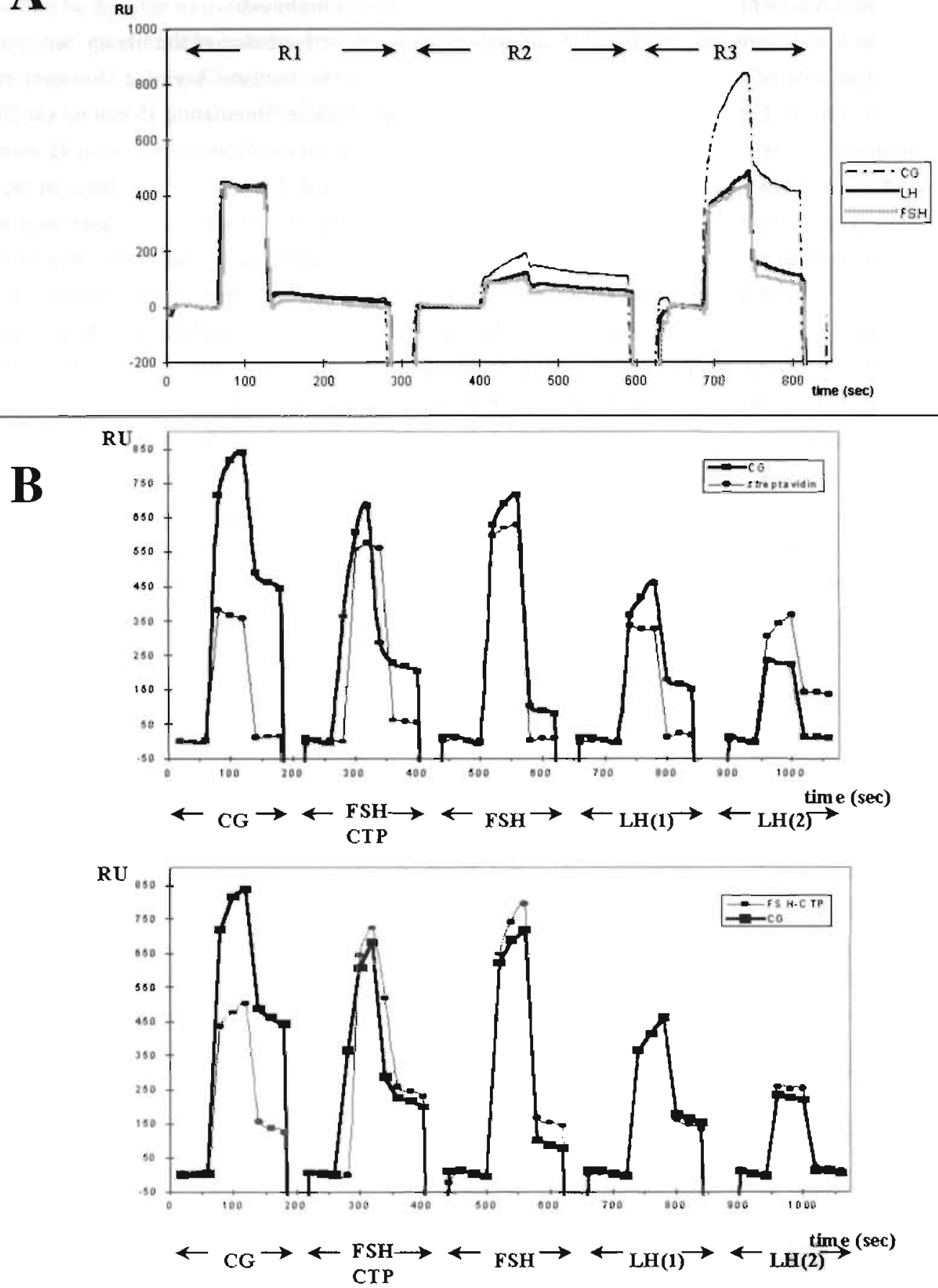


\subsection{Specificity analysis of the selected monoclonal Fab's}

ELISA of monoclonal phage antibodies revealed that three rounds of selection with hCG indeed resulted in the isolation of a high percentage $(74 \%)$ of clones positive for the gonadotropin. $27 \%$ of these clones were hLH cross-reactive; none were reactive against streptavidin. BstNI-fingerprint analysis of the ELISA-positive clones revealed a high degree of diversity ( 8 different patterns). From a representative hCG-specific (coded CG\#4F) and hL.H cross-reactive (CG\#5C) clone, the speciticity was tested in BIAcore using unpurified soluble Fab fragments (Fig. 4). Clone CG\#4F gave a high response on hCG, with no visible binding to either hLH or hFSH-CTP. In contrast, clone CG\#5C bound to hCG and hLH, but not to hFSH-CTP. Western blots, with the different hormones in non-reduced form, showed the specific recognition of the $\beta$-subunit of hCG by clone CG\#4F, while the cross-reactive clone CG\#5C reacted with the $\beta$-subunit of both hCG and hLH (Fig. 5).

Selection with the hormone hLH resulted in the isolation of hLH-specific and hCG crossreactive clones. Examination of individual clones from selection round three in ELISA revealed a large fraction of hLH specific clones (69\%), and a minor group of cross-reactive clones (16\%); no streptavidin reactive clones were selected. Within the group of specific clones, a large array of different species $(>21)$ could be discriminated with fingerprint analysis; however, all crossreactive species had a single pattern. The unique hLH specificity was confirmed for representative clones $\mathrm{LH} \# 2 \mathrm{H}$ and $\mathrm{LH} \# 3 \mathrm{G}$, shown in surface plasmon resonance (shown for clone LH\#3G in Fig. 4); and on western blot (illustrated for clone LH\#3G in Fig. 5). This Fab only recognises the intact $\alpha / \beta$-heterodimer of hLH. Two representative clones of a pan-reactive antibody in ELISA, coded LH\#1C and LH\#3F, reacted in BIAcore with hFSH-CTP, hCG and hLH (shown for clone LH\#3F in Fig. 4), and in western blot analysis with the $\alpha$-chains from all three hormones (not shown).

When hFSH was used as antigen during selection, 6 different antibodies were isolated from the library, with one type, represented by clone FS\#8B, dominating the selected population. This Fab only recognised hFSH in BIAcore (Fig. 4), and, as westem blot analysis demonstrated, in particular its $\beta$-unit (Fig. 5). Further, the specificity of an $\alpha$-chain binding clone, SC\#2B, was confirmed in BLAcore (Fig. 4) and western blot (Fig. 5).

Upon selection with FSH-CTP 7 different $\alpha$-chain specific Fab's were identified by fingerprint analysis, from which the clones coded SC\#2B, SC\#2F, SC\#2G and SC\#4G were examined in more detail. Immunoblot analysis with the recombinant Fab as detecting antibody confirmed the $\alpha$-chain specificity (blot incubated with clone SC\#2B is shown in Fig. 5).

\subsection{Phage selected antigen-specific clones are intact Fub fragments}

There have been some reports on the isolation from scFv or Fab libraries of antigen-specific single-domain or other artificial antibody fragments $(44,45)$. Therefore, we tested the integrity of 

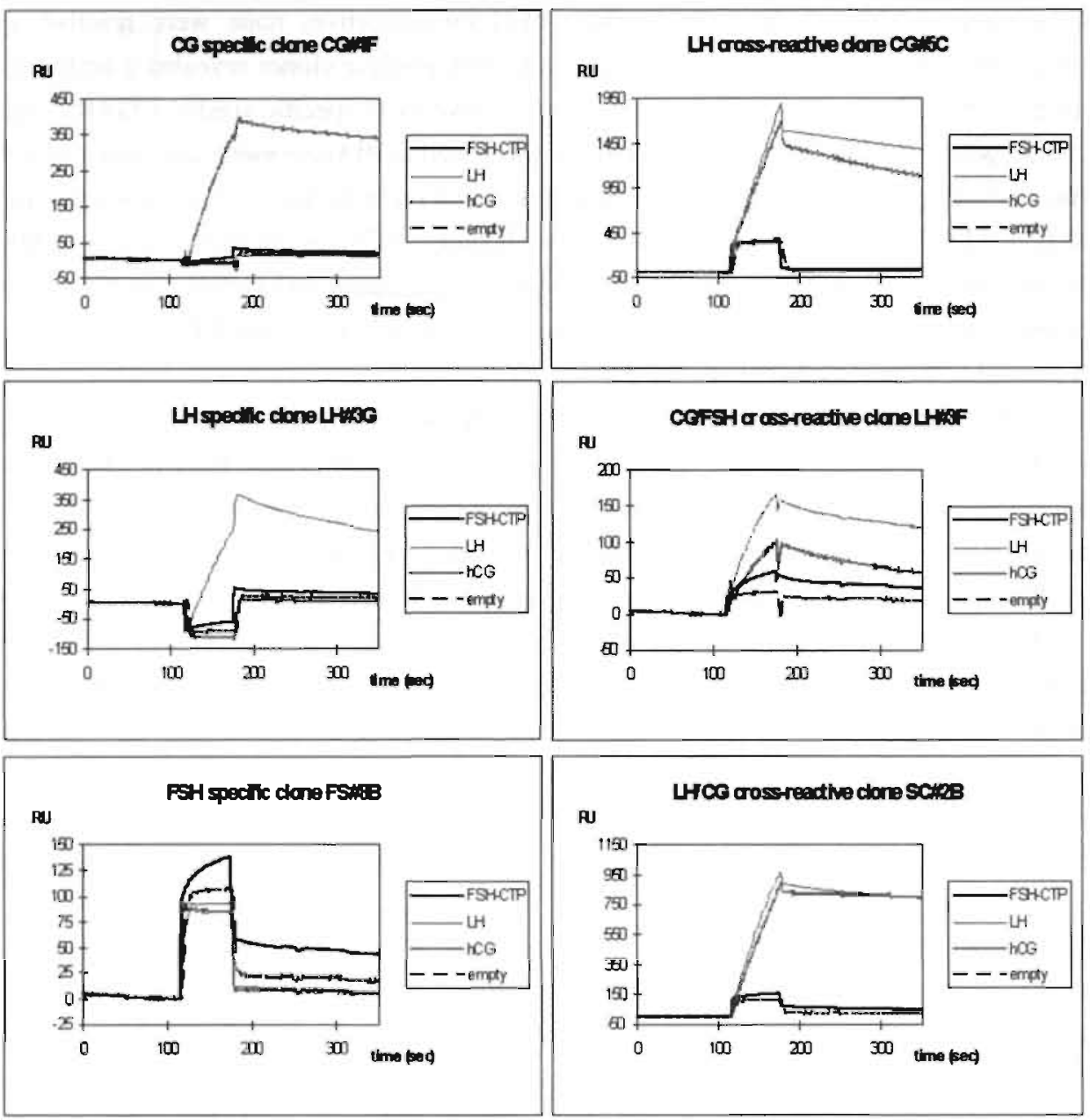

Figure 4. Analysis of specificity of selected anti-glycoprotein hormone Fab's using surface plasmon resonance. Periplasmic fractions from clones CG\#4F, CG\#5C. LH\#3G, LH\#3F, FS \#8B and $S C \# 2 B$ were injected at $\mathrm{t}=120 \mathrm{~s}$ on flow-cells with immobilised hFSH-CTP, hLH and hCG. and on an empty surface; at $t=180 \mathrm{~s}$ the injection phase was stopped and the cells were washed with HBS buffer. 
the selected Fab's. First, the nature of the Fab fragments in periplasmic fractions was determined in western blot. When incubated in non-reducing sample buffer, two products were detected with the $9 \mathrm{E} 10$ antibody, which recognises the myc-tag at the end of the $\mathrm{CHI}$ domain (Fig. 6A): the major product is the intact Fab-molecule, in which an intermolecular disutphide bridge
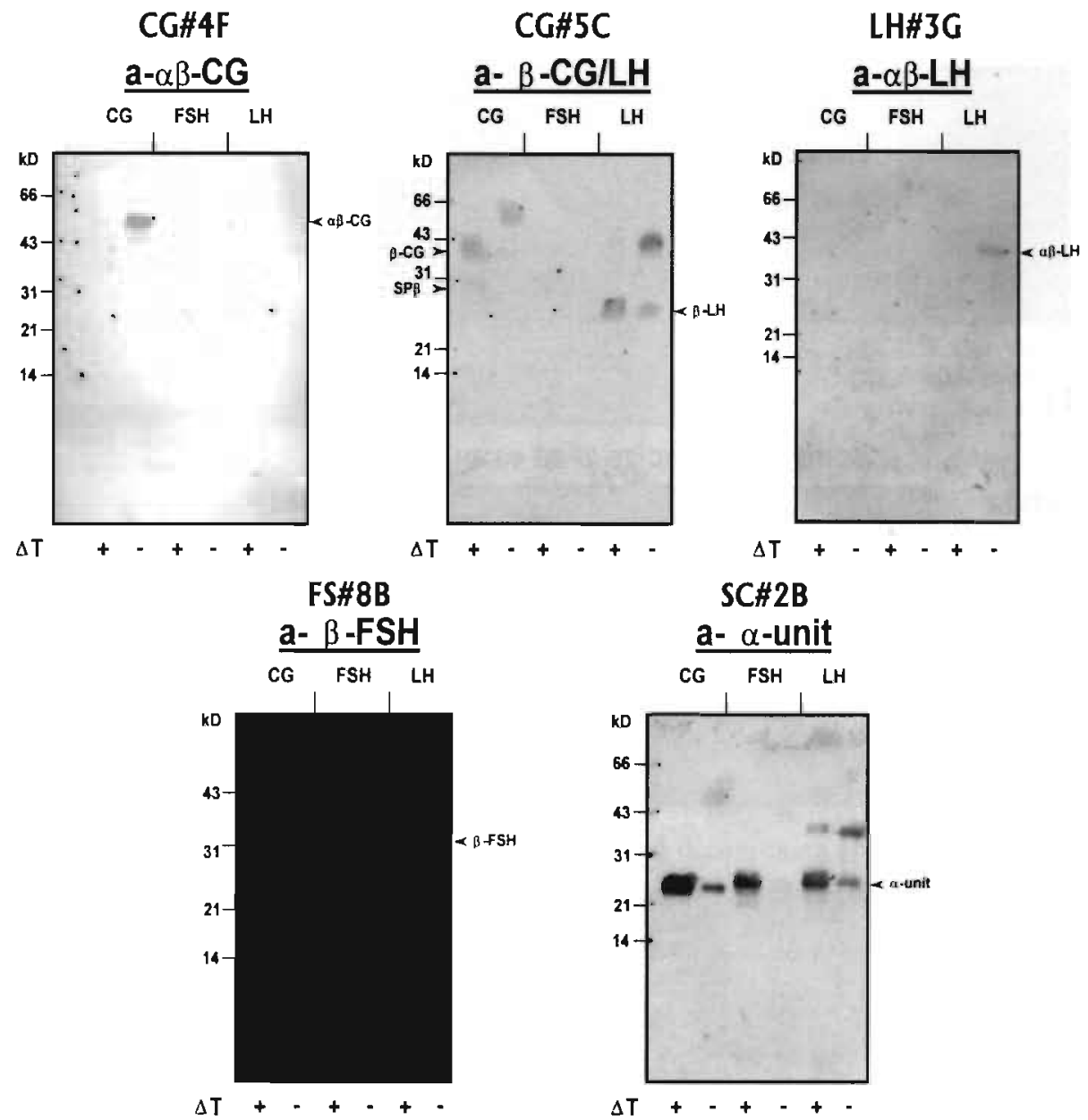

Figure 5. Specificities of the Fab's determined with western blot analysis. The glycoprotein hormones hCG. hFSH and hLH were diluted in non-reducing sample buffer, and boiled ( $\mathrm{T}+$ ) or not heat-treated ( $\mathrm{T}$-). loaded on a $15 \%$ SDS-PAGE, blotted onto nitrocellulose filter, and detected with the indicated Fab's. The molecular forms of the hormones recognised by the Fab's of clones CG\#4F, CG\#5C, LH\#3G, FS\#8B, and $\mathrm{SC} \# 2 \mathrm{~B}$, are indicated with arrows. 


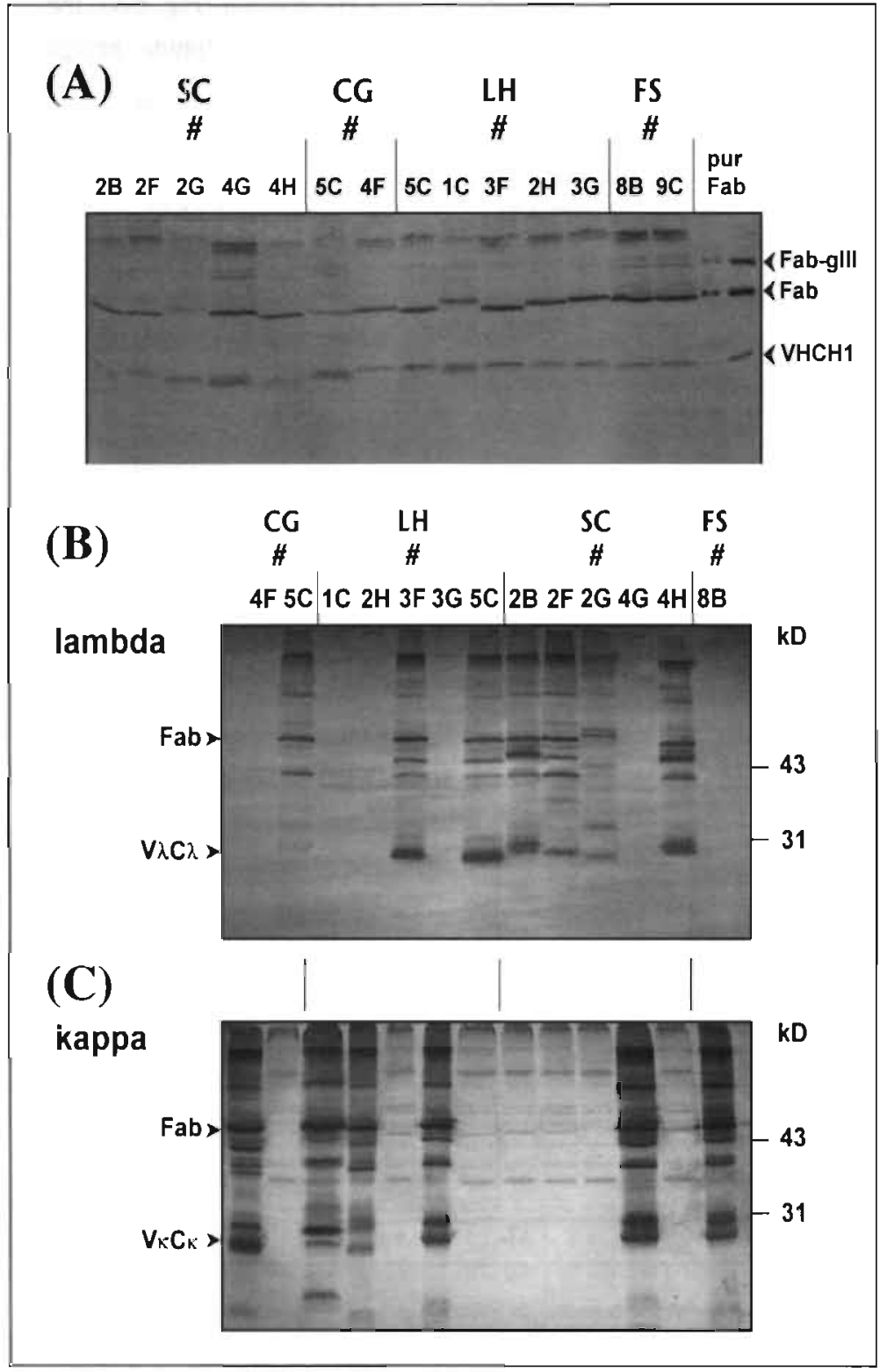

Figure 6. Examination of the integrity of produced Fab fragments on western blot. The periplasmic fractions from the indicated glycoprotein specific Fab clones were boiled in nonreducing sample buffer and loaded on 12.5\%, SDSPAGE. The blots were incubated with the anti-mvi mAB $9 E 10(A)$, anti-human lambda polyclonal antibodies $(B)$, or with anti-human kappa polyclonal antibodies (C). Affinity purified Fab was used as a control on the blot incubated with anti-myc $\mathrm{mAB}$ (indicated with pur Fab). 
covalently links heavy and light chain fragments; the low molecular product is most likely derived from non disulphide bridge linked heavy chains. Analysis with anti-light chain sera reveals a similar pattem and shows that the clones use a nearly equal percentage of kappa and

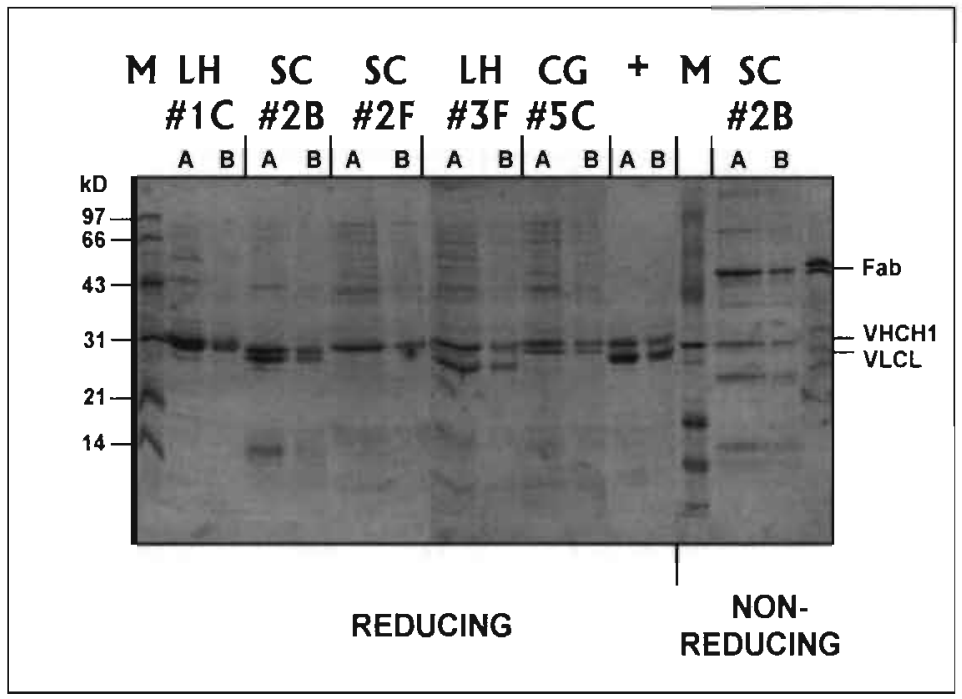

Figure 7. SDS-PAGE of affinity purified Fab's. hCG binding Fab's produced by clones LH\#1C, LH\#3F, CG\#5C, $\mathrm{SC} \# 2 \mathrm{~B}$ and $\mathrm{SCH} 2 \mathrm{~F}$ were purified by affinity chromatography on hCGsepharose, and analysed on a Coommasie stained $12.5 \%$ SDS-PAGE gel under reducing conditions, and for Fab $S C \# 2 B$ also under nonreducing conditions. As positive control, a Fab fragment made by proteolytic digestion of a human monoclonal antibedy, was included (marked with + ).

lambda chains (found in six and seven clones respectively of a total of 13 tested) (Fig. 6B,C). From the densities of the blots it would be unlikely that all of the light chain would be complexed as Fab. Instead, it appears that in many cases more light than heavy chain fragment is produced, which may be expected from the design of the bicistronic expression cassette. Upon purification of functional antigen-binding frag-ments using denaturation and refolding. followed by affinity chromatography, this excess of clones in Fig. 7). Upon reduction, equal amounts of heavy and light chain are seen, while under non-reducing conditions (shown for one clont only), the main product is represented by the disulphide linked Fab-molecule, with an equal amount of the (most likely) non-covalently linked VHCHI and VLCL products visible. Production yields of selected hormone specific Fab's varied between $160 \mu \mathrm{g}$ and $1.43 \mathrm{mg}$ Fab per litit' culturs: which was in the same range as was found for the unselected Fab's (data not shown).

\subsection{Use of diverse germline sequences}

A panel of 14 antigen-specific Fab's was fully scyuenced (Table IV; 3 anti-MIC 1 antibodies positive in BIAcore on 100-mer peptide; 11 anti-gonadotropin antibodies). The heavy chain genes are derived from the four largest VH families (VH1, VH3, VH4 and VHo): the VL. 
Table IV. V-gene segments and CDR3-sequences used by the selected Fab's

\begin{tabular}{|c|c|c|c|c|c|c|c|c|c|}
\hline clone & speclficity & $\begin{array}{l}\text { VH } \\
\text { family }\end{array}$ & $\begin{array}{c}\mathrm{VH} \\
\text { segment }\end{array}$ & CDR3 & $\begin{array}{l}\text { AA changes } \\
\text { from germline* }\end{array}$ & $\begin{array}{c}\text { VL } \\
\text { family }\end{array}$ & $\begin{array}{c}\text { VL } \\
\text { segmen } \\
t\end{array}$ & CDR3 & $\begin{array}{l}\text { AA changes } \\
\text { from germline }\end{array}$ \\
\hline MUC\#4 & MUC1 & $\mathrm{VH} 4$ & 4-39 & AAAGMVD & 7 & $V \times 2$ & A17 & MQATHWPPIT & 1 \\
\hline MUC\#9 & MUC1 & $\mathrm{VH} 4$ & $4-b / 4-39$ & PSIARAGQVY & 5 & $v_{\times 3}$ & L6 & QQYYSWPLT & 10 \\
\hline MUC\#32 & MUC1 & $\mathrm{VH} 3$ & $3-30$ & VGSGGWLYFDR & 2 & $V \times 3$ & L6 & QQRSHWPLT & 2 \\
\hline $\mathrm{CG} \# 4 \mathrm{~F}$ & $\beta-\mathrm{CG}$ & VH3 & $3-30.3$ & EGTATPGGTDY & 2 & $v_{\times 1}$ & L5 & QQSYSTPL & 7 \\
\hline CG\#5C & B-CG/LH & $\mathrm{VHA}$ & 4.04 & GAAASYYFDY & 0 & Vi. 6 & $6 a$ & QSSHSTAVV & 13 \\
\hline LH: $1 \mathrm{C}$ & $\alpha$ & VH1 & $1-08$ & GERSNFDY & 8 & $V_{k} 3$ & L6 & QHRRT & 8 \\
\hline $\mathrm{LH} \# 2 \mathrm{H}$ & $\alpha \beta$-LH & VH3 & 3-15 & DPGTIIYYYYGMDV & 0 & $v \times 2$ & A19 & MQALQAPLT & 4 \\
\hline LHEI3F & $\alpha$ & $\mathrm{VH} 3$ & $3-30.5$ & LYGDYVSAFDI & 2 & V. 7 & $7 b$ & LLYYGGARGV & 6 \\
\hline LH\#3G & $\alpha \beta-L H$ & $\mathrm{VH} 3$ & 3-15 & RIAAYYYYYGMDV & 0 & $v_{k 2}$ & A19 & MQALQTPRT & 0 \\
\hline FS $\# 8 B$ & $\beta$-FSH & VH6 & 6-01 & GEHGYTSS & 8 & $V_{k 2}$ & A19 & MQALQTPPT & 1 \\
\hline SCH2B & $\alpha$ & $\mathrm{VH} 1$ & 1-02 & GKVGASPDY & 3 & $\mathrm{v}_{2}$ & $2 \mathrm{a} 2$ & SSYTNSGTLV & 9 \\
\hline $\mathrm{SC} \# 2 \mathrm{~F}$ & $\alpha$ & $\mathrm{VH} 1$ & $1-02$ & ASGYFPNDAFDI & 6 & $\mathrm{~V} \lambda l_{1}$ & $1 \mathrm{c}$ & ESLDDSLDGVV & 20 \\
\hline $\mathrm{SCH} 2 \mathrm{G}$ & $\alpha$ & VH1 & $1-\theta$ & GEASYGAYNWFDP & 5 & $v_{2} 2$ & $2 e$ & CSYAGSNTWV & 9 \\
\hline $\mathrm{SCH} 4 \mathrm{G}$ & $\alpha$ & VH1 & 1.24 & GGYSGLA & 2 & $V \times 2$ & A19 & MQTLQPPWT & 2 \\
\hline
\end{tabular}

*Amino acid differences in V-gene segment, excluding the FR-l region encoded by the primers used for cloning, and CDR3.

genes belong to one of four $\mathrm{V} \kappa$-families or one of three $\mathrm{V} \lambda$-families. Chain promiscuity is seen for the $\alpha$-chain specific clone SCH4G, the $\alpha / \beta-\mathrm{LH}$ specific clones $\mathrm{LH} \# 2 \mathrm{H}$ and $\mathrm{LH} \# 3 \mathrm{G}$, and $\beta$ FSH specific clone FS\#8B, which all used a highly homologous VK2 light chain gene segment (A19, previously coded DPK15) combined with different heavy chain fragments. This promiscuity for A 19 was previously found in antibodies derived from a synthetic Fab repertoire (30). The 3 anti-MUCl antibodies use heavy and light chain genes derived from 2 different VH and $V \kappa$ families; clone MUC\#9 uses a VH with a cross-over of 2 segments. It is remarkable that both MUC\#4 and MUC\#9 VH genes use the same reading frame of the same D-segment (D6-13; with a stretch of 13 or $16 \mathrm{bp}$ from this segment, respectively (46)), encoding an alanine-glycine stretch (AAAG; Table IV). This may reflect a similar mode of binding to $\mathrm{MUCl}$, despite the use of a different light chain by these two clones.

\subsection{Measurement of affinities with purified anti-hCG Fab's}

The affinities and off-rates of affinity purified hCG reactive Fab's LH\#IC, SC\#2B, LH\#3F and $C G \# 5 C$ were determined. The off-rates for most Fab's were in the order of $10^{-2}$ and $10^{-3} \mathrm{~s}^{-1}$ (Table V). The off-rate values obtained using crude periplasmic fractions were in good agrement with the values found for the purified Fab's, validating the utility of the off-rate screen with unpurified Fab fragments. The affinities. $23 \mathrm{nM}$ and $38 \mathrm{nM}$ for the $\alpha$-subunit specific antibody $\mathrm{LH} \# \mathrm{IC}$ and the $\beta$-subunit $\mathrm{hCG} / \mathrm{hLH}$-cross reactive antibody $\mathrm{CG} \# 5 \mathrm{C}$ respectively, are 
comparable to the affinity of antibodies selected from a murine immune phage antibody library (H.d.H., B. Kazemier, et al.. unpublished); the top affinity, $2.7 \mathrm{nM}$ for the $\alpha$-chain specific Fab SC\#2B (Table V), approaches the values of the best anti-hCG monoclonal antibodies (H.d.H., B. Kazemier, et al.. unpublished).

Table V. Affinities of anti-hormone Fab's for $h C G$

\begin{tabular}{|c|c|c|c|c|}
\hline Clone & Preparation & $\begin{array}{l}k_{0 \pi} \\
\left(s^{-1}\right)\end{array}$ & $\left(\mathbf{M}^{-1} \cdot s^{-1}\right)$ & $\begin{array}{l}\mathbf{K}_{\mathbf{d}} \\
(\mathbf{M}) \\
\end{array}$ \\
\hline LH\#IC & $\begin{array}{c}\text { purified } \\
\text { periplasmic fraction }\end{array}$ & $\begin{array}{l}(1.04 \pm 0.04) \times 10^{-2} \\
(1.12 \pm 0.08) \times 10^{-2}\end{array}$ & $\begin{array}{c}(4.50 \pm 0.37) \times 10^{5} \\
\text { nd }\end{array}$ & $(2.30+0.23) \times 10^{-8}$ \\
\hline $\mathrm{SC} \# 2 \mathrm{~B}$ & $\begin{array}{c}\text { purified } \\
\text { periplasmic fraction }\end{array}$ & $\begin{array}{l}(2.89 \pm 0.41) \times 10^{-3} \\
(2.90 \pm 0.57) \times 10^{3}\end{array}$ & $\begin{array}{c}(1.06 \pm 0.08) \times 10^{6} \\
\text { nd }\end{array}$ & $(2.71 \pm 0.25) \times 10^{11}$ \\
\hline CG\#5C & $\begin{array}{c}\text { purified } \\
\text { periplasmic fraction }\end{array}$ & $\begin{array}{l}(1.46 \pm 0.27) \times 10^{-2} \\
(1.21 \pm 0.16) \times 10^{-2}\end{array}$ & $\begin{array}{c}(3.76 \pm 0.71) \times 10^{5} \\
\text { nd }\end{array}$ & $\begin{array}{c}(3.88 \pm(1) .03) \times 10 \\
\text { nd }\end{array}$ \\
\hline $\mathrm{LH} \# 3 \mathrm{~F}$ & periplasmic fraction & $(5.84 \pm 0.08) \times 10^{-3}$ & nd & nd \\
\hline $\mathrm{CG} \# 4 \mathrm{~F}$ & periplasmic fraction & $(2.22 \pm 0.28) \times 10^{-3}$ & nd & nd \\
\hline $\mathrm{SC \# 2F}$ & periplasmic fraction & $2.25 \times 10^{3}$ & nd & nd \\
\hline
\end{tabular}

nd, not determined.

\section{Discussion}

This report describes the construction of a phage display library from the in vivo rearranged $\mathrm{V}$-gene repertoire of human donors and its evaluation by selection with a panel of hapten and protein antigens. The source of antibody producing B-cells was two-fold: peripheral blood lymphocytes, which are mainly IgM-positive cells, and B cells from a human spleen. We employed an efficient two-step cloning procedure with DNA-fragments digested from plasmid DNA instead of PCR fragments, to obtain the largest naïve human Fab repertoire reported to date, with a theoretical diversity of 37 billion different clones.

The choice of the Fab format was based on the possibility to develop rapid affinity/kinetic screens. Most large libraries made to date use the single chain format for display on phage $(7,20)$. One report described the use of a human naive Fab library on phage (not permitting immediate screening of selected soluble Fab fragments) (30). scFv's have the tendency to form dimers and higher order multimers in a clone-dependent and relatively unpredictable way (31,32.47). As a consequence, the affinity assay used (such as BIAcore analysis) often necessitates purification of the selected antibody fragments. For example, ranking for off-rates using BIAcore is not easily possible with unpurified scFv fragments; the monomeric fraction of 
selected scFv clones first needs to be purified by affinity chromatography and gel-filtration $(20,48)$. Our data suggest that the off-rate screening of individual Fab clones using non-purified bacterial preparations yield data similar to the off-rates determined with the purified Fab fragments. Therefore, provided sufficient Fab fragment is produced, the true monomeric appearance of Fab's allows a rapid initial screen for off-rate. In combination with a concentration determination assay (which could also be carried out on BIAcore (29)), this should allow the rapid affinity determination of large series of antigen-specific Fab's. The Fab format is therefore more amenable then scFv fragments to high throughput affinity screening, and should be the preferred format when rapid affinity measurement is crucial (e.g. during affinity maturation studies).

As was postulated and observed by Griffiths and colleagues (30), the size of the antibody library dictates the probability of the selection of high affinity antibodies to the antigen. Comparison of the first naive scFv repertoire containing $2.9 \times 10^{7}$ clones (18), with a recently constructed ScFV repertoire of approximately $10^{10}$ clones $(7,20)$, confirms this postulation: increasing the library size 500-fold resulted in approximately 100 -fold higher affinities. This increase is caused by lowering the off-rates from $10^{-1}-10^{-2} \mathrm{~s}^{-1}$ for fragments selected from the smaller sized library to $10^{-3}-10^{-4} \mathrm{~s}^{-1}$ for those from the larger library. This is in the same order of magnitude as we observe for the off-rates of our selected antibody fragments. Since Fab fragments lack the tendency to dimerise, Fab libraries could possibly display a lower fraction of avid phage than equivalent scFv libraries library. This does not appear to have reduced the number nor average affinity of selected antibodies. An indication that antibodies from this library behave similarly or better with regards to affinity comes from a comparison of selections of two different libraries on the same two antigens under identical conditions. Antibodies to MUCl selected from a large naïve scFv library (24) have faster off-rates then the equivalent Fab's isolated from the library described in this study. Further, they show a very distinct V-gene usage and have a different fine specificity (P.H., H.R.H. et al., unpublished). Similarly, when comparing the off-rates of phage antibodies against the pancarcinoma marker Epithelial Glycoprotein-2. One of the Fab's selected from the present library appears to have a 10-fold slower off-rale than the best scFv (7) (R.C.R., H.R.H. et al., in preparation).

The aflinities of the selected antibody fragments is, however, very much dependent on the antigen used for selection. Sheets and colleagues reported an affinity varying between 26 and 71 $\mathrm{nM}$ for the selected scFv fragments specific for the anti-Clostridia botulinum neurotoxin type A fragments, whereas for antibodies to the extracellular domain of human ErbB-2, $K_{i}$ 's between 0.22 and $4.03 \mathrm{nM}$ were found (20). The affinities of the gonadotropin specific Fab's selected from our library varied between 2.7 and $38 \mathrm{nM}$, which is comparable to the protein binding scFv's from the naïve library made by Vaughan et al. (7) and Sheets et al. (20), and approaches the values of the best antibodies in their kind (H.d.H, Kazemier et al., unpublished). 
The size of the library is not only important for affinity, but it also determines the success rate of selection of antibodies against a large set of different antigens. In this respect the library performs very well: over 24 antibodies to the hapten $\mathrm{phOx}$, and on average 13 antibodies against the other antigens were selected. Furthermore, the specificities of the antibodies obtained by selections on the gonadotropins are unique: due to the high degree of homology between hLH and hCG it has been very difficult to isolate hCG specific monoclonal antibodies with the hybridoma technology, whereas there are very few hLH specific antibodies $(27,39)$. Using a straight forward selection procedure, taking no precaution to avoid the selection of cross-reactive Fab's, we have readily isolated fragments with all pussible specificities: Fab's specific for any of the three hormones hCG, hLH and hFSH, and cross-reactive Fab's recognizing the common $\alpha$ chain or epitopes on the $\beta$-chain shared by hCG and hLH. These selections demonstrated that antibodies directed against different epitopes within single antigen molecules can be retrieved from the library.

In the limited set of 14 clones that were sequenced, we identify antibodies with variable region genes from all large $\mathrm{V}$-gene families, including $\mathrm{VH} 1 / 3 / 4, V \kappa 1 / 3$, and $V \lambda 1 / 2$, but also less frequently used segments of family VH6, V $22 / 7$ and $V \lambda 7$ were retrieved. Most likely the use of an extended set of variable region gene primers. designed on the most recent sequence information of the germline V-regions, and/or the separate PCRs, combined with partially separate cloning, ensured access to a highly diverse sample of the human $V$-gene repertoire.

The average amino acid mutation frequency of the selected human $\mathrm{V}$-genes was calculated to be the $3.95 \% ;$ for the VH segments (50 amino acid mutations in 1267 ) and $7.30 \%$ for the VL (92 out of 1260). This mutation frequency is reversed to what was reported for $\operatorname{IgG}+\mathrm{B}$-cells (49) ( $10 \%$ for $\mathrm{VH}$ and $6 \%$ for VL). The higher mutation rate of the VL-genes in the combinatorial repertoire may serve to compensate the rather restricted natural light chain diversity. As the overall natural light chain diversity is only a fraction of the heavy chain diversity, the use of spleen versus PBL-derived lights chains, may help to provide additional light-chain based combining site diversity.

With respect to the VH segments of the selected antibodies, the mutation frequency is remarkable lower (mean 3.57( \pm 2.90$)$ mutated residues per VH) than what was found in the selected scFv fragments by Vaughan and colleagues (7) (mean 7.53( \pm 4.25 ) mutated residues). The light chain segments seem to have similar mutation frequencies (Vaughan et al: $8.23 \pm 5.20$ residues, versus $6.57 \pm 5.56$ residues in the here reported Fab's). Probably the IgM-specific primer, used by us for PCR, resulted in the isolation of more "naïve" VH segments.

This new phage library will be a valuable source of antibodies to essentially any target. To date we have been able to select specific antibodies to over 20 antigens tested. The antibodies may be used as research reagents or as starting point for the development of therapeutic antibodies. As the list of sequenced genomes and disease-related gene products is expanding rapidly, there will be a growing need for an in vitro and eventually automated method for 
antibody isolation. As antibodies have been and will be ideal probes for investigating the nature, localisation and purification of novel gene products, this library is envisaged to play an important role in target validation and target discovery in the area of functional genomics.

\section{References}

1. Winter, G., Griffiths, A. D., Hawkins, R. E., and Hoogenboom, H. R. (1994) Ann. Rev. Immunol. 12, 433-455

2. Clackson. T., and Wells, J. A. (1994) Trends Biotechnol. 12, 173-184

3. Holliger, P. H., and Hoogenboom, H. R. (1998) Nat. Biotechnol. in press

4. Marks, J. D., Hoogenboom, H. R., Bonnert. T. P., McCafferty, J., Griffiths, A. D., and Winter, G. (1991) J. Mol. Biol. 222, 581-597

5. Hoogenboom, H. R., and Winter, G. (1992) J. Mol. Biol. 227, 381-388

6. Griffiths, A. D., Malmqvist, M., Marks, J. D., Bye, J. M., Embleton, M. J., McCafferty, J., Baier, M., Holliger, K. P., Gorick, B. D., Hughes, J. N., Hoogenboom, H. R., and Winter, G. (1993) EMBO J. 12, 725-734

7. Vaughan, T. P., Williams A.W., Pritchard, K., Osbourn, J. K., Pope, A. R., J.C., E., McCafferty, J., Hodits, R. A., Wilton, J., and Johnson, K. S. (1996) Nat. Biotechnol. 14, 309-314

8. Better, M., Chang, C. P., Robinson, R. R., and Horwitz, A. H. (1988) Science 240, $1041-1043$

9. Skerra, A., and Pluckthun, A. (1988) Science 240, 1038-1041

10. Larrick, J. W., Danielsson, L., Brenner, C. A., Abrahamson, M., Fry, K. E., and Borrebaeck, C. A. (1989) Biochem. Biophys. Res. Commun. 160, 1250-1256

11. Ward, E. S., Gussow, D., Griffiths, A. D., Jones, P. T., and Winter, G. (1989) Nature 341, 544-546

12. Marks, J. D., Tristem, M., Karpas, A., and Winter, G. (1991) Eur. J. Immunol. 21, 985 991

13. Clackson, T., Hoogenboom, H. R., Griffiths, A. D., and Winter, G. (1991) Nature 352, 624-628

14. Persson, M. A., Caothien, R. H., and Burton, D. R. (1991) Proc. Natl. Acad. Sci. U. S. A. 88, 2432-2436

15. Orum, H., Andersen, P. S., Oster, A., Johansen, L. K., Riise, E., Bjornvad, M., Svendsen, I., and Engberg, J. (1993) Nucleic Acids Res. 21, 4491-4498

16. Gram, H., Marconi, L., Barbas, C. F., Collet, T. A., Lemer, R. A., and Kang, A. S. (1992) Proc. Natl. Acad. Sci. U. S. A. 89, 3576-3580

17. Klein, U., Kuppers, R., and Rajewsky, K. (1997) Blood 89, 1288-1298

18. Marks, J. D., Hoogenboom, H. R., Bonnert, T. P., McCafferty, J., Griffiths, A. D., and 
Winter, G. (1991) J. Mol. Biol. 222, 581-597

19. Nissim, A., Hoogenboom, H. R., Tomlinson, I. M., Flynn, G., Midgley, C., Lane, D., and Winter, G. (1994) EMBO J. 13, 692-698

20. Sheets, M. D., Amersdorfer, P., Finnern, R., Sargent, P., Lindquist, E., Schier, R., Hemingsen, G., Wong, C., Gerhart, J. C., and Marks, J. D. (1998) Proc. Natl. Acad. Sci. U. S. A. 95, 6157-6162

21. Chirgwin, J. M., Przybyla, A. E., MacDonald, R. J., and Rutter, W. J. (1979) Biochemistry 18, 5294-5299

22. Persic, L., Roberts, A., Wilton, J., Cattaneo, A., Bradbury, A., and Hoogenboom. H. R. (1997) Gene 187, 9-18

23. Hawkins, R. E., Russell, S. J., and Winter, G. (1992b) J. Mol. Biol. 226, 889-896

24. Henderikx, P., Kandilogiannaki, M., Petrarca, C., von Mensdorff-Pouilly. S., Hilgers, J. H., Krambovitis, E., Arends, J. W., and Hoogenboom, H. R. (1998) Cancer Res. 58, 4324-4332

25. Matzuk, M. M., Spangler, M. M., Camel, M., Suganuma, N.. and Boime, I. (I989) J. Cell. Biol. 109, 1429-1438

26. Muyan, M., Furuhashi, M., Sugahara, T., and Boime, I. (1996) Mol. Endocrinol. 10, 1678-1687

27. Moyle, W. R., Matzuk, M. M., Campbell, R. K., Cogliani, E., Dean-Emig, D. M., Krichevsky, A., Barnett, R. W., and Boime, I. (1990) J. Biol. Chem. 265, 8511-8518

28. de Haard, J. J. W., Kazemier, B., van der Bent, A., Oudshoorn, P., Boender, P., van Gemen, B., Arends, J.-W., and Hoogenboom, H. R. M. (1998) Protein Eng. 11, 12671276

29. Kazemier, B., de Haard, H., Boender, P., van Gemen, B., and Hoogenboom, H. R. (1996) J. Immunol. Methods 194, 201-209

30. Griffiths, A. D., Williams, S. C., Hartley, O., Tomlinson, I. M., Waterhouse, P., Crosby, W. L., Kontermann, R., Jones, P. T., Low, N. M., Allison, T. J., Prospero, T. D., Hoogenboom, H. R., Nissim, A., Cox, J. P. L., Harrison, J. L., Zaccolo, M., Gherardi, E., and Winter, G. (1994) EMBO J. 13, 3245-3260

31. Weidner, K. M., Denzin, L. K., and Voss, E. W., Jr. (1992) J. Biol. Chem. 267, 10281 10288

32. Holliger, P., Prospero, T., and Winter, G. (1993) Proc. Natl. Acad. Sci. U. S. A. 90, 6444-6448

33. Kortt, A. A., Lah, M., Oddie, G. W., Gruen, C. L., Burns, J. E., Pearce, L. A., Atwell, J. L., McCoy, A. J., Howlett, G. J., Metzger, D. W., Webster, R. G., and Hudson, P. J. (1997) Protein Eng. 10, 423-433

34. Hoogenboom, H. R., Griffiths, A. D., Johnson, K. S., Chiswell, D. J., Hudson, P., and Winter, G. (1991) Nucleic Acids Res. 19, 4133-4137 
35. Hochuli, E., Bannwarth, W., Döbeli, H., Gentz, R., and Stüber, D. (1988) BioTechnology 6, 1321-1325

36. Munro, S., and Pelham, H. R. (1986) Cell 46, 291-300

37. Roit, I. M., Brostoff, J., and Male, D. K. (1985) Immunology, Gower Medical Publishing, Ltd., London

38. Griffiths, G. M., Berek, C., Kaartinen, M., and Milstein, C. (1984) Nature 312, 271 275

39. Cole, L. A. (1997) Clin. Chem. 43, 2233-2243

40. Masure, H. R., Jaffee, W. L., Sickel, M. A., Birken, S., Canfield, R. E., and Vaitukaitis, J. L. (1981) J. Clin. Endocrinol. Metab. 53, 1014-1020

41. Papapetrou. P. D., Sakarelou, N. P., Braouzi, H., and Fessas, P. (1980) Cancer 45, 2583-2592

42. Fares, F. A., Suganuma, N., Nishimori, K., LaPolt, P. S., Hsueh, A. J., and Boime, I. (1992) Proc. Natl. Acad. Sci. U. S. A. 89, 4304-4308

43. Schier, R., and Marks, J. D. ( 1996) Hum. Antibodies Hybridomas 7, 97-105

44. Cai, X., and Garen, A. (1995) Proc. Natl. Acad. Sci. U. S. A. 92, 6537-6541

45. Gargano, N., Biocca, S., Bradbury, A., and Cattaneo, A. (1996) J. Virol. 70, 7706-7712

46. Corbett, S. J., Tomlinson, I. M., Sonnhammer, E. L. L., Buck, D., and Winter, G. (1997) J. Mol. Biol. 270, 587-597

47. Marks, J. D., Ouwehand, W. H., Bye, J. M., Finnern, R., Gorick, B. D., Voak, D., Thorpe, S. J., Hughes, J. N., and Winter, G. (1993) Biotechnology N Y 11, 1145-1149

48. Schier, R., Bye, J., Apell, G., McCall, A., Adams, G. P., Malmqvist, M., Weiner, L. M., and Marks. J. D. (1996) J. Mol. Biol. 255, 28-43

49. de Wildt, R. M. T., Hoet, R. M. A., van Venrooij, W. J., Tomlinson, I. M., and Winter, G. (1998) J. Mol. Biol. in press

\section{ACKNOWLEDGEMENTS}

We thank colleagues at the Department of Pathology, and in particular Dr. A. P. de Bruïne and Dr. R. Hoet and Mrs. C. Petrarca for discussions, and collaborators (Dr. E. Krambovitis) for materials. P.H. has been supported by a grant from the European Community, Biotechnology Programme 5.1 (BIO4CT950252). 
SUMMARY; SAMENVATTING 


\section{Exploring Antibody Engineering Technology for diagnostic applications; summary}

As was stated in the Introduction, the principal aim of the thesis was to determine the value of phage display and engineering technology in the generation and modification of antibodies for diagnostic purposes. The evaluation was performed in the field of endocrinology, for diagnosis of pregnancy, and in the area of virology for the detection of the AIDS-causing Human Immunodeficiency Virus type 1 (HIV-1). The immunoassays dedicated to the detection of hormones and viruses have to be specific and sensitive, characteristics which are determined by the used antibodies. Therefore, the library derived antibody fragments were analysed on these features and compared with monoclonal antibodies made via traditional methods.

\section{I Library derived anti-hCG scFv-s}

The display of antibodies on the filamentous bacteriophage M13 was set up in our Jaboratory with hybridoma produced monoclonal antibodies reactive against the hormone hormone human Chorionic Gonadotropin (hCG). The idea is obvious: the combination of one type of heavy chain variable region with its partner light chain variable region within a single chain antibody, should yield antigen reactive phage, when the recombinant molecule was expressed on its surface. However, we encountered problems when using several anti-hCG and anti-HIV-1 antibodies, in that we never found antigen binding phage and derived antibody fragments.

By application of mutant analysis, as described in Chapter 1, it was concluded that a single mutation within the Frame work region 1 of the heavy chain variable region $\left(V_{H}\right)$, introduced by the oligonucleotide primer used for amplification of the $\mathrm{V}$ regions, was responsible for this failure. Three studied anti-hCG antibodies with a subgroup IIA $\mathrm{V}_{\mathrm{H}}$, completely lost their capacity to bind antigen, when at position 6 the wild type residue glutamine (Q) was substituted by glutamic acid $(E)$, a residue found frequently in murine antibodies also. The inactive mutant $s c{ }^{i} v-s$ tumed out to be more sensitive for trypsin digestion than the wild type derivatives, which suggests incorrect folding of the mutant fragments.

Grafting of three residues located within the first two $\beta$-strands of the $\mathrm{V}_{\mathrm{H}}$-domain, that distinctively occur in antibodies with a of subgroup IA $V_{H}$ and thereby having glutamic acid as number 6 residue, onto the "defect" subgroup IIA sequence partially restored activity of the Q6E containing scFv. Possibly the involved residue may be part of a folding nucleus, and considering the evolutionary conservation of either glutamine or glutamic acid in the two murine antibody families, this folding nucleus might even be family specific.

A similar effect on antigen binding, caused by a mutation in the Frame work region 1 of $V_{\kappa}$, was discussed in Chapter 2. When the wild type residue methionine at position 4 was substituted by serine in two anti-hCG antibodies having a subgroup II kappa light chain, antigen-binding 
could not be detected in ELISA. However, the same replacement within an anti-hCG antibody with a subgroup IV kappa light chain, and thereby having leucine as wild type residue, did not affect the binding characteristics. In contrast with the above described $\mathrm{V}_{\mathrm{H}}$-mutation, a low affinity binding could be detected by surface plasmon resonance on a high density surface of antigen. An intermediate trypsin resistance might be an indication for a more local distortion of the conformation rather than a completely misfolded antibody domain, which was observed with the previously described $\mathrm{V}_{\mathrm{H}}$-mutation. The local effect might influence the canonical structure of the nearby located L1, and resulting from this, the binding affinity.

During both studies it was important to quantify the amount of actively binding scFv in a crude periplasmic fraction. In Chapter 3, a method was developed where the concentration of antigen binding scFv was measured with surface plasmon resonance on BIAcore under mass transfer limitation conditions. When $s c F v$ was injected on a high density antigen surface, a linear relationship was obtained between the binding rate and the concentration of the antibody fragment. After construction of a single calibration curve with purified scFv. the amount of antigen binding antibody fragment in a sample could be determined rapidly by its binding rate, acquired from injection on the same surface.

For diagnosis of pregnancy, elevated levels of the glycoprotein hormone human Chorionic Gonadotropin (hCG) in urine are measured in immunoassays. Complicating factors are the presence of the closely related human Luteinizing Hormone (hLH) and of free subunits or fragments derived from the $\beta$-chain of hCG. Therefore a combination of monoclonal antibodies has to be used, by which only intact hCG will be detected. Especially cross-reactivity against $\mathrm{hLH}$ forms a severe problem, since both hCG and hLH use an identical $\alpha$-chain, and their $\beta$ chains are $85 \%$ homologous in respect to the first 114 amino acid residues. In this context we applied phage display technology for the selection of hCG specific scFv-s. Chapter 4 describes the use of libraries, generated from the spleens of two immunised mice, for the selection of different anti-hCG scFv-s. Analysis with surface plasmon resonance and Western blot using the recombinant antibodies as detection probes, we identified a group of anti- $\beta$-core fragment, $h C G$ specific antibodies, as well as an antibody only recognising the bioactive molecular form of $\mathrm{hCG}$, being the intact heterodimer. In spite of its cross-reactivity against hLH, its use in an assay in combination with the hCG-specific antibody fragment would detect the bioactive molecular form of the hormone specifically.

ScFv-s with other specificities were also selected, such as anti- $\alpha$-subunit specific antibodies, and a group of anti- $\beta$-core, hLH cross reactive fragments. Comparison of the library derived scFv-s with those of monoclonal antibodies, which are included in a commercially available pregnancy assay, showed that both groups have similar affinities. Therefore, the same sensitivity can be expected for an assay, which will contain the recombinant antibody fragments. 


\section{I.2 Anti-HIV-I sc F $\mathrm{v}^{\prime}-\mathrm{s}$}

The presence of Human Immunodeficiency Virus type 1 (HIV-1) in human sera is diagnosed most frequently on the major capsid protein p24. The structural protein is abundantly present in the viral genome containing capsid. Screening with p24-antigen assays enables an early identification of sera from infected individuals: with currently used immunoassays, the presence of p24 antigen maly be assessed one to two weeks earlier than an antibody response against the virus. Testing on 24 is important also during treatment of patients with anti-viral drugs. The spontaneous generation of drug resistant variants leads to a reactivation of virus. In order to detect the virus in an early stage of the reactivation phase, but also during the primary infection, the applied monoclonal antibodies must have high affinities, since this feature is directly correlated with the sensitivity of the assay. Because of the heterogeneity of the different HIVstrains, the antibodies also have to recognise conserved epitopes within $\mathrm{p} 24$.

The selection of a number of anti-p24 scFv-s from an immune repertoire is presented In Chapter 5. After immunisation of a mouse with HIV-1 viral lysate. RNA was extracted from its spleen and used for the construction of the repertoire. One of the selected antibodies recognised an immunodominant epitope of $\mathrm{p} 24$, as could be concluded from pepscan analysis. A previously described hybridoma produced monoclonal antibody, with the same specificity, used the identical combination of $\mathrm{V}_{\mathrm{H}^{-}}$and $\mathrm{V}_{\mathrm{K}}$-regions. Therefore this $\mathrm{scFv}$ is one of the rare examples where the original pairing in a library derived antibody fragment was retrieved. The affinities of the selected fragments were comparable to those of monoclonal antibodies, that are currently applied in a commercial p24 antigen assay.

Instead of immunising mice, a human repertuire was made for the selection of antiHIV-1 envelope antihodies. Chapter 6 discusses the generation of the scFv-library from just a small number of peripheral blood lymphocytes, donated by a seropositive individual. An important argument for selection of human antibodies is the difference in antigen presentation during immunisation of a mouse, when compared with virus replication in man. Also, false positive ELISA signals, observed with sporadically occurring sera containing human anti-mouse antiburles' (HAMA), may be avoided by the application of human antibodies in ELISA for capturing and/or detection.

Biopanning with the envelope precursor gp 160 resulted in the selection of two families of scFv-s. One group recognised a conformational epitope within the mature envelope protein gp 120, the other family was specific for the transmembrane glycoprotein gp 41 ; both structural proteins are cleaved by proteolysis from gpl60. Due to a sequence homology with previously described library derived Fab-s, it was concluded that our scFv-s react with the cluster III epitope of gp $4 \mathrm{l}$.

Competition ELISA showed that especially the epitope of the $\alpha$-gp4l antibodies was recognised by a broad panel of patient sera from North American and African seropositives. Due 
to the conserved nature of its epitope, the anti-gp4l scFv seems to be the most promising candidate for application in in vitro diagnostics.

\subsection{Construction of and selections from a nä̈ve human repertoire}

The rather time-consuming immunisation and the repeated construction of immune repertoires, needed for the selection of the anti-hCG- and anti-HIV-1 -antibodies discussed in the previous Chapters, can be completely superseded by the application of a naive repertoire. It has been reported, that depending on the library size, high affinity antibodies against any antigen can be retrieved from such repertoires.

Chapter 7 presents the construction of a huge naive Fab repertoire, containing 37 billion phage clones. The quality was analysed by selection with two protein antigens, i.e., tetanus toxoid and the tumor associated antigen mucine-1, and the hapten phOx, and by the following affinity analysis of the selected Fab-s. As measure for the affinity, the off-rates were determined with surface plasmon resonance by injection of crude periplasmic fractions. which followed monophasic kinetics due to the purely monomeric appearance of the Fab-s.

The real challenge was formed by selection of the naive repertoire with the glycoproteins hormones, a theme, which is running through this thesis like a continuous thread (Chapter 1 to 4). Upon selection with human Chorionic Gonadotropin (hCG) a group of Fab's was found, recognising the holo-form of this hormone specifically, as well as $\beta$-unit specific Fab's, which were cross reactive against human Luteinizing Hormone $(\mathrm{hLH})$. With hLH as target molecule for selection, we retrieved Fab's, recognising the heterodimer form of the target hormone only, and a group of antibodies reactive against the $\alpha$-subunit, and therefore cross-reactive against the other members of the family of glycoprotein hormones. Application of human Follicle Stimulating Hormone (hFSH) resulted in the isolation of $\beta$-subunit, hormone specific antibodies and $\alpha$-unit hLH/hCG cross reactive Fab's. From all hCG-reactive antibody fragments, purified with antigen columns, we determined the kinetics of binding to antigen with surface plasmon resonance. The affinity varied between 2.7 and $38 \mathrm{nM}$, comparable with antibodies obtained from an immune repertoire or produced by hybridoma cell lines. 


\section{Samenvatting: evaluatie van Antibody Engineering Technologie voor toepassing in diagnostika}

Zoals in de Inleiding reeds werd vermeld, was het belangrijkste doel van dit promotie onderzoek het belang van faag display en genetische manipulatie vast te stellen voor het maken en de modifikatie van antilichamen, die diagnostisch toegepast worden. De evualatie vond plaats op het gebied van de endocrinologie, voor het vaststellen van zwangerschap, en op het gebied van de virologie, voor de detektie van het Human Immunodefiency Virus type I (HIV-1), welke de ziekte AIDS veroorzaakt. De immunologische testen, die gebruikt worden voor de detektie van hormonen en virussen, moeten specifiek en gevoelig zijn, karakteristieken die bepaald worden door de in de test gebruikte antilichamen. Daarom werden de antilichaam fragmenten, die geïsoleerd werden uit de faag banken, geanalyseerd op deze beide eigenschappen en vergeleken met monoklonale antilichamen, die met traditionele methoden gemaakt zijn.

\subsection{Anti-hCG scFv-s geïsoleerd uit een faag bank}

De display van antilichamen op de filamenteuze bacteriofaag M13 is binnen onze groep opgezet met door hybridoma's geproduceerde antilichamen, die tegen het hormoon human Chorionic Gonadotropin (hCG) gericht zijn. Het achterliggende idee ligt voor de hand: de kombinatie van een type zware keten variabele regio met de bijbehorende lichte keten variable regio in één enkelketenig antilichaam fragment, ofwel "single chain Fv" (scFv), moet een antigeen specifieke faag opleveren, wanneer het rekombinante molekuul op diens oppervlak tot expressie wordt gebracht. Wij hadden echter veel problemen bij het omzetten van een aantal verschillende anti-hCG en anti-HIV-1 monoklonale antilichamen naar in $E$. coli geproduceerde antilichaam fragmenten: we vonden nooit antigeen bindende fagen en hiervan afgeleide antilichaam fragmenten.

Door de analyse van mutanten, welke beschreven is in Hoofdstuk 1, kon worden aangetoond, dat één enkele mutatie binnen Frame work regio 1 van de zware keten variabele regio $\left(V_{H}\right)$, geïntroduceerd door een oligonucleotide primer, die gebruikt was voor de amplifikatie van de V-regio's, verantwoordelijk was voor deze mislukking. Drie bestudeerde anti-hCG antilichamen met een groep IIA $V_{H}$ verloren in het geheel het vermogen om antigeen te binden, wanneer op positie 6 het wild type residu glutamine $(\mathrm{Q})$ vervangen was door glutamaat (E), een residu dat ook veelvuldig voorkomt in muis antilichamen. De inaktieve muatnt scFv-s bleken gevoeliger te zijn voor digestie met trypsine dan hun wild type afgeleiden, wat een verkeerde opvouwing van de mutant fragmenten suggereert.

"Transplantatie" van drie residuën uit de eerste twee $\beta$-strands van het $V_{H^{-}}$-domein, die uitsluitend voorkomen in antilichamen met een groep IA $V_{H}$, en die derhalve glutamaat als positie 6 residu hebben, naar de "defekte" groep IlA sequentie herstelde de aktiviteit van de 
Q6E-mutant scFv gedeeltelijk. Waarschijnlijk maakt het bestudeerde positie 6 residu deel uit van een vouwingskern ("folding nucleus"), die, gezien de evolutionaire onveranderlijkheid van ofwel glutamaat of glutamine in de twee muis antilichaam families, wellicht familie specifiek is.

In Hoofdstuk 2 is een gelijkend effekt op antigeen binding beschreven, die werd veroorzaakt door een mutatie in de Frame work regio 1 van $V_{k}$. Wanneer het wild type residu methionine op positie 4 vervangen was door serine in twee anti-hCG antilichamen met een groep II kappa lichte keten. kon er geen antigeen binding in ELISA gedetekteerd worden. Dezelfde substitutie in een anti-hCG antilichaam met een groep IV kappa lichte keten, en derhalve met leucine als wild type residu, had echter geen effekt op de antigeen bindings karakteristieken. In tegenstelling tot de boven beschreven $\mathrm{V}_{\mathrm{H}}$-mutatie, kon er hier wel een laag affiene binding met "surface plasmon resonantie" op een oppervlak met een hoge antigeendichtheid aangetoond worden. Een intermediaire trypsine resistentie kan een aanwijzing zijn voor een lokale verstoring van de struktuur, in plaats van een volkomen verkeerd gevouwen antilichaam domein. zoals dic werd waargenomen bij de hiervoor beschreven $\mathrm{V}_{\mathrm{H}}$-mutatie. Het lokale eflekt kan invloed hebben op de kanonieke struktuur van de dichtbij gelegen Ll-loop, en als gevolg hiervan, op de bindingsaffiniteit.

Gedurende de beide studies was het belangrijk om de hoeveelheid antigeen bindend scFv in periplasmatische extrakten, dat wil zeggen in ongezuiverde vorm, te bepalen. Hoofdstuk 3 beschrijft een methode, waarbij de concentratie van antigeen bindend scFv werd gemeten onder massa diffusie gelimiteerde omstandigheden met "surface plasmon resonantie" op het BlAcore systeem. Wanneer scFv geïnjekteerd werd op een oppervlak met hoge antigeen dichtheid, werd er een lineair verband verkregen tussen de bindingssnelheid en de concentratic aktief bindend antilichaam fragment. Uit een eenmalig gemaakte ijklijn, verkregen met gezuiverd $s c F v$, $k o n$ de hoeveelheid antigeen bindend antilichaam fragment in een monster snel bepaald worden aan de hand van de bijbehorende bindingssnelheid, die verkregen kan worden door injektie van dit monster op hetzelfde oppervlak.

Voor het vaststellen van zwangerschap worden verhoogde concentraties van het glycoproteine hormoon human Chorionic Gonadotropin (hCG) in urine gemeten met immunologische testen. De aanwezigheid van het nauw verwante human Luteinizing Hormoon $(\mathrm{hLH})$ en van vrije subunits of fragmenten afgeleid van de $\beta$-keten van hCG compliceren de metingen. Daarom wordt een kombinatie van monoklonale antilichamen gebruikt, die alleen het intakte hCG detekteert. In het bijzonder de kruisreaktiviteit met hLH vormt een groot probleem, omdat zowel hCG en hLH een identieke $\alpha$-keten bezitten, terwijl hun $\beta$-ketens over de eerste 114 aminozuren $85 \%$ homoloog zijn. In dit verband pasten wij faag display toe, met het doel hCG specifieke scFv-s te selekteren. In Hoofdstuk 4 wordt het gebruik van immuun banken beschreven, die gemaakt zijn met de milten van twee geïmmuniseerde muizen, voor de selektie van verschillende anti-hCG specifieke scFv-s. Uit de analyse met "surface plasmon resonantie" en Westem blot met de verschillende rekombinante antilichamen voor detektie, konden we een 
groep anti- $\beta$-core fragment, hCG specifieke antilichamen identificeren, als ook een een antilichaam, dat alleen de bioaktieve molekulaire vorm, namelijk de intakte dimeer, van hCG herkent.

ScFv-s met andere specificiteiten werden eveneens geselekteerd, zoals anti- $\alpha$-subunit specifieke antilichamen, en een groep van anti- $\beta$-core, hLH kruisreaktieve fragmenten. Vergelijking van de scFv-s geïsoleerd uit de immuunbanken met monoklonale antilichamen, die hun toepassing vinden in commercieel verkrijgbare zwangerschapstesten, toonde aan dat beide type antilichamen vergelijkbare affiniteiten hebben. Derhalve kan eenzelfde gevoeligheid verwacht worden van een assay, die de rekombinante antilichaam fragmenten zal bevatten.

\subsection{Anti-HIV-I SCFv-s}

De aanwezigheid van het Human Immunodeficiency Virus Type-1 (HIV-1) in humane sera wordt meestal vastgesteld aan de hand van het capside eiwit p24. Het strukturele eiwit is overmatig aanwezig in het capside, die het virale genoom hevat. Door screening met p24 antigeen tetsten is het mogelijk in een vroeg stadium sera van geïnfekteerde individuën te identificeren: met heden ten dage gebruikte testen kan de aanwezigheid van het p24 antigeen één tot twee weken eerder aangetoond worden dan een antilichaam respons tegen het virus. Het testen op p24 is eveneens van belang gedurende de behandeling van patiënten met anti-virale medicijnen. De spontane evolutie van medicijn-resistente varianten lijdt tot een reaktivatie van het virus. Om het virus in een vroege fase van de reaktivatie fase te detekteren, maar ook gedurende de primaire infektie, moeten de in de test toegepaste monoklonale antilichamen een hoge affiniteit bezitten, omdat deze eigenschap direkt gecorreleerd is met de gevoeligheid van de test. Ten gevolge van de heterogeniteit van de verschillende HIV-stammen, is het tevens van belang dat de antilichamen geconserveerde epitopen van p24 herkennen.

De selektie van een aantal anti-p24 scFv-s uit een immuun bank staat weergegeven in Hoofdstuk 5. Na immunisatie van een muis met een viraal lysaat van HIV-1-virus, werd RNA geïsoleerd uit de milt en vervolgens gebruikt voor de konstruktie van het repertoire. Eén van de geselekteerde antilichamen herkent een immuundominant epitoop van p24, zoals uit de pepscan analyse geconcludeerd kon worden. Een voordien beschreven hybridoma geproduceerd monoklonaal antilichaam met dezelfde specificiteit, gebruikte de identieke kombinatie van $\mathrm{V}_{\mathrm{H}^{-}}$ en $V_{K}$-regio's. Vandaar dat deze scFv én van de zeldzame voorbeelden is, waarbij de originele paring in een uit een immuun bank geïsoleerde antilichaam fragment terug gevonden wordt. De affiniteiten van de geselekteerde fragmenten waren vergelijkbaar met die van monoklonale antilichamen, die toegepast worden in een commercieel verkrijgbare p24 antigeen test.

In plaats van het immuniseren van muizen, is er een humane immuunbank gebruikt voor de selektie van antilichaam fragmenten gericht tegen HIV-I envelop afgeleide eiwit-antigenen. Hoofdstuk 6 behandelt de konstruktie van de scFv bank van slechts een klein aantal perifere bloed lymfocyten, die gedoneerd waren door een seropositief individu. Een belangrijk argument 
om humane antilichamen te selekteren is het potentièle verschil in antigeen presentatie gedurende immunisatie van een muis, in vergelijking met de virus replikatie in de mens. Eveneens kunnen, door toepassing van de humane antilichaam fragmenten in ELISA voor capture en/of detektie, vals-positieve ELISA signalen vermeden worden, die het gevolg zijn van sporadisch voorkomende sera met humaan anti-muis antilichamen (HAMA).

Biopanning met het envelop precursor eiwit gp160 resulteerde in de selektie van twee families van scFv-s. Eén groep herkende een conformationele epitoop van het rijpe envelop eiwit gp120, de andere familie was specifiek gericht tegen het transmembraan glycoproteine gp4l; beide strukturele eiwitten worden door proteolyse uit gp 160 gevormd. Aan de hand van een sequentie homologie met eerder beschreven Fab-s, die eveneens geïsoleerd waren uit een immuun bank, kon geconcludeerd worden dat onze scFv-s reageren met de kluster III-epitoop van gp41.

Competitie ELISA toonde aan dat in het bijzonder de epitoop van de de anti-gp41 scFv-s herkend wordt door een groot panel van sera van Noord-Amerikaanse en Afrikitanse seropositieven. Ten gevolge van het sterk geconserveerde karakter van diens epitoop, lijkt de anti-gp41 scFv de meest geschikte kandidaat voor toepassing in cen in vitro diagnostische test.

\subsection{De konstruktie van en de selektie uit een nä̈eve humane bank}

Het tamelijk tijd-verslindende immuniseren en het herhaaldelijk maken van immuun banken, die nodig waren voor het selekteren van de anti-hCG- en anti-HIV-1-antilichaam fragmenten, die beschreven zijn in de voorgaande hoofdstukken, kan in het geheel vervangen worden door toepassing van een naïef repertoire. Uit publikaties blijkt dat afhankelijk van de grootte van de bank hoog affiene antilichamen tegen ieder antigeen verkregen kunnen worden uit dergelijke bamken.

Hoofdstuk 7 behandelt de konstruktie van een zeer grote naïeve Fab bank, die 37 miljard faag klonen bevat. De kwaliteit werd geanalyseerd door de selektie met twee eiwit antigenen. welke zijn tetanus toxoid en het tumor geassocieerde antigeen mucin-1, en het hapteen phOx, en de daaropvolgende affiniteitsbepalingen van de geselekteerde Fab-s. Als maat voor de affiniteit werd de snelheid van dissociatie van het antigeen-antilichaam complex bepaald met "surface plasmon resonantie" door de injektie van periplasmatische frakties; de dissociaties verliepen met een monofasische kinetiek ten gevolge van het pure monovalente karakter van de Fab-s.

De echte uitdaging was de selektie van de naïeve bank met de glycoproteine hormoon afgeleiden, het thema, wat als een rode draad door dit proefschrift (Hoofdstuk 1 tot en met 4) loopt. Selektie met human Chorionic Gonadotropin (hCG) leverde een groep Fab-s op, die de holo-vorm van het hormoon specifiek herkende, als ook $\beta$-keten specifieke Fab-s, die kruis reageerden met human Luteinizing Hormone (hLH). Bij gebruik van hLH voor selektie werden Fab-s gevonden, die alleen de dimeer van het gebruikte hormoon herkenden, evenals een groep antilichamen gericht tegen de $\alpha$-keten, en derhalve kruisreaktief tegen de andere leden van de 
familie van glycoproteïne hormonen. Toepassing van human Follicle Stimulating Hormone $(\mathrm{hFSH})$ resulteerde in de selektie van $\beta$-keten, hormoon specifieke antilichamen en eveneens van $\alpha$-keten, hL.H/hCG kruisreaktieve Fab-s. Van alle hCG-reaktieve antilichaam fragmenten werd, na zuivering met antigeen kolom, de kinetiek van antigeen binding gemeten met "surface plasmon resonantie". De affiniteit lag tussen de 2.7 en $38 \mathrm{nM}$, wat vergelijkbaar is met die van antilichamen verkregen uit een immuun bank of die geproduceerd worden door hybridoma cellijnen. 


\section{EPILOGUE: DISCUSSION}




\section{Diagnostic value of the recombinant antibody fragments and their engineering; general conclusions}

The technology of phage antibody library display was developed in the beginning of the 90s, and at present is well accepted in the academic environment. Yet, this is one of the first extensive studies to date in which phage antibodies to a series of main diagnostic targets have been isolated, and the utility of the technique is shown outside the research laboratory environment. This is caused by the combination of the experimental difficulty of the procedure and the scepticism and resistance of more traditional immunologist to this change in technology.

As was shown in the studied cases of this thesis, we can state that the phage antibody repertoire technology yields antibody fragments with similar specificities and affinities as found with hybridoma technology. This can go as far as isolating the nearly identical Variable gene sequences, as was found in a single case: a phage-library selected anti-HIV p24 phage scFv antibody fragment contained identical $V_{H}$ and $V_{L}$ gene sequence as those found in a previously described monoclonal antibody. But mostly antibodies of slightly different genetic make-up were identified, but with a speed and ease that compares favourably with the hybridoma technology. For example, in the case of the hCG antigen, the spectrum of fine specificity found in phage repertoire derived antibodies matched perfectly that of hybridoma-derived antibodies. For the construction of the phage repertoires, only two mice were immunised with hCG, whereas thirty two (labour intensive) fusions were needed for the generation of the different monoclonal antibodies via hybridoma technology. Besides the time advantage for the phage approach, two other major bonuses for the technology come from the possibility to prepare unlimited quantities of the selected poly- or monoclonal phage (from the stored bacterial stocks), and to vary at will the in vitro selection method, enabling the isolation of infrequent or rare variants. To illustrate this last point, we cite the difference that we encountered when using biopanning on immobilised p24 versus selecting with biotinylated p24. In the latter case many different antibody variants were selected versus only one single type of scFv for the panning procedure. Similarly, in solution selection using hCG gave the highest degree of variation in respect to the specificity, while with coated hCG antigen only $\beta$-core specific scFv's were found. The use of soluble antigen enables the selection on affinity in a controllable manner, because the applied antigen concentration functions as a threshold. Unlike hybridoma derived antibodies, selected phage antibodics come directly with their clonal genetic message. Therefore rapid identification of the DNA type or fingerprint of each individual selected phage antibody may serve as additonal test or complementary assay to test the diversity of a selected population. This is illustrated by an example from the anti-hCG scFv story: at the time of screening of the selected scFv's, material of the related antigens hLH and hSFH were not available yet, but the different types of antibodies were discriminated readily by their fingerprints. Analysis of their specificity with the related hormones confirmed the classification previously made by fingerprint analysis. Thus, 
when comparing with the hybridoma technology, the cloning of phage antibodies from the repertoires made from the spleen cells of immunised animals is generating antibodies more rapidly, the antibodies are easier to analyse and the spectra of fine specificities and genetie composition of the selected phage antibodies are at least as broad if not more diverse. We foresee that the use of hybridomas, for scientific, technical, ethical as well as financial reasons, will eventually be surpassed by the use of an in vitro selection procedure. However, nearly a decade after the initial publication on the concept of the use of in vitro libraries by Milstein and Winter to by-pass the animal-made antibodies, it can not be ignored that $95 \%$ of the reserach laboratories still use the hybridoma technology. We contribute this to the difficulties in establishing the phage technique in a non-molecular biological laboratory, and also to the timeconsuming constructing of the phage antibody library every time after immunisation. The immunisation route requires the antigen to be non-toxic and immunogenic to the host, and is time consuming: immunization costs 4 to 12 weeks at least; the construction of an immune repertoire takes at least another 4 to 6 labour-intensive weeks. Most notable achievements in the immune library field have addressed the characterization of the human immune response in disease, using patient derived immune repertoires for the selection, as exemplified in this thesis by our human scFv-s directed against the HIV-l envelope proteins. Due to its speed and ease over more conventional techniques to study natural humoral responses, this will be an area which will be used more extensively in the future, despite the loss of original combination of the antibody's variable regions in the cloning procedure.

The true revolution, however, must come from the use of non-immune library sources. The repeated immunization and construction of libraries may be avoided by the use of a large antibody repertoire, from which mediate to high affinity antibodies against any type of antigen are retrieved, as exemplified by the description in this thesis of the large 'naive' phage Fab library. Indeed, the selection from such a naive repertoire permitted the direct generation and identification of monoclonal antibody fragments in less than a month. Most impressive were the results obtained when the large naive Fab library was used for selection with glycoprotein hormone hCG and relatives. Antibodies recognising hCG only were obtained without application of special selection protocols; even anti-hLH specific Fab's were selected, a species which is extremely difficult to isolate using hybridoma technology. Due the use of a very large phage library ( 37 billion clones), the affinities of the recombinant Fabs were comparable to affinities of antibodies made by hybridoma technology or selected from an immune repertoire. All antibodies were isolated by one scientist in approximately three months, including the complete characterisation on fine specificity, affinity and sequence. For comparison, this is the minimal time needed for immunisations only. Already, several companies have initiated phage antibody selection services to customers wishing to rapidly gain access to phage antibodies, and with the increasing spread of these large naive phage libraries in the scientific community, the use of phage antibodies is bound to by-pass animal use for antibody generation in the future. With the 
apparent scope of automating the in vitro selection procedures, we anticipate that the isolation of antibodies to defined antigens in the future will be a matter of days rather then months. It is without doubt that this will be of impact in all fields of science in which antibodies are used as probes, reagents or therapeutics.

Besides the possibilities offered by the in vitro antibody generation and selection procedures, the genetic engineering of the available antibody genes forms a challenging opportunity for the future. Both engineering for higher expression, for improved affinity and for provison of a novel effector function may be envisaged. For example, by in vitro mutagenesis of the antibody sequence, we identified a key residue in the certain murine $V_{H}$ genes which are extremely important for folding. Knowledge of the crucial residues in protein folding and stability, will eventually be useful to increase production levels and thus reduce costs with which antibody-based products may be made. Further, it may aid in building qualitatively better phage libraries. Mutagenesis of residues localised in the CDRs or at random within the antibody variable regions, in combination with sensitive selection methods permitting discrimination hetween low and high affinity antibodies, may enable the generation of higher affinity antibody variants. Several examples of picomolar affinity phage antibodies have already been described in reports from the States. The in vitro selection procedure appears less restricted than the immune system itself by the affinity that can actively be selected for.

Phage antibodies are bare antigen-binding modules, and come without effectors. There is now, however, a very large list of proteins or peptides the antibody may be genetically fused to, to provide a purification tag, an effector or a detection tag. Aiming at the application in diagnostics, the opportunities of genetic engineering were demonstrated elegantly in our laboratory (P. Oudshoom, B. van Lith and P. Hiemstra unpublished data) and more recently by others. By fusion of the enzyme alkaline posphatase from $E$. coli to the carboxyterminus of one of the anti-hCG scFv-s, an in vivo produced antibody conjugate was obtained, which could be used in immunoassays without further purification. As another example, genetic fusion to a peptide sequence that was in vivo biotinylated in $E$. coli, yielded an antibody fragment which could be captured as a functional molecule on a streptavidin coated surface. The biotinylated scFv also gained avidity and thereby affinity by binding to soluble streptavidin. Finally, fusion of the His ${ }_{6}$-tag facilitated coating of scFv to a polystyrene surface, without loosing its capacity to bind antigen. Such donwstream phage antibody engineering is necessary to provide the crucial detection or effector mechanism for use of the antibody.

In conclusion it can be stated that both the application of repertoire technology and the possible engineering techniques are extremely valuable for the generation of diagnostic reagents. The technology might enhance the development of a completely new generation of diagnostic products, especially when the skills and efforts of experts in both types of disciplines, i.e., genetic engineering and assay development, will be combined. 


\section{Dankwoord}

Zoiets waanzinnigs doe je niet alleen, vandaar dit uitgebreide dankwoord. Mijn dankwoord kan niet anders beginnen, dan deze aan de persoon te richten, die voor mij persoonlijk de meest waardevolle bijdrage heeft geleverd, Bert Kazemier. Het plezier waarmee we de eerste gelukte selekties beleefden, welke uiteindelijk positieve ELISA-signalen opleverden, zal me altijd bij blijven. Bert, nogmaals hartstikke bedankt voor de geweldige tijd die ik met je heb mogen beleven, voor het perfekte werk dat uitgevoerd is en voor het plezier dat we hebben gehad.

Onmisbaar is natuurlijk de bijdrage van mijn co-promotoren Bob van Gemen en Hennie Hoogenboom geweest. Bob, je steun tijdens mijn promotie heb ik enorm gewaardeerd. Hennie, bedankt voor je deskundige begeleiding. Jan-Willem als promotor, voor het aanvaarden van de moeilijke taak om een promotie-onderzoek in de Molekulaire Immunologie te begeleiden.

Verder wil ik Piet Boender bedanken, onder wiens leiding in de groep Molekulaire Biologic zeer plezierig gewerkt werd en met wie ik al vele jaren samen gewerkt had. Mijn afsicheid van Organon Teknika voelde ik als een scheiding van mijn eeuwige vriend Piet, met wie ik zovecl hoogte als ook dieptepunten gedeeld had. Piet, heel erg bedankt, vooral voor je bijdrage aan de intellektuele zijde van het proefschrift, jij bracht me altijd de meest recente en interessante papers, hielp me bij het opstarten van het promotie onderzoek, en was altijd goed voor een prettig gesprek bij de koffie of de lunch.

Bart, Piet Hiem, Dick, Liesbeth, Femke en Moniek als direkte kollega's, en Peter bovendien nog als fietsmaatje, heel erg bedankt voor de fijne tijd. Arie, als modeller verantwoordelijk voor de mooiste plaatjes van "onze" antilichamen. is een echte vriend geworden, en tevens medeauteur op een tweetal papers. Veel dank gat eveneens uit naar de andere BRU-genoten, betrokken bij aanleveren van monoklonalen. ELISA-platen, en nog veel meer andere materialen en ideeën. Mark van Roosmalen moet ik er uitlichten, aan hem heb ik mijn up-to-date kennis van computers en programmatuur te danken. Het beschreven hCG werk zou niet mogelijk zijn geweest zonder mijn steun en toeverlaat Bert van der Weijer, hetzelfde geldt voor het HIV-werk voor een kollega van het eerste uur uit de Organontijd Tonnie Jacobs. Bij het onderwerp HIV moeten mijn adviseurs Jan-Alber Hellings en Jan van Binsbergen in een adem genoemd en bedankt worden.

Dan is er nog het arsenaal aan studenten en stagiaires, die hebben meegeholpen. Dit betreft Richard Peters, Ine van Kuijk, Yvette de Hingh, en Andrew Bakker, die via de afdeling Molekulaire Biologie van de Katholieke Universiteit Nijmegen bij ons terecht kwamen. En verder de HLO-stagiaires Nancy van Daal, Sunita Jaghjhoorsingh, en Cora de Jongh.

Mijn oud-kollega's van Organon, Peter van Weezenbeek, Rob Nelissen, Cees Meeuwisse en Paul Vink, voor het ter beschikking stellen van de display vektor pVI en de vele humane primers, alsmede voor de nuttige diskussies over het onderzock. 
Uit mijn Maastrichtse tijd wil ik op de eerste plaats mijn kamergenoot Roel Kuijer bedanken voor de prettige atmosfeer. Verder alle CESAME leden en andere collega's van de vakgroep Pathologie, en dan met name Anneke, Nicole en Simon voor hun aktieve rol bij het maken van "de bank". En tenslotte Han, voor de door-de-weekse gezelligheid en huisvesting.

Tenslotte mijn huidige collega's van Unilever, voor het geduld dat ze betrachten voor het te laat inleveren van mijn SOP-jes en pUR-plasmides. Met name Marcel, op wiens verzoek ik mijn persoonlijke dankwoord van drie A4-tjes gericht aan hem terug gebracht heb tot én regel, voor de vele nuttige tips bij het maken van dit boekwerk.

Dan nog mijn moeder, die het einde van de promotietijd niet meer mee heeft mogen maken, maar apetrots zou zijn geweest.

En tenslotte Willeke, voor de mentale steun en het geduld dat je had tijdens mijn lange werkdagen. En natuurlijk Monique, Saskia en Eline, voor de noodzakelijke afleiding tijdens het schrijven. 


\section{Curriculum vitae}

Hans de Haard werd op 11 juli 1959 geboren te Beek bij Ubbergen. In 1977 behaalde hij het eindexamen HAVO, en, na één jaar HTS werktuigbouwkunde in s' Hertogenbosch gedaan te hebben, werd begonnen met de opleiding HBO-B biochemie te Oss. Na het behalen van het diploma in 1981, werd de avondstudie HTS richting chemie in 1983 met succes afgesloten.

Van 1982 tot begin 1984 was de auteur werkzaam bij de afdeling Hepatologie van de Katholieke Universiteit Nijmegen, waar hij onder leiding van Prof. dr. Sing Hiem Yap meehielp aan de promotie van dr. Hans Princen en dr. Han Moshage. In de periode 1984 tot eind 1985 werd de scholing in en de interesse voor de Molekulaire Biologie opgedaan bij de afdeling Molekulaire Biologie van het Nederlands Instituut voor Zuivelonderzoek te Ede onder de bezielende leiding van Prof. dr. Willem de Vos. Vervolgens kreeg hij een betrekking bij het diagnostika bedrijf Organon Teknika (onderdeel van AKZO-NOBEL), alwaar hij onder leiding van dr. Piet Boender binnen de unit DRL-E onderzoek verrichtte aan het hepatitis nonA-nonBvirus.

Na de verhuizing van Organon Teknika in 1989 natar Boxtel was de auteur als projektleider binnen de groep Molekulaire Biologie van de Biosciences Research Unit (BRU) verantwoordelijk voor de klonering en expressie van antigenen in $E$. coli.

In 1991 startte het promotie onderzoek, gedeeltelijk gefinanceerd met een PBTS-subsidie, die door dr. Chris van Eekelen (als Unit-Manager van de BRU) en dr. Peter van Weezenbeek (Projektleider binnen de toenmalige Biotechnology Group van Organon) aangevraagd was, en dat uitgevoerd is bij Organon Teknika.

Vanaf begin 1997 werkte hij in de groep CESAME van de vakgroep Pathologie aan de Universiteit Maastricht.

Sinds januari 1998 is de auteur als manager werkzaam binnen de unit Functional Biomolecules van de groep Biotechnology van Unilever Research Laboratorium Vlaardingen.

Hij is getrouwd met Willeke Hoekman, en vader van Monique, Saskia en Eline. 
